\title{
CELL MOTILITY IN MICROFLUIDIC ENVIRONMENTS
}

\section{DISSERTATION}

for the award of the degree

"Doctor rerum naturalium"

Division of Mathematics and Natural Sciences

of the Georg-August-Universität Göttingen

submitted by

ERIC STELLAMANNS

from Adendorf 
Member of the Thesis Committee:

Prof. Dr. Thomas Pfohl, Reviewer

Member of the Thesis Committee:

Prof. Dr. Jörg Enderlein, Co-reviewer

Member of the Thesis Committee:

Prof. Dr. Marc Timme, Co-reviewer 
It is declared that the presented thesis has been written independently and with no other sources and aid than quoted.

Eric Stellamanns 
meinen Eltern 
Trypanosomes are single-celled bloodstream parasites and causative agents of African Sleeping Sickness in humans and Nagana disease in domestic livestock. The pathogen is transmitted by the bite of the tsetse fly and lethal to the infected host if left untreated. Eponymous for the Trypanosoma genus name (Trypanosoma: "drilling body") is the striking nature of their movement which is often described by the spiral motion of a corkscrew. However, looking at trypanosomes at high spatiotemporal resolution, we find that the way the cells move is more complex than described before and changes over time and cell. Apart from the question how trypanosomes move, we find ourselves confronted with the question why trypanosomes move at all? In this context, M. Engstler et al. have shown that active movement is essential for cells that are exposed to hostile antibodies. Hydrodynamic flow induced by active movement of the cell leads to a delocalization of antibodies that have bound to the cell surface: Antibodies exposed to the flow around a forward swimming cell are driven backwards into the flagellar pocket where they are taken up by endocytosis and rendered harmless by subsequent digestion. In contrary, a backward swimming cell is accumulating antibodies at the tip of the flagellum and gets digested itself by the host's immune system. If the described mechanism of hydrodynamic protein sorting is a ubiquitous feature in nature, it has to be proven in more detailed studies of cell motility as well as the involved hydrodynamic condition.

The aim of this thesis is to study and quantify the movement of trypanosomes in their microfluidic environments in order to help understanding the mechanisms and reasons of their motility. To achieve this goal we constructed an optical trapping fluorescence microscope optimized for high spatiotemporal resolution and reduced phototoxicity. In combination with advanced microfluidic methods we were not only able to control hydrodynamic flow conditions and spatial confinement, but also to position, manipulate and measure forces on the single cell level, as well as to specifically label single living cells in the microflow.

In this work we could show for the first time that using strongly focussed diode lasers it is possible to optically trap living trypanosomes over time scales of $t_{\text {halflife }} \leq 15 \mathrm{~min}$, without inducing significant photodamage. The optical stall forces acting on trypanosomes were determined and used to measure the propagation forces of single and dividing trypanosomes. In combination with automated image processing routines we also analyzed the positioning of trypanosomes within the optical trap and found distinct trapping loci which could be correlated to structural features of trypanosomes. 
With the described trapping characteristics of trypanosomes, we analysed their motility in optical confinements and were able to distinguish running cells from tumbling ones. Moreover, several features specific to our setup allowed for the analysis of long time (5 min) dynamics with high temporal resolution (100 fps). We could not only link tumbling and running motility to certain flagellar beating patterns, but also show how these patterns change over time. The motility patterns could be quantitatively described in terms of frequency, direction and velocity.

Introducing a steerable second optical trap into the system, we measured the flagellar beating force of a trypanosome's anterior tip acting on a polystyrene bead that was trapped in close proximity.

With a second trap at hand, we could examine hydrodynamic interactions between two trapped rotating trypanosomes. By successive reduction of the trapping distance of two living trypanosomes, we saw increasing correlation between the flagellar beating of both cells, finally resulting in cell synchronisation at distances $d \approx 6 \mu \mathrm{m}$. Together, these findings strongly support comprehensive research toward understanding not only trypanosome motility but also hydrodynamic synchronisation of complex swimmers at low Reynolds numbers.

As shown in this thesis, the combination of microfluidics and optical trapping enable well controlled, detailed studies of trypanosome propulsion, force propagation, and synchronisation and provide a toolbox for future biophysical investigations at the single cell level. 
Trypanosomen sind einzellige Blutstromparasiten und Erreger der in Afrika weit verbreiteten Schlafkrankheit des Menschen, sowie der Nagana Seuche in landwirtschaftlichen Nutztieren. Die Krankheit wird durch den Biss der Tsetsefliege übertragen und endet ohne medizinische Behandlung mit dem Tod des jeweiligen Wirtes.

Der Name ist der griechischen Sprache entlehnt (Trypanosoma $=$ Bohrkörperchen) und beschreibt bildlich den unter dem Mikroskop sichtbaren Bewegungsablauf, welcher auf dem ersten Blick dem Verlauf einer Korkenzieher Spindel ähnelt. Bei genauerer Betrachtung wird jedoch deutlich, dass die tatsächliche Bewegung der Zellen nicht nur wesentlich komplexer als bisher beschrieben ist, sondern auch von Zelle zu Zelle sowie über die Zeit variiert. Des Weiteren gilt die Frage zu klären, warum sich Zellen, die im Blutstrom leben, überhaupt aktiv bewegen, sind Sie doch nicht annähernd schnell genug, um gegen den Strom zu schwimmen. In diesem Kontext konnten Prof. M. Engstler und Kollegen zeigen, dass nur solche Zellen im Blut des Wirtes überleben, die sich aktiv bewegen. Sie machen hierfür den hydrodynamischen Fluss verantwortlich, welcher durch die aktiven Schwimmbewegungen der Zelle erzeugt wird: Antikörper, welche an der Oberfläche von Trypanosomen gebunden haben, werden mit Fluss und somit entgegen der Schwimmrichtung zum „Kopfende“ der Zellen getragen, wo diese aufgenommen und somit unschädlich gemacht werden können. Rückwärts schwimmende Mutanten hingegen, häufen diese Antikörper am „Fußende“ der Zelle an, dort können diese nicht abgebaut werden und bewirken somit ihrer Aufgabe entsprechend den Abbau der Trypanosomen selbst. Um herauszufinden, ob der beschriebene Mechanismus hydrodynamisch getriebener Proteinsortierung ein einzigartiges Phänomen oder aber ein in der uns bekannten Natur weit verbreitetes Prinzip darstellt, müssen wir a priori den genauen Bewegungsablauf sowie dessen hydrodynamische Wirkung auf die Zellen verstehen.

Das Ziel dieser Arbeit ist den Bewegungsablauf von Trypanosomen in ihrer mikrofluidischen Umgebung messbar zu machen und zu beschreiben, um auf diesem Wege zu klären, warum sich Trypanosomen auf welche Art und Weise bewegen. Um dieses Ziel zu erreichen, haben wir zunächst ein sehr lichtempfindliches Fluoreszenzmikroskop zur optischen Mikromanipulation lebender Zellen entwickelt, welches bei hoher raumzeitlicher Auflösung und hoher optischer Kraftwirkung minimale phototoxische Wirkung zeigt. In Kombination mit speziell angepassten mikrofluidischen Methoden, erlaubt dieses Instrument die Manipulation einzelner lebender Zellen, sowie die präzise Kontrolle über Strömungsbedingungen und die räumliche Umgebung im Umfeld der Zellen. Dieser Aufbau ermöglicht also nicht nur einzelne Zellen gezielt im dreidimensionalen Raum zu positionieren, sie $\mathrm{zu}$ bewegen und deren Kräfte $\mathrm{zu}$ messen, sondern auch das gezielte 
Markieren lebender Zellen mit Fluoreszenzmarkern im Mikrofluss, sowie die unmittelbare Beobachtung und Quantifizierung des gesamten Prozesses.

In dieser Arbeit konnte zum ersten Mal gezeigt werden, dass es möglich ist, lebende Trypanosomen über einen Zeitraum von $t_{\text {halflife }} \leq 15$ min mit Hilfe eines stark fokussierten Diodenlaser festzuhalten, ohne die Zellen dabei erheblich zu schädigen. Die dabei ausgeübten optischen Haltekräfte wurden bestimmt, um die Fortbewegungskräfte einzelner sowie in Teilung befindlicher Zellen zu messen. Gleichzeitig war es möglich mit Hilfe von automatisierten Bildbearbeitungsroutinen quantitativ den Ort der höchsten optischen Kraftwirkung zu bestimmen und diesen in Verbindung mit bekannten Zell Strukturen zu setzen.

Nach erfolgreicher Charakterisierung der optischen Kraftwirkung auf die Zellen, wurde die Bewegung der Zellen also solche innerhalb des optischen Potentials analysiert. Dabei konnten sogenannte „Läufer“ von „Torklern“ getrennt und die maßgeblichen Unterschiede im jeweiligen Bewegungsablauf zeitlich hoch aufgelöst (100fps) und gleichzeitig über lange Zeiträume (5min) quantifiziert werden. Es war möglich, diese Bewegungsarten auf bestimmte Schlagmuster des Flagellums zurückzuführen und zu zeigen, dass sich diese über die Zeit ändern können. Durch den Einbau einer zweiten frei beweglichen optischen Falle war es weiterhin möglich, die Schlagkräfte zu messen, welche ein festgehaltenes Trypanosom mit Hilfe seines Flagellums auf Objekte in Reichweite ausüben kann. Durch diese Erweiterung konnten nun auch zwei Zellen gleichzeitig festgehalten und manipuliert werden, so dass untersucht werden konnte, ob die Zellen sich gegenseitig hydrodynamisch beeinflussen. Dabei hat es sich gezeigt, dass sich die Zellen bei Abständen von D > $6 \mu \mathrm{m}$ in Ihrem Bewegungsablauf nicht beeinflussen, bei kleineren Abständen jedoch eine Synchronisation der Zellbewegung zu beobachten ist.

Zusammengefasst unterstreichen diese Ergebnisse das Potential der angewendeten Methodik, sowie die Wichtigkeit dieser Forschung für das Verständnis der Zellbewegung, nicht nur am Beispiel von Trypanosoma brucei brucei, sondern auch für Effekte wie hydrodynamische Synchronisation komplexer Bewegungsabläufe von Organismen im Bereich kleiner Reynolds Zahlen. Wie in dieser Arbeit gezeigt werden konnte, ermöglicht die Kombination aus optischer Mikromanipulation und mikrofluidischer Methodik präzise Kraftmessungen an lebenden Zellen unter genau kontrollierten Strömungsbedingungen und birgt ein großes Potential für zukünftige Forschung im vielen Bereichen der Biophysik. 


\section{Abstract}

1. INTRODUCTION 1-8

$\underline{\text { THE SLEEPING SICKNESS }}$

TRYPANOSOMA BRUCEI BRUCEI

2. METHODS

2.1. MICROFLUIDICS

$\underline{\text { MiCROFLUIDICS }}$

$\underline{\text { PHOTOLITHOGRAPHY }}$

$\underline{\text { PDMS MOLDING }}$

PRECISION OF METHOD

MiCROFLOW

$\underline{\text { STOPFLOW }}$

2.2. OPTICAL TRAPPING

$\underline{\text { THEORY OF OPTICAL TRAPPING }}$

OPTICAL TRAP SETUP

$\underline{\text { MICROSCOPE SETUP }}$

CALIBRATION OF THE OPTICAL TRAP

2.3. IMAGING

$\underline{\text { IMAGE PROCESSING }}$

$\underline{\text { PROCEDURES }}$

2.4. Cell biology 
3. OPTICAL TRAPPING OF TRYPANOSOMES

OPTICAL TRAPPING OF BIOLOGICAL OBJECTS

OPTICAL TRAPPING OF TRYPANOSOMES

OPTICAL STALL FORCES ON TRYPANOSOMES

TRYPANOSOME CELL SHAPE

ORIENTATION IN FLOW

REFRACTIVE INDEX OF TRYPANOSOMES

TRAPPING LOCI OF TRYPANOSOMES

$\underline{\text { TRYPANOSOME FORCES }}$

$\underline{\text { PHOTOTOXICITY OF OPTICAL TRAPPING }}$

CHAPTER SUMMARY AND CONCLUSIONS

4. Motility OF TRYPANOSOMES

$\underline{\text { PHYSICAL PRINCIPKLES OF CELL MOTILITY }}$

$\underline{\text { TRYPANOSOME MOTILITY IN LITERATURE }}$

TRYPANOSOME MOTILITY IN CONFINEMENT

TRYPANOSOME MOTILITY IN OPTICAL CONFINEMENT 


\section{THE SLEEPING SICKNESS}

\section{INTRODUCTION}

Sleeping sickness is common language for the human African trypanosomiasis disease: HAT. As the name implies, the sickness is caused by trypanosomes and spread by the bite of the tsetse fly throughout most countries of Africa. Trypanosomes are bloodstream parasites that develop inside an arthropod vector and multiply within their hosts that can both be human or animal.

Trypanosoma brucei brucei were first described by David Bruce in the late $19^{\text {th }}$ century to be the pathogen for Nagana in cattle (animal African trypanosomiasis disease, AAT) that suffer from systemic inflammations and profound lethargy just before death (1). Several decades later the human pathogen for HAT was discovered to be of the same species and named by its local origin Trypanosoma brucei gambiense and Trypanosoma brucei rhodesiense. Currently there are 60 million people at risk of HAT in 36 sub-Saharan countries of Africa, while simultaneously AAT prohibits entire populations from animal husbandry (2).

Fig.1.1: GLOBAL STATUS HAT: (WHO 2004)

- Cases: 300’000-500’000 annually

- Deaths: 66'000 annually

- Disease burden: 2.05 million Disability Adjusted Life Years (DALY)

- People disabled: 100’000

- Prevalence: $>70-80 \%$ in villages in certain foci

\section{Clinical PRESENTATION}

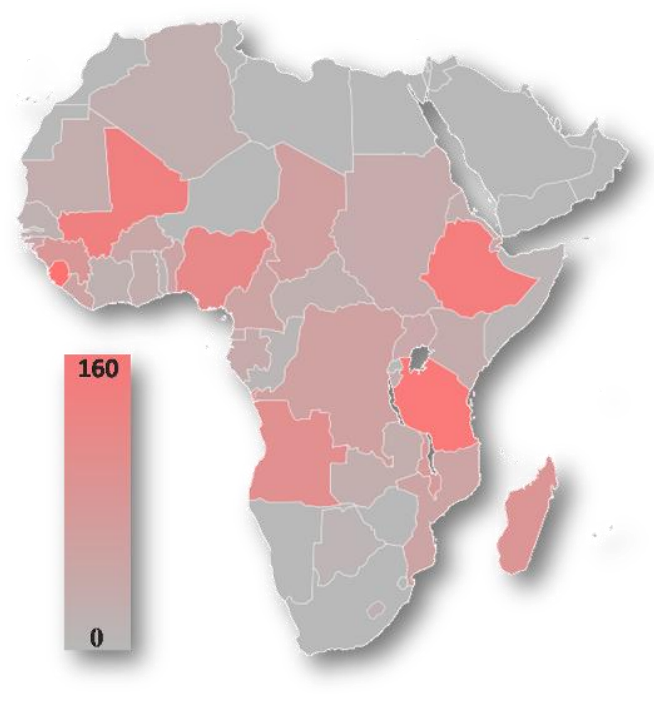

Deaths per 100.000 population

Cattle infected by trypanosomes are lost to milk as well as meat production, because of chronic illnesses and eventually die from secondary infections ( $\underline{3})$.

Human infections by T. . gambiense and T. b. rhodesiense are always fatal if left untreated, but epidemiology and symptoms vary. Infections by $T$. $b$. gambiense cause a chronic disease which can go unnoticed for several months or even years, whereas an infection by $T . b$. rhodesiense takes a faster course and symptoms already appear after a few weeks, followed by death within a year. The epidemiology is described in two stages: 
$1^{\text {st }}$ stage: After the bite of an infected tsetse fly, the parasites start to proliferate subcutaneously and cause a local swelling. As the disease progresses they spread through the lymphatic system into the bloodstream and cause a generalized infection, accompanied by waves of fever and severe organic malfunction in case of T.b. rhodesiense (3).

$2^{\text {nd }}$ stage: The second stage is marked by an invasion of the central nervous system, CNS, through the blood brain barrier, BBB, by a yet unknown mechanism. The symptoms give name to the sickness: strong headaches are followed by changes in mentality, cognition and motor functions to terminate in a somnolent state described as sleep ( $\underline{3})$.

\section{Treatment}

The diagnosis of HAT requires a high degree of expertise and training in pathogen detection as well as infectious stage determination. Frequent titre measurements are necessary, because of strong variations in pathogen concentrations. CNS infections can only be determined by lumbar puncture.

Despite the high burden of the disease, little progress has been made in drug development. Only four drugs are available as treatment for human African trypanosomiasis, Suramine (1921), Pentamidine (1941), Melarsoprol (1949) and Eflornithine (1990) (ㅁ). The treatment depends on the stage of infection, whereas Suramine and Pentamidine are considered of less toxicity, only Melarsoprol and Eflornithine can pass the blood brain barrier and reach the parasite in the second stage of infection.

Eflornithine is less toxic than Melarsoprol, but it is only active in T.b. gambiense, Melarsoprol however is an arsenic compound that shows severe side effects on the CNS of which 3-10\% are considered to be fatal (WHO 2010).

\section{Perspectives}

During epidemic periods reported in between 1986 to 2004, the number of actual infections was estimated with 300000 cases and reached almost 50\% in several disease foci. At these times, HAT was considered to be greatest cause of mortality, even ahead of HIV in certain areas of Africa.

In 2005, treatments were reinforced and supply of Melarsoprol as well as Eflornithine, produced by Sanofi-Aventis and Bayer healthcare, was reinsured with the help of the WHO and other public as well as private cooperation. 
The reestablishment of treatment lead to a decrease of reported cases below 10000 people in the year 2009 (WHO). Indeed, early detection and medical supply has proven to be very effective in HAT control, but elimination of the disease in the animal reservoir will not be possible (WHO 2010). For this reason new infections will not cease to appear, rendering continuous treatment indispensable.

Alternative drugs need to be developed that are less hazardous in both early and late stage infection treatment. To prevent from dangerous and expensive lumbar puncture, needed to detect trypanosomes that have already invaded the CNS (late stage infection), a combined treatment of both stages is necessary. Such a dual potent treatment, combined with moderate side effects, would allow for preventative medication by low trained personal. The same is true for human livestock, combined with a positive impact on the ecologic situation of the affected countries.

A better understanding of trypanosome ecology, pathology and cell biology is necessary to maintain control and decrease the burden of African trypanosomiasis.

\section{TRYPANOSOMA BRUCEI BRUCEI}

\section{PHYLOGENY}

Trypanosomes are unicellular eukaryotes of the protozoan kind (Fig. 1.2). They are members of the mastigophora group which describes all protozoa that move by flagella. They are divided from other flagellates by the presence of a kinetoplast. The pathogenic species of the sleeping sickness (HAT and AAT) consists of five subspecies:

1. T.b. brucei (infects cattle, camel, animal game (ㅁ))

2. T.b. gambiense (infects human, pig, sheep (ㅁ))

3. T.b. rhodesiense (infects cattle, human (ㅁ))

4. T.b. equiperdum (infects horses, mules (ㄱ))

5. T.b. evansi (infects horses, camel (ㅁ))

The Trypanosoma brucei subspecies are morphologically indistinguishable; identification is therefore done by geographic, epidemiologic and molecular biological criteria.

The disease is vector borne which means that it is transferred from host to host via an arthropod shuttle organism, the tsetse fly (Glossina gen., Fig. 1.2). The occurrence of the vector limits the spread of the disease, hence trypanosomiasis is geographically limited to the so-called tsetse belt (1). 


\section{INTRODUCTION}

\begin{tabular}{|l|c|c|}
\hline Phylogenesis & Pathogen & Vector \\
\hline Superkingdom & Eukaryota & Eukaryota \\
Kingdom & Protista & Metazoa \\
Phylum & Flagellata & Arthropoda \\
Class & Euglenozoa & Insecta \\
Order & Kinetoplastida & Diptera \\
Family & Trypanosomatidae & Glossinidea \\
Genus & Trypanosoma & Glossina \\
Subgenus & Trypanozoon & Nemorhina \\
Species & Trypanosoma brucei & Glossina palpalis \\
Subspecies & Trypanosoma brucei gambiense & Glossina palpalis gambiense \\
\hline Fig.1.2: Phylogenetic table of Trypanosoma brucei brucei and Glossina palpalis gambiense.
\end{tabular}

The order of kinetoplastida includes many human pathogens of comparable cell biology. As for Trypanosoma, there are Trypanosoma cruzi cruzi of the Schizotrypanum subgenus. This species is the causative agent of the South American trypanosomiasis, the so called Chagas disease. In contrast to Trypanosoma brucei they are intracellular blood parasites, spread by the bite of the kissing bug (Triatominae sub family) (ㅁ). The disease displays a major burden to the continent, with an estimated 120 million people at risk and $300^{\circ} 000$ new cases each year (WHO 2004).

Another major disease, caused by kinetoplastids, is called Leishmaniasis of the Leishmania genus (9). It is spread over four continents and 88 countries with about 350 million people at risk and an estimated number of 500`000 new cases per year (WHO 2004).

The sheer number of people at risk by kinetoplastid pathogens alone shows the medical importance of trypanosome research. However, kinetoplastids are also one of the most ancient groups that are known in the eukaryote kingdom. With rRNA species that are older than those found in animals, plants and even fungi (9)), they represent a model system of ubiquitous importance for molecular biological research.

\section{LIFE CYCLE}

1. After a blood meal, division arrested trypanosomes of stumpy morphology populate the midgut of the tsetse fly. The cells differentiate into proliferating procyclic forms $P F$ and the variable surface glycoprotein VSG coat is exchanged into a procycline coat. The cells are non pathogenic to humans at this stage. After establishment in the mid gut, the cells change from trypomastigote into epimastigote morphology, with the kinetoplast positioned close to the nucleus. 
2. After successful morphogenesis, the cells migrate into the salivary gland of the fly, where they attach to the surface and change into a non proliferating, metacyclic form. The VSG coat is reestablished and the cells are ready for transfer into a new host.

3. After a bite of the fly, the cells infect the subcutaneous tissue of the new host and change into the slender type blood stream form $B S F$ of trypomastigote cells. They proliferate and migrate through the lymphatic system into the bloodstream and spread over the whole body, until the host has produced an effective antibody. The original population will decrease and produce division arrested stumpy forms, which can be transferred into a new vector, until another VSG variant will emerge and do the same. This oscillation will continue, until the trypanosomes finally invade the central nervous system and the host dies.

\section{Trypanosome life cycle}

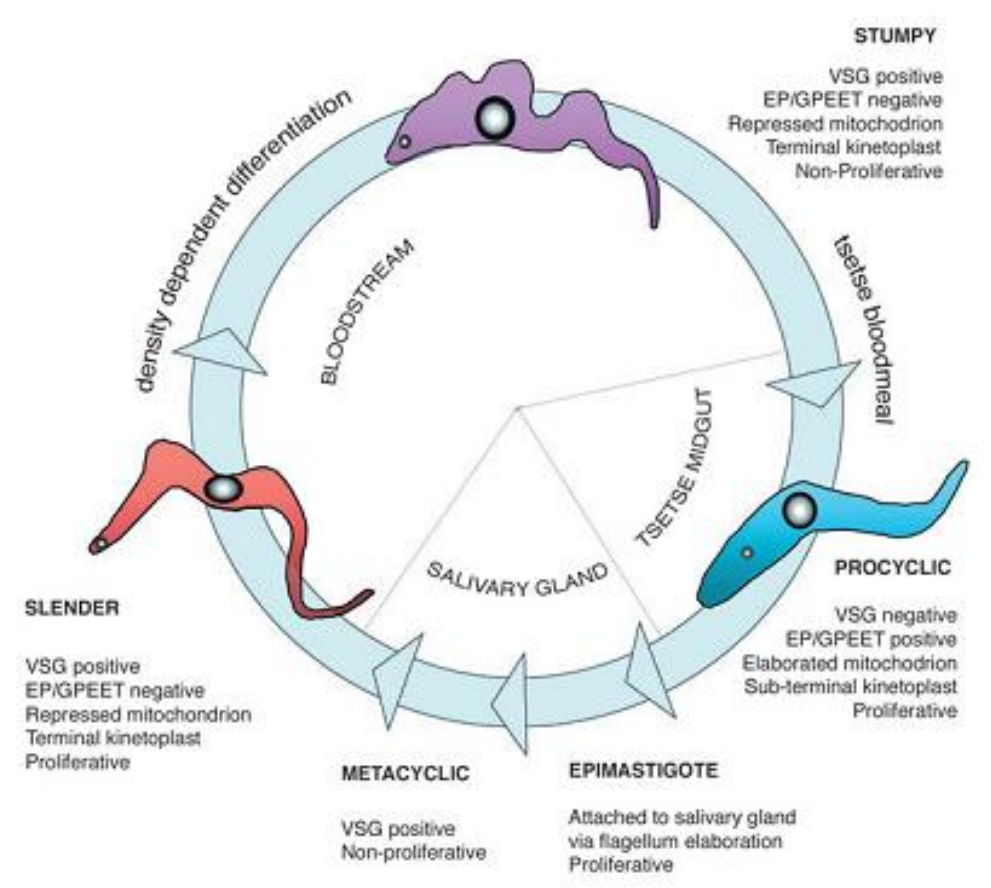

Fig. 1.3: Life cycle of Trypanosoma brucei brucei (K. R. Matthews et al. 2005). Cell morphology stage and coating are indicated. 


\section{INTRODUCTION}

\section{Cell biology}

This work concentrates on the slender type bloodstream forms of Trypanosoma brucei brucei. These trypomastigote cells have a maximum cell diameter of approximately $3 \mu \mathrm{m}$ and a total length of $20 \mu \mathrm{m}$. They mainly proliferate by fission, but sexual recombination has also been reported for procyclic cells in the vector $(\underline{10}, \underline{11})$. Bloodstream forms are constantly exposed to the host immune response and have developed a unique surface coat that helps them to withstand both antigen and complement mediated lysis. The variable surface glycoprotein VSG coat consists of a single species of about $10^{7}$ protein dimers that are anchored within the outer leaflet of the cell membrane by a glycosylphosphatidylinositol GPI anchor (Fig. 1.4).

Fig. 1.4: Trypanosoma brucei brucei cell membrane and VSG coat (M. Engstler et al. 2007).

\section{VSG and antibodies}
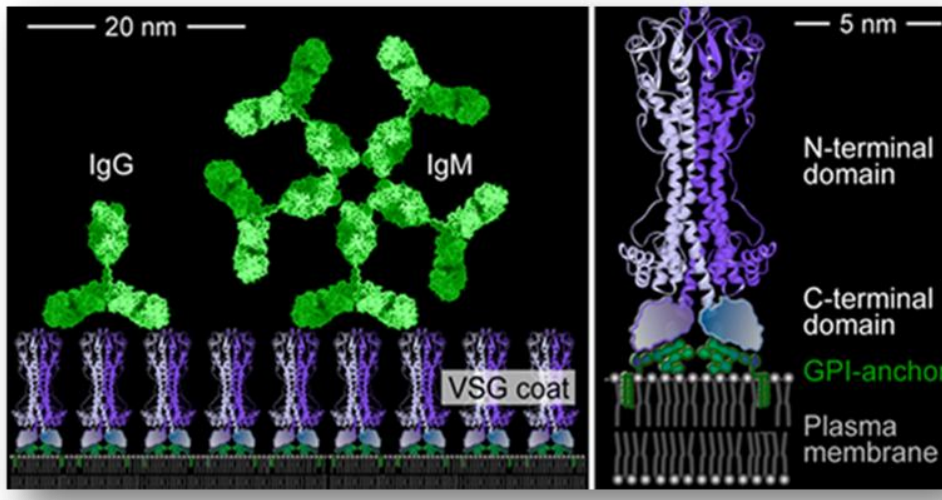

Antigenic variation is the mechanism that enables the cell to completely exchange their coating at a frequency of up to one event every 10 generations $(5-10 \mathrm{~h}$ per generation, 12$)$. If a trypanosome population is recognized by hostile antibodies, a new generation with a different

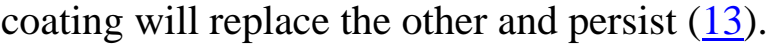

\section{flagellar pocket golgi apparatus subpellicular microtubuli}

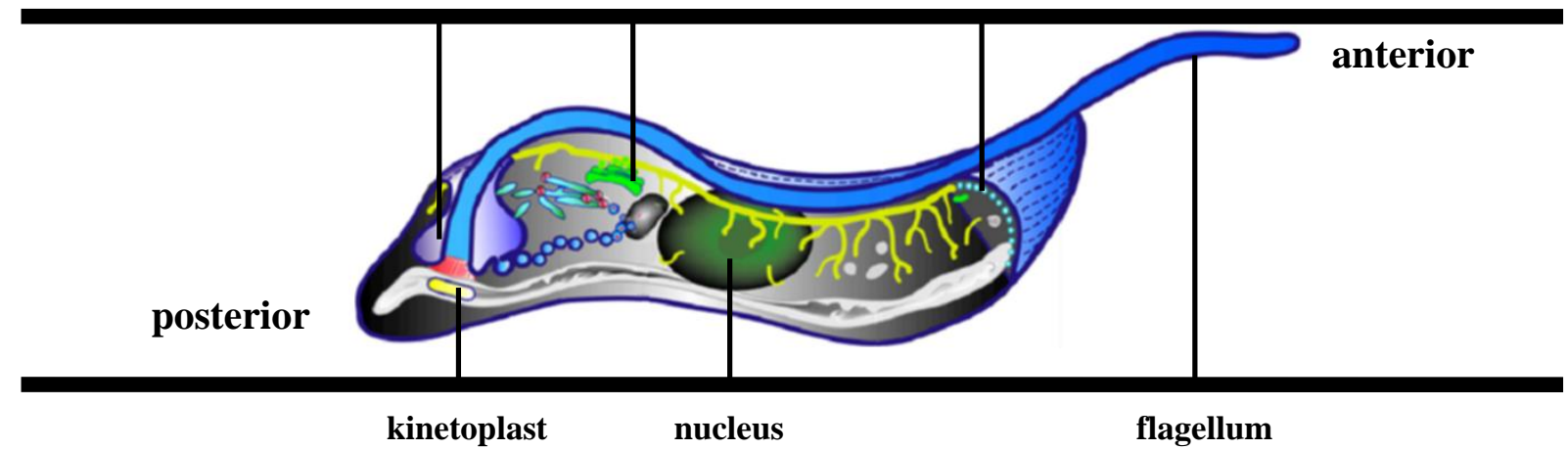

Fig. 1.5: Trypanosoma brucei brucei cell architecture of slender BSF trypomastigotes (Grünfelder et al.). 
Membrane traffic is completely dispatched via the flagellar pocket, a membrane compartment which takes up for only $5 \%$ of the cell surface $\left(145 \mu \mathrm{m}^{2}\right)$. Still the equivalent of the entire cell membrane can be exchanged within only $7 \mathrm{~min}$ via this organelle, indicating its importance for surveillance and nutrition of the cell (14).

The flagellar pocket is also the place where the flagellum emerges from the cell body. The flagellum is used for cellular propagation and anchored to the cytoskeleton via the basal body. Like the flagellum, basal bodies are comprised of microtubules and play an important role in structural cell organization and DNA separation. Moreover, it is closely associated with the kinetoplast which concentrates the entire DNA of the sole mitochondrion in trypanosomes.

The kinetoplast is a unique feature of the order marked by a very high amount of DNA, assembled to a sheet like superstructure that is comprised out of DNA mini $(1 \mathrm{~kb})$ and maxi circles $(20 \mathrm{~kb})(\underline{12}, \underline{15})$. It can easily be labeled by DNA staining methods and used to determine cell cycle and stage by its position relative to the nucleus.

The nucleus contains a diploid set of linear chromosomes and maxicircles that code for approximately 12000 intron free genes. About 1000 of those genes encode for VSG surface proteins, highlighting the paramount importance in cell survival.

\section{FLAGELLUM}

The flagellum facilitates trypanosome motility, but it is also of major importance for cell division and development. It determines structural organization and acts as a sensory organelle as well as an anchor to host tissue (16).

The structural element of the flagellum is the axoneme which is well preserved throughout the animal kingdom. The axoneme is made out of microtubules assembled in the so called 9+2 structure (Fig. 1.6) and is always $D=0.25 \mu \mathrm{m}$ in diameter (17). Two central microtubules are connected to a circle of nine outer microtubule doublets by radial spokes which are interconnected by nexin links (Fig. 1.6). The forces which deform the axoneme are generated by dynein motor proteins which induce a sliding motion between the outer microtubule doublets and the inner central pair microtubules. The conformational changes in dynein structure that cause the sliding motion are driven by hydrolysation of ATP (18), whereas in absence of ATP the flagellum will be relaxed.

The flagellum of trypanosomes is supported by a paracrystalline protein filament, called paraflagellar rod which is a unique feature to protozoan life forms. It gives structural support to the axoneme and establishes the connection to the cell body via the flagellar attachment zone (16). 


\section{Trypanosome flagellum}

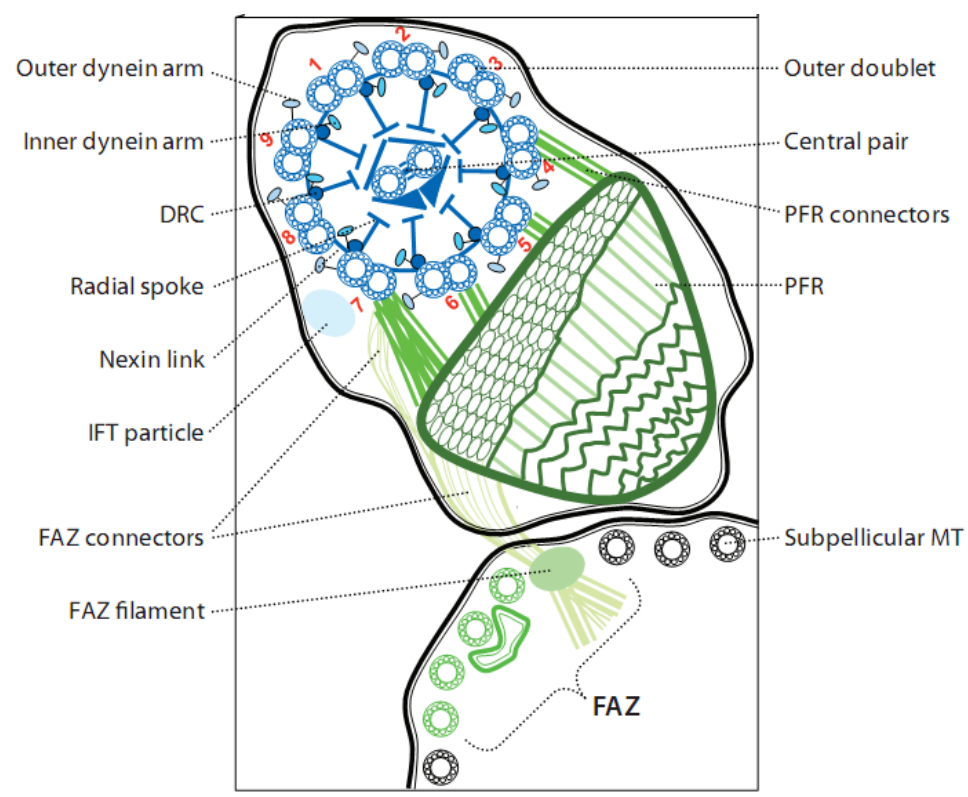

Fig. 1.6: Structure of the trypanosome flagellum and paraflagellar rod PFR (K. Hill et al. 2009).

In summary, trypanosomes represent features of the entire eukaryote spectrum within a single cell in combination with diversifications that provide new insights into old questions. We take advantage of protozoan cell culture techniques to analyze cell motility and biophysical aspects of protein sorting on trypanosomes. In doing so, we hope to gain a better understanding of both trypanosome biology and biophysical properties of living organisms in general. 


\section{MICROFLUIDICS}

Several methods exist in microfluidic device production (19). Microfluidic channels can be directly milled out of polymers or metallic substrates e.g., resulting in very rigid and precise design, but mostly limited to tenths of micrometers by the minimal size of available mills and processing times. Laser cutting and etching techniques reduced these scales to several micrometers and even nanometers in width, but those structures are limited in depth by the divergence of the focussed laser beam and- / or the length of the etching pin respectively. Another very promising technique is direct laser imprinting of $3 \mathrm{D}$ structures into polymer melts. Polymerisation is induced by laser light and the size of the features is determined by focal spot size and exposure times, producing submicrometer structures of very high aspect ratios, only limited by the rigidity of the polymer. All these techniques however are limited in processing times for larger structures. Given a voxel dwell time of a millisecond for laser induced polymerization and a focal volume of $1 \mu \mathrm{m}^{3}$, a cube with edges of $1 \mathrm{~mm}$ in length would be polymerized in only 12 days, hence such a method is not suitable for rapid production of microchannels.

We use soft lithography for rapid prototyping of microstructures in polydimethylsiloxane, PDMS (20) as a well established and advanced method in our workgroup. 2D structures are written in submicrometer resolution onto a mask which is then used to structurally illuminate a photo curable resign. Liquid PDMS is casted onto the resign master structure and the polymerized copy represents the micro channel. The depth of the channels is defined by the thickness of the resign layer, ranging from submicrometers to the millimetre scale but limited to aspect ratios of width / depth $=1 / 10$. 3D structures can be produced by subsequent addition of multiple layers, but usually do not exceed a total count of 5 layers.

PDMS is a transparent elastic polymer which is chemically inert, predetermining it for biological research where non-toxic materials are preferred and transparency is necessary for microscopic observation. Moreover PDMS can be covalently bound to glass which can be used to enclose the open channel structures with coverslips used for microscopy.

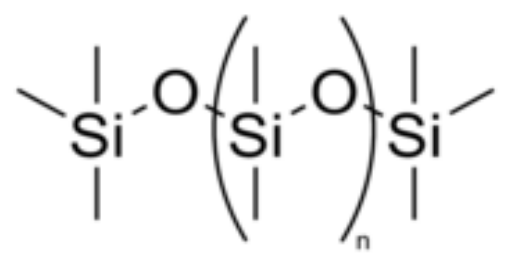

Fig. 2.1: Chemical structure of PDMS 


\section{PHOTO LITHOGRAPHY}

All lithography is done in a class 1000 cleanroom, to prevent dust particles to settle onto the micro structures. All structures are printed in Microchem SU8 permanent epoxy negative resists on pure silicon wafers $D=5 \mathrm{~cm}$.

silicon wafer

substrate

\begin{abstract}
spincoat
resist
\end{abstract}

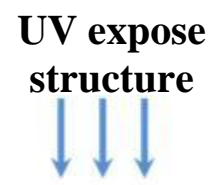

develope

structure

Fig. 2.2: Illustration of photolithography procedure using negative resists. Sequence from left to right (adapted from S. Köster 2008).

\section{Protocol: $\quad$ SU8-3050 master of $65 \mu \mathrm{m}$ height}

1. Clean wafer with isopropanol and blow dry with nitrogen

2. Activate surface in oxygen plasma for $30 \mathrm{~s}$

3. Coat wafer with approximately $5 \mathrm{~mL}$ SU8-3050 photoresist

4. Spincoat wafer at $2500 \mathrm{rpm}$ for a layer thickness of $d=65 \mu \mathrm{m}( \pm 10 \mu \mathrm{m})$

a. ramp up to $500 \mathrm{rpm}$ in $5 \mathrm{~s}$ and hold for $10 \mathrm{~s}$

b. then up to $1200 \mathrm{rpm}$ in $10 \mathrm{~s}$ and hold for $30 \mathrm{~s}$

c. then down to $0 \mathrm{rpm}$ in $5 \mathrm{~s}$ and hold for $0 \mathrm{~s}$

5. Dry bake wafer on hotplate

a. temperature ramp from $30^{\circ} \mathrm{C}$ to $95^{\circ} \mathrm{C}$ in $20 \mathrm{~min}$

b. hold at $95^{\circ} \mathrm{C}$ for $20 \mathrm{~min}$

6. Expose wafer to UV ( $\lambda=365 \mathrm{~nm}$ at $I=15 \mathrm{~mW} \cdot \mathrm{cm}^{2}$ ) for $15 \mathrm{~s}$ in soft contact mode

7. Pre post bake wafer immediately for $1 \mathrm{~min}$ at $65^{\circ} \mathrm{C}$

8. Post bake wafer for $10 \mathrm{~min}$ at $95^{\circ} \mathrm{C}$ (structures become visible)

9. Develop structure in mr-Dev600 for approximately $30 \mathrm{~s}$

10. Clean with isopropanol and blow dry with nitrogen

11. Cure 5 min at $95^{\circ} \mathrm{C}$ 


\section{PDMS MOLDING}

Lithography, in combination with PDMS molding, also called soft lithography, allows for rapid prototyping both in microfluidic design and production of actual microfluidic devices (20). The imprinted structures on the mask are designed using AutoCAD software (Materials) and printed either in chromium on quartz glass or with ink on polymer substrates in less than a week, while fabrication of the resin master is a matter of hours. Once a master has been produced it can be copied in PDMS several dozen times and the actual microfluidic device is ready to use within 3 hours, including baking time.

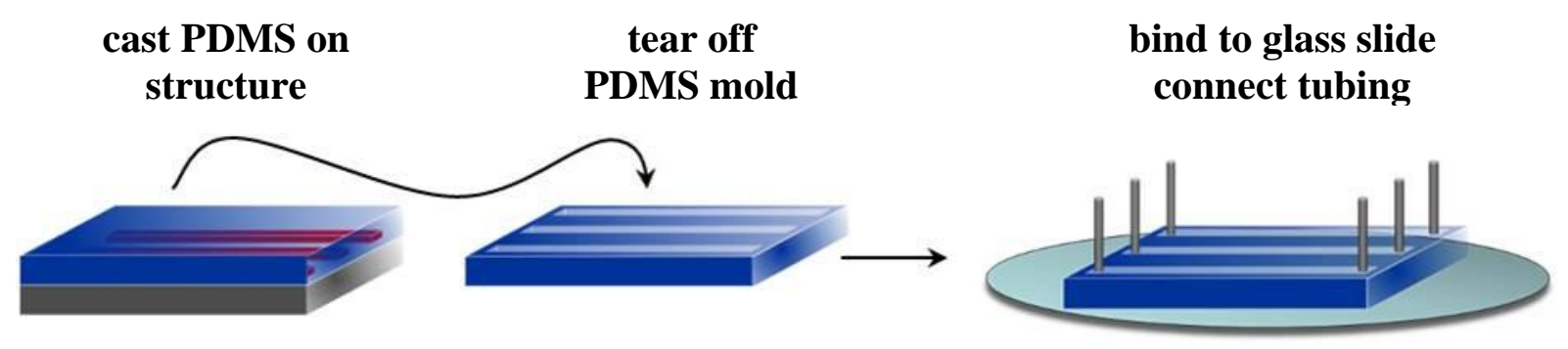

Fig. 2.3: Illustration of PDMS molding procedure using negative resists (adapted from S. Köster 2008).

\section{Protocol: $\quad$ PDMS molding and assembly of the device}

1. Add $1 / 10 w / w$ parts of curing agent to PDMS polymer (10 g PDMS per wafer)

2. Cast PDMS onto structure and degas in vacuum for $30 \mathrm{~min}$

3. Bake PDMS $3 \mathrm{~h}$ at $75^{\circ} \mathrm{C}$

4. Cut out structure of interest and push tubing holes into PDMS

5. Rinse coverslip with isopropanol and air dry with nitrogen

6. Expose both glass and PDMS to oxygen plasma at 2 mbar pressure for $30 \mathrm{~s}$

7. Quickly attach PDMS replica to the coverslip and support binding with gentle pressure

8. Connect tubing

9. Rinse channels with isopropanol

10. Post bake device for $30 \mathrm{~min}$ at $75{ }^{\circ} \mathrm{C}$

The tubing connection might be enforced using glues, but usually press fit connections fit tight if the diameter of the pushing tool is about $20 \%$ smaller than the outer tubing diameter. Rinsing of the channel helps deactivating the surface, however several methods are documented to modify the inner surface properties $(\underline{21}, \underline{22})$. 


\section{PRECISION OF METHOD}

In order to determine the precision of lithography and PDMS molding, both resin masters and the PDMS copies have been analyzed exemplary, using optical, scanning electron and atomic force microscopy methods. Direct measurement of the coated wafer height using a micrometer scale, revealed deviations as big as $20 \%$ of total height over the entire area of the wafer. However, the structures of interest usually do not exceed areas bigger than $4 \mathrm{~cm}^{2}$ and deviations analyzed with reflective laser scanning methods show deviations smaller than $10 \%$ of the total (Fig. 2.4 a).

The lateral resolution is usually better and has been examined by a cross section of a PDMS channel as seen in the microscope (Fig. 2.4 b).

\section{Confocal laser scan}

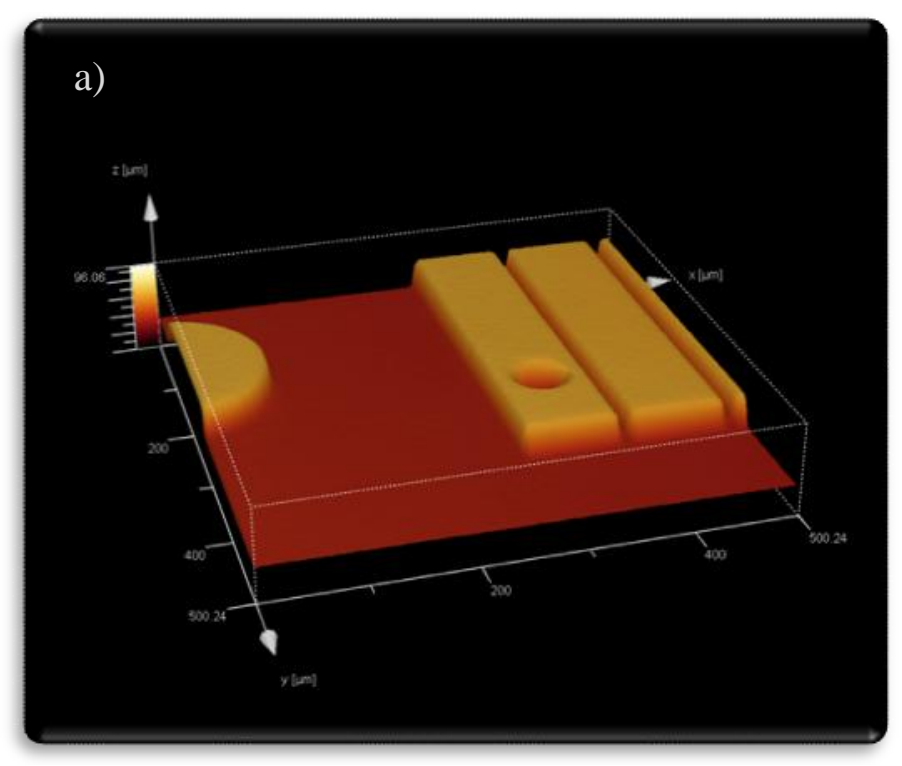

\section{PDMS}

cross-section

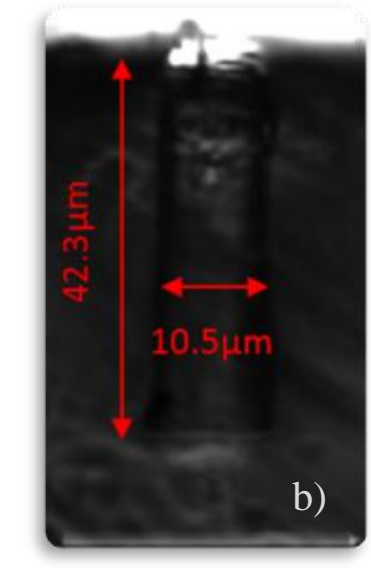

Fig. 2.4: a) Reflective confocal laser scan of $10 \times 43 \mu \mathrm{m}$ microchannel master (Leica SP2, $\lambda=542 \mathrm{~nm}$, 10x/0.3 Leica HC PL Fluotar objective). b) Cross-section of the PDMS replica.

The lateral precision is about $5 \%$ of the expected value (Fig. 2.4 b) at an aspect ratio of $1 / 4$ $w / h$ for long perpendicular structures. Resolution decreases with smaller structures like circles e.g. as well as with increasing aspect ratio. We found that aspect ratios higher than $1 / 5$ fail to be resolved properly in our setup for all used SU8 photoresists. Modified methods and resists are provided by MicroChem Corp for higher aspect ratios if needed.

High precision measurements on lateral resolution were conducted by scanning electron microscopy (SEM) and are shown in figure 2.5. The SEM images were taken at the Chemistry Department of the University of Hamburg. 
SEM scanning electron microscopy images of resin positive
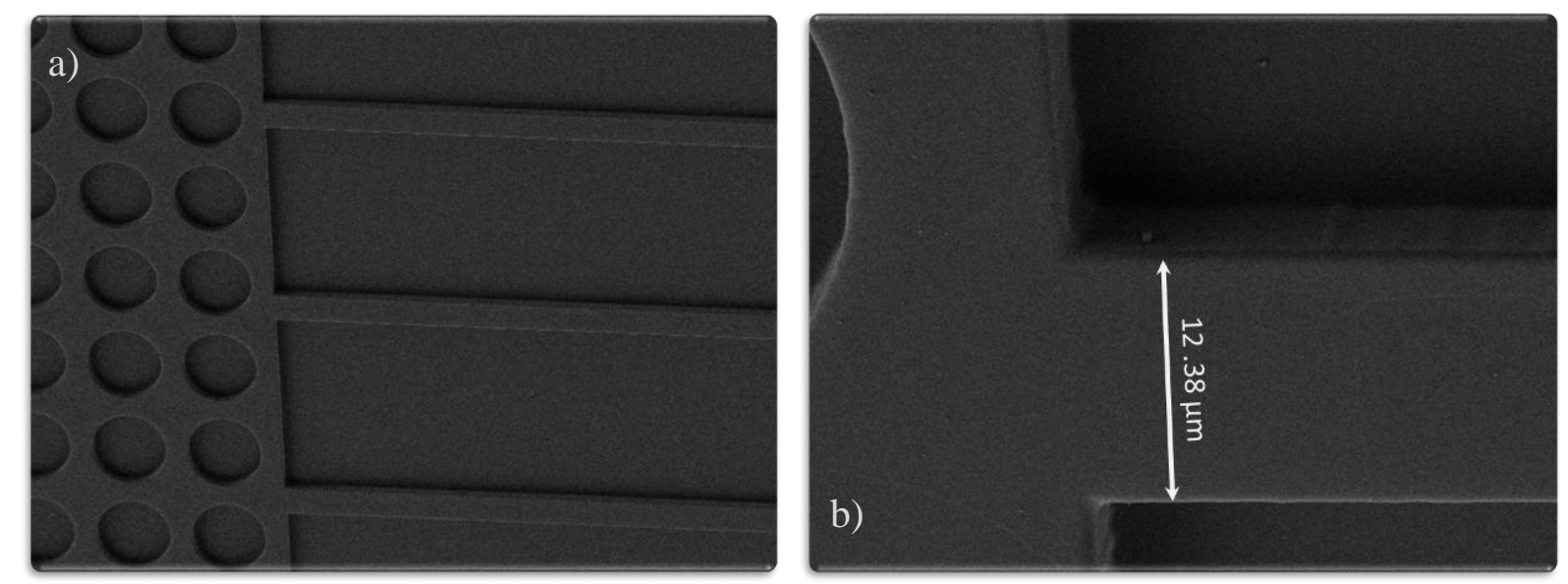

Fig. 2.5: a) SEM micrograph on resin structures. b) Zoom in with scale (white arrow)

Resolution of ordinary photolithography is limited by diffraction. The smallest channels we produced for our experiments were $1 \mu \mathrm{m}$ in width. We analyzed channels of $w \geq 3 \mu \mathrm{m}$ width by atomic force microscopy AFM (Fig. 2.6) to get a better estimate of the precision of channels smaller than $w \leq 10 \mu \mathrm{m}$ in width.

\section{AFM measurements}
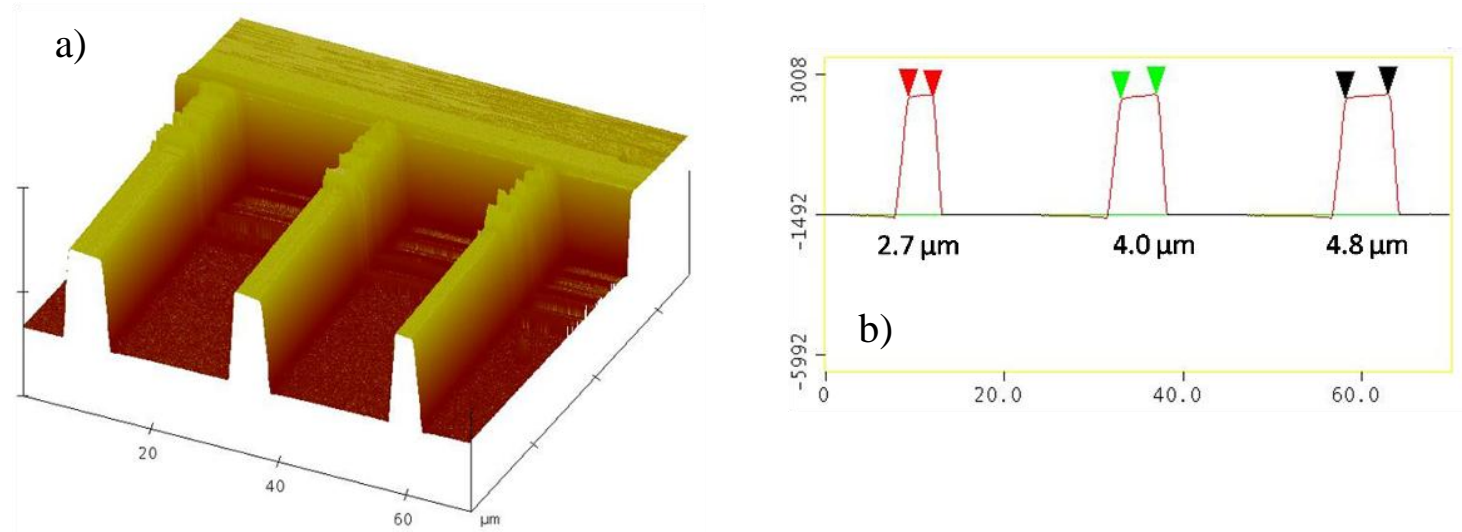

b)

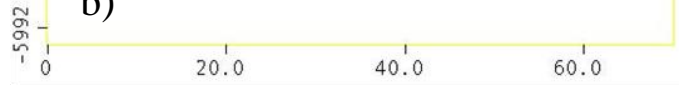

Fig. 2.6: a) AFM image of $w=3,4,5 \mu \mathrm{m}$ channels. b) Determination of channel width. Red arrows: $3 \mu \mathrm{m}$ channel; green arrows: $4 \mu \mathrm{m}$ channel; black arrows $5 \mu \mathrm{m}$ channel.

The channel widths of the resin masters are in very good agreement with our expectations (Fig. 2.6, 2.5). PDMS molds are known to reproduce single nanometer features. With respect to our channel dimensions and the flexibility as well as the mechanic stresses during assembly, we estimate a worst case structure precision of $w \geq 1 \mu \mathrm{m}$ with aspect ratios $\leq 1 / 4$ of $\pm 0.5 \mu \mathrm{m}$. 


\section{MICROFLOW}

Generation of constant microflow at low velocities is necessary for cell motility investigations, using optical tweezers which are limited in stall force. Real time labeling of trypanosomes has been conducted with different reactants in co-laminar flow. Figure 2.7 shows a typical setup for such an experiment, with an optical trap integrated into the microscope epi-fluorescence pathway.

\section{Microfluidic setup}
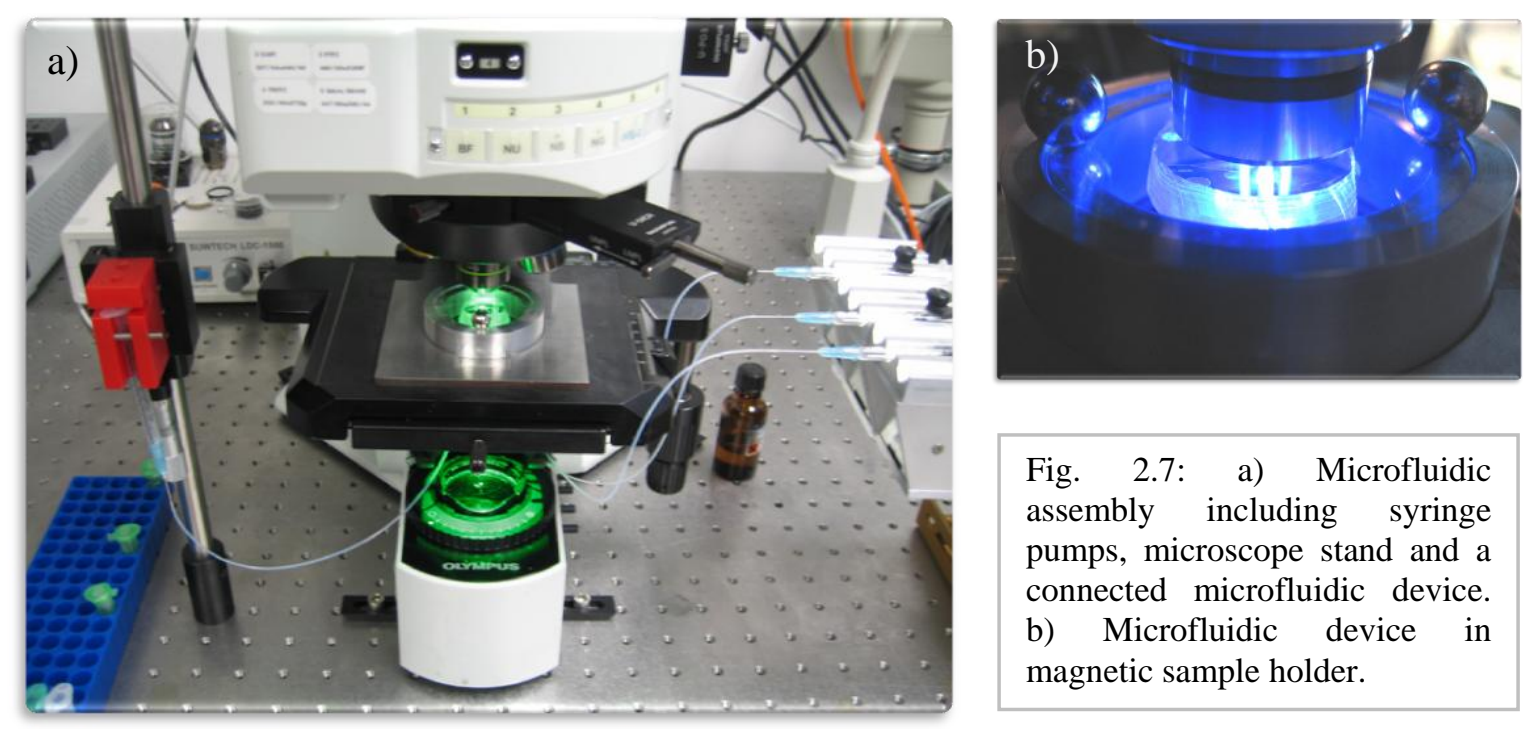

Fig. 2.7: a) Microfluidic assembly including syringe pumps, microscope stand and a connected microfluidic device.

b) Microfluidic device in magnetic sample holder.

A focus of three laminar fluids is shown in figure 2.8. The flow velocity has been precisely measured using tracer particles, in both brightfield illumination or fluorescence excitation.

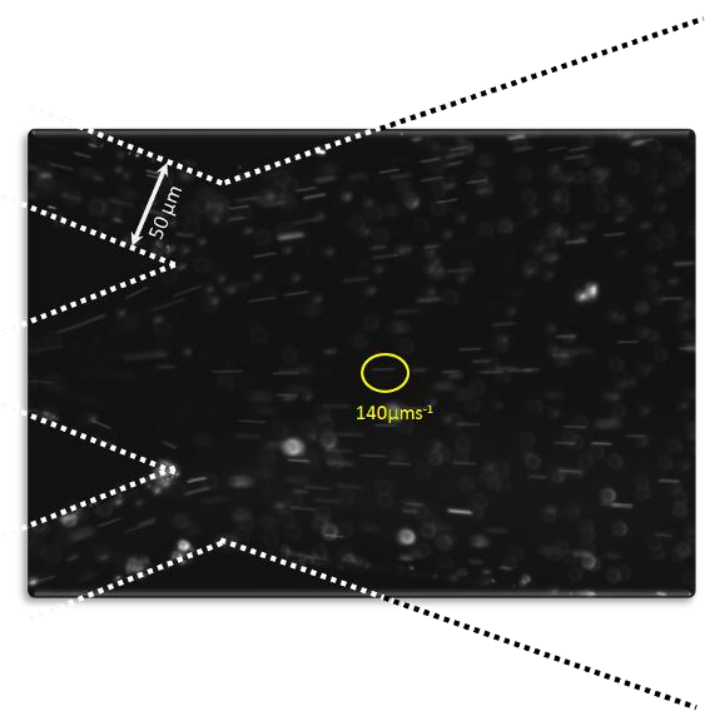

\section{Triple laminar fluid focus}

Fig. 2.8: Laminar focus of three different fluids containing fluorescent tracer particles. Yellow circle: particle streak as flow velocity indicator.

The flow velocity in the center of the triple fluid focus is $v=140 \mu \mathrm{m} \cdot \mathrm{s}^{-1}$ given by the tracer streak, highlighted in yellow. The minimum flow velocity established in this setup for a constant flow without disturbances was $v=30 \mu \mathrm{m} \cdot \mathrm{s}^{-1}$, sufficiently low for optical trapping and transporting of trypanosomes from one fluid flow to the other. 


\section{STOP FLOW}

Constant flow velocities are crucial e.g. for calibration of optical stall forces, or generation of diffusive gradients between different co-laminar fluids. It is necessary to use low friction syringes with precise pumps and to remove any air that might be trapped within the channel in order to create such conditions. However, it has been proven that it is even more difficult to obtain good stop flow within ordinary microchannels of small dimensions. We developed a microfluidic stop flow device with channels of different widths, oriented perpendicular to the pressure gradient of the inlets in order to maintain stop flow within those channels.

\section{Triple laminar fluid focus}

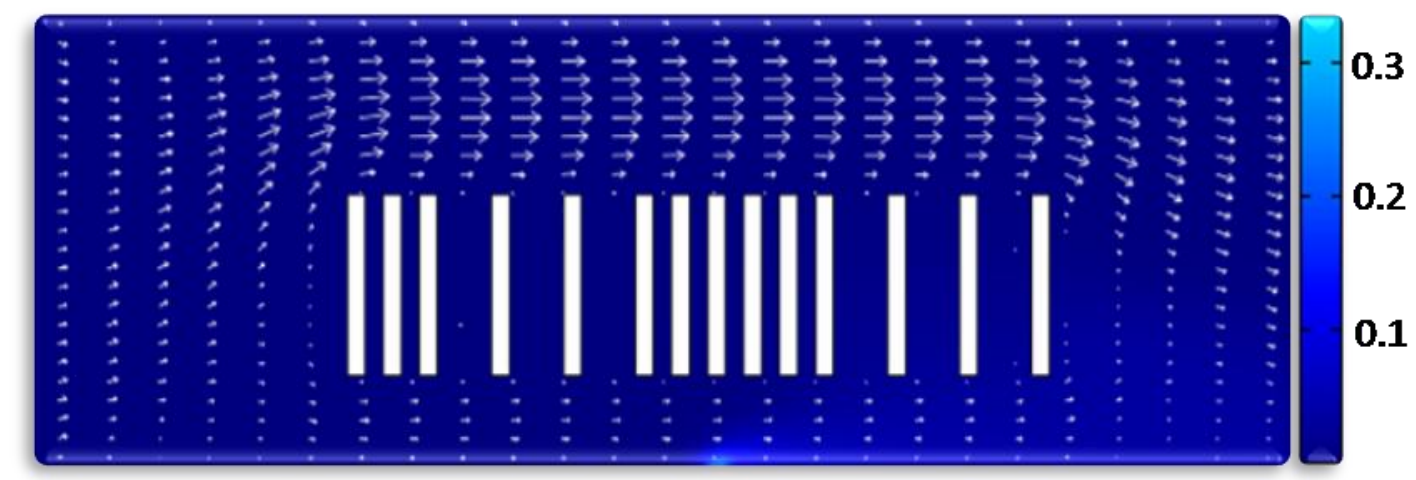

Fig. 2.9: Finite elements simulation (Comsol Multiphysics 3.5) of fluid flow within a microfluidic stop flow device. Flow direction is from left to right. The scale gives the flow velocity in relative numbers. The white arrows indicate flow velocity and direction. An additional structure, connected at the lower part of the simulation, induces a small disturbance of flow it has been removed from the image since it is not relevant.

The simulation predicts a complete absence of flow within the perpendicular channels, even with flow on the outsides. Inducing different fluids above and below the perpendicular channels facilitates the generation of very shallow diffusive gradients that can be dynamically modulated by fluid velocity, whilst stop flow is maintained along the gradient in the channel of interest. This kind of setup is used for the analysis of trypanosome motility in confinement (chapter 4.3) and will be used for the study of chemotaxis of cells in future experiments to come.

With the presented methods at hand, we can study the life of trypanosomes at low Reynolds numbers and in spatial confinement, both with and without flow. 


\section{THEORY OF OPTICAL TRAPPING}

The principle of optical trapping is based on the interaction of matter with light. Light itself carries a mass and transfers a momentum if being diffracted, reflected or absorbed. The easiest way to imagine optical trapping is playing billiards with light and very small particles.
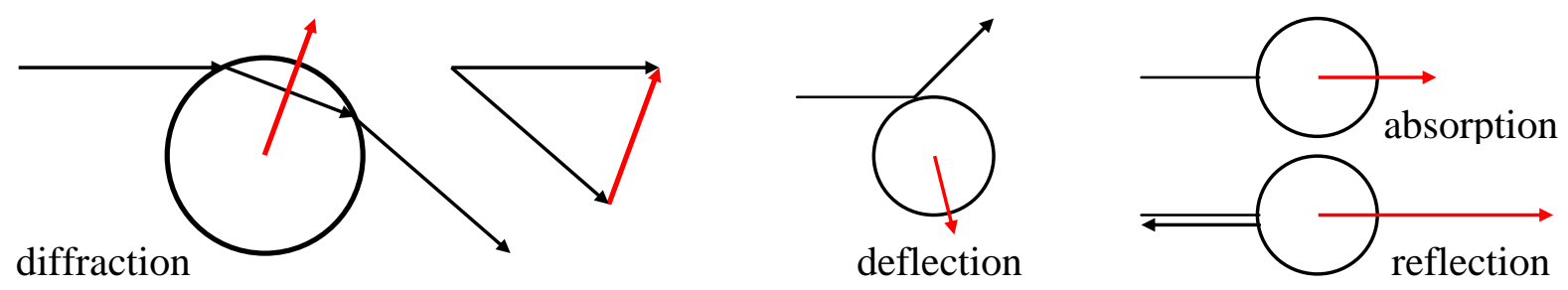

As described in Newton's second law of classical mechanics, a force $F$ is the product of a mass $m$ that is being accelerated by a factor of $a$

$$
F=m a
$$

and a mass that is moving at a constant velocity $v$ carries a momentum $p$ that can be transferred to another mass given by

$$
p=m v
$$

Using Einstein's interpretation of the Planck's action quantum $h=6.626^{*} 10^{-34} \mathrm{Js}$ describing the photoelectric effect, every photon has a discrete energy and momentum. With $\lambda$ being the wavelength, the momentum of a photon is given by

$$
p=\frac{h}{\lambda}
$$

The momentum of a single photon is very small indeed, so one needs very many of them to effectively displace even a micron sized particle. Only the invention of light amplified stimulated emission of radiation laser, allowed for producing a sufficiently strong momentum. With the energy of a photon given by

$$
E=\frac{h c}{\lambda}
$$

with $c$ as the velocity of light, the momentum exceeded on a surface can be calculated and probed in experiment. 
Already in 1619 the German astronomer Johannes Kepler (23) postulated the comets tail, always pointing away from the sun, would be driven by radiation pressure. In 1900 this concept could firstly be proven experimentally by the Russian physicist Pyotr Nikolajewitsch Lebedev and precisely measured with the Nichols radiometer invented by Ernest Fox Nichols and Gordon Ferrie Hull in 1901.

But it was only in $1971(\underline{24})$ with the development of laser light sources, when Arthur Ashkin could show that radiation forces can actually be utilized, to effectively manipulate microscopic particles. With the observation of particles being accelerated along the propagation axis of a focussed laser beam, Ashkin also realized a traverse force towards the centre of the beam and he conducted further experiments to reveal the cause of this effect.

Using monodisperse polystyrene spheres dissolved in water, a particle should not experience any directional force within a homogeneous force field, but within a gradient there should be an attractor. These experiments gave rise to the ray optics theory of optical trapping. In a two counter propagating laser beam setup, with the light passing through water, it was possible to optically confine a polystyrene sphere in water and levitate a $20 \mu \mathrm{m}$ glass sphere against gravity in air, using a $250 \mathrm{~mW} \mathrm{TEM}_{00}$ mode laser (24).

It was also Arthur Ashkin who developed the single beam gradient trap using high numerical aperture objectives to highly focus a laser beam and produce a three dimensional trapping potential in $1986(\underline{25})$.

\section{Two beam trap}
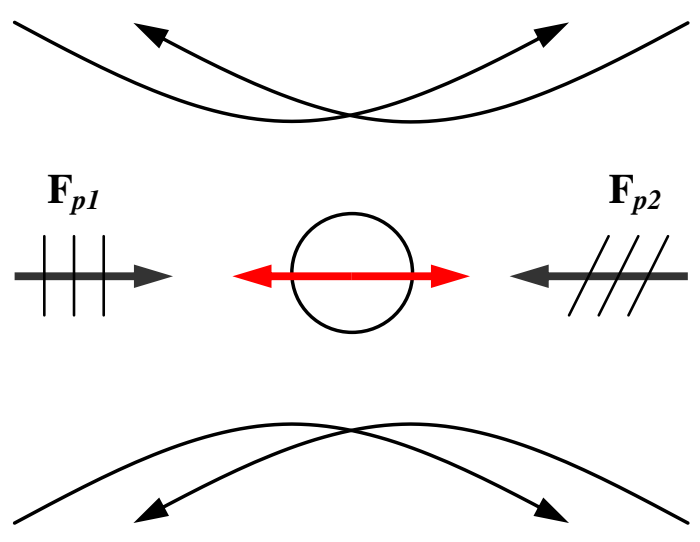

levitation trap

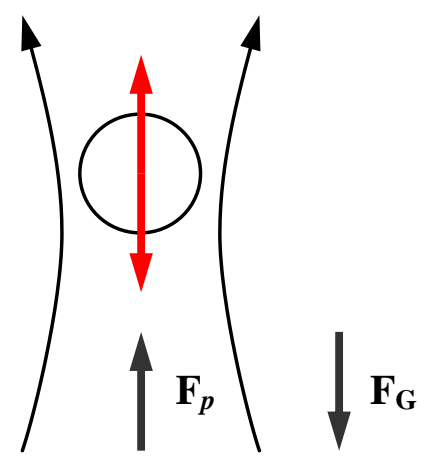

Fig. 2.10: Two optical trapping realizations by Arthur Ashkin. Left: Two cross polarized counter propagating laser beams with a polystyrene sphere in its force equilibrium position in water. Right: One beam optical levitation trap, where the radiation pressure is leveled against the gravitational force of a glass 


\section{single beam gradient trap}

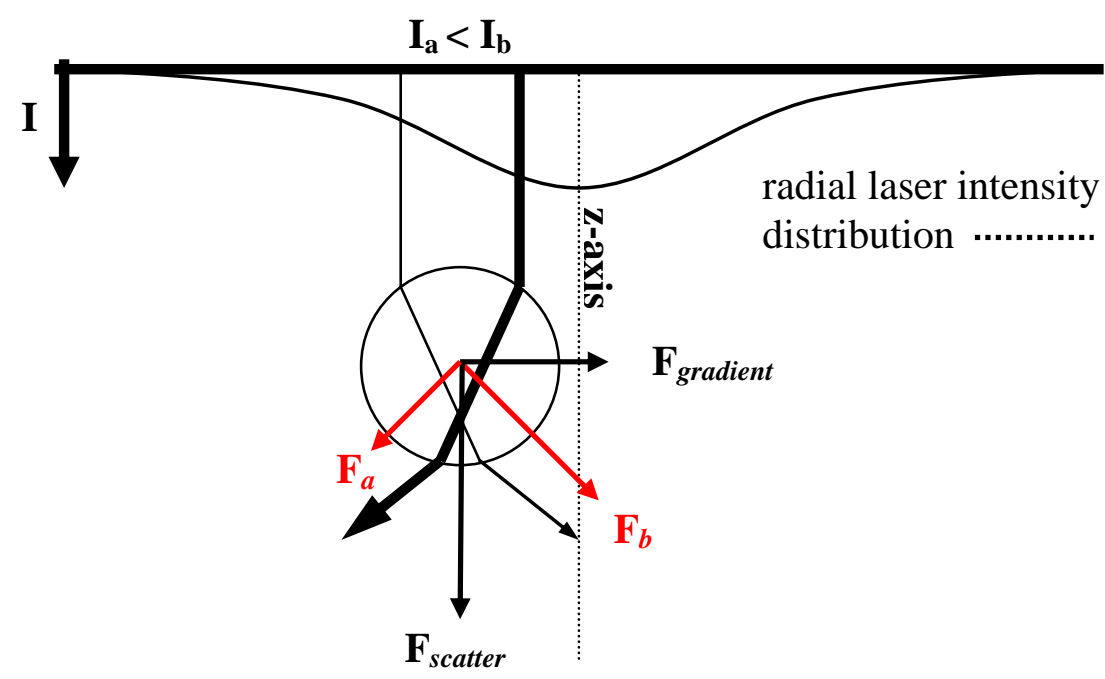

Fig. 2.11: Single beam gradient trap by Arthur Ashkin. Black curve indicates Gaussian laser beam intensity distribution. Black arrows give the propagation direction of the photons whereas the red lines indicate the resulting momentum.

One of the big advantages of this technique is that it is possible to manipulate basically everything you can see under the microscope, without actually touching it with mechanical tweezers or similar tools. This is the reason, why the optical trap has first been named "optical tweezers". However, one of the most important things about this technique is its "weakness": The optical stall force is proportional to the laser power. To exert pico-Newton forces on a spherical particle, you need several milliwatt of laser power. In an undisturbed experimental setup, one can measure forces acting on nanometer sized spheres with pico-Newton precision, simply by varying the intensity of laser light by several milliwatt. This technique allowed access to a whole new regime of force measurements on all kinds of matter. Up till now optical traps have been developed to:

- manipulate dielectric particles on the micro and nano scale

- measure pico- and femto-Newton forces on these particles

- manipulate living cells in biology

- measure mechanic properties of biopolymers and living cells

- do rheology experiments in micro

- do micro fertilization experiments

- do cell sorting

- $\quad$ super cooling single atoms and many more $(\underline{26})$ 
We constructed an optical trapping fluorescence microscope to catch and manipulate trypanosomes within microfluidic environments, analyze their motility and measure the forces the cells can generate by their flagellum.

In order to measure forces exerted on a particle by a single beam optical trap, it has to be calibrated. This is achieved by a comparison of the normal Brownian motion of a particle with the damped motion it undergoes in an optical potential, as produced by the optical trap. In thermodynamic equilibrium, the diffusion constant $\mathrm{D}$ is given by the Einstein relation

$$
D=\frac{\kappa_{\mathrm{B}} \cdot T}{\zeta}
$$

with the Boltzmann constant $k_{B}=1.38^{*} 10^{-23} \mathrm{~J} / \mathrm{K}, T$ as temperature in Kelvin and the friction coefficient $\zeta$ (for a sphere) given by the Stokes equation

$$
\zeta=6 \pi \eta r
$$

The scattering force in a medium of index $n_{b}$ in terms of the intensity $\mathrm{I}_{\mathrm{o}}$ and effective refractive index $m$ is given by (24)

$$
F_{\text {scatt }}=\frac{I_{o} 128 \pi^{5} r^{6}}{c 3 \lambda^{4}}\left(\frac{m^{2}-1}{m^{2}+2}\right)^{2} n_{b}
$$

and the gradient force in the direction of the intensity gradient for a given polarization of $\alpha$ is

$$
F_{\text {grad }}=-\frac{n_{b}}{2} \alpha \nabla E^{2}=-\frac{n_{b}^{3} r^{3}}{2}\left(\frac{m^{2}-1}{m^{2}-2}\right) \nabla E^{2}
$$




\section{OPTICAL TRAP SETUP}

In order to construct a precise and versatile yet cost effective optical trapping microscope, one has to take into account several basic considerations about its components, mainly the laser, the objective and the detection system. Amongst these, damping of the system, including low frequency vibrations, sound and stop flow conditions within the microfluidic environments to be used, are of crucial importance. The same is true for all the optics included in illumination, fluorescence, beam steering and modulation.

\section{THE LASER}

The laser needs to be strong enough to efficiently trap particles, but it also needs to be of good beam quality. The intensity distribution of the laser must be as close to a Gaussian distribution as possible to generate a strong gradient force attracting the particle and a diffraction limited focal spot, determined by the numerical aperture of the objective. This characteristic feature is called the $M^{2}$ factor and should be equal or smaller than 1.2. The $M^{2}$ factor is given by:

$$
M^{2}=\theta \cdot \frac{\pi \cdot \omega_{0}}{\lambda}
$$

where $\omega_{0}$ is the beam radius at the beam waist, $\lambda$ is the wavelength and $\theta$ is the half angle beam divergence. In other words, a $M^{2}$ factor of 1 is diffraction limited and is a perfect Gaussian beam, whilst smaller values are not possible (27).

Nevertheless the $\mathrm{M}^{2}$ factor is not the only factor describing the characteristics of the laser beam. Intuitively there must be absolutely no pointing instability to keep the trapped particle at a well defined position at the sub-nanometer scale within the objective lateral focus. Also, whilst passing all the included optics, the wavefront might suffer distortions, eventually leading to interference effects over the given pathlength. Therefore it is mandatory to keep pathlengths short and the number of optical compounds as small as possible.

Last but not least, the wavelength needs to be chosen well for the particular application. High quality neodymium-doped YAG $\mathrm{Nd}^{3+}$ :YAG lasers are commonly available for reasonable pricings at $1064 \mathrm{~nm}$, but the absorption at that wavelength is already high in water and in biological matter as well. Quantitative studies about phototoxicity over trapping wavelength in E. coli and eukaryotic cells have been conducted by Steven M. Block et al. (228) and Liang et al. (29) and are compared in figure 2.12. 


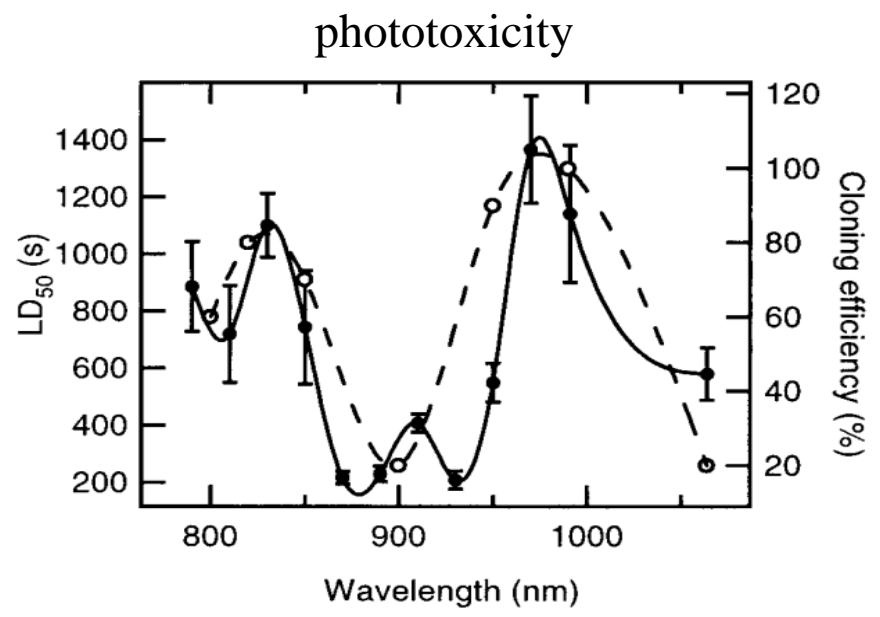

Fig. 2.12: The dotted line displays the action spectrum of rotating $E$. coli as $\mathrm{LD}_{50}$ (half maximum rotation rate) compared to the cloning efficiency of Chinese hamster ovarian cells $\mathrm{CHO}$ depicted by the solid line. Both show maxima of vitality at 830 and $970 \mathrm{~nm}$, when phototoxicity is highest at 930nm and 1064.

Diode laser are electrically pumped semiconductor laser that are widely used in data storage application and telecommunication, therefore they are subject to the rapid development of microelectronics. Mass production leads to relatively low cost, but high quality laser diodes that are commercially available, emitting at different power levels and in various wavelengths. Efficient optical trapping needs sufficient laser power, starting at $5 \mathrm{~mW}$, where a bacterium can be trapped, going up to $50 \mathrm{~mW}$ for rapidly moving eukaryotic cells with several micrometers in size. Considering photodamage as discussed above (Fig. 2.13), we choose a Sanyo 808nm laser diode, which emits $200 \mathrm{~mW}$ of laser light in single mode distribution.

\section{Sanyo VPSL 808-200 power spectrum}

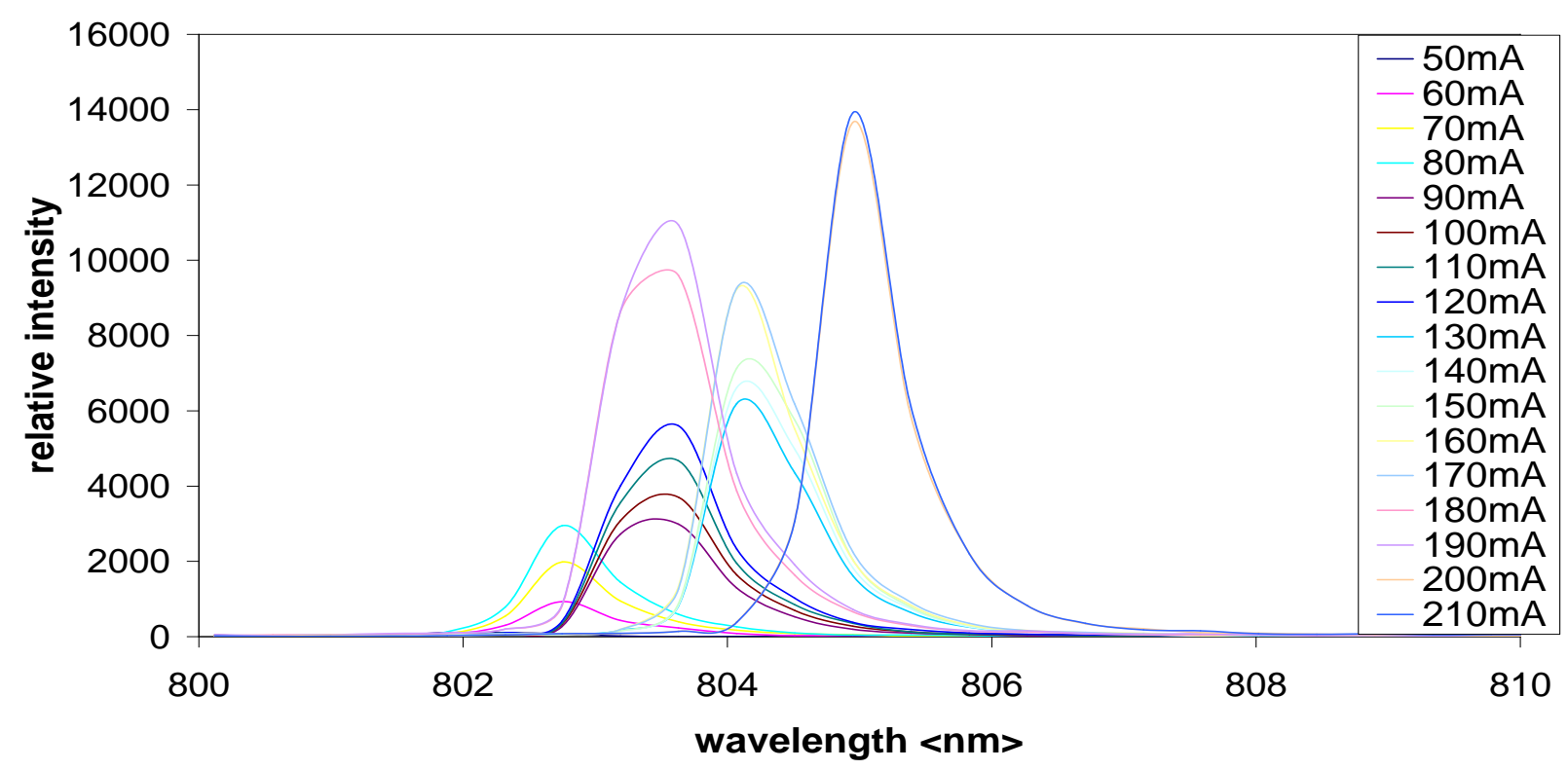

Fig. 2.13: Blue Sky Research Circulase Sanyo VPSL 808-200 sm laser diode power spectrum recorded with ocean optics HD 2000 spectrometer. The diode current was controlled using an ILX lightwave LDX-3500 driver. 
As a geometric property of the ID resonator, the electromagnetic emission is coherent in phase, but divergent and elliptical in XY propagation direction. The chosen Sanyo virtual point source VPSL laser diode is corrected for ellipticity by inclusion of diffractive optics and shows very little astigmatism, hence its beam quality is diffraction limited and the wavefront evenly spherical.

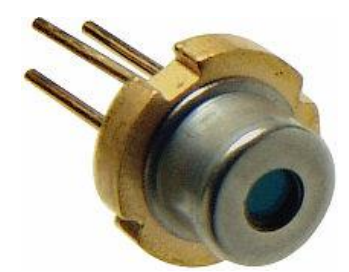

Fig. 2.14: a.) $5.6 \mathrm{~mm}$ closed can laser diode b.) Basic structure of a semiconductor laser diode assembly. Beam ellipticity is caused by the asymmetric geometry of the emitting active layer.

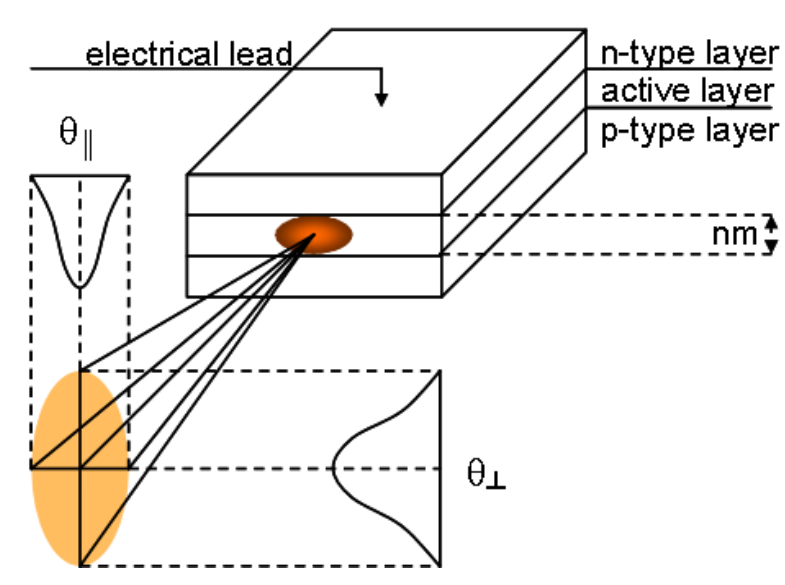

A priori divergent laser beam can be collimated using only a single lens without the need of a telescope assembly, which reduces optical distortion, simplifies adjustment and generates a steady, diffraction limited laser source for a reliable high resolution optical trap.

\section{Laser beam profile}
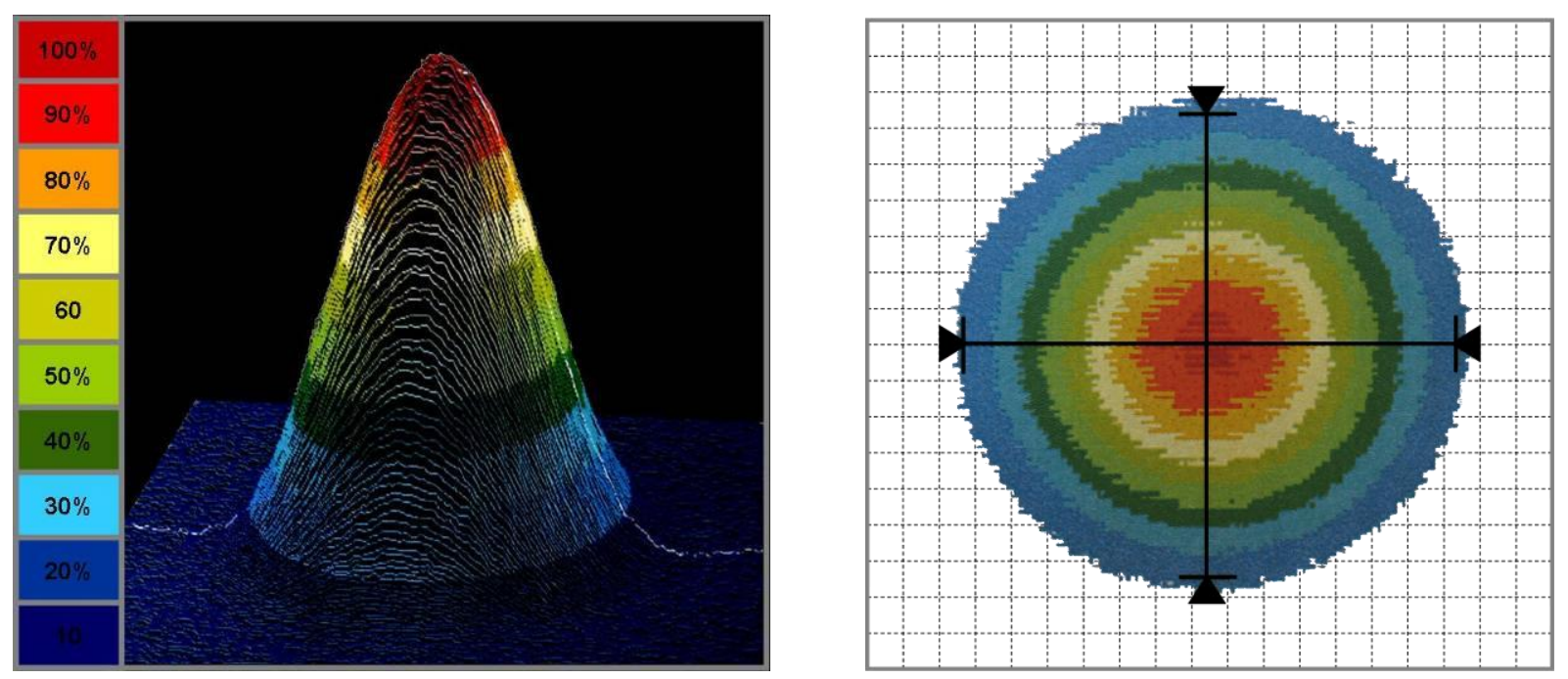

Fig. 2.15: upper graph: Sanyo VPSL 808-200 sm laser diode beam profile after collimation within Schäfter \& Kirchhoff 55BC module as specified by company. 
Laser beam profile

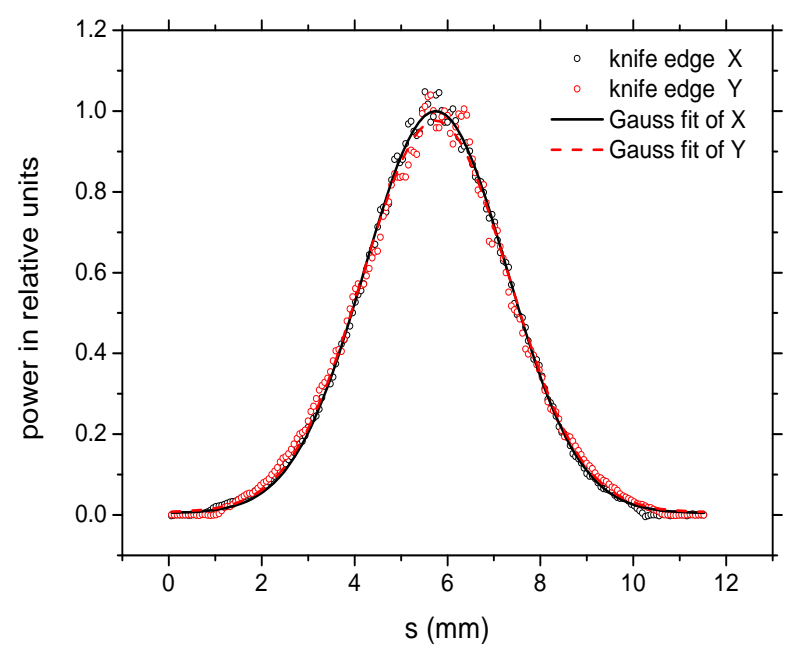

Fig. 2.16: In house knife edge characterization of the same laser diode. All data normalized by maximum of $\mathrm{X}$ Gauss fit. FWHM X: 3.61mm Y: 3.69; Ellipticity: 1.02; knife edge translated with Sutter MP385 micromanipulator, power measured with Thorlabs PM100D photometer

Calibration of the optical forces, exerted by the laser beam, is necessary to finally measure pico-Newton forces on the sample. Apart from the light source itself, the power supply needs to be very reliable over different time scales. The power drift over short and long time scales has been measured after all included optics at the focal plane of the objective. A well designed optical trap would produce a cohesion force of about $10 \mathrm{pN}$ on a $2 \mu \mathrm{m}$ polystyrene bead in water, at a lasing power of $10 \mathrm{~mW}$ at the sample. A light intensity of $10 \mathrm{~mW}$ at the sample would result of a $120 \mathrm{~mA}$ driving current and the scaling is linear, so a deviation of $1 \%$ in laser output power would add an error of $0.1 \mathrm{pN}$ to the force measurement.

\section{THE POWER SUPPLY}

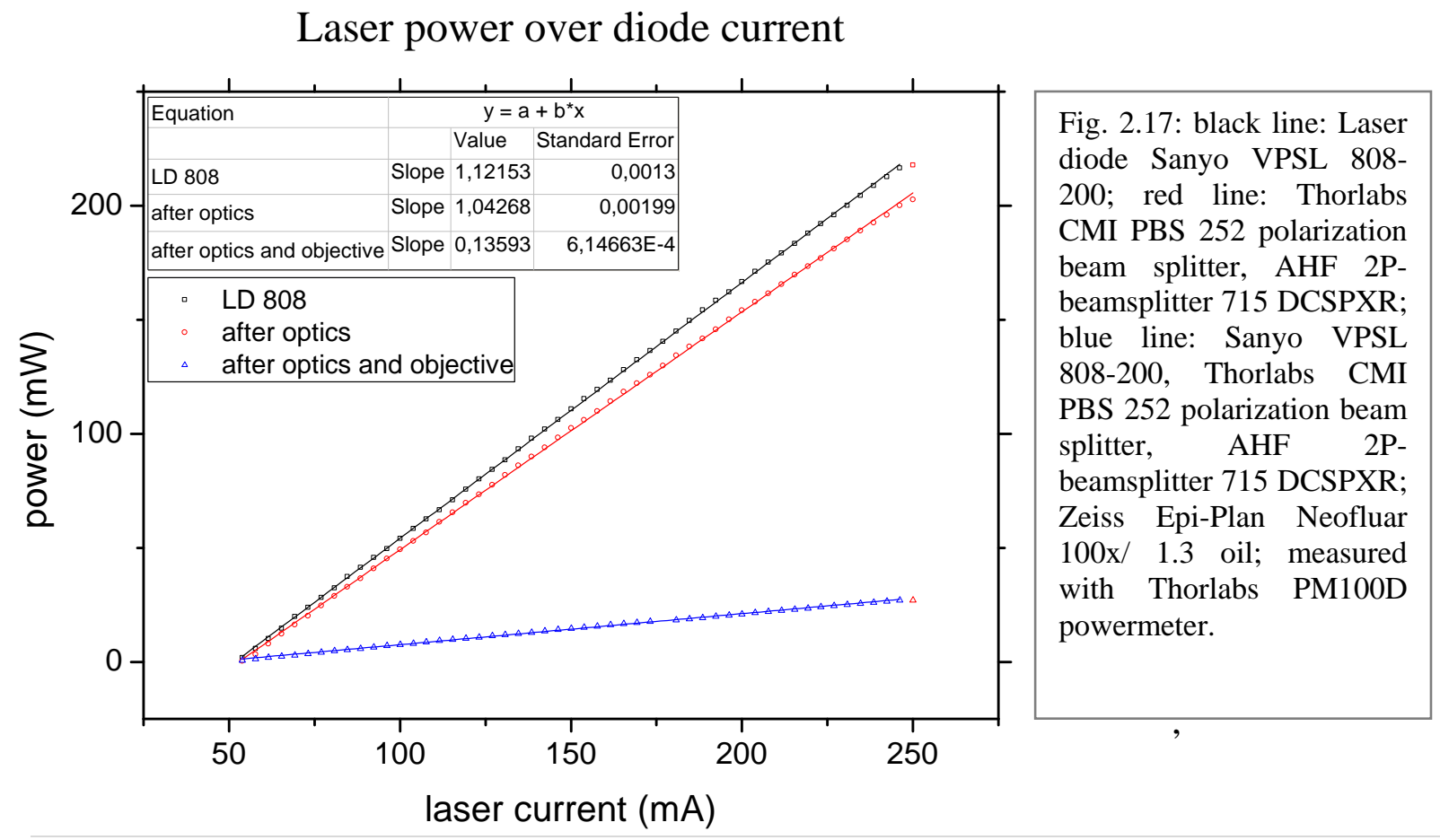


Different power supplies have been used for the setup. The ILX lightwave LDX-3500 driven laser output varied as much as standard deviation $\mathrm{STD}=0.37 \mathrm{~mW}(2.6 \%)$ over $12 \mathrm{~h}$ and was replaced by a Wavelength Electronics FL593D diode driver board which only varied about $\mathrm{STD}=0.04 \mathrm{~mW}(0.3 \%)$ over $12 \mathrm{~h}$. Additionally the FL593D is a dual driver board which two channels can be controlled via TTL signal independently and data logging is possible via USB PC connection.

\section{laser power drift}

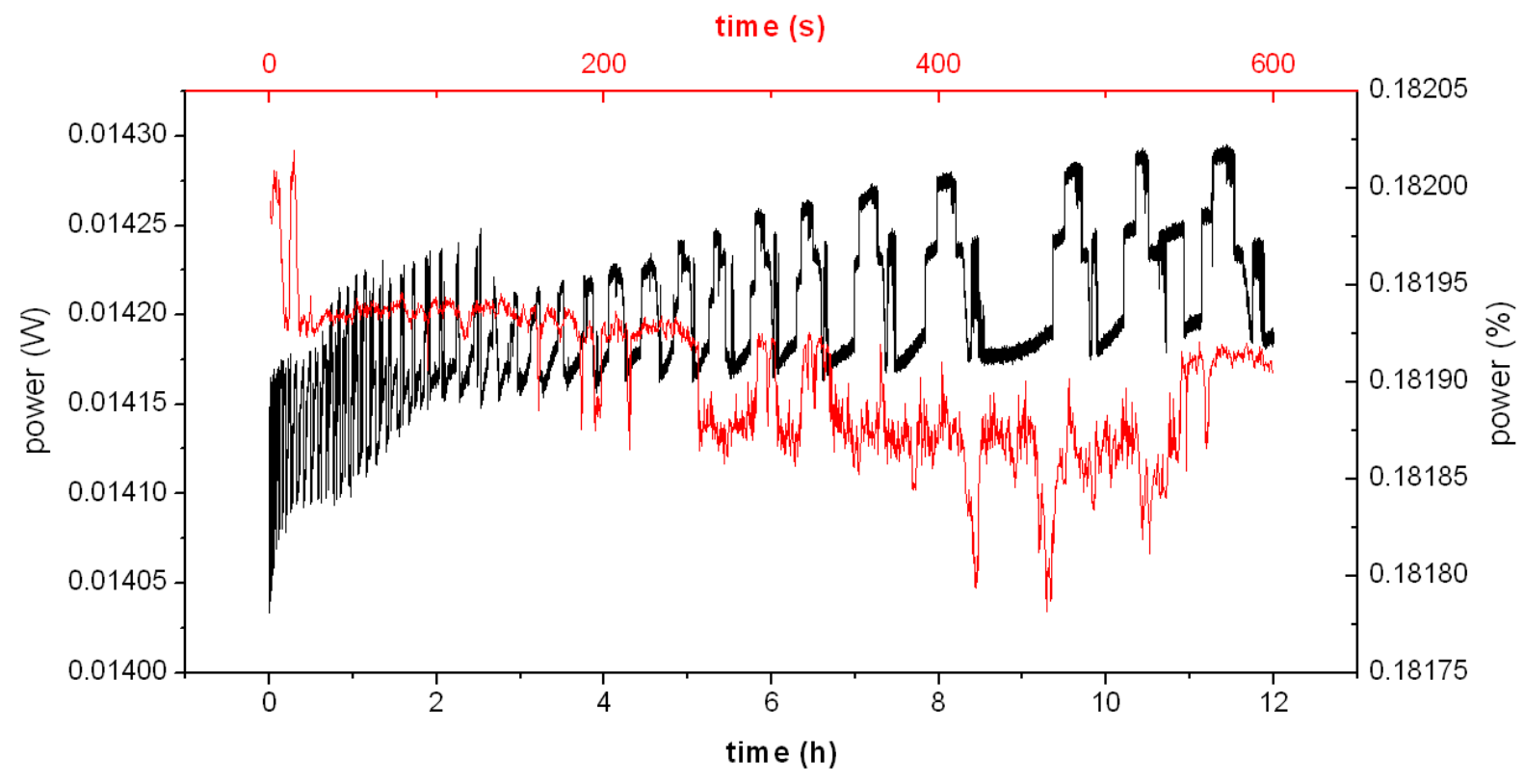

Fig. 2.18: Laser power drift at the sample over long (black line) and short (red line) time scales. Power measured with Thorlabs PM100D powermeter at a sampling interval of 300ms.

As an inherent feature, a laser diode is very sensitive to electronic noise. Whilst the mean output power might vary only a little, intensity fluctuations as well as pointing instabilities can occur at the $\mathrm{KHz}$ level and hence interfere with the actual measurements. These stability deviations were be seen by the naked eye and had proven to be independent from induced mechanical noise on the setup. Instead electronic noise induced form devices near by provided the pointing instabilities observed. By successive removal of different components, the on board USB connection of the driver was identified as the major source of noise.

Figure 2.19 shows the difference of pointing stability and intensity variations in high temporal resolution, monitored by the laser reflection signal on a glass slide. 
pointing instability spectrum

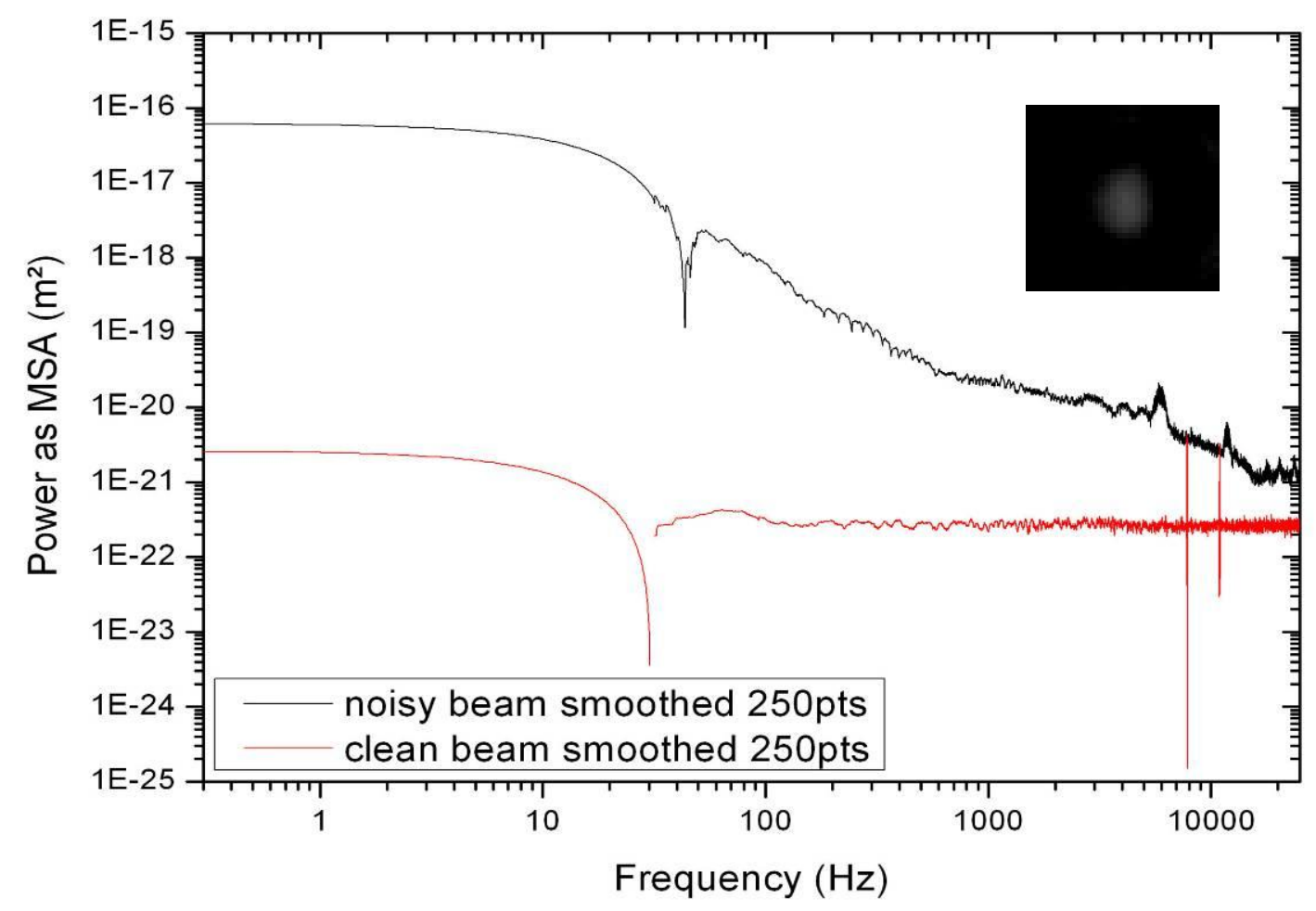

Fig. 2.19: Power spectra of lateral laser focus position deviation. The black line shows the frequency dependent position oscillations of the laser diode driven by the FL593 wavelength electronics board while the USB port is still connected, whilst the red line is the result of electrical isolation

Electrical isolation of the laser diodes, disconnection of the USB port whilst measurements and the addition of ferromagnetic dampers, efficiently reduces intensity fluctuations, but most importantly results in reduced pointing instabilities. A reduction of more than $90 \%$ in pointing instability from $70 \mathrm{~nm}^{2}$ down to $5.9 \mathrm{~nm}^{2}$ is observed. This value is well below the lowest standard deviation observed for particles in the strongest optical confinements of $10.5 \mathrm{~nm}^{2}$ at $250 \mathrm{~mA}$ laser diode driving current. The relative intensity fluctuations measured by comparison of the laser spot area deviation over time which is derived from a fixed greyscale threshold is also reduced by $80 \%$.

Whilst relaxation oscillations are mostly found in the high $\mathrm{GHz}$ region, shot noise and intrinsic intensity variations due to thermal effects are also mostly found at higher frequencies. The observed noise is well below these Frequencies and can be understood as internal power supply and remaining environmental noise. 


\section{THE OBJECTIVE}

The optical trapping objective should have a minimal numerical aperture $N A$ of at least 1.2 (25) which equals a half opening angle of $\theta=56^{\circ}$ given by

$$
N A=n \sin \theta
$$

with $n$ as the refractive index of the medium e.g. water $=1.33$. Apart from the numerical aperture, the objective needs to be as transmissive and achromatic as possible for the infrared light of the trapping laser. Based on measurements of Steven Block (28), the highest transmissivity was found for Zeiss Neofluar objectives, at wavelengths close to $808 \mathrm{~nm}$. Therefore we chose a Zeiss EpiPlan Neofluar 100x/1.3 oil objective for the optical trap.

\begin{tabular}{|c|c|c|c|c|c|c|c|}
\hline \multirow[b]{2}{*}{ Part no. } & \multirow[b]{2}{*}{ Manufacturer } & \multirow{2}{*}{$\begin{array}{l}\text { Magnification/tube length } \\
(\mathrm{mm}) / \text { numerical aperture }\end{array}$} & \multirow[b]{2}{*}{ Type designation } & \multicolumn{4}{|c|}{ Transmission $( \pm 5 \%)$} \\
\hline & & & & $830 \mathrm{~nm}$ & $850 \mathrm{~nm}$ & $990 \mathrm{~nm}$ & $1064 \mathrm{~nm}$ \\
\hline 461832 & Zeiss & $63 / 160 / 1.2$ water & Plan NeoFluar & 66 & 65 & 64 & 64 \\
\hline 506038 & Leica & $100 / \infty / 1.4-0.7$ oil & Plan Apo & 58 & 56 & 54 & 53 \\
\hline 85020 & Nikon & $60 / 160 / 1.4$ oil & Plan Apo & 54 & 51 & 17 & 40 \\
\hline 93108 & Nikon & $60 / \infty / 1.4$ oil & Plan Apo CFI & 59 & 54 & 13 & 39 \\
\hline 93110 & Nikon & $100 / \infty / 1.4$ oil & Plan Apo CFI & 50 & 47 & 35 & 32 \\
\hline 93110IR & Nikon & $100 / \infty / 1.4$ oil & Plan Apo IR CFI & 61 & 60 & 59 & 59 \\
\hline 93144 & Nikon & $100 / \infty / 1.3$ oil & Plan Fluor CFI & 67 & 68 & - & 61 \\
\hline
\end{tabular}
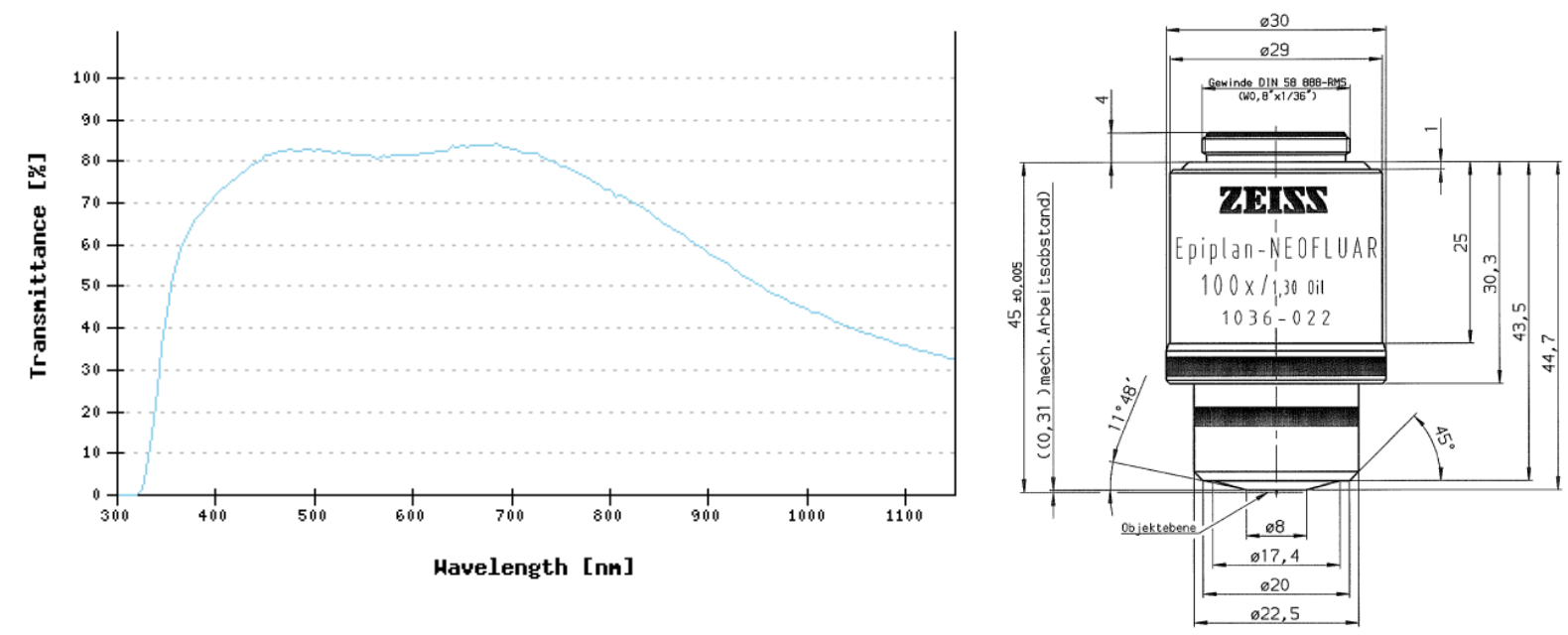

Fig. 2.20: Table: Transmissivity values obtained for different objectives at different wavelengths by S. Block et al. Graph: Transmissivity of Zeiss Epiplan-Neofluar 100x/1.3 oil objective at different wavelengths (provided by Zeiss). Sketch: Sketch of Zeiss Epiplan-Neofluar 100x/1.3 oil objective dimensions.

Using a 100x/1.3 oil objective in combination with an $\lambda=808 \mathrm{~nm}$ laser diode we can calculate an expected beamwaist radius by

$$
\omega=\frac{\lambda}{\pi \cdot N A}
$$

With an numerical aperture of NA $=1.3$ the theoretical beamwaist diameter is $\omega=396 \mathrm{~nm}$. 


\section{MICROSCOPE SETUP}

The microscope has been designed to be most rigidity in terms of mechanical oscillations and reliability of adjustment. The dimensions of the microscope have been kept as small as possible, so that it can also be integrated into a incubation chamber with controlled environmental conditions for living cell culture experiments.

The framework is based on an aluminium thread-bore plate and consists of Linos Mikrobank components. These are high precision aluminium cubes and plates to carry optical compounds which can be perpendicularly connected via hardened steal rods. These components are both very rigid and precisely manufactured and they allow for pre aligned rectangular assembly. The components used in construction of the microscope are given in figure 2.21.

\section{Optical trapping fluorescence microscope}

2 lens collimator
Objective Zeiss
HC 680/SP
Dichroidic mirror
BS 447/LP

Fig. 2.21: Sketch of the optical trapping fluorescence microscope. Components are indicated by color coded description 


\section{Optical trapping fluorescence microscope}

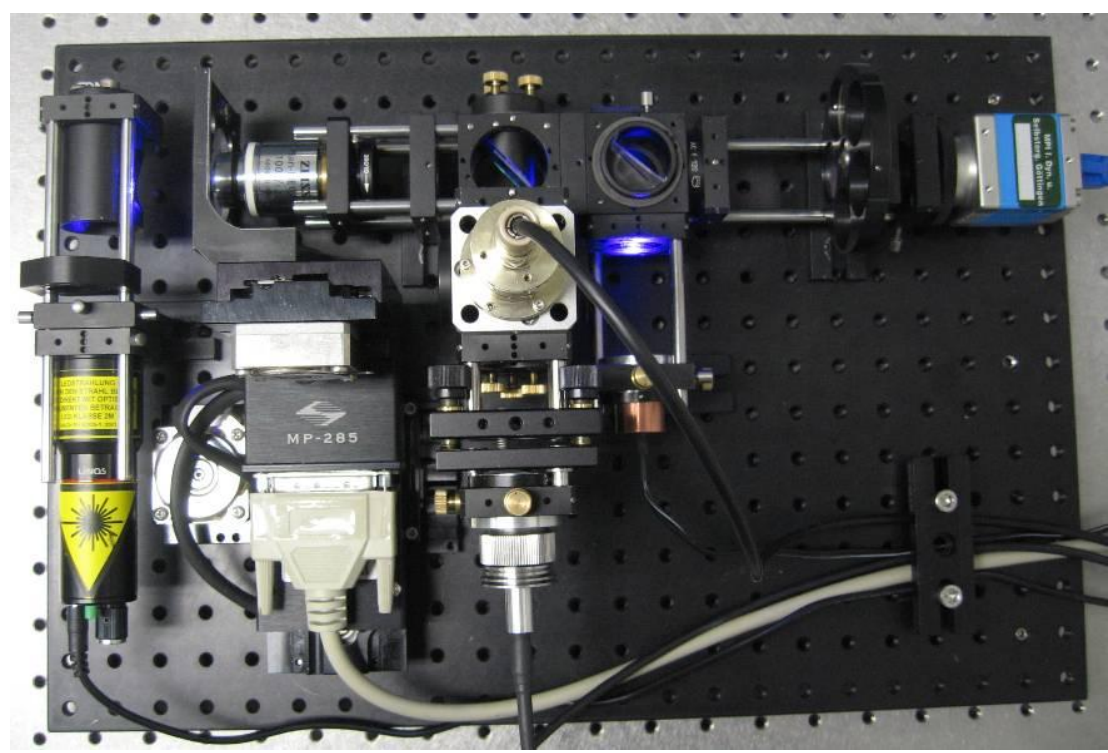

Fig. 2.22: Image of optical trapping fluorescence microscope setup. Note the a PCO pixelfly qe camera is mounted instead of the Phantom Miro described in figure 2.21.

\section{ILLUMINATION}

BRIGHTFIELD ILLUMINATION of the sample is done with two lens condenser, focusing the light of a high power white light CREE LED onto the sample. The light output of modern LEDs has increased to several hundred lumens for commercial single emitter chips with a size smaller than $A_{\text {Emitter }}<4 \mathrm{~mm}^{2}$. The big advantage of LED light sources, compared to ordinary light sources, is not only its efficiency and even spectrum, but also the possibility of very short on / off switching times, allowing for stroboscopic or pulsed illumination at $\mathrm{MHz}$ frequencies. The spectrum and output power of several available LEDs are compared against a XBO $75 \mathrm{~W}$ Xenon lamp in figure 2.23.

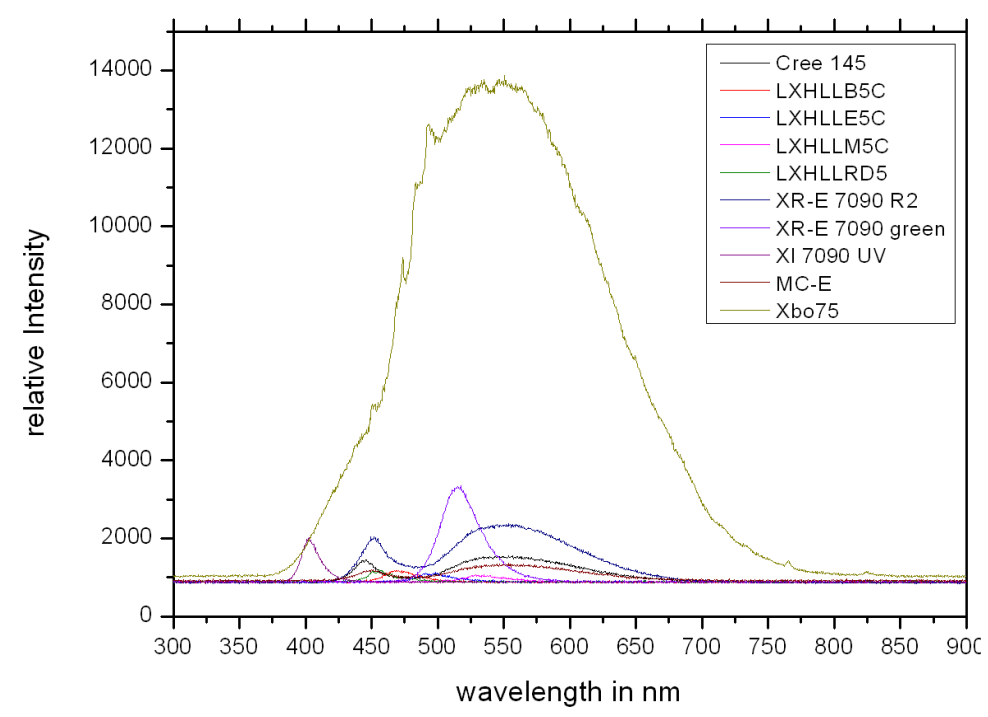

\section{LED comparison}

Fig. 2.23: Comparison of spectra and relative power of several lightsources as stated in the figure legend 
The different light sources, compared in figure 2.23, were coupled into a Olympus BX 61 microscope stand and focussed onto a Thorlabs PD 200 powermeter using an Olympus LMPlanFl 50x/0.5 objective.

Many different colours are currently available, however, the white light LED called Cree 145 shows a peak intensity of about $15 \%$ compared to a XBO 75 Watt Xenon burner at $\lambda=550$ $\mathrm{nm}$. The Cree 145 LED has been replaced by a XPGWHT-L1-1T-R5 CREE LED which is stated with $370 \mathrm{~lm}$ output compared to $145 \mathrm{~lm}$ and has been used for all experiments in this thesis, but could not be compared to it precursor directly.

\section{SUMMARY}

We developed an optical trapping fluorescence microscope on basis of Arthur Ashkins single beam gradient optical trap, described in 1983 (24). Basic technical considerations on optical trapping microscopy were discussed with Dr. André Beerlink prior to construction. The main technical features of the apparatus, that has been developed and constructed as part of this work, are summarized in the following list:

- Combined fluorescence and bright field microscope

- Independent dual beam optical trap

- Fluorescence sensitivity:

- Max. bright field frame rate:

- Max. Stroboscopic illumination rate:

- Max. dual beam trap switching frequency: $100 \mathrm{~Hz}$

- Min. theoretical lateral resolution:

- Min. Position resolution:

- Max. trapping force on $1 \mu \mathrm{m}$ ps beads: single molecule (TMV DNA, Sensicam) $120 \mathrm{kfps}$ at 1024 pixel (Phantom Miro 3)

$100 \mathrm{kHz}$

$400 \mathrm{~nm}$

$5 \mathrm{~nm}$

$65 \mathrm{pN} 2 \mathrm{x}$ 


\section{CALIBRATION OF THE OPTICAL TRAP}

Measuring forces is only possible in a well calibrated system. Especially when forces are very weak, all parameters need to be well known and noise has to be kept well below the signal threshold. Three different, but complementary methods of calibration have been used to calibrate the optical trapping setup. Each method will be described and results will be discussed within the following chapter.

\section{STOKES DRAG CALIBRATION}

The most direct way of measuring the stall force of an optical trap is to determine the escape velocity $v$ of a sphere of given radius $R$ in a fluid of given viscosity $\eta$, in order to calculate the Stokes drag $F$

$$
F=6 \pi \eta R v
$$

which is equal to the maximum trapping i.e. stall force. By either moving of the sample stage, or by inducing a flow within the fluid, the minimal velocity can be approached, at which the resulting drag force on the particle will overcome the maximum trapping strength at any given laser power. This method is easiest to access experimentally, because neither a high speed signal detection system, like a high speed camera or a quadrant photo diode, nor a submicron position calibration is needed to determine the velocity. Either the velocity of the stage is given or the relative velocity of the flowing medium can be determined by the streak length of the particles by a given exposure time of the camera and the length scales.

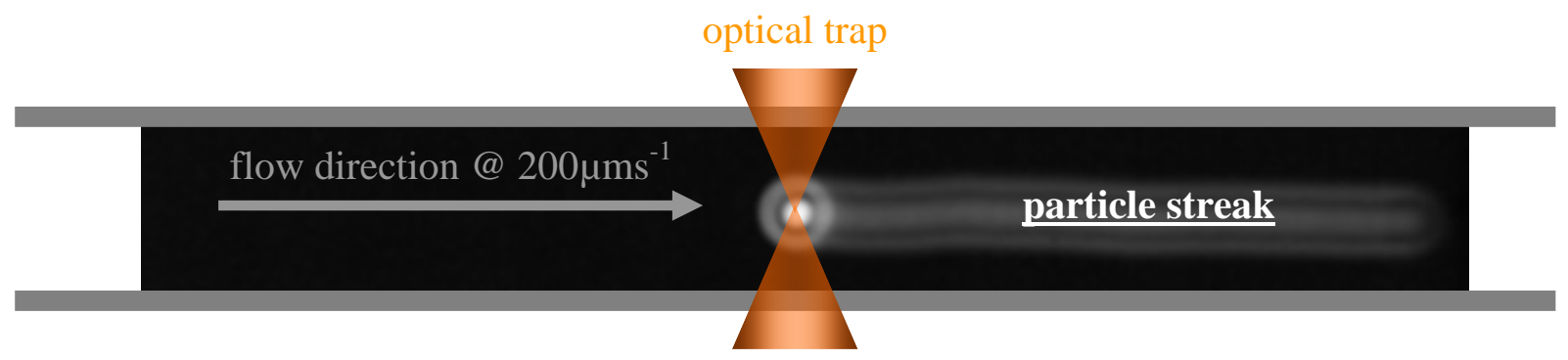

However, a source of error, often unseen without high speed imaging, is the stepping nature of many translation stages as well as oscillations within microfluidic flow. A tracer streak does not display the peak velocities in an oscillating flow, but only the average velocity. Therefore calculated stall forces in an optical trap are easily underestimated. 
In the following experiment flow properties have been analyzed in a microfluidic channel of $500 \mu \mathrm{m}$ in width and $50 \mu \mathrm{m}$ in height. Flow velocimetry has been performed with a $2 \mu \mathrm{m}$ polystyrene bead, optically trapped $5 \mu \mathrm{m}$ below the coverslip surface and released, so that every particle is traced in the focal plane as well as in the same position (centre of channel, $250 \mu \mathrm{m}$ away from the side walls) of the microfluidic channel.

\section{High speed particle tracking}
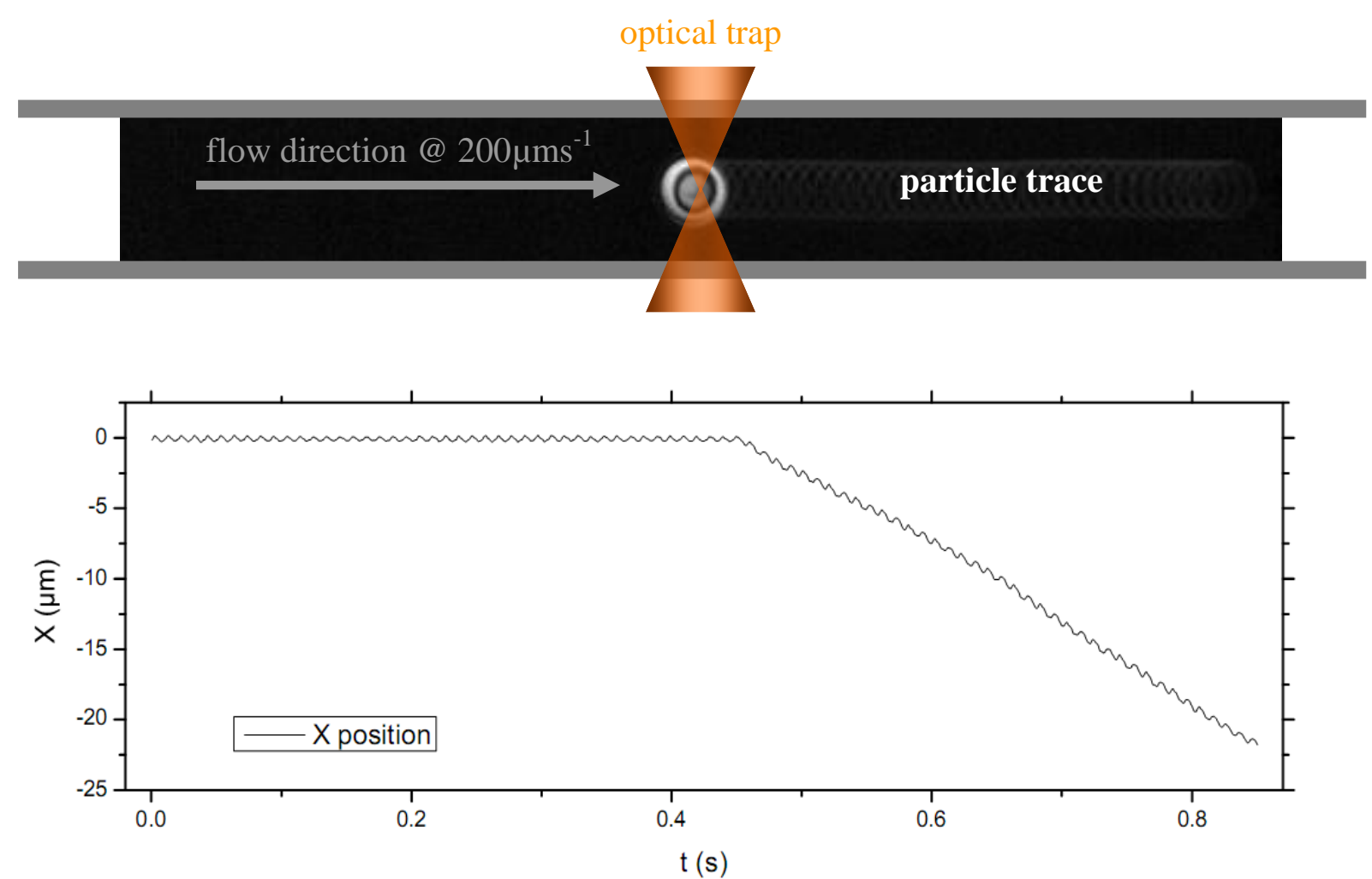

Fig. 2.24: $2 \mu \mathrm{m}$ polystyrene particle trace recorded at 2000 frames per second at a mean flow velocity of $55 \mu \mathrm{m} \cdot \mathrm{s}^{-1}$ with peaks up to $200 \mu \mathrm{m} \cdot \mathrm{s}^{-1}$. Laser diode current was set to $75 \mathrm{~mA}$, flow was driven by pump.

Oscillations in flow velocity are strongly dependent on the pumps and syringes used in the experiment. Possible sources of noise can be stepping motors, rough spindles or a loose gearbox for example. Moreover, syringes show prominent stick slip behaviour instead of sliding deviation. The static friction scales with the piston diameter, hence syringes with the smallest appropriate size should be chosen. To minimize oscillations in flow and consequently the error in calibration measurements, different available syringe pumps have been tested, using a $50 \mu 1$ Hamilton syringe with a piston diameter of only $1.030 \mathrm{~mm}$. Oscillations in flow velocity have been measured by analysis of the escaped particle traces. 
flow velocity oscillations

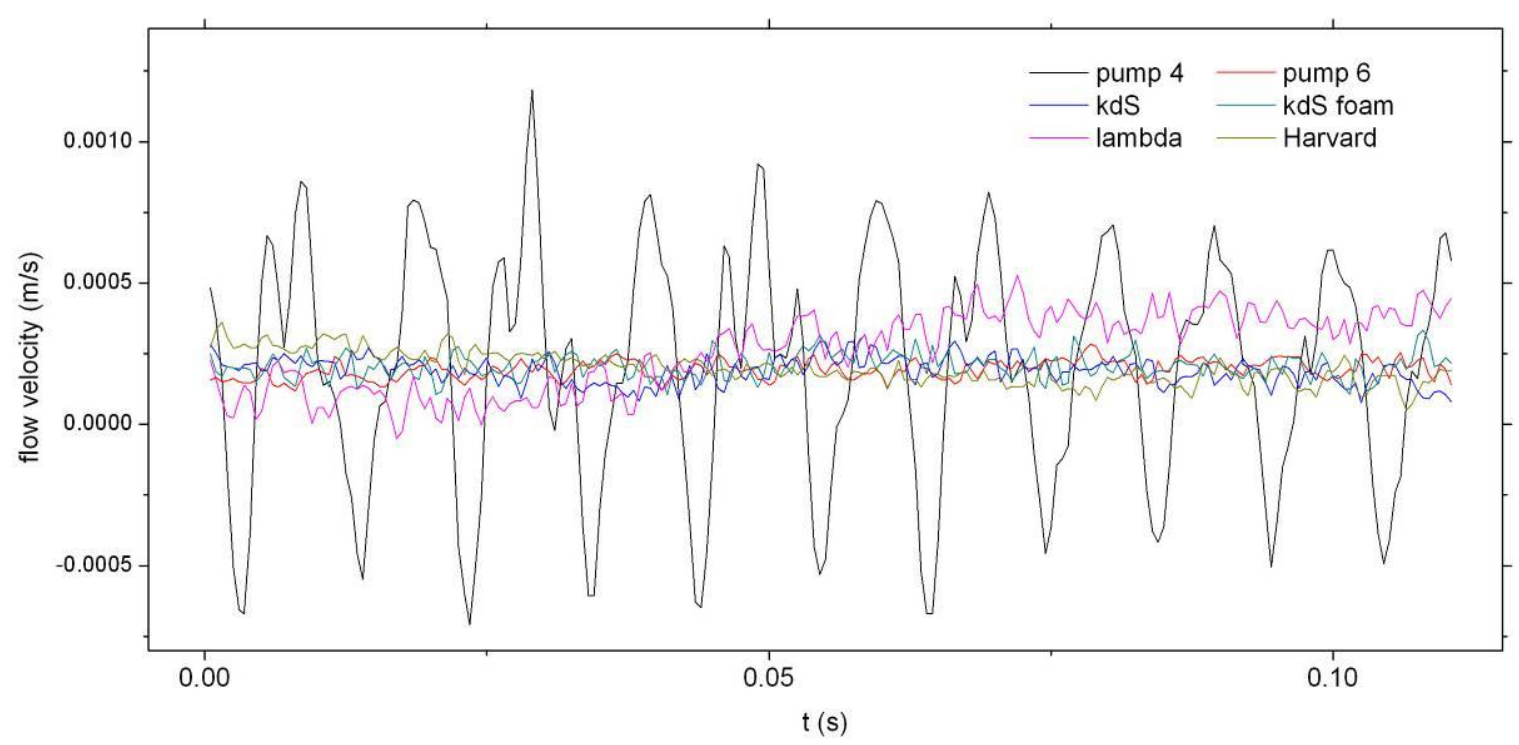

Fig. 2.25: Flow velocity oscillations in the particle escape traces driven by different pumps but all trapped beforehand at $75 \mathrm{~mA}$ diode current. Data is normalized to the fasted flow rate of pump 6.

Pump number 4 and 6 have been designed and built in house by Udo Kraft and Thomas Pfohl, where number 4 has been equipped with a reduction gear box driving the spindle and number 6 is directly driven by the stepping motor (Panasonic MUMS5AZA1E0S). Unfortunately all pumps show strong oscillation in the resulting flow velocity that are bigger than $16.5 \%$ at least (pump nr.6), going up to $200 \%$ (pump nr. 4) at flow rates of $2 \mathrm{~mm} \cdot \mathrm{s}^{-1}$. Damping of the piston slider using elastomers can reduce amplitudes as much as $10 \%$ and more, according to the damping material used. Unfortunately, damping the piston slider comes with the cost of increased response and relaxation times rendering their usage impractical for several experiments.

With respect to these results, all further experiments have been conducted with pump number 6 or the Harvard apparatus $\mathrm{PhD}$ ultra, including extra silicon damping of the piston slider for experiments that only require constant flow velocities.

Stokes drag calibration experiments were carried out with a Harvard apparatus $\mathrm{PhD}$ ultra pump, programmed to drive a very shallow linear pumping ramp, starting at $50 \mu 1 \cdot \mathrm{h}^{-1}$ going up to $400 \mu \mathrm{l} \cdot \mathrm{h}^{-1}$ volume flow within $120 \mathrm{~s}$. The laser power was changed in $25 \mathrm{~mA}$ steps, from the minimum laser power of $75 \mathrm{~mA}$, up to the maximum of $250 \mathrm{~mA}$. The event of particle escape, that is when the drag force overcomes the optical stall force, was recorded with $16 \mathrm{kfps}$ and $10 \mu \mathrm{s}$ exposure time. The particle velocity oscillations in flow and their distribution, fitted by a Gaussian distribution function to obtain the mean velocity and hence the Stokes drag of the particle, is shown in figure 2.26. 

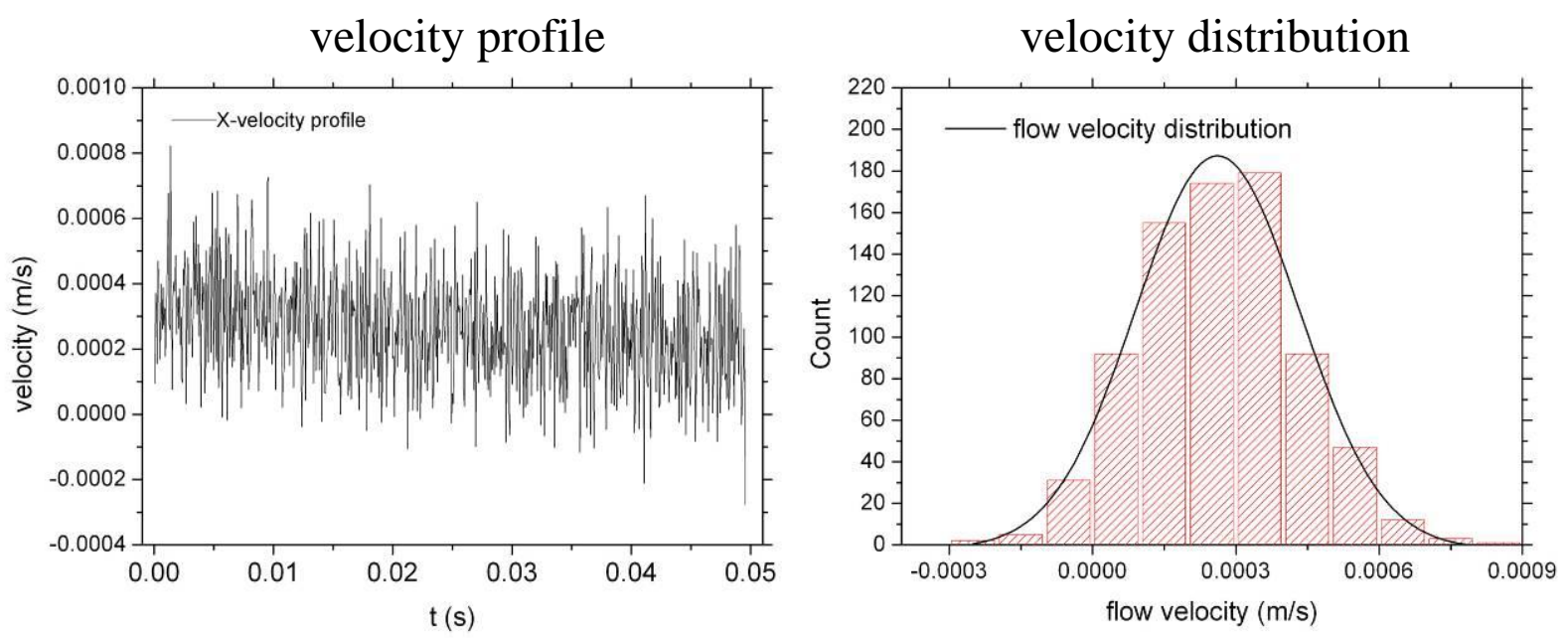

Fig. 2.26: The upper left figure shows the escape velocities in $\mathrm{x}$ direction of a $2 \mu \mathrm{m}$ polystyrene sphere after it had been trapped at $75 \mathrm{~mA}$ laser current. The upper right figure shows the distribution of $x$-velocity distribution, fitted by a Gaussian function (center = $2.6136 \cdot 10^{-4} \mathrm{~m} / \mathrm{s}$, width $=3.43 * 10^{-4} \mathrm{~m}$, sigma $=$ $\left.1.72 * 10^{-4} \mathrm{~m}\right)$. The lower right figure shows the $\mathrm{x}$-velocity distribution of a free diffusive $2 \mu \mathrm{m}$ polystyrene sphere in the very same experiment (center $=0 \mathrm{~m} / \mathrm{s}$, width $=1.73 \cdot 10^{-4} \mathrm{~m} / \mathrm{s}, \sigma=$

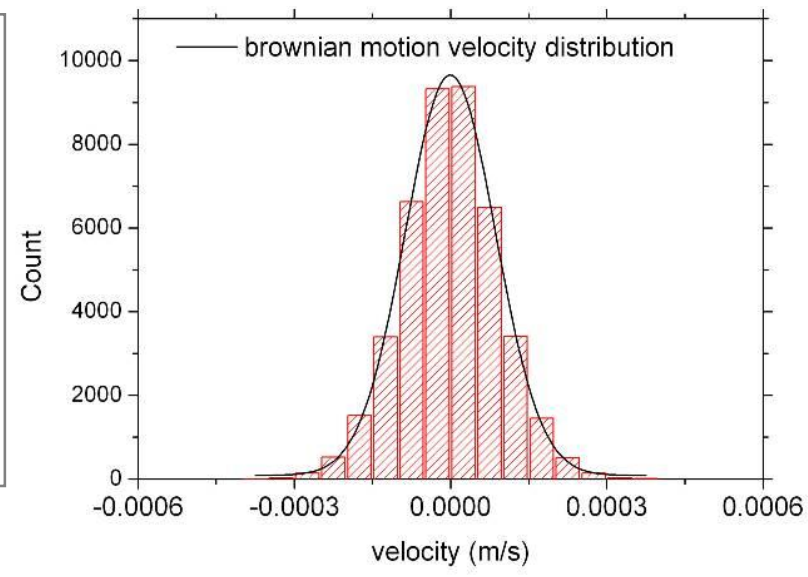

Brownian motion of a free diffusive $2 \mu \mathrm{m}$ polystyrene bead itself shows a velocity distribution in flow direction within the same order of magnitude $\left(\sigma=1.72 * 10^{-4} \mathrm{~m} / \mathrm{s}\right.$ compared to $\sigma=8.67 * 10^{-5} \mathrm{~m} / \mathrm{s}$ ) determining the lower limit of the oscillation amplitude.

The Stokes drag on the particle can now be calculated using the mean flow velocity value which can be obtained by measuring the streak of a particle (Fig. 2.8). This results in an underestimation of the actual value, because a particle will not escape the optical well, when the flow has reached a certain mean velocity but at the very first peak, where the drag force is stronger than the maximum optical stall force of the trap. The high speed imaging experiments reveal a difference between particle streak and particle trace measurements of about $15 \%$, even though very precise syringe pumps have been used. In consequence, using low speed imaging techniques unfortunately requires high precision instrumentation to minimize fluctuations in flow velocities and hence achieve precise measurements. In the end, we were not able to significantly reduce fluctuations in flow, but it was not possible to reduce them below detection thresholds. 


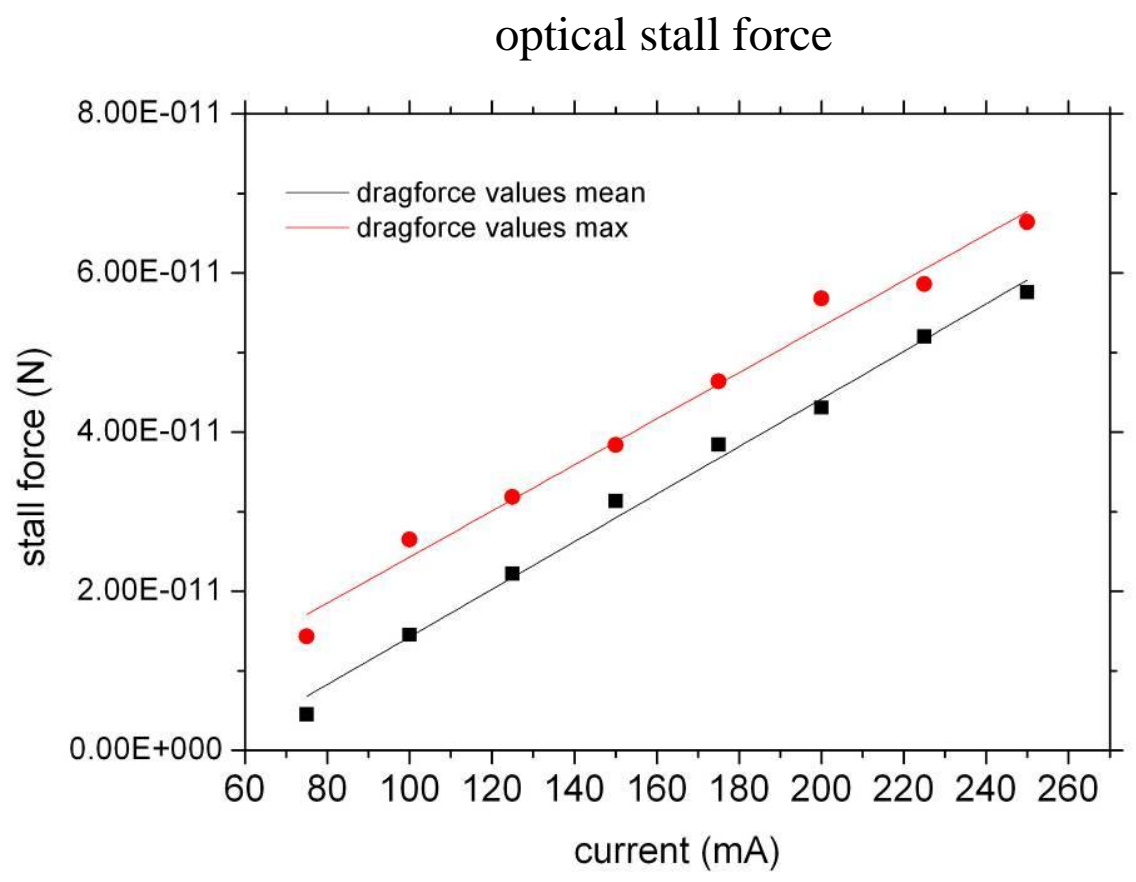

Fig. 2.27: Optical stall force of the VPSL 808-200 sm laser diode within the SuK collimator over driving current. The black line is a linear fit to the mean drag force data. The red line is a linear fit to the maximum drag forces measured at each laser current.

\section{FLIP-FLOP CALIBRATION}

The setup includes two independent optical traps with crossed polarization orientations. In combination with a high speed imaging system for direct video calibration instead of a quadrant photodiode, it is possible to analyse both complex shaped particles in optical confinement as well as multiple particles at the same time.

A technique for rapid calibration for stall forcesin both of both $x$ - and $y$-direction via video microscopy has been utilized by W. Singer et al. (무). Inertia of a $2 \mu \mathrm{m}$ polystyrene bead, accelerated at a rate of $2.5 \mathrm{~m} \cdot \mathrm{s}^{-2}$ at $15 \mathrm{~mW}$ laser power is as low as $F_{I}=10 \mathrm{fN}$ and can be ignored, compared to hydrodynamic friction forces of several pico-Newton in this regime. The velocity of a particle which is being attracted by an optical trap, can be directly used to calculate the drag force acting on it. Using two optical traps in close proximity and in alternating fashion now, will cause a particle to flip from one trap to the other, with different velocities at different positions. Instead of recording the particle as it is dragged out of the trap when the maximum stall force is overcome, using this method, the particle is traced from the very onset of attraction. The traces describe not only the center part, but also the tails of the optical potential well, which are elsewise inaccessible. 
Several technical advantages arise from this technique, e.g. the very same particle is used over the whole calibration procedure and deviations in diameter or shape of the calibration particle cease to apply. The same is true for deviations in cover slip distances due to inaccuracy of the translation stage, because the sample position does not need to be changed at all. Moreover, no external flow has to be induced by some extensive fluid flow or sample translation setup with limited precision, as discussed before. Another advantage of this "flip flop" calibration method educes form its repeatability: with a trapping time of $5 \mathrm{~ms}$, one can get 200 data points in only one second, quantitatively increasing precision of the measurement.

\section{dual beam optical trap}

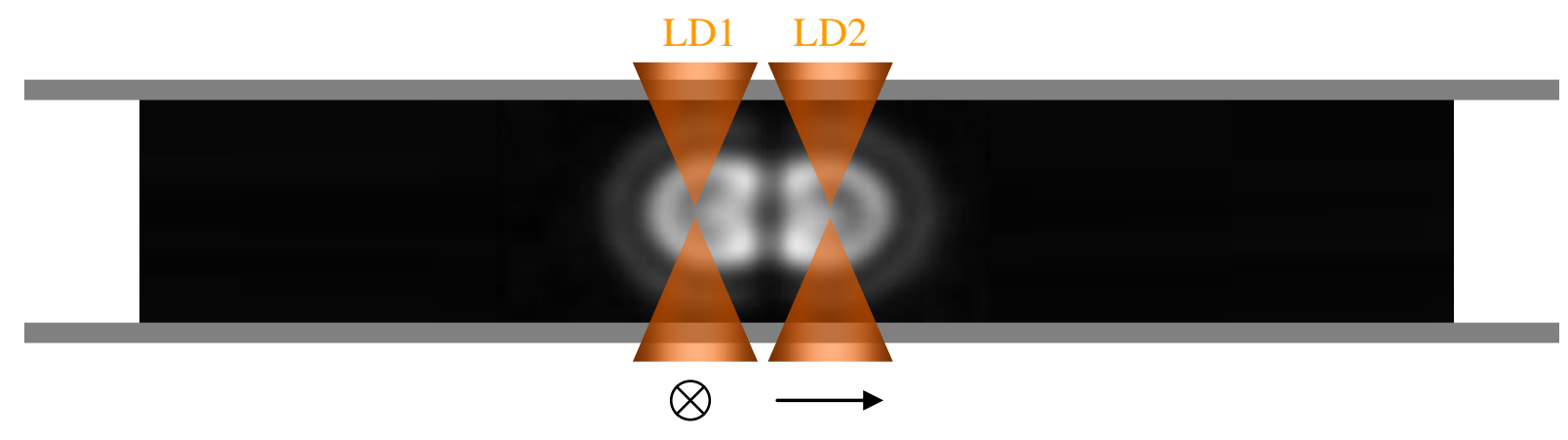

Fig. 2.28: Setup of the dual beam optical trap for the flip-flop measurements.

The experiment has been conducted in a stop flow microfluidic chamber of $50 \mu \mathrm{m}$ in height, where a $1.025 \mu \mathrm{m}$ polystyrene bead has been trapped $5 \mu \mathrm{m}$ below the coverslip surface, at a maximum possible distance of one particle diameter. Both VPSL 808-200 sm laser diodes beams are combined via a polarizing beamsplitter cube. Their polarization orientation is perpendicular and no interference between the two laser beams takes place. The two lasers are powered by a dual laser driver FL593 from Wavelength Electronics that allows for independent TTL control for each laser diode. The maximum analogue modulation frequency of this device is given by $500 \mathrm{kHz}$, whilst laser diodes can be modulated at $\mathrm{MHz}$ frequencies. Analytically, the maximum fall and rise times are about $1 \mu \mathrm{s}$ and can be ignored, given the observation timescales. In this experiment, the lasers were alternately turned on and off, at a frequency of $10 \mathrm{~Hz}$ and the bead displacement was recorded at $25 \mathrm{kfps}$ with $10 \mu$ s exposure time. 
flip flop particle trace

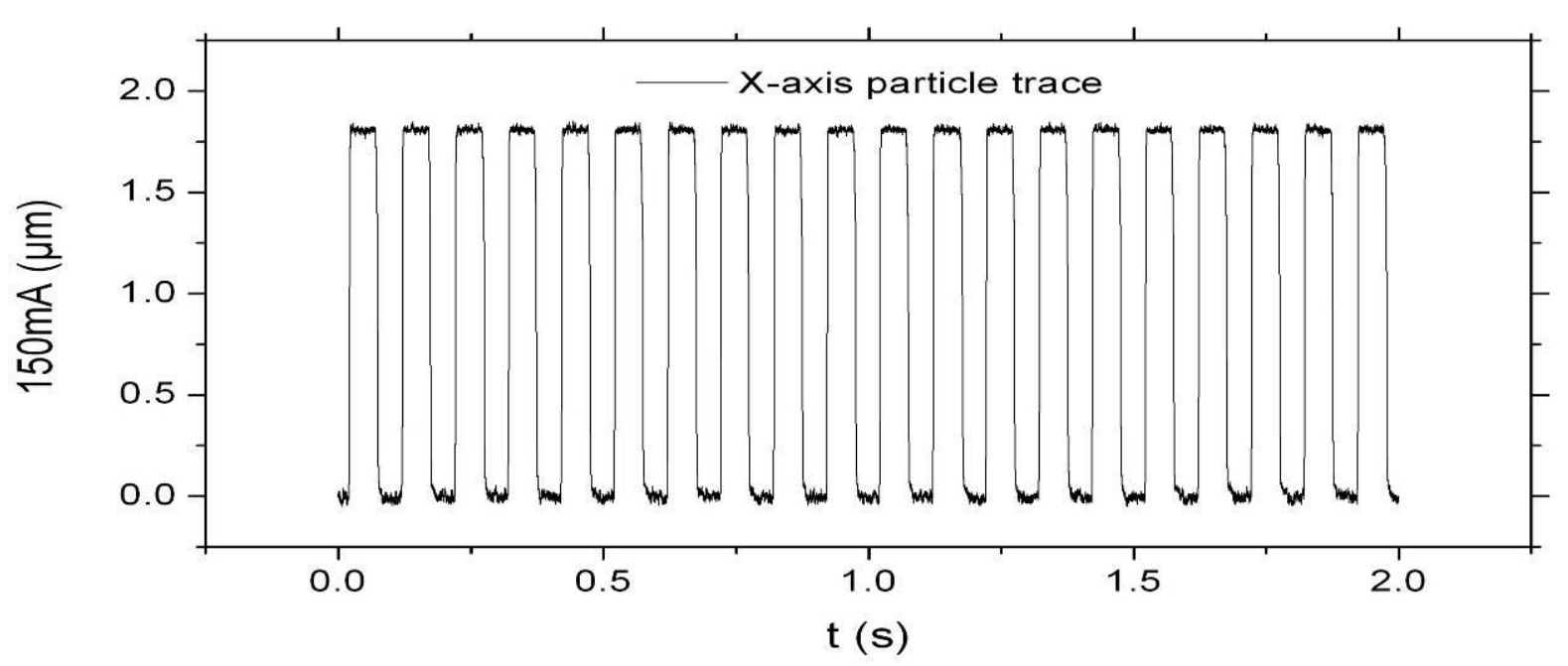

Fig. 2.29: Particle trace in $X$-axis direction over time. The bead has been alternately trapped between two separate optical traps with a frequency of $10 \mathrm{~Hz}$ and a laser power of $150 \mathrm{~mA}$

The flip-flop particle trace gives a quantitative measure of the attractive force over the distance towards the center. All particle traces are overlaid and the mean position over time is calculated to derive the velocity and force profile over position.

\section{flip flop particle trace zoom}
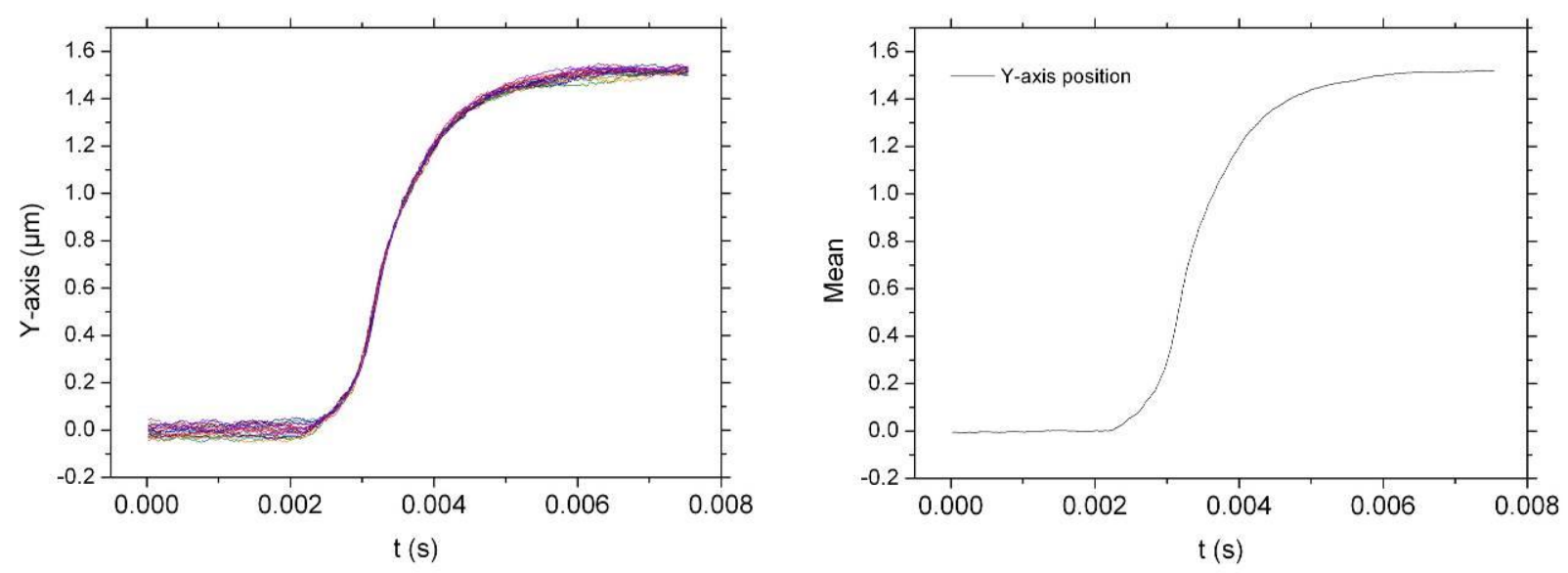

Fig. 2.30: Left: Particle traces in $Y$-axis direction over time for several flip-flop events. Right: Mean Particle trace in $Y$ axis direction over time.

The two optical traps are of different design, whilst the reference trap has been assembled, collimated and characterized by Schäfter \& Kirchhoff company, the steering beam is adjustable not only in $X Y$ directions but also in $Z$ direction during the experiment. 
The following measurements have been conducted with the steering beam collimated and hence focussed in the focal plane of the microscope objective, determined by the focal plane of the pre-adjusted S\&K laser. In figure 2.31 the velocity and hence the Stokes drag which is derived from the particle trace, is plotted over the position relative to the trapping center.

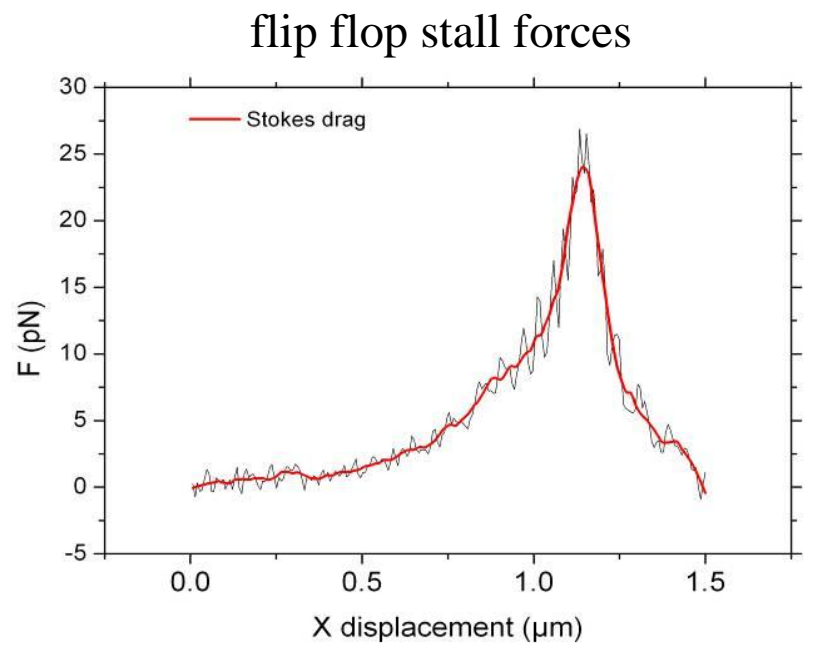

Fig. 2.31: Stokes drag derived from particle trace vs. position relative to the trapping center.

All four axes of both lasers have been quantitatively analyzed over the complete power range of the light source, utilizing this method for rapid yet precise calibration, comparison, and verification of the usually carried out Stokes drag calibration. The maximum attractive stall forces of both traps over laser power are shown in figure 2.32 .

Flip-flop stall force calibration

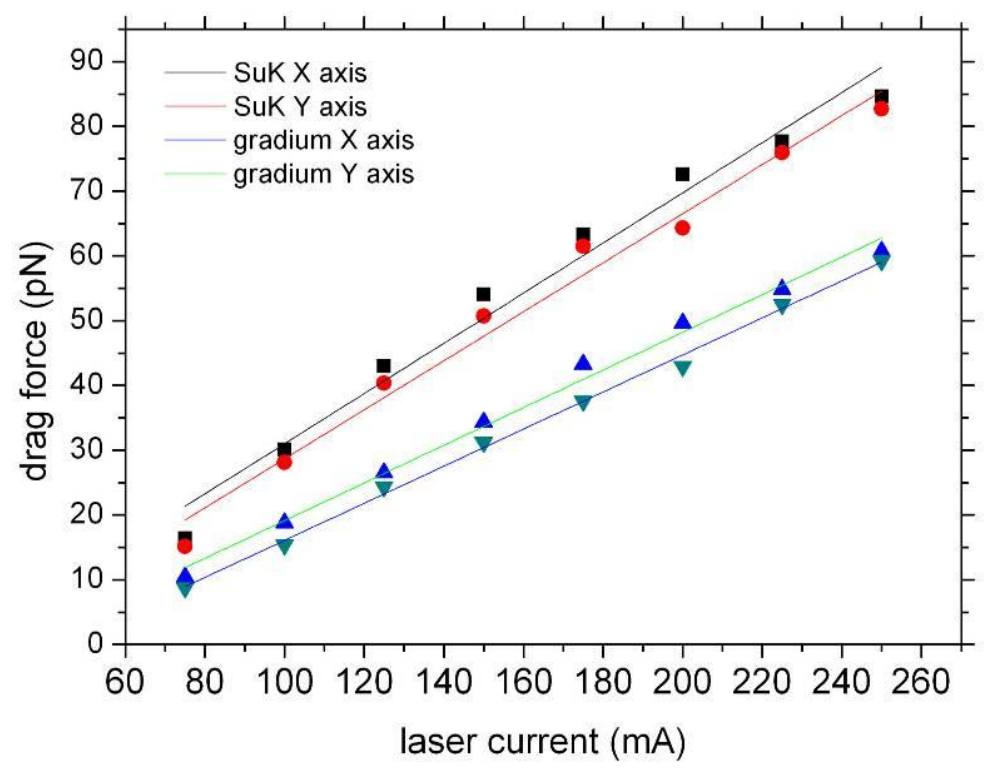

Fig. 2.32: Maximum attractive stall forces of both traps vs. laser power. 
The linear dependence of the trapping force over laser power can be verified with a remaining standard error factor of 0.02 . Both axes of each laser have the same slope varying by a factor of only 0.003 indicating a very precise con-axiality of both beams with the optical axis of the objective. The optical forces on dielectric particles are dependent on the polarization of the laser beam which can be seen by the different offsets of the stall force of crossed polarisation orientations. A comparison of both calibration results, obtained either by maximum stall force or by measuring the maximum attractive force by the flip-flop method is shown in figure 2.33 .

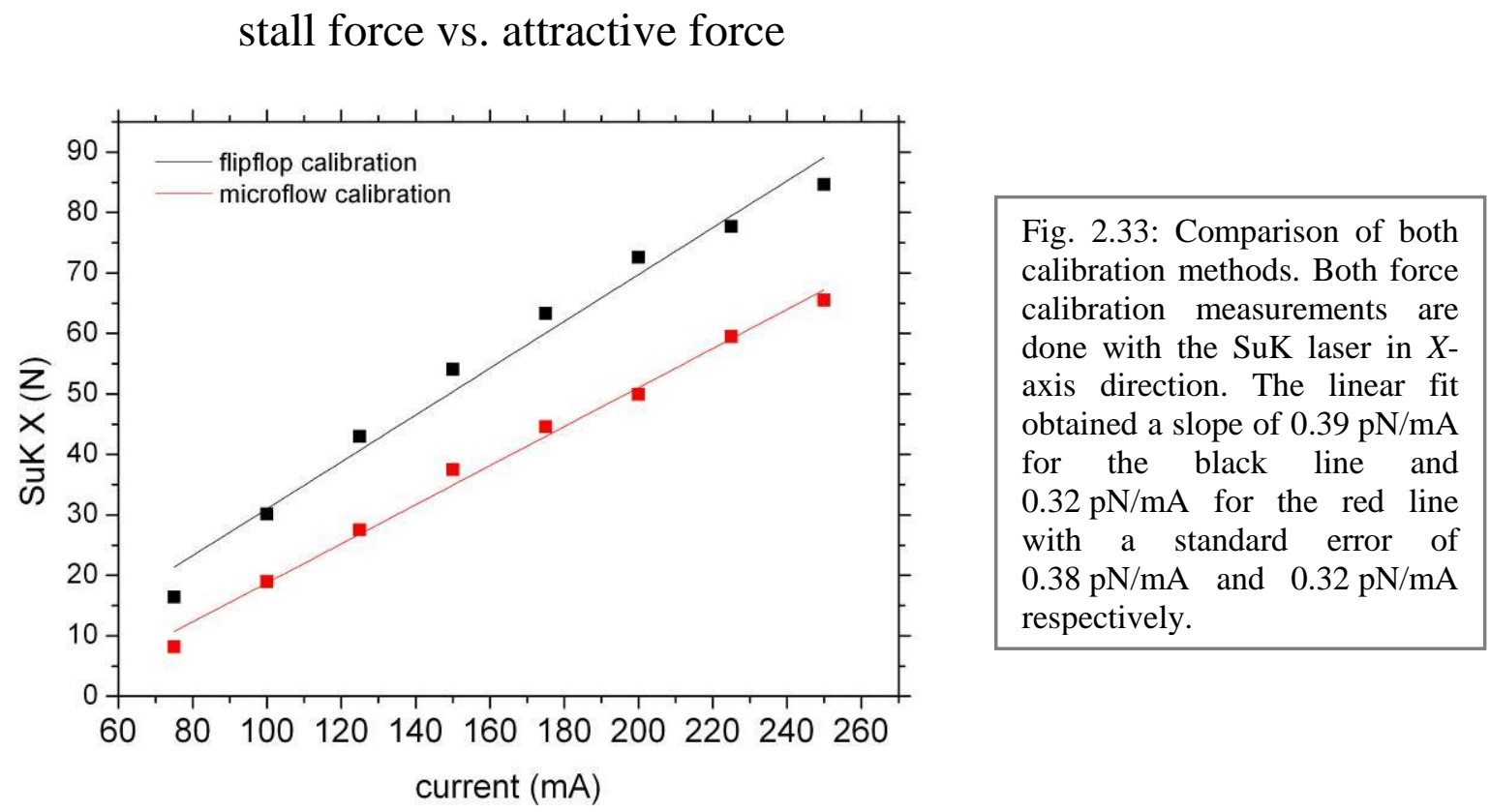

The comparison of the stall force with the attractive force calibration method reveals an underestimation in optical stall force measurements using standard escape velocity methods as described in 2.24. These findings indicate a general underestimation of trapping forces by the latter method which may be due to oscillations of the induced fluid flow. 


\section{OSCILLATION CALIBRATION}

The stiffness of an optical trap can be measured in two different ways. The optical potential in lateral direction can be compared to a spring using the Hookian spring model, where the force is measured by displacement. With

$$
F=-k x
$$

where $F$ is the force acting on the spring, $x$ is the deflection of the spring and $k$ gives the stiffness. Using

$$
F=\frac{k_{B} T}{\langle s\rangle}
$$

where $k_{B}$ is the Boltzmann constant, $T$ the temperature of the system and $\langle s\rangle$ is the variance of particle position in the trap.

\section{POSITION DISTRIBUTION CALIBRATION}

The particle position distribution over time can be plotted as a histogram. In figure 2.34, the particle position histogram of a $2 \mu \mathrm{m}$ polystyrene sphere, trapped at $10 \mathrm{~mW}$ laser power in water, fitted with a Gaussian function, is shown. The particle position distribution over laser power are plotted in figure 2.35. With increasing laser power, the particle is being confined to a smaller and smaller volume, as the trap stiffness increases.

\section{particle positon distributions}

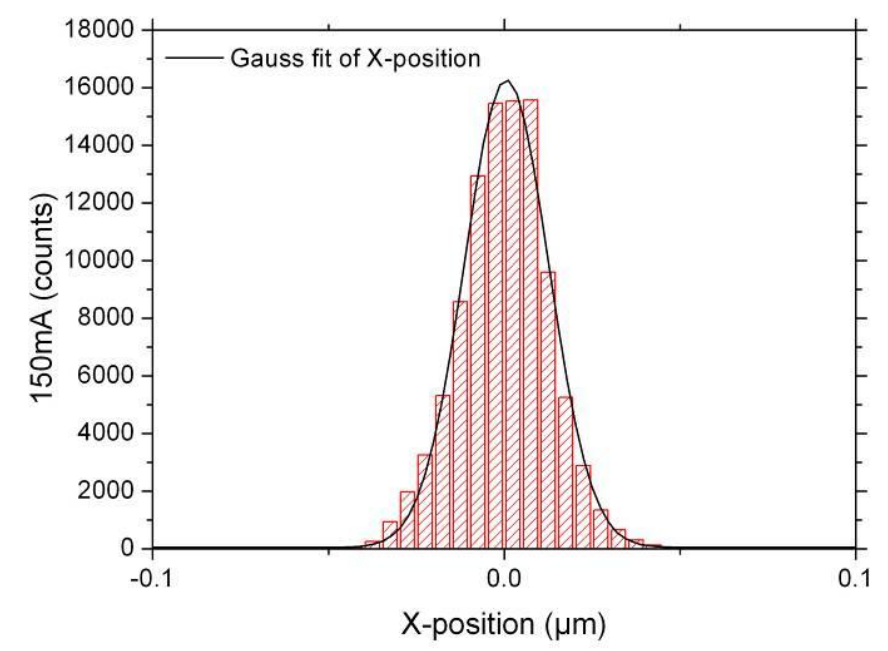

Fig. 2.34: Particle position histogram of a $2 \mu \mathrm{m}$ polystyrene sphere trapped at $10 \mathrm{~mW}$ laser power in water. The Gaussian fit is shown in black. 
The upper plot shows the Gaussian fits of the particle position histogrmas over laser power. With increasing power, the particle is being confined to a smaller and smaller volume as the trap stiffness increases.

\section{optical confinement}

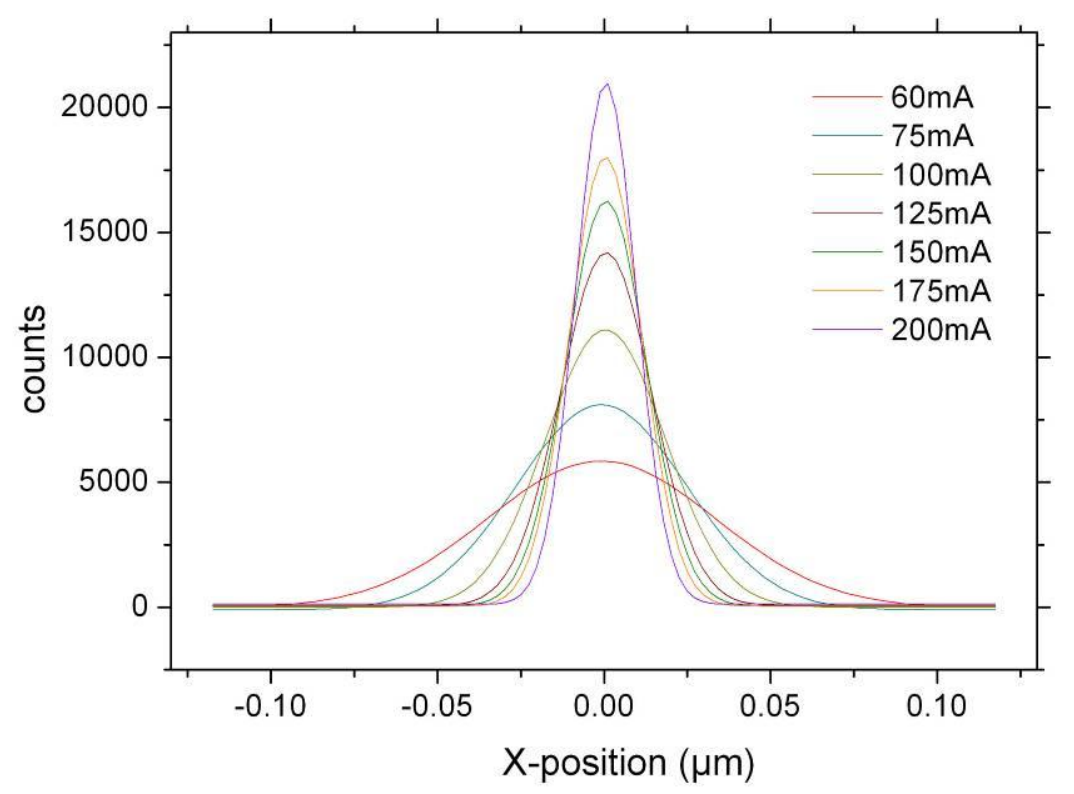

Fig. 2.35: Gaussian fits of particle position distribution at varying laser

The trap stiffness calculated from the particle position distributions (Fig. 2.36) shows an almost linear dependence on the laser current, but at higher laser currents an increased scattering of the data is observed.

Trap stiffness by variance

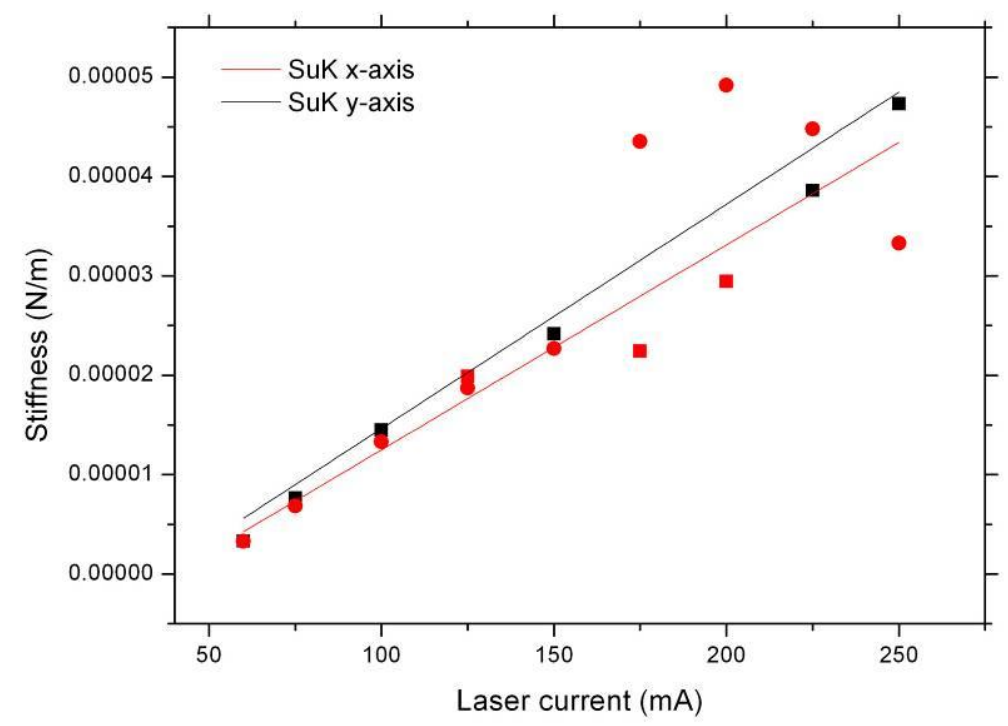

Fig. 2.36: Calculated stiffness from the particle position distributions vs. laser current. 


\section{POWER-SPECTRUM CALIBRATION}

The power-spectrum of a $2 \mu \mathrm{m}$ polystyrene bead trapped at $150 \mathrm{~mA}$ laser current is shown in figure 2.37. From the power spectra at different laser currents (fits of the power spectra vs. laser current are shown in figure 2.38), the trap stiffness for $x, y$ and $z$-direction can be calculated (Fig. 2.39).

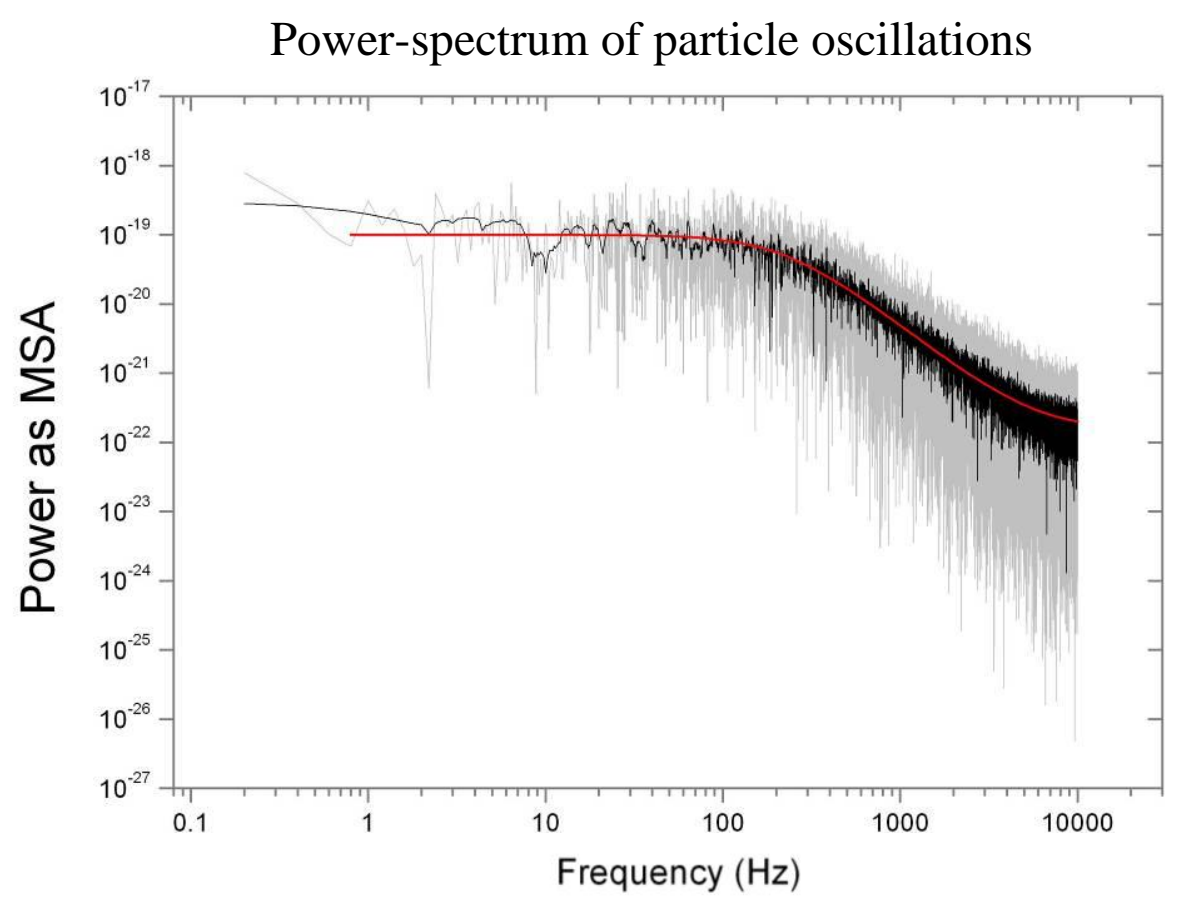

Fig. 2.37: Power spectrum of a $2 \mu \mathrm{m}$ polystyrene sphere trapped at $150 \mathrm{~mA}$ laser

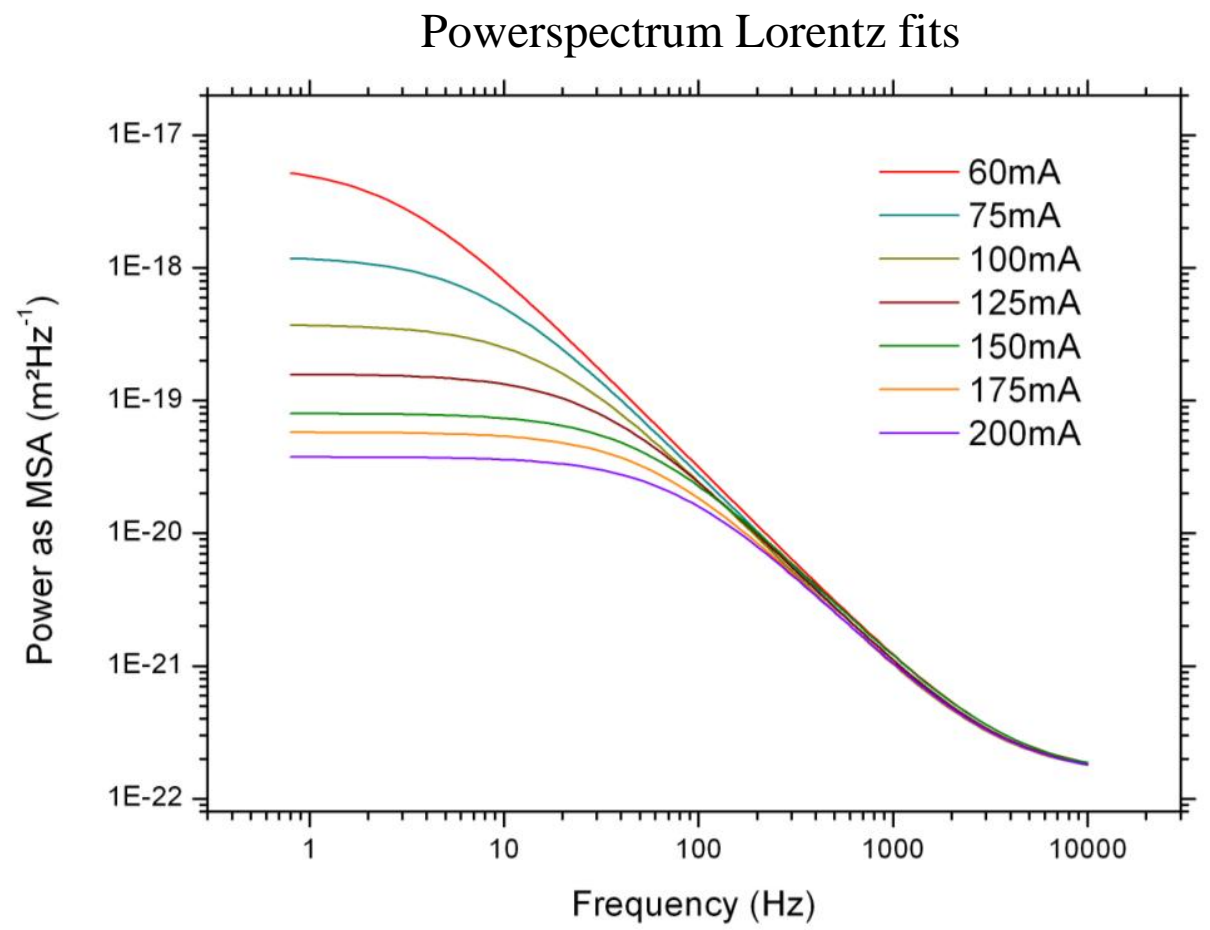

Fig. 2.38: Power spectra of a $2 \mu \mathrm{m}$ polystyrene sphere trapped in water vs. laser 
trap stiffness by power-spectrum

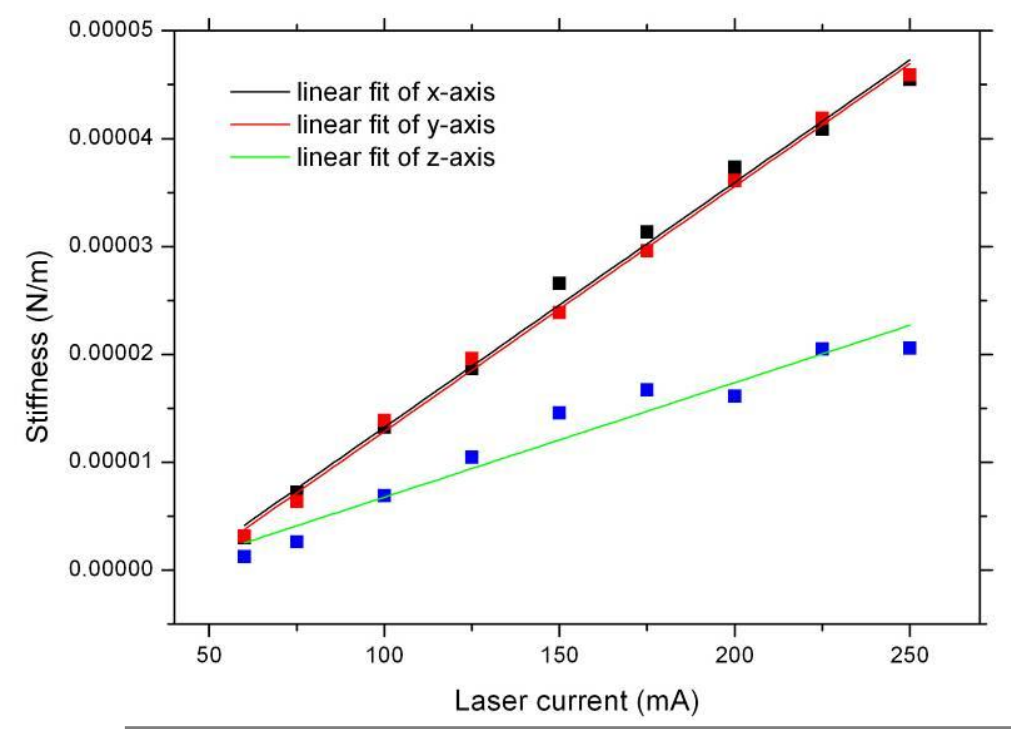

Fig. 2.39: Trap stiffness of $x, y$ and $z$-axis vs. laser current.

The trap stiffness of the $x$ and $y$-axis has the same linear increase with laser current. As expected, the absolute stiffness and the slope of the z-axis stiffness is weaker. Comparing the trap stiffness, calculated from the power-spectra, with the calculated stiffness from the particle position distributions, show a nice agreement (Fig. 2.40). However the results from the power-spectra analysis lead to results with smaller errors.

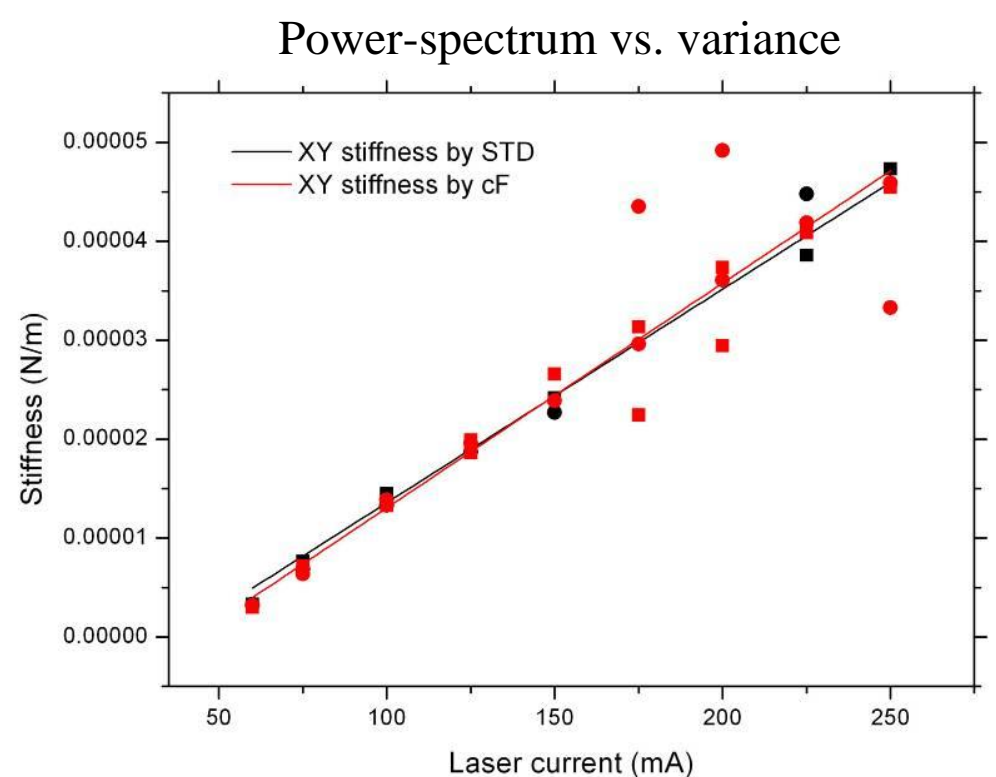

Fig. 2.40: Comparison of the trap stiffness calculated from power-spectra with the calculated stiffness from the particle position distributions.

In summary we have successfully constructed a high performance optical tapping microscope, using high speed video microscopy methods and thus allowing for simultaneous analysis of multiple traps as well as complex shaped particles like living trypanosomes. 


\section{IMAGING}

In order to analyze particle movements in optical traps, fluid velocities in microchannels and motility of trypanosomes with high accuracy as well as quantity, it is necessary to derive the data in an automated fashion. In video microscopy, this is done by virtual image manipulation, using specialized algorithms to calculate image properties also called image processing (31).

\section{COMPUTER VISION}

Image processing describes the conversion of image information into numbers. When we see a moving trypanosome for example, the computer only sees a number of pixels with different grey values or colours, changing from image to image. The two main aspects of image processing on image stacks can therefore be described as:

\section{Detecting the object or feature of interest}

2. Determining its changes from image to image

The two main challenges in doing so are the changing parameters of the background as well as of the object of interest itself. Only the difference between these parameters describes the information to be extracted, hence image processing is, to a huge extend, about defining parameters.

\section{IMAGE PARAMETERS}

The information of an image is given by the distribution of pixel values, for if we see a trypanosome in an image, this distribution is obviously different from the background. In the following procedures, the information content has always been categorized and derived by three main aspects:

1. Pixel value (the grey value of the object is defined by a certain threshold)

2. Pixel area (the size of the object is defined by a certain threshold)

3. Pixel displacement (the movement over time is defined by a certain treshhold)

In other words, an object of a certain brightness and size which can not move faster than a certain velocity, is distinguished from the background noise. 


\section{IMAGE PROCESSING}

The basic image processing routines that are equal to all particle tracking and trypanosome velocimetry measurements are described in the following protocol. All image processing is done using FIJI based on the open source NIH-ImageJ software (http://rsbweb.nih.gov/ij) including cited plug-ins. The theoretical background of the applied imaging methods is given by Wilhelm Burger and Mark J. Burge in "Digital Image Processing with Java and ImageJ".

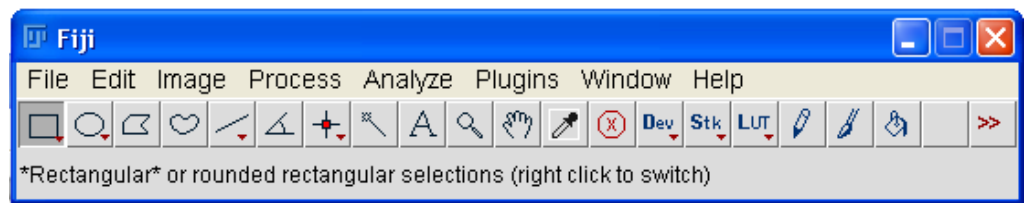

1. Create artificial background image by averaging all frames of the original image

- Image

- Stack

- Z-Project

- Average Intensity
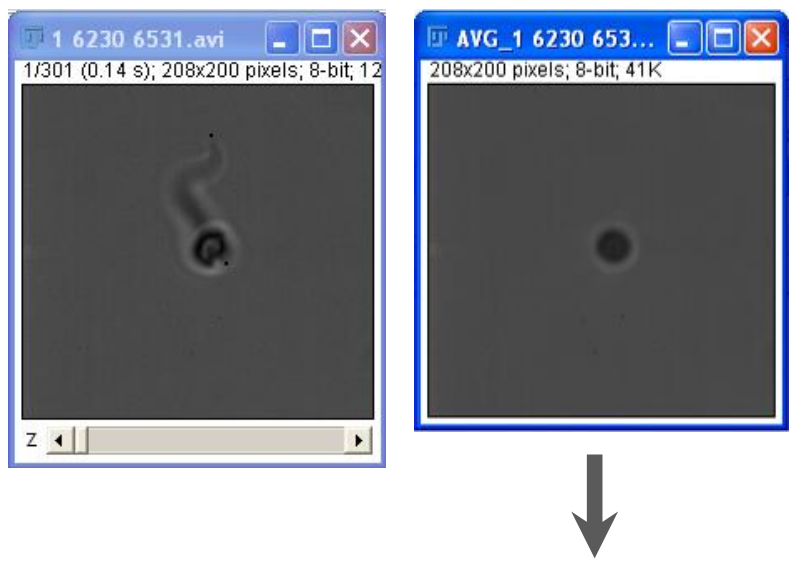

2. Subtract artificial background image form original

- Process

- Image Calculator

- Operation: Difference

3. Detect edges by variance operation

- Process

- Filters

- Variance

- Radius: 5 pixel
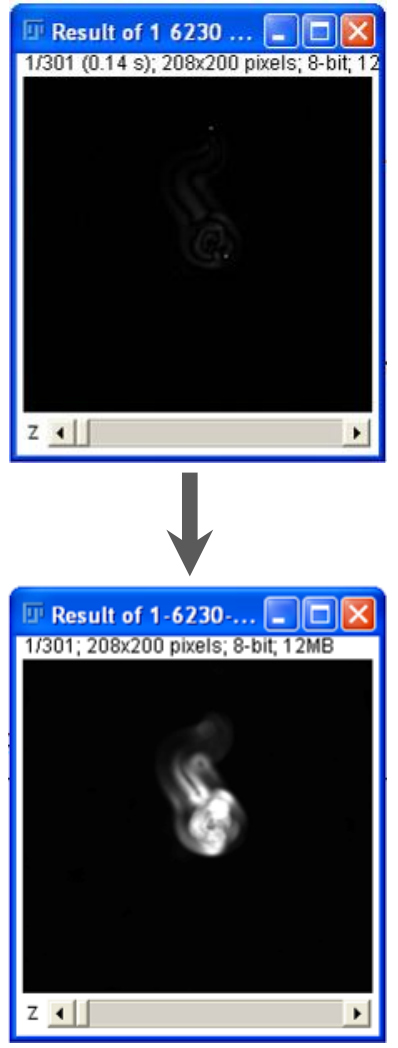

44 | P a g e 
4. Define greyscale threshold with least noise and most signal

- Image

- Adjust

- Threshold

- Set 5-255 e.g.
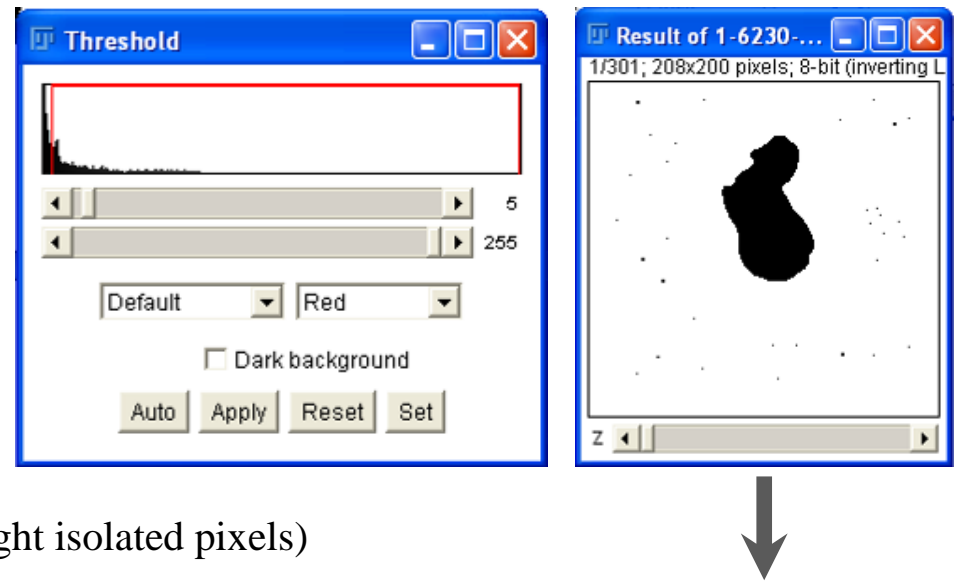

5. Remove noise (dark and bright isolated pixels)

- Process

- Noise

- Despeckle

6. Smooth edges and connected interrupted features

- Process

- Filter

- Gaussian Blur

- radius: 5 pixel
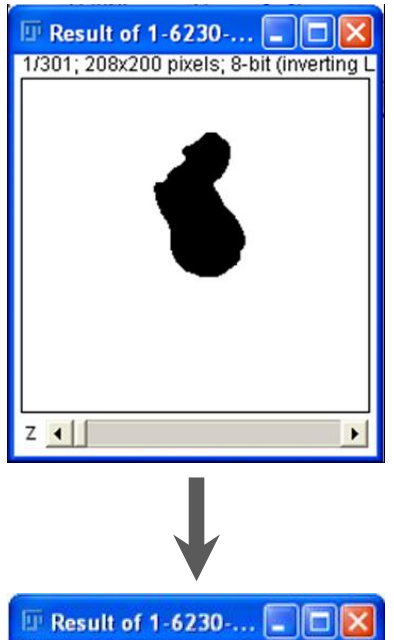

1/301; $208 \times 200$ pixels; 8-bit (inverting L

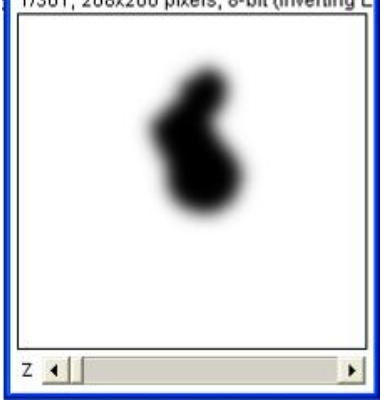

7. Set new threshold

- Image

- Adjust

- Threshold

- Set 175-255 e.g.
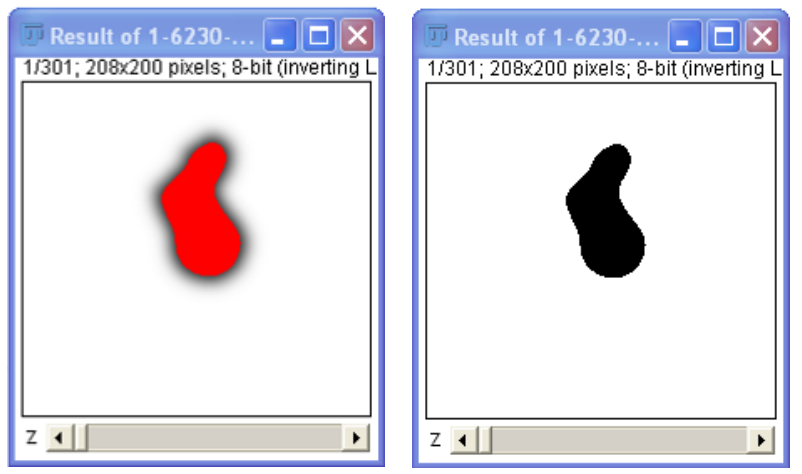
8. Analyze object shape and center of mass postion

- Analysis

- Analyze Particles

- Show: Outline

- Show: ellipse

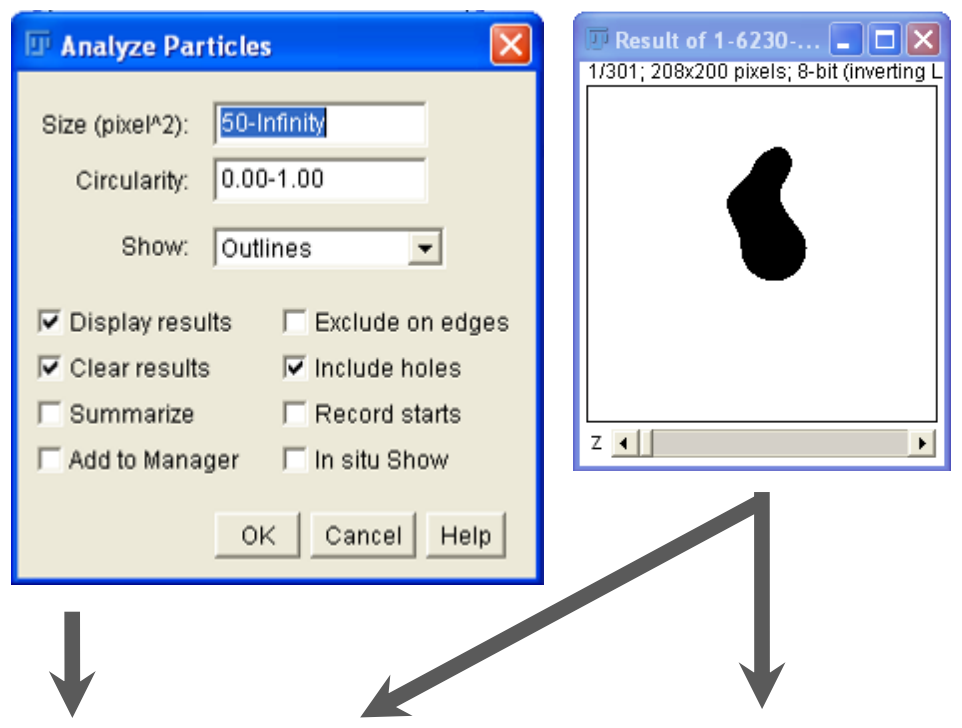

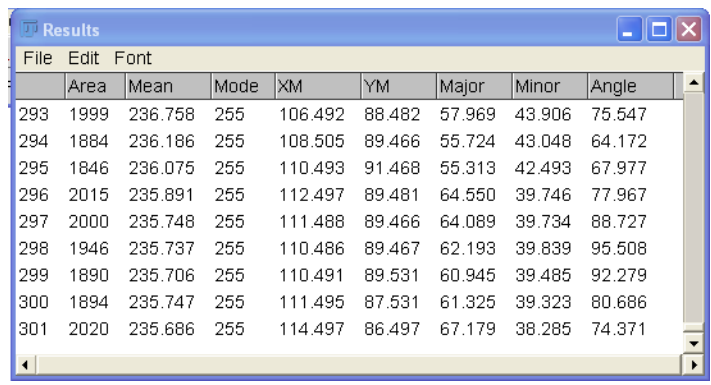

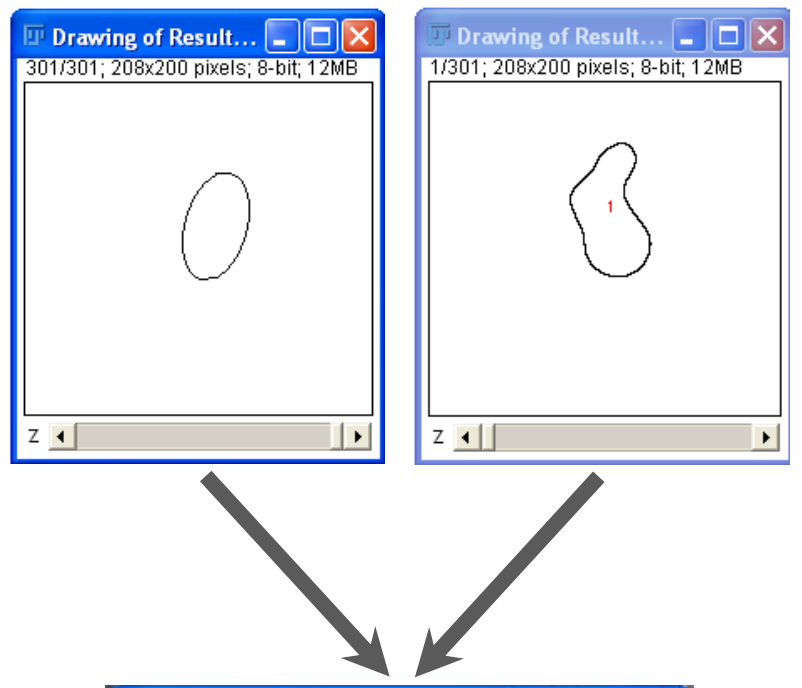

9. Overlay shape descriptors as control

- Image

- Colour

- Merge channels

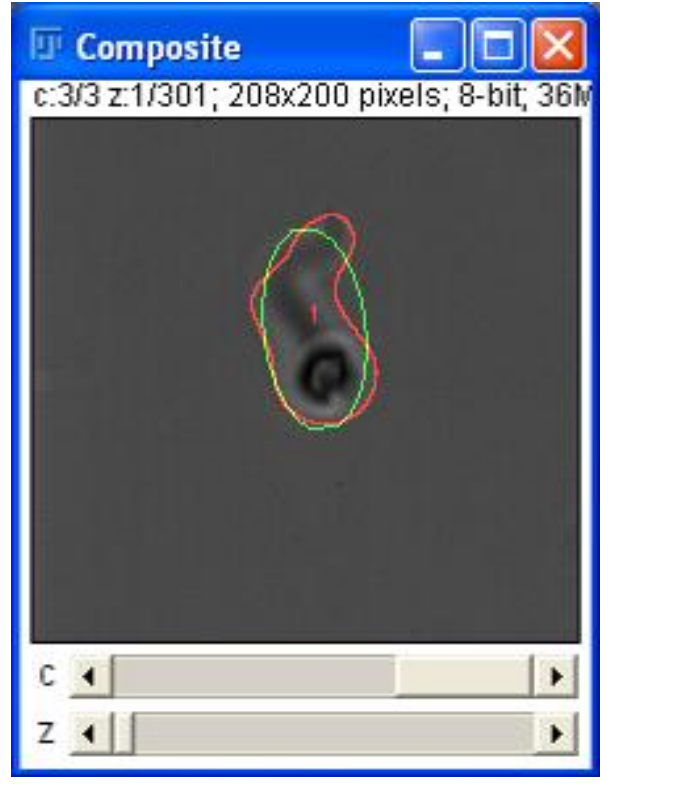

Fig. pages 2-4: Image series of general image processing procedure used for analysis of experimental data. All images were obtained by screen shots whilst running NIH imageJ software. 
It is good scientific practice to optimize the presented method for the experimental focus, but never to change a running procedure for data that is going to be compared. The number of detected objects should always be equal to the expected as a control of analytical accuracy.

As a qualitative control of the obtained data for trypanosome motility, we imaged the hand of a clock and compared both center of mass COM displacements in x-axis direction (Fig. 2.41)

\section{clock hands}

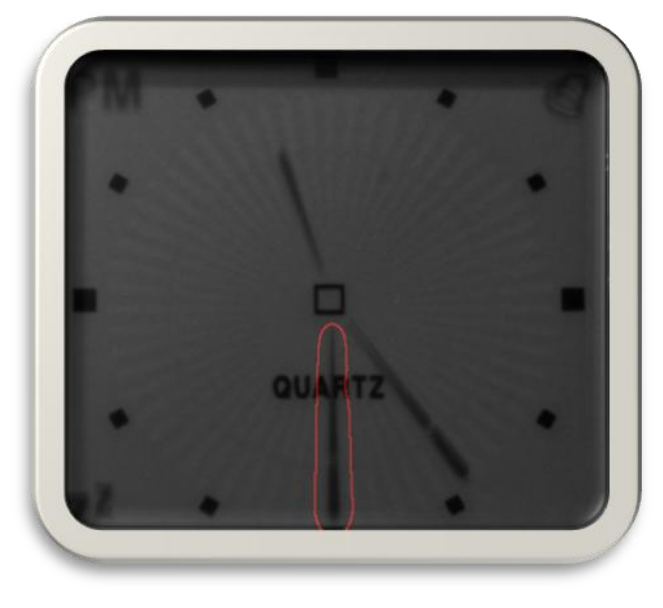

Trypanosome

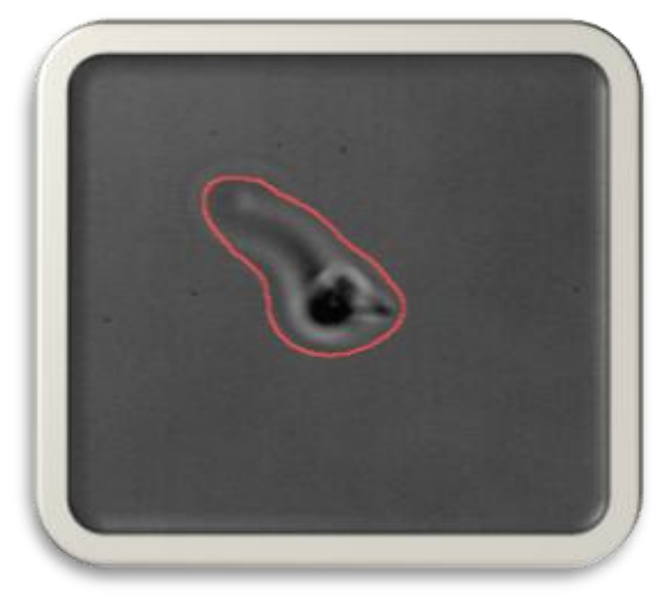

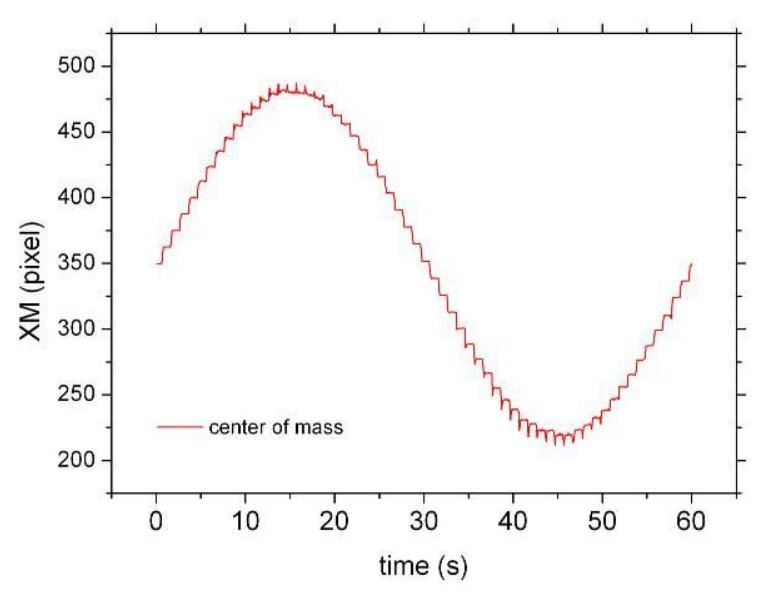

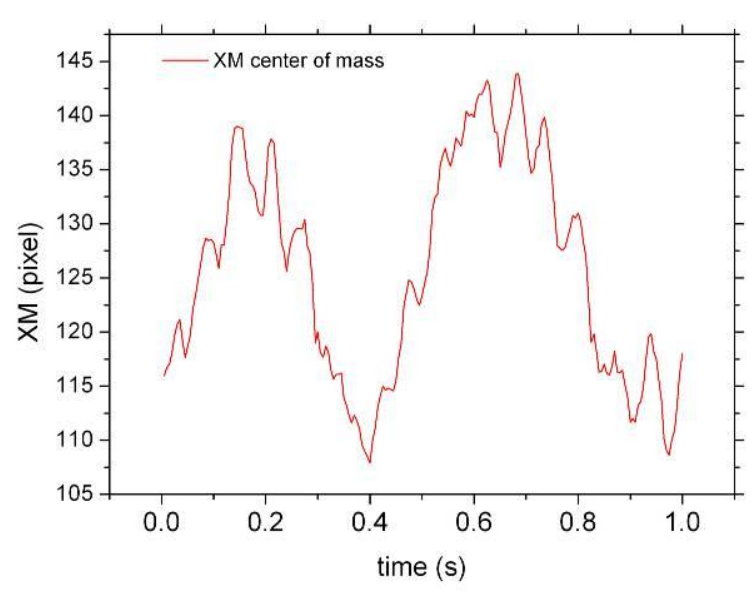

Fig. 2.41: a) Processed images of the second hand of a digital clock and a trypanosome. Red line: shape outline. b) Center of mass traces for the second clock hand and a trypanosome. Red line: com trace.

The expected sinusoidal trace of the clock hand reveals the non continuous motion of the hand, jumping from one second to the other. Similar to the clock hand, the trypanosome COM also undergoes a periodic motion that will be described later on in chapter 4. 


\section{PROCEDURES}

All image processing routines were automated using imageJ macro language. The following pages include a list of all macros written for the experiments of this thesis.

\section{CALIBRATION OF THE OPTICAL TRAP}

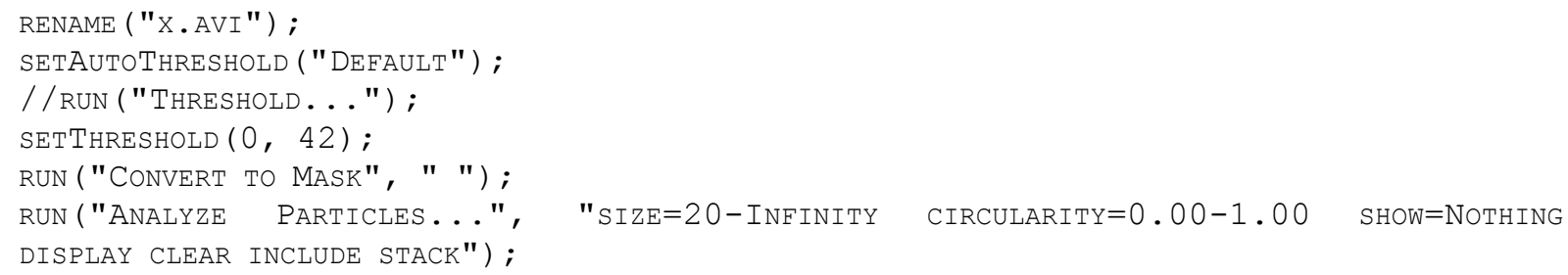

\section{OPTICAL STALL FORCES ON TRYPANOSOMES}

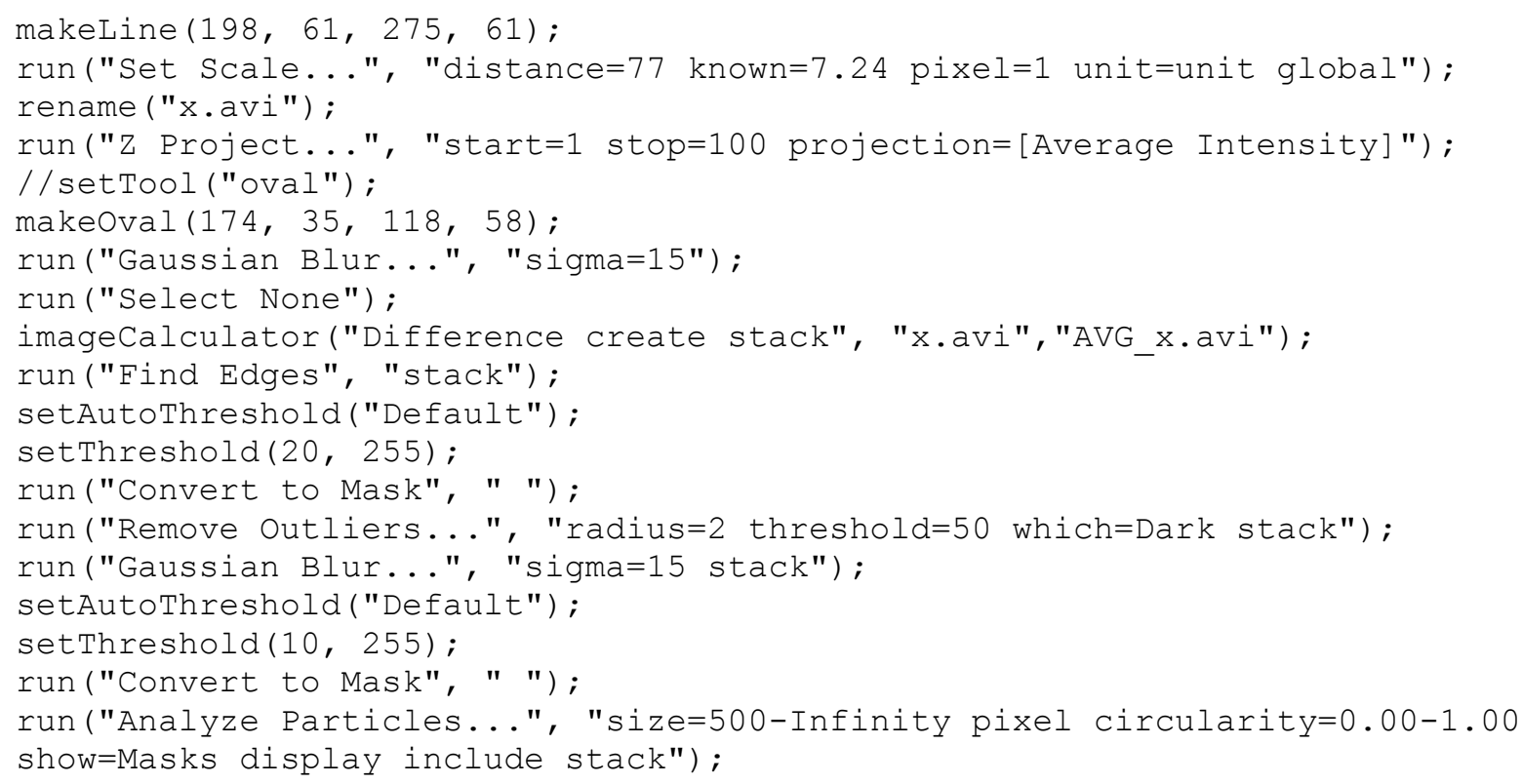

\section{TRAPPING LOCI OF TRYPANOSOMES}

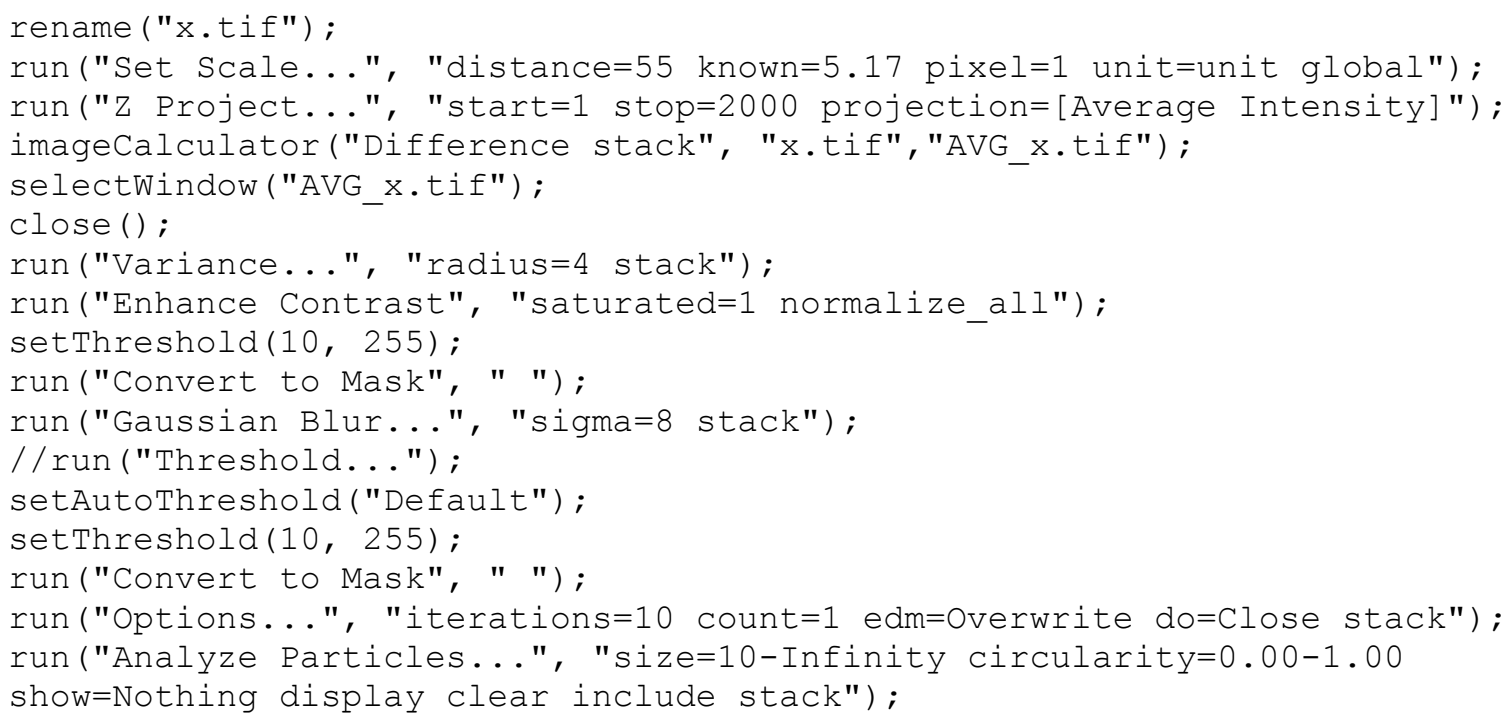


TRYPANOSOME MOTILITY IN OPTICAL CONFINEMENT

See trapping loci of trypanosomes

HYDRODYNAMIC INTERACTIONS OF TRYPANOSOMES

See trapping loci of trypanosomes

PHOTOTOXICITY OF OPTICAL TRAPPING

rename ("x.avi");

run ("8-bit") ;

makeLine $(105,127,203,127)$;

run ("Set Scale...", "distance=98 known=9.2105 pixel=1 unit=unit global");

run ("Select None");

run("z Project...", "start=1 stop=5000 projection=[Average Intensity]");

imageCalculator("Difference stack", "x.avi","AVG_x.avi");

selectWindow("AVG_x.avi");

close ();

run ("Variance...", "radius=4 stack");

run("Enhance Contrast", "saturated=2 normalize_all");

setAutoThreshold ("Default");

/ / run ("Threshold...");

setThreshold $(10,255)$;

run ("Convert to Mask", " ");

run ("Despeckle", "stack");

run("Remove Outliers...", "radius=2 threshold=50 which=Dark stack");

run("Gaussian Blur...", "sigma=8 stack");

setAutoThreshold ("Default");

setThreshold (13, 255);

run ("Convert to Mask", " ");

run("Analyze Particles...", "size=25-Infinity circularity=0.00-1.00 show=Nothing display clear include stack");

TRYPANOSOME MOTILITY IN CONFINEMENT

\section{$2 \mu \mathrm{m}$ and $5 \mu \mathrm{m}$ channels}

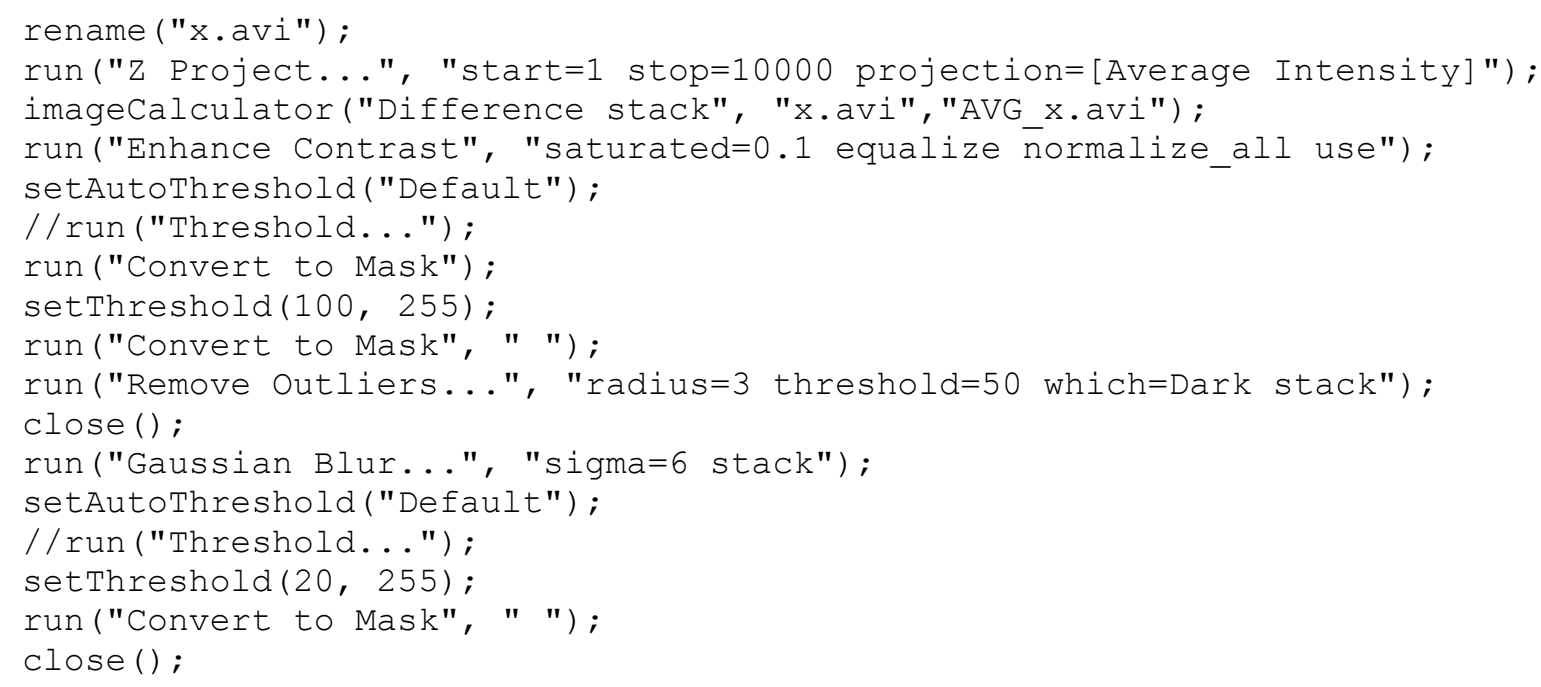

\section{$10 \mu \mathrm{m}$ and $20 \mu \mathrm{m}$ channels}

rename ("x.avi");

run("Grouped ZProjector", "group=10000 projection=[Average Intensity]"); imageCalculator("Difference stack", "x.avi","Projection of AVG x.avi"); setAutoThreshold ("Default");

//run ("Threshold..."); setThreshold (39, 255);

run("Convert to Mask", " ");

run("Remove Outliers...", "radius=1 threshold=50 which=Bright stack");

run("Remove Outliers...", "radius=3 threshold=50 which=Dark stack"); 


\section{TRYPANOSOME CELL CULTURE}

Basic cell biology methods have been applied and combined with specialized trypanosome cell culture methods, provided by M. Engstler's lab (Biocenter, University of Würzburg, Germany). Several molecular biological labeling methods have been adapted for trypanosome labeling, modifying the protocols provided by the manufacturer.

\section{TRYPANOSOMA STRAIN}

Trypanosoma brucei brucei MITat1.2 monomorphic BSF (Cross, 1975)

\section{CULTURE OF MONOMORPHIC BSF TRYPANOSOMES}

The cells were cultured in HMI-9 medium at $37^{\circ} \mathrm{C}, 5 \% \mathrm{CO}_{2}$ in water saturated atmosphere. The cell number was regularly controlled using a Neubauer cell counting chamber and was always kept below $7 * 10^{-5}$ cells per milliliter.

\section{THAWING AND FREEZING OF BSF TRYPANOSOMES}

Thawing: frozen cells were thawed at $37^{\circ} \mathrm{C}$ and resuspended in $9 \mathrm{ml} \mathrm{HMI}-9$ medium. The cells were centrifuged for $10 \mathrm{~min}$ at $1400 \mathrm{~g}$, the supernatant was discarded and the cell pellet was resuspended in fresh HMI-9 medium for cultivation.

Freezing: cells were grown to a density of $7 * 10^{-5}$ cells per milliliter and centrifuged 10 min at $1400 \mathrm{~g}$. The supernatant was discarded and the pellet was resuspended in $1 \mathrm{~mL}$ Glycerol / HMI-9, $1 / 10 \mathrm{v} / \mathrm{v}$ into a cryotube. The cells were slowly cooled down to $-80^{\circ} \mathrm{C}$ within the Strata Cooler and stored in liquid nitrogen

\section{TRYPANOSOME IMMOBILIZATION}

\section{THE PROTOCOL IS GIVEN BY M. ENGSTLER}

Centrifuge $1 \mathrm{~mL}$ fully grown cells $90 \mathrm{~s}$ at $6000 \mathrm{rpm}$. Discard supernatant and resuspend in $1 \mathrm{ml}$ TDB / 2-deoxyglucose buffer, repeat 2 x. Resuspend in TDB / 2-deoxyglucose to a density of $7 * 10^{5}$ cells per $\mathrm{ml}$ and use for experiment

\section{PREPARATION OF MICROFLUIDIC CHANNELS FOR TRYPANOSOME CELLS}

Dilute 5\% BSA in $100 \mathrm{ml}$ TDB buffer w/v and sterile filtrate the solution using a $0.22 \mu \mathrm{m}$ pore size syringe filter. Completely fill the microfluidic channel with the TDB/BSA medium and incubate for $10 \mathrm{~min}$. 


\section{TRYPANOSOME LABELLING \\ ETHIDUM BROMIDE STAINING OF TRYPANOSOMES}

1. Centrifuge $1 \mathrm{ml}$ fully grown cells $90 \mathrm{~s}$ at $6000 \mathrm{rpm}$.

2. Discard supernatant and wash in $1 \mathrm{ml}$ TDB buffer, repeat $2 \mathrm{x}$.

3. Add $1 \mu 10.5 \% \mathrm{EtBr} / \mathrm{TDB}$ solution and incubate on ice for $5 \mathrm{~min}$.

4. Wash twice in TDB and dilute to desired concentration.

5. Analyze fluorescence

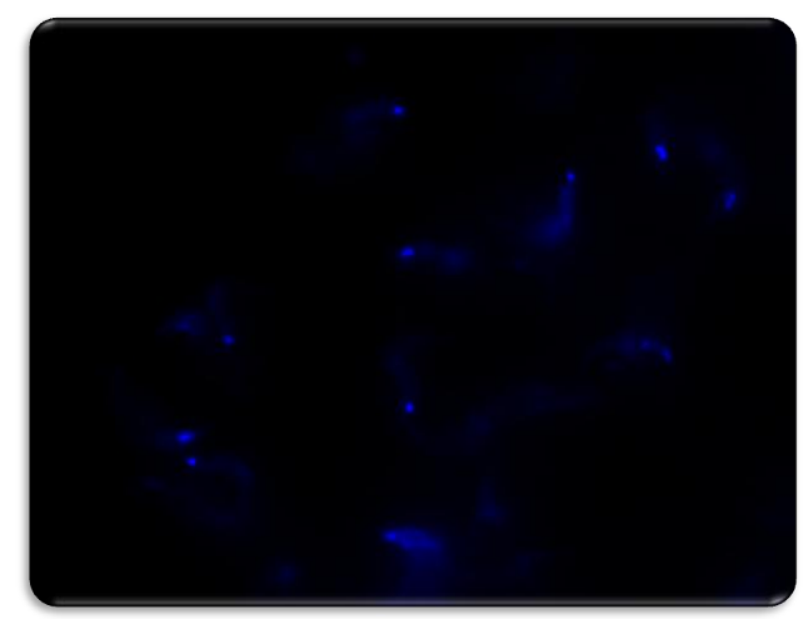

\section{Ethidium Bromide}

Fig. 2.42: Ethidium bromide stain. Image was taken with a PCO sensicam mounted on an Olympus BX 61 microscope using an Olympus PlanApo 100x/1.4 oil immersion objective. Filter set: Ex: 535/40; Em: 574lp. The grayscale image was recolored in blue.

\section{ITK QDOT LABELLING}

THE PROTOCOL IS ADAPTED USING PIERCE PERBIO PROTOCOL

1. Immobilize trypanosomes in $1 \mathrm{~mL} \mathrm{TDB} / 2$-deoxyglucose.

2. Add $2 \mu 1605$ ITK COOH Qdots.

3. Add $4 \mathrm{mg}$ EDC and incubate $15 \mathrm{~min}$ at room temperature RT.

4. Wash Qdots solution in TDB using Sartorius Vivaspin columns.

5. Add $10 \mu \mathrm{l}$ of washed Qdot solution to trypanosome solution and incubate $15 \mathrm{~min}$ RT.

6. Wash 3 times in TDB and analyze fluorescence signal.

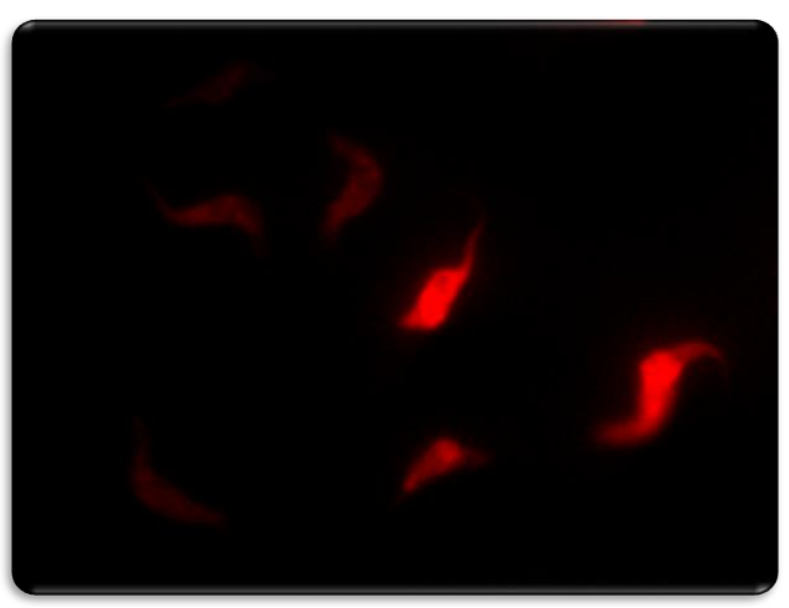

\section{ITK Qdots 605}

Fig. 2.43: ITK Qdot 605 label. Image was taken with a PCO sensicam mounted on an Olympus BX 61 microscope using an Olympus PlanApo 100x/1.4 oil immersion objective. Filter set: Ex: 447/60; Em: 593/44lp. The grayscale image was recolored in red. 


\section{STREPTAVIDIN QDOT LABELLING}

\section{THE PROTOCOL IS ADAPTED USING INVITROGEN PROTOCOL}

1. Immobilize trypanosomes in $100 \mu \mathrm{L}$ TDB / 2-deoxyglucose.

2. Add $10 \mu \mathrm{L}$ Sulfo-NHS-LC-LC-Biotin (1mg / 10mL TDB).

3. Incubate $10 \mathrm{~min}$ at RT.

4. Wash $3 \mathrm{x}$ in TDB and resuspend in $100 \mu 1 \mathrm{TDB} / 2$-deoxyglucose.

5. Add $0.5 \mu \mathrm{L} 585$ Streptavidin Qdots incubate $10 \mathrm{~min}$.

6. Wash $3 \mathrm{x}$ in TDB / 2-deoxyglucose.

7. Resuspend in $100 \mu \mathrm{L}$ TDB / 2-deoxyglucose.

8. Analyze fluorescence.

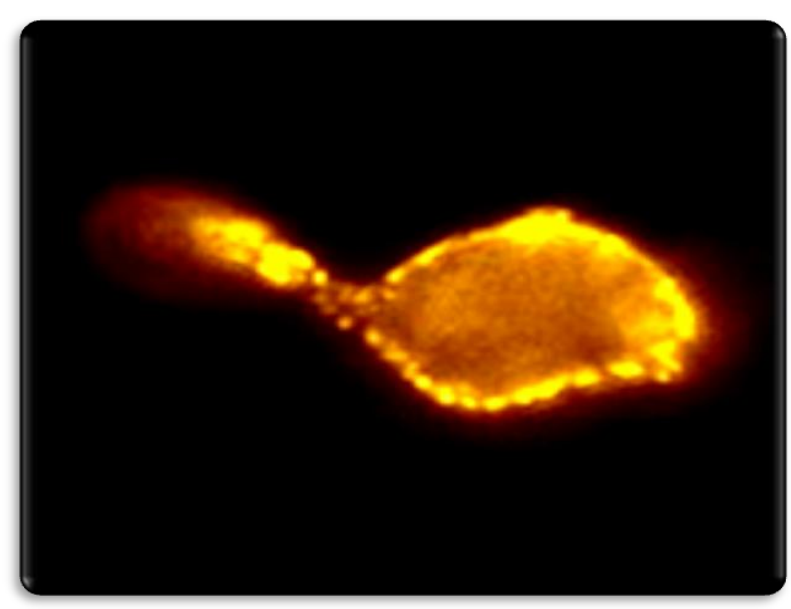

\section{Streptavidin Qdots 585}

Fig. 2.44: Streptavidin Qdot 585 label. Image was taken with a PCO sensicam mounted on an Olympus BX 61 microscope using an Olympus UPlanApo 60x/1.25 oil immersion objective. Filter set: Ex: 535/40; Em: 5721p. The grayscale image was recolored in yellow.

\section{IN SITU NHS-RHODAMIN LABELLING}

\section{THE PROTOCOL IS ADAPTED USING PIERCE PERBIO PROTOCOL}

1. Prepare a microfluidic device coated with BSA.

2. Add $1 \% \mathrm{v} / \mathrm{v}$ of $1 \mathrm{mM}$ NHS-Rohdamin into $1 \mathrm{~mL}$ TDB.

3. Centrifuge $1 \mathrm{~mL}$ fully grown cell $90 \mathrm{~s}$ at $6000 \mathrm{rpm}$.

4. Discard supernatant and resuspend in $1 \mathrm{~mL}$ TDB buffer, repeat $2 \mathrm{x}$.

5. Resuspend in TDB to a density of $7 * 10^{5}$ cells per mL.

6. Inject both Trypanosome TDB and NHS-Rhodamin TDB solutions into the micro channel.

7. Establish a steady fluid focus of both solutions with a fluid velocity $v \leq 40 \mu \mathrm{m} \cdot \mathrm{s}^{-1}$.

8. Trap a trypanosome at full laser power and move it out of the TDB stream into the TDB / NHS-Rhodamin stream, incubate $30 \mathrm{~s}$.

9. Remove trypanosome into the TDB stream and analyze fluorescence signal. 


\section{In Situ labeling}

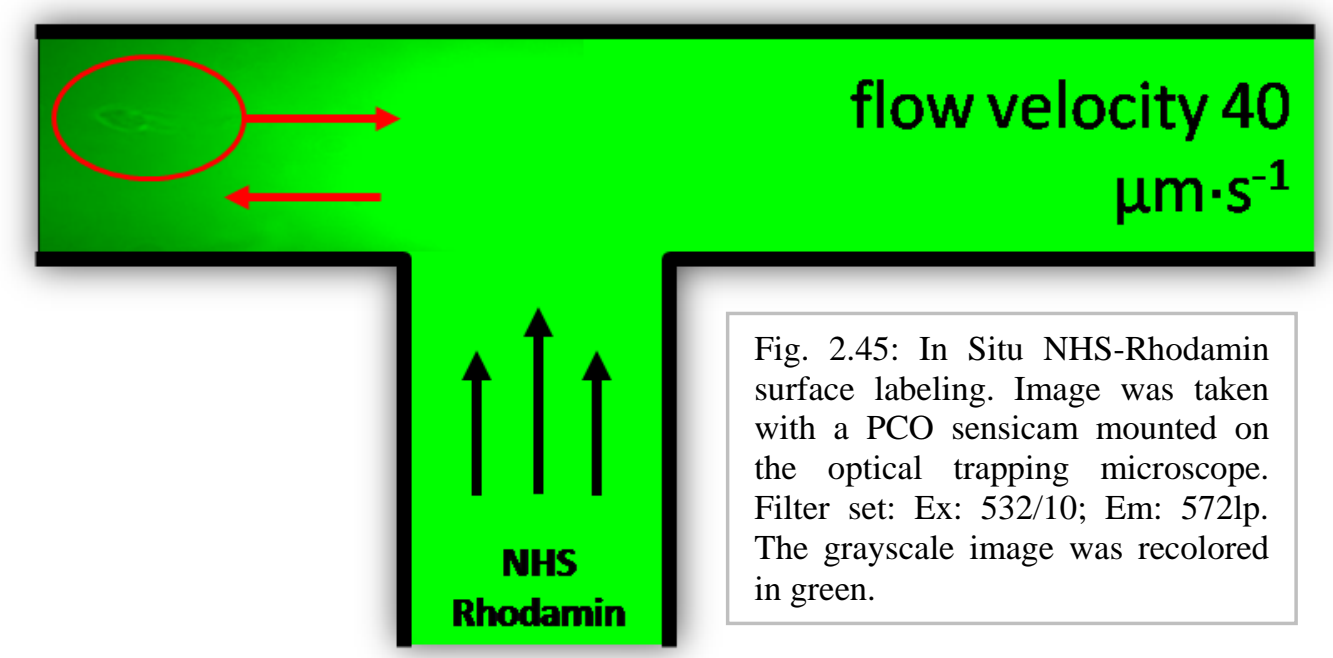

\section{BASIC TRYPANOSOME CULTURE MEDIA}

\section{1fach HMI-9 base stock}

Bidestilled $\mathrm{d} \mathrm{H}_{2} \mathrm{O}$

1. IMDM powder

2. $\mathrm{NaHCO}_{3}$

\section{HMI-9 completing}

HMI-9 base stock

\section{Bathocuproin}

2. Thymidin

3. B-Mercaptoethanol

4. Pen/Strep

5. Hypoxantin

6. FCS

7. Cystein 182

28.2

39.0

Sterilize by filter

136.0

10.0
Add last and recently prepare:

1000

17.66

3.04

$\mathrm{ml}$

$$
\mathrm{g}
$$

9

\section{0}

$\mathrm{ml}$

$\mathrm{mg} / 10 \mathrm{ml}_{2} \mathrm{O}$

10

$\mathrm{ml}$

$\mathrm{mg} / 10 \mathrm{mlH}_{2} \mathrm{O}$

$\mu \mathrm{l} / 10 \mathrm{ml} \mathrm{H}_{2} \mathrm{O}$

10

$\mathrm{ml}$

$\mathrm{mg} / 10 \mathrm{mlH}_{2} \mathrm{O}$

$\mathrm{mg} / 2 \mathrm{OmlH}_{2} \mathrm{O}$

10

10

20

100

$\mathrm{ml}$

$\mathrm{ml}$

$\mathrm{ml}$

$\mathrm{V} / \mathrm{V} \div$

$\mathrm{ml}$

$\mathrm{mg} / 10 \mathrm{mlH}_{2} \mathrm{O}$

10

$\mathrm{ml}$

$\sum$

1000

ml

\section{Freezing medium}

HMI-9 bzw. SDM79

FCS

Glycerin

$(\mathrm{v} / \mathrm{V})$
$(\mathrm{V} / \mathrm{V})$
$(\mathrm{V} / \mathrm{V})$

$\Sigma$
40

50

10

10

$\mathrm{ml}$ 
Trypanosoma dilution buffer TDB

bd $\mathrm{H}_{2} \mathrm{O}$

$\mathrm{pH} 7,7$

$\sum$

1000

ml

$\mathrm{KCl}$

$\mathrm{NaCl}$

$\mathrm{MgSO}_{4}$

$\mathrm{Na}_{2} \mathrm{HPO}_{4} * 2 \mathrm{H}_{2} \mathrm{O}$

$\mathrm{NaH}_{2} \mathrm{PO}_{4} * 1 \mathrm{H}_{2} \mathrm{O}$

Glucose

Sterilize by filter

TDB 2-deoxyglucose buffer

Glucose is replaced by 2-deoxyglucose.

$\begin{array}{ll}0,37 & 9 \\ 4,68 & 9 \\ 0,62 & 9 \\ 3,56 & 9 \\ 0,28 & 9 \\ 3,96 & 9\end{array}$

$0,37 \quad 9$

$4,68-9$

$0,62-9$

$0,28 \quad 9$

g




\section{CHAPTER SUMMARY}

The chapter is comprised of four main parts. Trapping forces determined for trypanosomes are given in the first part, whereas the according trapping positions are described in the second part. The third part includes a description of forces that are generated by trypanosomes, measured by optical trapping methods, whereas the last part gives a measure of phototoxic effects.

\section{OPTICAL TRAPPING OF BIOLOGICAL OBJECTS}

1987 marks the beginning of optical micromanipulation of biomatter and living cells (32). It was when Arthur Ashkin and Joe Dziedzic successfully trapped rod shaped tobacco mosaic plant viruses, when they first realized that photodamage by means of shrinking of the particle is actually less prominent for these than for polystyrene or even silica beads (32). Since photodamage on biomatter is less fatal than expected, it was only natural to also investigate optical trapping of bacteria and even eukaryotic cells.

Optical properties of biomatter are difficult to predict, for the shape of cells is often very irregular and the composition of cellular compartments is not constant at all times. Likewise, orientations and fluctuations of such particles in well defined optical potentials give information about the material itself. Optically anisotropic materials with varying polarizability for example, will have a preferred orientation relative to the polarization of the optical trap.

\section{Optical trapping of a virus}

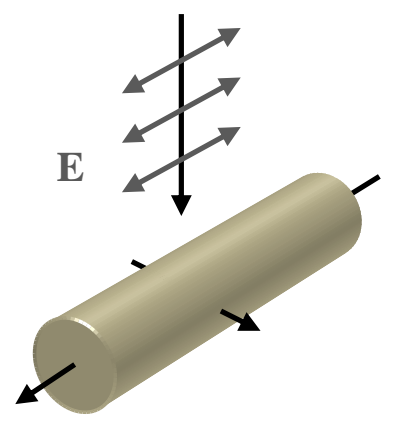

Fig. 3.1: Rod shaped TMV virus within an optical trap. The long axis of the virus aligns parallel to the electric field vector $E$. 
Figure 3.1 illustrates such a rod like virus, where the polarizability of the long axis direction differs strongly from the short axis and so the particle aligns parallel to the electric field vector of the laser beam. With a birefringent particle, not only trapped, but also rotationally aligned, the backward Rayleigh scattering pattern can be used to calculate not only the diameter of a particle, but also the length $\underline{(32)}$.

The given example illustrates the possibilities of optical trapping methods, not only as micromanipulators, but also as analytic tools. In the following experiments, we introduce optical trapping of trypanosomes in the attempt to explore these possibilities. 


\section{OPTICAL TRAPPING OF TRYPANOSOMES}

\section{INTRODUCTION}

Being protozoans trypanosomes share many features with other eukaryotic cells, such as a nucleus, various cell organelles embedded in a complex cytoskeleton and an outer membrane, covered with glycoprotein (33). In an overall way their cytosol should be much like that of any other eukaryotic cell which has been successfully manipulated by optical tweezers ( $\underline{34}$, $\underline{35}, \underline{28})$. However, trypanosomes are single cell organisms that are highly specialized to live and proliferate in a bloodstream environment, therefore their cellular structure is unique in many ways. Unlike other flagellates, the trypanosome is rapidly changing cell shape to mediate propagation forces of a flagellum which is not free standing, but completely attached to the cell. Furthermore, the cell body itself is supported by a microtubule skeleton, different to the actin cortex that supports the outer membrane of any other recently trapped eukaryotic cell $\underline{(36)}$.

Here it is shown for the first time that optical micromanipulation and trapping of trypanosomes is possible at reasonable low laser power and with only marginal phototoxic effects (Fig 3.2). Several methods have been developed to manipulate and control living trypanosomes within microfluidic experiments, examine their motility and measure the forces they generate.

\section{Optically trapped trypanosome}

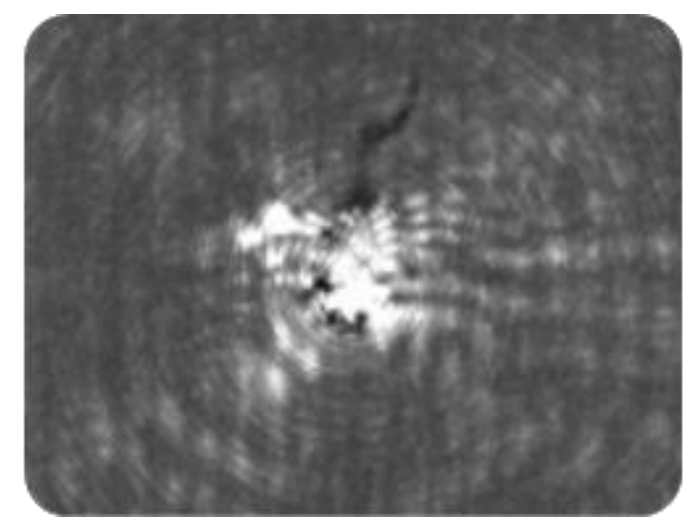

Fig. 3.2: The image shows the laser scattering pattern of an optically trapped trypanosome in brightfield microscopy where the IR filter has been removed.

Consequently, the first part of this chapter concentrates on the description of optical forces that act on trypanosomes, while the second part describes the forces trypanosomes generate themselves. The last part includes a description of phototoxic effects on trypanosome motility as a measure of vitality. During description and analysis of the results, the emerging possibilities of optical trapping methods on trypanosome research are highlighted. 


\section{OPTICAL STALL FORCES ON TRYPANOSOMES}

\section{INTRODUCTION}

A common method to determine optical forces on micron sized particles is to produce a well defined counterforce by fluid flow and to measure the effect on the particle, as described in chapter 2.24. In order to calibrate optical forces that act on trypanosomes via the Stokes drag method, one needs to know the friction coefficient of the cell which is, unlike a sphere, complex in shape. For spherical particles in low Reynolds number environment, the Stokes drag friction coefficient $\beta$ is given by

$$
\beta=6 \pi \eta r
$$

with $\eta$ the dynamic viscosity of the medium and $r$ the radius of the particle. For rod shaped objects with the length $L \gg r$, the drag coefficient can be approximated by

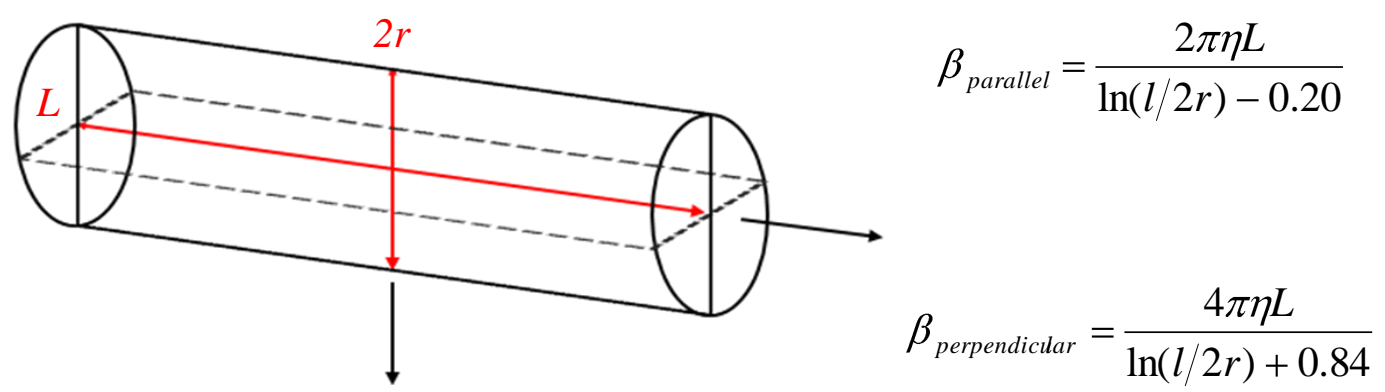

dependent on the orientation of the rod to the flow direction ( $\underline{37})$. In a first approximation the outstretched, slender type, BSF trypanosome can be described as such a rod shaped object which has a length of $L=20 \mu \mathrm{m}$ in length and a radius of $r=1.5 \mu \mathrm{m}$ (as above). The friction coefficient of a trypanosome cylinder, moving in long axis direction, is $\beta=7.4 * 10^{-8} \mathrm{Kg} \cdot \mathrm{s}^{-1}$, corresponding to a sphere with a radius of $r=3.9 * 10^{-6} \mathrm{~m}$. A living, mobile trypanosome however, as it will be shown later in this chapter, is rather bent than stretched out, more similar to a spheroid than to a rod. The friction coefficient of a spheroid can be calculated using the Perrin factor $(1934, \underline{38})$ equation, given by

$$
S=2 \frac{\tanh ^{-1} \xi}{\xi} \quad \text { where } \quad \xi=\frac{\sqrt{\left|p^{2}-1\right|}}{p}
$$


where $\xi$ can be calculated with $p$, the ratio of the long axis $z$ over the short axis $x$ of a prolate spheroid. The two short axis $x$ and $y$ are of the same length but shorter than the axis of revolution.

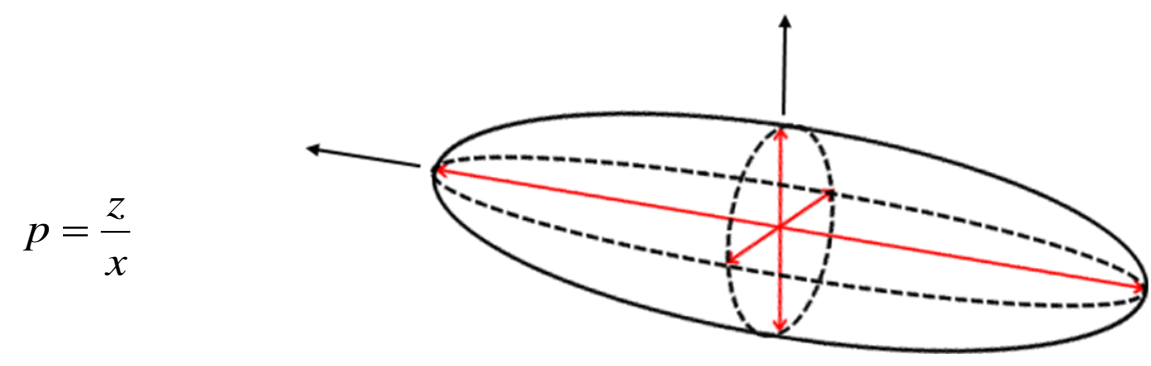

The Perrin translational friction factor $\beta_{\text {Perrin }}$ is then given by

$$
\beta_{\text {Perrin }}=\frac{2 p^{2 / 3}}{S}
$$

With $\beta_{\text {Perrin }}$ known, the effective friction coefficient of such a spheroid, moving in $z$-axis direction, can be calculated by multiplication of the Perrin translational factor $\beta_{\text {Perrin }}$ with the friction coefficient of an arbitrary sphere of a volume equal to the spheroid.

$$
\beta_{\text {spheroid }}=\beta_{\text {sphere }} \beta_{\text {Perrin }}
$$

A trypanosome spheroid with the dimensions $x=1.5 * 10^{-6} \mathrm{~m}$ and $z=10 * 10^{-6} \mathrm{~m}$ has a volume of $V=9.42 * 10^{-17} \mathrm{~m}^{3}$ equal to a sphere with a radius of $\mathrm{r}=2.8 \mu \mathrm{m}$ and a friction coefficient of $\beta_{\text {sphere }}=5.32 * 10^{-8} \mathrm{Kg} \cdot \mathrm{s}^{-1}$. Multiplied with a Perrin translational factor of 1.36 , the effective friction coefficient of a trypanosome is $\beta_{\text {tryp }}=7.21 * 10^{-8} \mathrm{Kg} \cdot \mathrm{s}^{-1}$, equal to a sphere of $r=3.83$ $* 10^{-6} \mathrm{~m}$ in radius.

The development of drag coefficients calculated for spheres, spheroids and rods over increasing length (diameter in case of the sphere) is plotted in (Fig. 3.3). The friction coefficient of a prolate spheroid (red line) develops similar to a rod, moving parallel to the flow (blue line) at high aspect ratios $(z>>x)$ and intercepts at zero. At values $(z>x)$ the rod friction condition is not valid any more, also seen by the steep increase of both black and blue curves. 


\section{Drag coefficients}

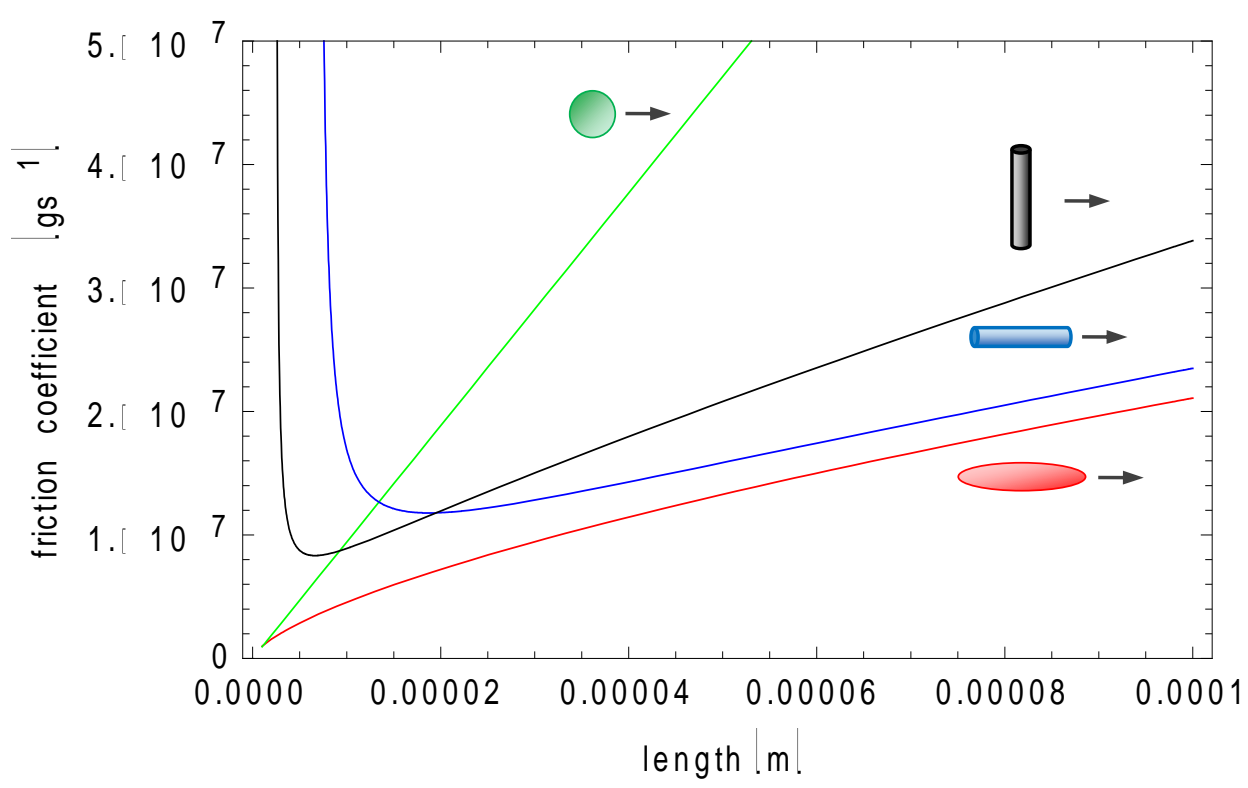

Fig. 3.3: Development of drag coefficient in dependency to cell length (diameter kept constant at $3 \mu \mathrm{m}$ ). Green line: sphere; black line: rod moving perpendicular to the flow; blue line: rod moving parallel to the flow; red line: prolate spheroid. The according particle shapes are indicated by sketches matching the color

However, as a part of the results, it will be seen that the shape of a living trypanosome cannot be described by $r \ll L$, thus a rod like approximation is not valid. Consequently, the Perrin friction factor for prolate spheroids, where $r<L$ is used to calculate the in vivo trypanosome friction factor and to derive the optical stall forces, acting on trypanosomes.

\section{EXPERIMENTS}

All optical stall force experiments on trypanosomes have been conducted within microfluidic flow channels of a width of $500 \mu \mathrm{m}$ and a height of $65 \mu \mathrm{m}$. The cells have been trapped $25 \mu \mathrm{m}$ below the cover slip surface to assure all degrees of free cell motility throughout the entire experiment. Calibration measurements were done at the same distance, using polystyrene spheres of $r=1.553$. The large width of the channels was chosen to establish well defined flow rates which were controlled by high precision syringe pumps.

\section{PREPARATION OF CELLS}

Trypanosoma brucei brucei were cultivated in $\mathrm{HMI}-9$ medium at $37^{\circ} \mathrm{C}, 5 \% \mathrm{CO}_{2}$ and harvested at a density of $7 * 10^{5}$ cells per millilitre. Cells were washed thrice in $1 \mathrm{~mL}$ TDB and resuspended in $1 \mathrm{~mL}$ TDB. The PDMS microfluidics devices were rinsed with $5 \%$ w/v BSA in TDB to prevent the cells from sticking to the walls. 
The cells were injected into the micro channel with disposable $1 \mathrm{~mL}$ plastic syringes. Flow was established using a $5 \mu \mathrm{L}$ Hamilton syringe, driven by a Nexus nemsys syringe pump. The flow rate was set to a slow linear ramp to prevent from relaxation oscillations of PDMS and dissolved gasses. Starting at 0 going up to $7 \mu \mathrm{L} \cdot \mathrm{h}^{-1}$ in $60 \mathrm{~s}$, the change in flow velocity whilst the event of cell escape $(<0.5 \mathrm{~s})$ is negligible $(<5 \%)$. The time of measurement was restricted to $30 \mathrm{~min}$ ex vitro in order to prevent major metabolic and morphologic changes of the cells.

\section{Preparation of IMmobilized Cells}

Trypanosoma brucei brucei were cultivated in $\mathrm{HMI}-9$ medium at $37^{\circ} \mathrm{C}, 5 \% \mathrm{CO}_{2}$ and harvested at a density of $7 * 10^{5}$ cells per millilitre. Cells were washed thrice in $1 \mathrm{~mL}$ TDB and resuspended in 1ml 2-deoxy-D-glucose TDB and incubated for $15 \mathrm{~min}$. The cell transfer into the microfluidic device takes an average of 10 min preparation time which was considered as extra incubation time. The cells were only analyzed when active motility ceased to appear.

\section{OPTICAL MANIPULATION OF THE CELLS}

The cells were trapped at different laser powers, starting from $4 \mathrm{~mW}$ for immobilized cells and $15 \mathrm{~mW}$ for active cells, going up to the maximum available power of $27 \mathrm{~mW}$ at the sample. The escape velocities were recorded with a Phantom Miro 3 high speed camera at a rate of $20 \mathrm{fps}$. The center of mass was derived using automated ImageJ velocimetry methods as described in chapter 2 . The cell shapes were determined exactly one frame before they got dragged out of the trap. 100 immobilized and 30 active cells were measured in total.

\section{REFRACTIVE INDICES}

Refractive index measurements have been conducted $\left(20^{\circ} \mathrm{C}, 532 \mathrm{~nm}\right)$ using a Zeiss refractometer model 144930. Water: 1.333; TDB: 1.335; TDB: deoxy 1.348 .

\section{DYNAMIC VISCOSITY MEASUREMENTS}

Viscosity measurements of all cell culture media involved in cell trapping have been performed in an Ubeholde viscosimeter Ref. No.: $537 \quad 10 / 1$ and $53720 / 1$ at $20^{\circ} \mathrm{C}$. Water: $0.97 \mathrm{cP} \cdot \mathrm{s}$, TDB: $1.00 \mathrm{cP} \cdot \mathrm{s}$; TDB 2-deoxy-D-glucose: $0.99 \mathrm{cP} \cdot \mathrm{s}$. 


\section{RESULTS AND DISCUSSION}

\section{TRYPANOSOME CELL SHAPE}

Trypanosome motility is characterized by rapid deformations of the cell body. Trypanosomes are often measured by their maximum cellular expansions which are referred to be $D=3 \mu \mathrm{m}$ in diameter and $L=20 \mu \mathrm{m}$ in length (16).

We analyzed living BSF trypanosomes in order to obtain a good measure of their actual expansions and to calculate their friction coefficients.

\section{Trypanosome cell shape}
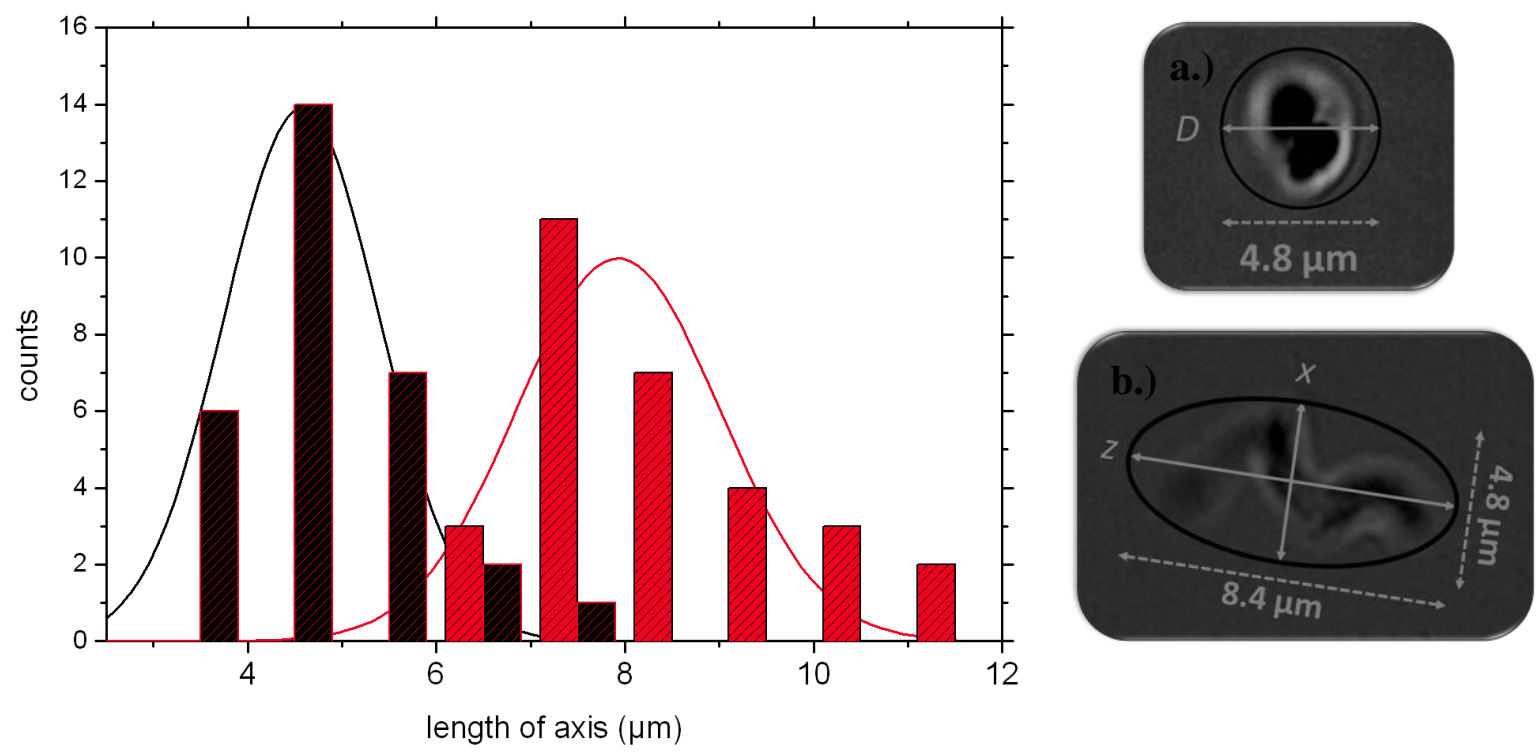

Fig. 3.4: Cell axis length distribution. Black bars: minor axis; red bars: major axis. The black and red lines show Gaussian fits to the histograms with $4.8 \mu \mathrm{m}$ for the minor and $8.4 \mu \mathrm{m}$ for the major axis length. a) Planar view of a cell; b) axial view of a cell obtained without flow. Number of samples $n=30$

The mean cell expansion of living trypanosomes, as seen in our experiments, is $z=8.4 \mu \mathrm{m}$ for the major axis and $x=4.8 \mu \mathrm{m}$ for the minor axis, with a standard deviation of $\sigma_{z}=1.3 \mu \mathrm{m}$ and $\sigma_{x}=0.9 \mu \mathrm{m}$ respectively. We conclude that the mean $B S F$ trypanosome cell expansions in vivo are very different to the outstretched cell dimensions and have to be considered for further calculations. With the radial diameter of a cell $D \cong x$ (Fig. 3.4b), the friction coefficient of a spheroid like trypanosome can be calculated. We get $\beta_{\text {real }}=5.6 * 10^{-8} \mathrm{~kg} \cdot \mathrm{s}^{-1}$ for active swimming cells in water like medium at a temperature of $20^{\circ} \mathrm{C}$ which is significantly smaller, compared to $\beta_{\text {stretched }}=7.21 * 10^{-8} \mathrm{~kg} \cdot \mathrm{s}^{-1}$, calculated for an outstretched cell. 


\section{ORIENTATION IN FLOW}

Living trypanosomes were successfully trapped within a microfluidic flow chamber at different laser powers. A linear flow ramp was repeatedly applied via a syringe pump and the cells were recorded until they got dragged out of the trap. The resulting cell trace was used to determine the actual flow velocity at which the cells got dragged out of the trap.

\section{optical trap}

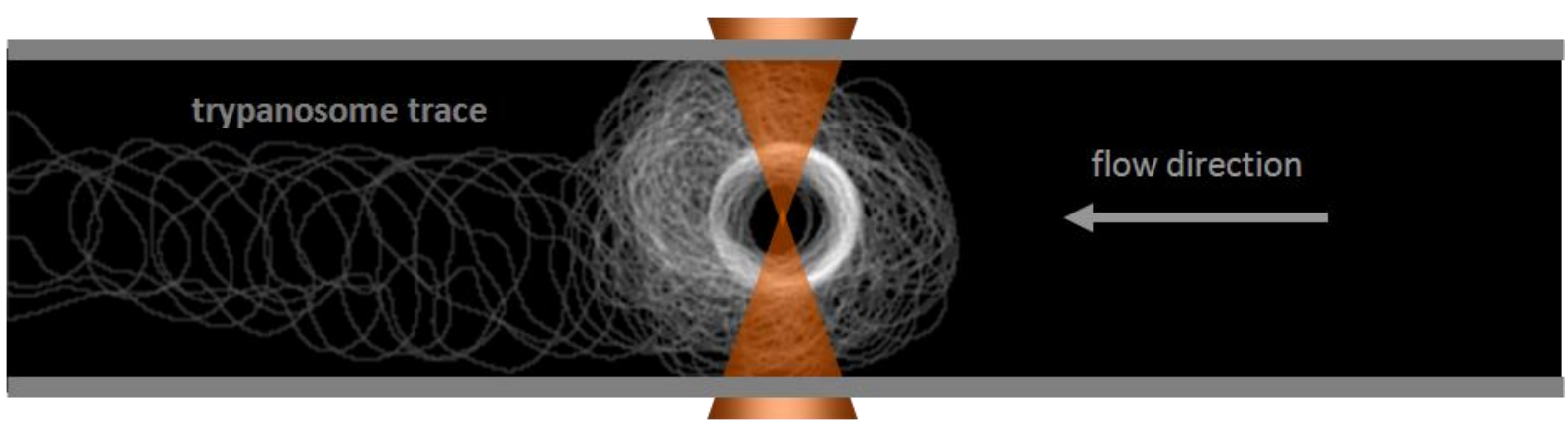

Fig. 3.5: Trace of an optically trapped trypanosome from the onset of fluid flow (gray arrow) until the event of trap escape.

Increasing fluid velocities we found the trypanosomes aligned, owing to the flow, before they are dragged out. Without flow, trypanosomes were able to move freely within the boundaries of the optical confinement (Fig. 3.6a). At a velocity of about $v_{\text {fluid }}>20 \mu \mathrm{m} \cdot \mathrm{s}^{-1}$ every cell got more or less aligned to the flow (Fig. 3.6b).
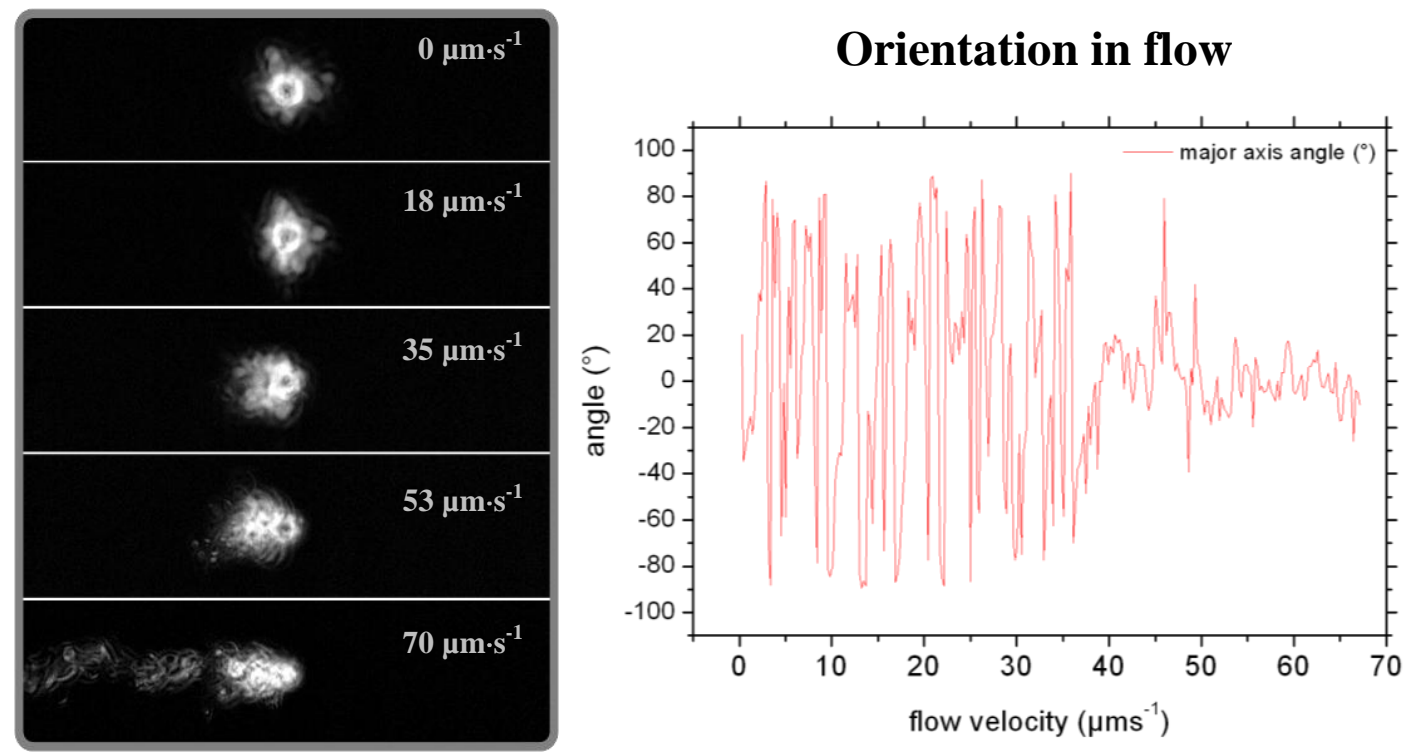

Fig. 3.6: a) Trypanosome orientation in flow (40 frames overlay). b) Orientation of the cell in flow given by major axis angle.

At velocities higher than $v_{\text {fluid }}>40 \mu \mathrm{m} \cdot \mathrm{s}^{-1}$, the cells cannot realign against the flow anymore and they are finally dragged out of the trap. Both cell orientations, anterior or posterior end first, have been observed, but the long axis is always directed parallel to the flow. 
Friction of asymmetric particles, like a rod, or a spheroid, depends on orientation relative to the flow and is highest with the long axis perpendicular towards it. Since the trapped cells always align parallel to the flow, we can assume that the dynamic friction pattern of trypanosomes is asymmetric, as we have determined for the cell shape in the snap shots given in figure 3.6.

\section{OPTICAL STALL FORCE ON TRYPANOSOMES}

With the friction coefficients given for both, immobilized and active cells, the optical stall forces can be calculated and compared (Fig. 3.7). The minimum stall velocity $v=28 \mu \mathrm{m} \cdot \mathrm{s}^{-1}$ at which the cells are dragged off the trap is in good agreement with the average swimming velocity, reported for BSF Trypanosoma brucei brucei in literature (39) and represents the minimum laser power $P=13 \mathrm{~mW}$ needed to continuously trap a trypanosome in this experiment.

\section{Trypanosome escape velocities}

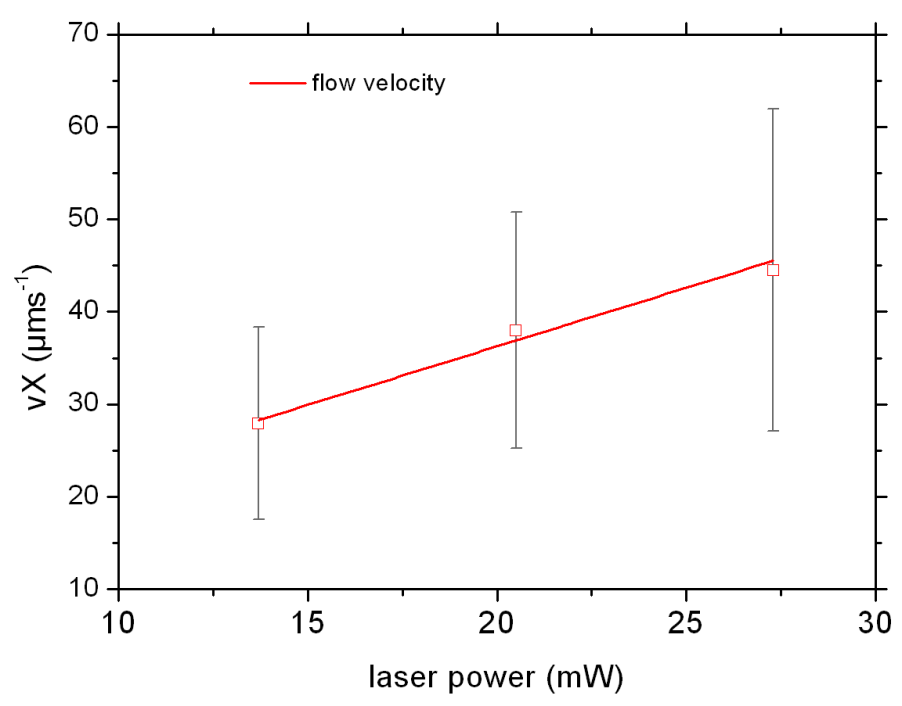

Fig. 3.7: Flow velocities at drag off events for different laser powers at the sample. Number of samples $n=$ 30. Black squares: mean velocities; gray lines: error bars as $\sigma$ of the mean; red line: linear fit to data (slope: $1.27 \mathrm{~mW} \cdot \mu \mathrm{m} \cdot \mathrm{s}^{-1}$, intercept: $10.9 \mu \mathrm{m} \cdot \mathrm{s}^{-1}$ )

Optical stall forces on trypanosomes are strong enough to withstand flow velocities up to $44 \mu \mathrm{m} \cdot \mathrm{s}^{-1}$ at this distance to the cover slip. To maintain freedom of motility, the cells were trapped $25 \mu \mathrm{m}$ below the coverslip. At smaller distances, e.g. $5 \mu \mathrm{m}$ below the coverslip, an increase of the stall force by the factor of 3 is to be expected.

The optical stall force is derived by multiplication of the velocities given in figure 3.7 with the corresponding Perrin friction factor $\left(\beta_{\text {tryp }}=4.98 \cdot 10^{-8} \mathrm{~kg} \cdot \mathrm{s}^{-1}\right)$ for a temperature of $25{ }^{\circ} \mathrm{C}(\eta=$ $0.89 \mathrm{mPa} \cdot \mathrm{s}^{-1}$ ) within the system (Eq. 3.6). 


\section{Optical stall force on trypanosomes}

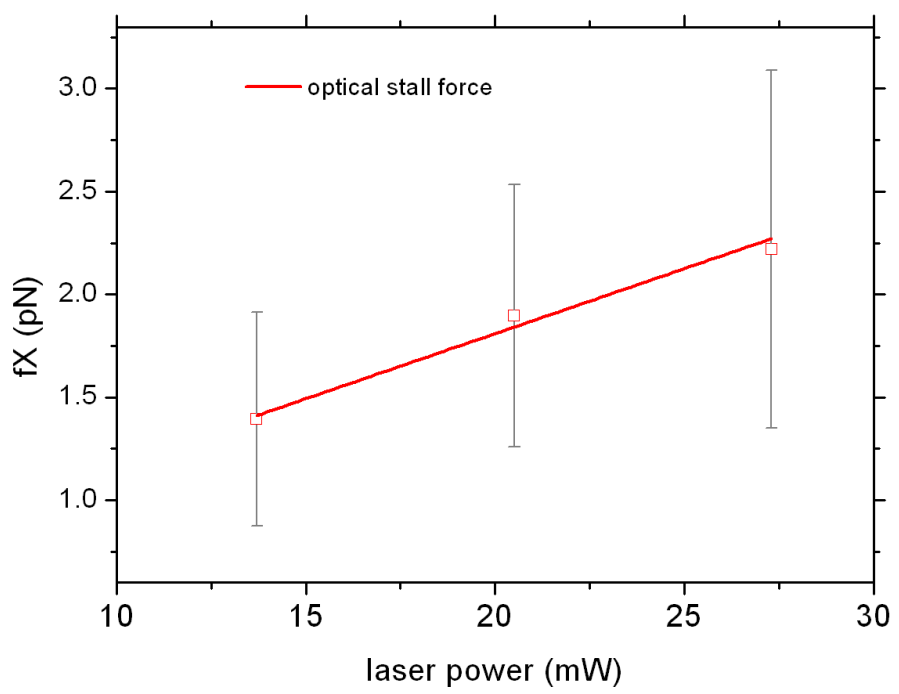

Fig. 3.8: Optical stall force on living trypanosomes in TDB medium at $25^{\circ} \mathrm{C}$. Number of samples $n=30$. Red circles: mean stall forces; gray lines: error bars $(\sigma)$; red line: linear fit (slope: $63 \mathrm{fN} \cdot \mathrm{mW}^{-1}$; interception: 0.55 $\mathrm{pN})$

Optical stall forces on living $B S F$ trypanosomes have been determined with $F_{\text {Stall }}=63 \mathrm{f} \mathrm{N} \cdot \mathrm{mW}^{-1}\left( \pm 7.7 \mathrm{fN} \cdot \mathrm{mW}^{-1}\right)$ using a $808 \mathrm{~nm}$ laser at a distance of $25 \mu \mathrm{m}$ below the coverslip surface. In order to distinguish fluid drag from trypanosome propulsion forces that might have contributed to the stall force experiments, the experiment was repeated with immobilized cells.

Escape velocities of immobilized cells

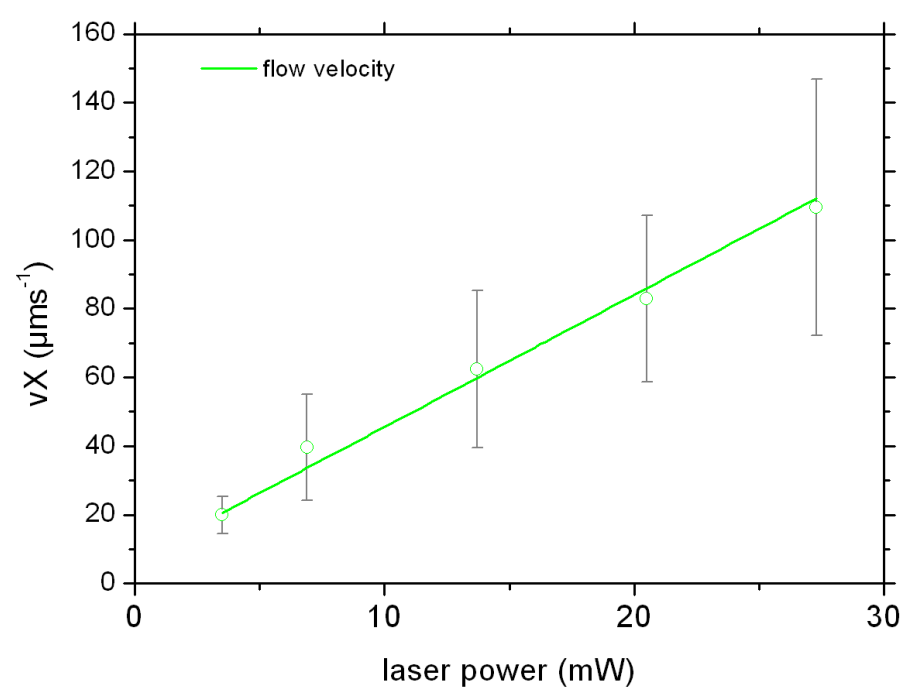

Fig. 3.9: Flow velocities at drag off events for different laser powers at the sample. Number of samples $n=$ 100. Green squares: mean velocities; gray lines: error bars $(\sigma)$; green line: linear fit to data (slope: 3.85 $\mathrm{mW} / \mu \mathrm{m} \cdot \mathrm{s}^{-1}$, interception: $7.1 \mu \mathrm{m} \cdot \mathrm{s}^{-1}$ )

Immobilized cells were successfully trapped with a minimal laser power of $P=3 \mathrm{~mW}$ and maintained trapped at flow velocities up to $100 \mu \mathrm{m} \cdot \mathrm{s}^{-1}$, using a maximum laser power of $P=27 \mathrm{~mW}$. At shorter distances to the coverslip, we expect the cells to maintain trapped at 
flow velocities up to $300 \mu \mathrm{m} \cdot \mathrm{s}^{-1}$, using inexpensive diode lasers with output powers below $200 \mathrm{~mW}$. This highlights the possibilities of optical trapping, not only as a tool for motility studies on living cells, but also as a tool for micromanipulation within advanced microfluidic flow experiments and measurements of forces at the pico-Newton scale, as to be shown later on.

To calculate the optical stall forces of immobilized BSF trypanosomes, the cell morphology was analyzed as described in order to obtain the actual friction coefficient.

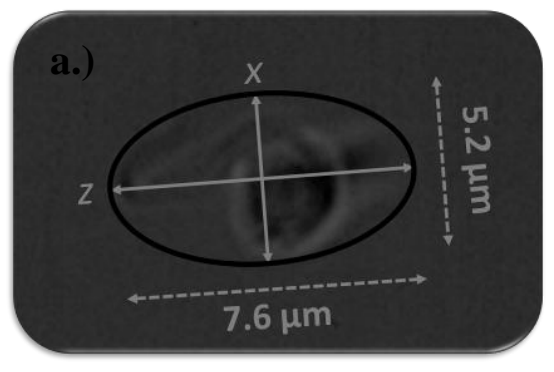

\section{Immobilized cell shape}

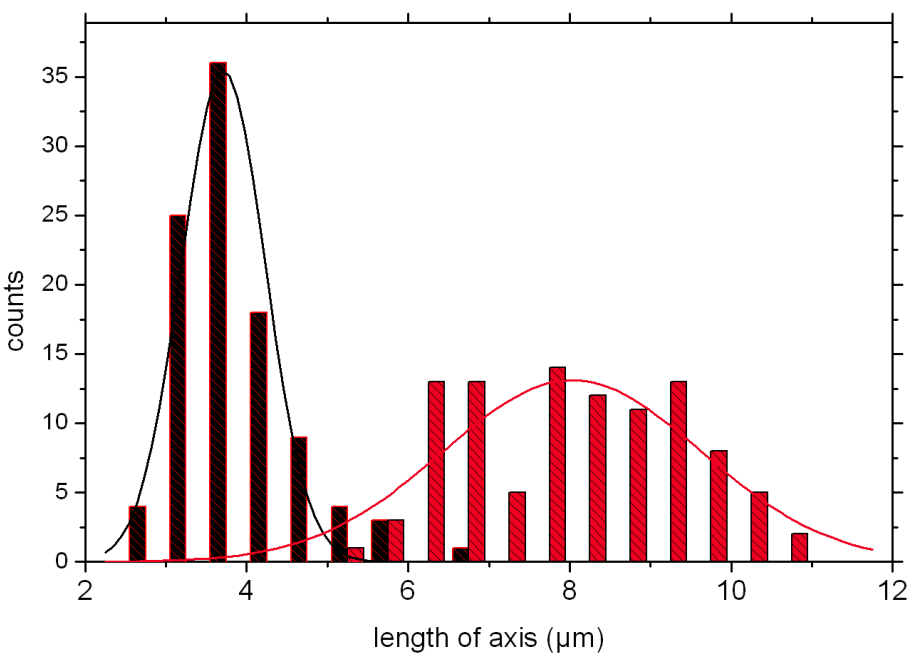

Fig. 3.10: Cell axis length distribution. Black bars: minor axis; red bars: major axis. The black and red lines show Gaussian fits to the histograms with $x=3.9 \mu \mathrm{m}$ for the minor and $z=8.1 \mu \mathrm{m}$ for the major axis length. a): morphology of an immobilized cell. Number of samples $n=100$

The mean cell expansions of immobilized trypanosomes were determined with $z=8.1 \mu \mathrm{m}$ for the major axis and $x=3.9 \mu \mathrm{m}$ for the minor axis, with a standard deviation of $\sigma_{z}=1.1 \mu \mathrm{m}$ and $\sigma_{x}=0.95 \mu \mathrm{m}$. The resulting mean drag coefficient of immobilized trypanosome spheroids at $25^{\circ} \mathrm{C}$ in TDB medium is $\beta_{\text {asleep }}=3.9 \cdot 10^{-8} \mathrm{~kg} \cdot \mathrm{s}^{-1}$, compared to $\beta_{\text {active }}=4.98 \cdot 10^{-8} \mathrm{~kg} \cdot \mathrm{s}^{-1}$ of active moving ones.

Cell immobilization with 2-deoxy- $D$-glucose as glucose substitute is reversible for incubation times smaller than $45 \min (\underline{40})$, whilst the overall cell structure remains unchanged. The observed decrease in end to end distance, given by the major axis length on the other hand, indicates a slight decrease in cell stiffness (41), possibly as a direct effect of 2-deoxy-Dglucose induced lack of adenosine triphosphate ATP. We consider cell immobilization by substitution of glucose as an excellent method for temporary cell immobilization. Cell fixation methods using paraformaldehyde or glutaraldehyde for protein crosslinking induce severe changes in the chemical and hence mechanical properties of cells. Such changes would give rise to artefacts in our measurements due to changes in morphology that are to be avoided. 
With no major changes in cell shape and reversibility of the immobilization method, no major changes in cellular composition are to be expected and the optical forces acting on the immobilized cells should be well comparable to those acting on active cells.

\section{Optical stall forces on trypanosomes}

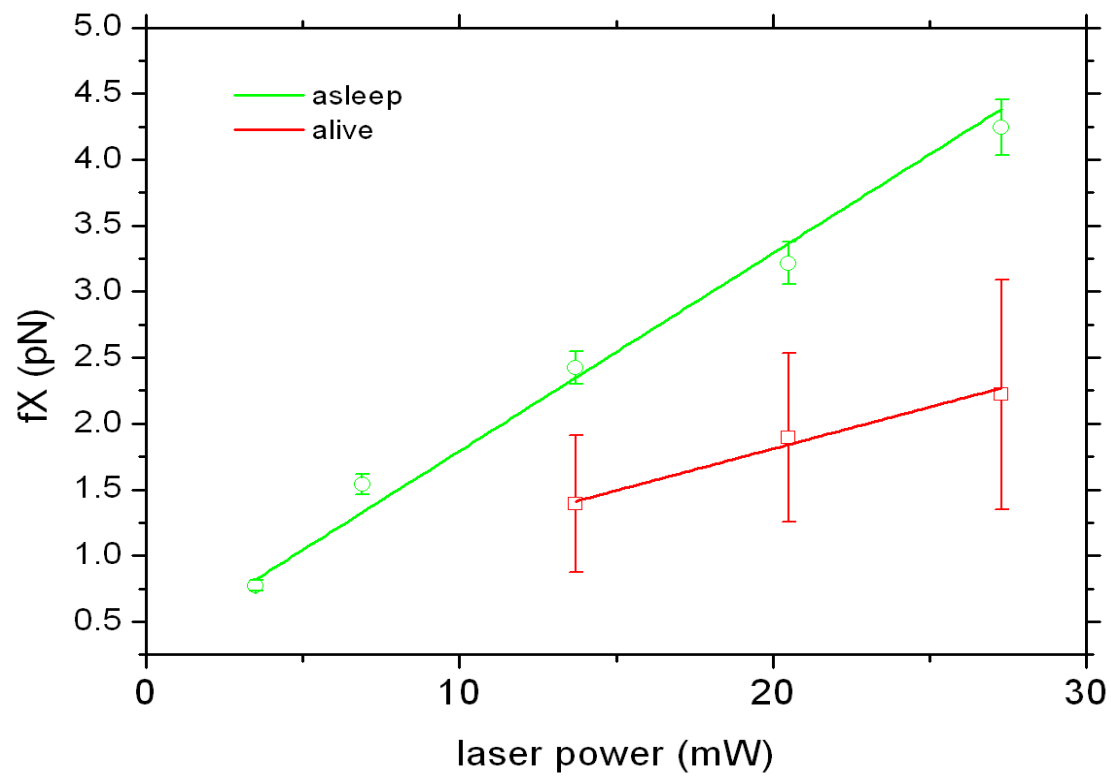

Fig. 3.11: Optical stall forces in TDB medium at $25^{\circ} \mathrm{C}$. Green circles: mean stall force on immobilized trypanosomes; red squares: mean stall force on active trypanosomes; green line: linear fit (slope: 149 $\mathrm{fN} \cdot \mathrm{mW}^{-1}$, interception: $0.28 \mathrm{pN}$ ) ; red line: linear fit (slope: $63 \mathrm{fN} \cdot \mathrm{mW}^{-1}$, interception: $0.55 \mathrm{pN}$ ); green and red bars: error bars $(\sigma)$

Optical stall forces on immobilized $B S F$ trypanosomes have been determined with $F_{S \text { immo }}=$ $149 \mathrm{fN} \cdot \mathrm{mW}^{-1}$ compared to $F_{\text {S active }}=63 \mathrm{fN} \cdot \mathrm{mW}^{-1}$ for active cells.

To determine whether the stall forces are different for immobilized cells, or the propulsion forces of active cells make up for the difference, the propulsion velocities are taken into account. The typical mean velocity for a directional swimmer is given with $v=6 \mu \mathrm{m} \cdot \mathrm{s}^{-1}$ in literature, but velocities can exceed $v=40 \mu \mathrm{ms}^{-1}$ over shorter timescales ( $\left.\underline{41}\right)$. With peak velocities of $40 \mu \mathrm{m} \cdot \mathrm{s}^{-1}$ and a friction coefficient of $\beta=4.98 \cdot 10^{-8} 8 \mathrm{~kg} \cdot \mathrm{s}^{-1}$ an active trypanosome can contribute a drag force of $F_{\text {drag }}=1.9 \mathrm{pN}$ to the actual fluid drag. Adding the measured optical stall force to this stall force we get $F_{S \text { active add }} \cong 4.2 \mathrm{pN}$ at $P=27 \mathrm{~mW}$ laser power, compared to $F_{\text {Simmo }}=4.22 \mathrm{pN}$ observed for immobilized cells. Therefore we conclude that the optical stall force on trypanosomes is almost the same for the immobilized as well as for the active moving cell $\left(F_{\text {ST.b.b }} \approx 150 \mathrm{fN} \cdot \mathrm{mW}^{-1}\right)$. The generation of a propulsion force in the order of $1-2 \mathrm{pN}$ leads to a smaller escape velocity in the case of the moving trypanosomes.

Cell morphology and friction coefficients for active and immobile BSF trypanosomes were determined to be significantly different to the outstretched cell. Based on these results optical 
stall forces on trypanosomes were calibrated in dependency on laser power with a precision of about $5 \%$ (Fig. 3.11, STD given as error bars). A calibrated optical trap facilitates force measurements on the pico-Newton scale. It is now possible to not only to manipulate single cells with sub micrometer precision, but also to analyze their optical properties and the forces they can generate.

\section{REFRACTIVE INDEX OF A TRYPANOSOME}

In order to determine the optical properties of Trypanosoma brucei brucei, we measure the optical stall forces on $D=3 \mu \mathrm{m}$ polystyrene spheres with a refractive index of $n=1.58$ at $\lambda=$ $808 \mathrm{~nm}(\underline{42})$. The relation of optical stall forces between cells and reference bead gives an assumption of the refractive index of the trypanosomes. The optical stall force is given by the gradient force (25) for $F_{\text {gradient }} \gg F_{\text {scatter }}$ with

$$
F_{\text {grad }}=\frac{1}{2} n_{m} \cdot\left(\frac{m^{2}-1}{m^{2}+2}\right) \cdot \nabla \vec{E}^{2}
$$

where $m$ is the effective refractive index defined by

$$
m=\frac{n_{p}}{n_{m}}
$$

with $n_{p}$ the refractive index of the particles and $n_{m}=1.326$, the refractive index of the medium at $\lambda=808 \mathrm{~nm}$ (assuming $n_{T D B} \cong n_{H 20}$ ). The prefactor

$$
\frac{m^{2}-1}{m^{2}+2}
$$

can be approximated by a Taylor expansion of first order, close to 1 by $2 / 3[\mathrm{~m}-1]$.

\section{Prefactor approximation}

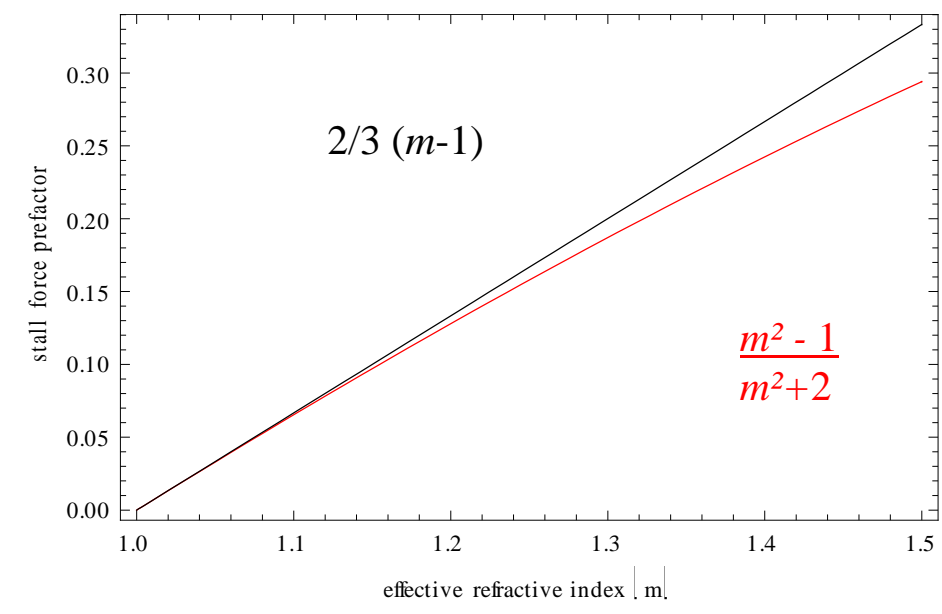

Fig. 3.12: Taylor expansion of the effective refractive index prefactor. Black line: Taylor expansion; red line: prefactor (Eq. X). 
This approach is accurate up to the highest possible effective refractive index of 1.2 (polystyrene to medium), hence the stall force is proportional to $n_{p}-n_{m}$. With

$$
\frac{F_{\text {tryp }}}{F_{\text {bead }}}=\frac{n_{\text {tryp }}-n_{m}}{n_{\text {bead }}-n_{m}}
$$

the refractive index of a trypanosome is given by

$$
n_{\text {Tryp }}=\left[\frac{F_{\text {tryp }}\left(n_{\text {bead }}-n_{\text {medium }}\right)}{F_{\text {bead }}}\right]+n_{\text {medium }}
$$

under the assumption that the effective trapping diameter of the cell is comparable to the reference bead $\left(r_{\text {tryo }} \cong r_{\text {bead }}\right)$.

\section{Optical stall forces on polystyrene spheres}

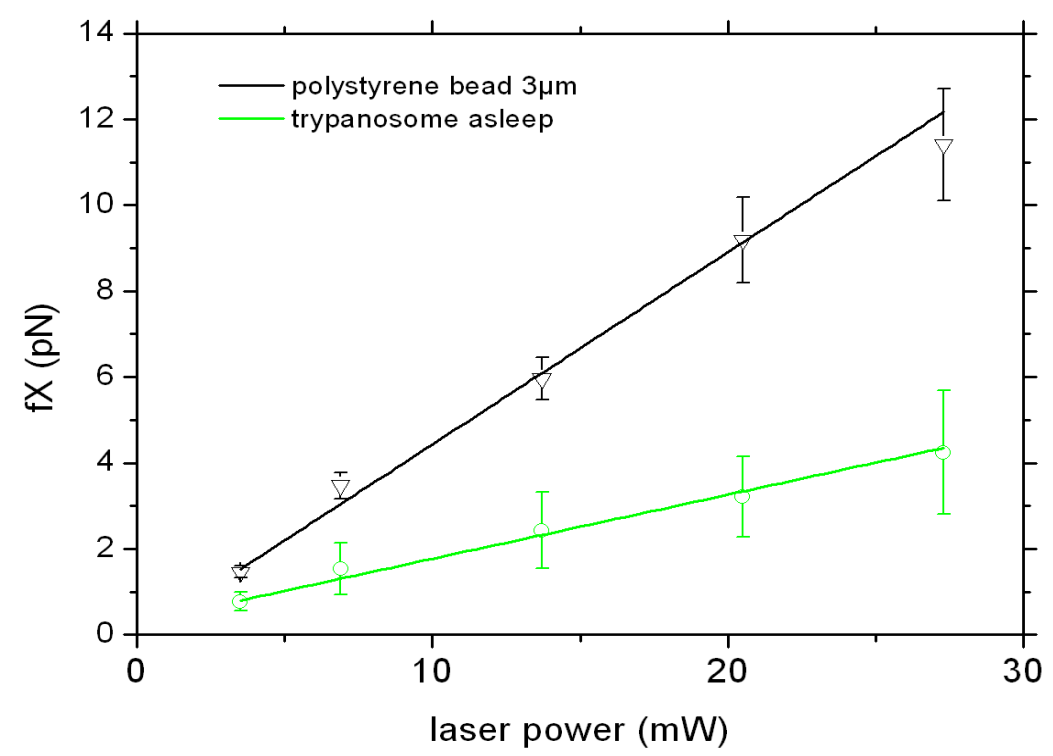

Fig. 3.13: Comparison of optical stall forces on $D=3 \mu \mathrm{m}$ polystyrene spheres and immobilized trypanosomes in TDB medium at $25^{\circ} \mathrm{C}$. Black triangles: mean stall force on polystyrene spheres; green circles: mean stall force on immobilized T. b. brucei; black line: linear fit (slope: $447 \mathrm{fN} \cdot \mathrm{mW}^{-1}$, intercept: $0.28 \mathrm{pN}$ ); green line: linear fit (slope: $149 \mathrm{fN} \cdot \mathrm{mW}^{-1}$, intercept: $0.4 \mathrm{pN}$ ); black and green bars: error bars $(\sigma)$

Optical stall forces on $D=3 \mu \mathrm{m}$ polystyrene spheres have been determined with $F_{S}$ sphere $=$ $447 \mathrm{fN} \cdot \mathrm{mW}^{-1}$, compared to $F_{S}$ tryp $=149 \mathrm{fN} \cdot \mathrm{mW}^{-1}$ for immobilized $B S F$ trypanosomes. The refractive index of T.b. brucei calculated from these measurements is $n_{\text {tryp }}=1.435 \pm 0.012$.

The measurements are in good agreement with published data for the refractive indices of eukaryote cells, ranging from $n_{b i o}=1.33-1.45(\underline{43})$. However no data has been found on refractive indices of trypanosomes and we consider the obtained values as an extension of the present spectrum. 


\section{TRAPPING LOCI OF TRYPANOSOMES}

\section{INTRODUCTION}

Biological matter is often inhomogeneous in composition and complex of shape. Therefore it is not surprising that optical forces on a trapped cell vary over position. In our experiments we see trypanosomes moving through a weak optical potential like a piece of thread, sliding back and forth through the eye of a needle. According to the central limit theorem, the trapping locus of a rod shaped object (with $L_{\text {rod }}>>\theta_{\text {beamwaist }}$ and $D_{\text {rod }} \gg \theta_{\text {beamwaist }}$ ), solely driven by Brownian motion, should be Gaussian distributed (with $F_{\text {gradient }} \gg F_{\text {scatter }}$ ). Any deviation from such a distribution is either due to inhomogeneities in structure, or directed movements of the cell. The trapping loci of Trypanosoma brucei brucei are quantified in the following chapter revealing interesting features of both cellular structure and motility of the cell.

\section{EXPERIMENTS}

Trapping loci of trypanosomes were analyzed in a microfluidic stop flow PDMS device to prevent from outer disturbances, induced by external fluid. All cells were trapped $25 \mu \mathrm{m}$ below the cover slip surface, in a chamber of $65 \mu \mathrm{m}$ in height and at a distance larger than 50 $\mu \mathrm{m}$ to each side wall to avoid hydrodynamic interaction with those.

\section{Preparation OF CELlS}

Trypanosoma brucei brucei were prepared as described before. A microfluidic stop flow device is used to exclude fluid flow disturbances using $1 \mathrm{~mL}$ plastic syringes. Trapping loci were recorded at a framerate of $100 \mathrm{fps}$ over $30 \mathrm{~s}$ for different lasing intensities.

\section{IMAGE PROCESSING}

The center of mass of trypanosomes was obtained of the images by a combination of background creation and subtraction, stringent thresholding, denoising and strong dilation of the objects which results in a reduction of detail, but a gain in signal to noise ratio, most suitable for the particular analysis (chapter 2.3).

Manual tracing of the posterior and anterior cell was conducted for high resolution positioning and confirmation of automated image processing. 


\section{RESULTS AND DISCUSSION}

\section{TRAPPING LOCI OF TRYPANOSOMES}

A living trypanosome, drawn into an optical trap, maintains mobile, because the focal volume of an optical trap is very small in comparison to the cell volume $V_{\text {trap }}=0.03 \mu \mathrm{m}^{3}<<V_{\text {tryp }}=$ $100 \mu \mathrm{m}^{3}$. Whilst mobility is limited, motility remains almost free, much like a dog on a leash.

High speed imaging techniques were integrated into the optical trapping microscope setup. The combination of both allows high resolution imaging of very fast but tiny movements which is necessary to examine the rapid and complex motility of trypanosomes. Together with automated image processing routines, it is possible to analyze trypanosome motility and positioning within the optical trap in high detail and quantity.

The planar projection of a living trypanosome, recorded at $100 \mathrm{fps}$ within an optical trap, is shown in figure $3.14 \mathrm{~b}$. The red line shows the cell shape, as detected with our routines, used for calculating the center of mass. The green line displays an elliptical fit to the shape, giving both, the width and length of the cellular expansion. The data is plotted as displacement of the center of mass over time in figure $3.14 \mathrm{~b}$ (red line), where the length of the major axis is given by the green line.

\section{Trapped trypanosome trace and shape}

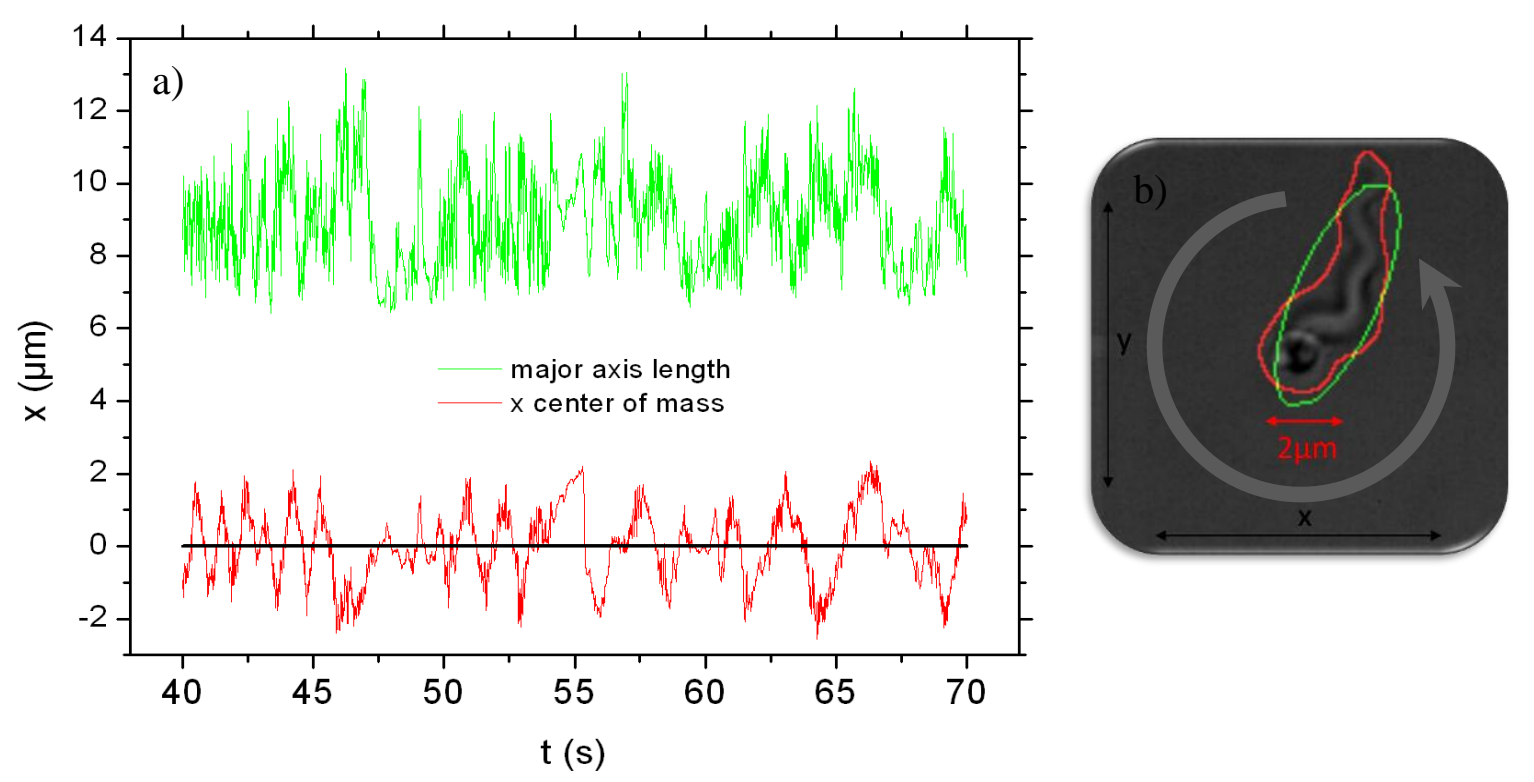

Fig. 3.14: Shape descriptors and trace of a trypanosome. Right figure a): trapped trypanosome rotating counterclockwise (optical trap is always in the center). Red line: detected cell shape; green line: elliptical fit to shape. Left figure red line: com displacement in $\mathrm{x}$ axis direction; green line: length of the major axis. 
The cell rotation indicated in figure $3.14 \mathrm{~b}$ is described in high detail by the com trace given in figure 3.14a. In combination with the elliptic shape descriptors, it is now possible to quantify both the positioning of the cell within the optical trap, as well as the motility of the cell.

Two very important technical aspects arise from this method: 1 . The Motility of a single cell can be recorded in high spatiotemporal resolution over long time series. This is usually in contradiction to the small field of view of high resolution optics. Rapid moving cells tend to leave the observed area as soon as possible (personal experience) 2. The rotational aspect of trypanosome motility is much more prominent if seen from the axis of revelation, but not from the side. Using optical trapping on trypanosomes you basically gain an additional point of view, at frame rates and observation times only limited by camera $(<20000 \mathrm{fps})$ and the given storage capacity.

Active cell motility results in dynamic displacement within the trap, giving both information about the cellular structure (44) and the motility of the cell. With the center of mass distance to the trap center and the length of the major cell axis (Fig. 3.14), the trapping locus in relation to the cells contour, can be calculated. Subtraction of $d X$ from the actual half length of the major axis and relation to an outstretched cell contour of $20 \mu \mathrm{m}$ length, gives the distance of the trapping locus relative to the cell center of mass.

\section{Trypanosome trapping topography}
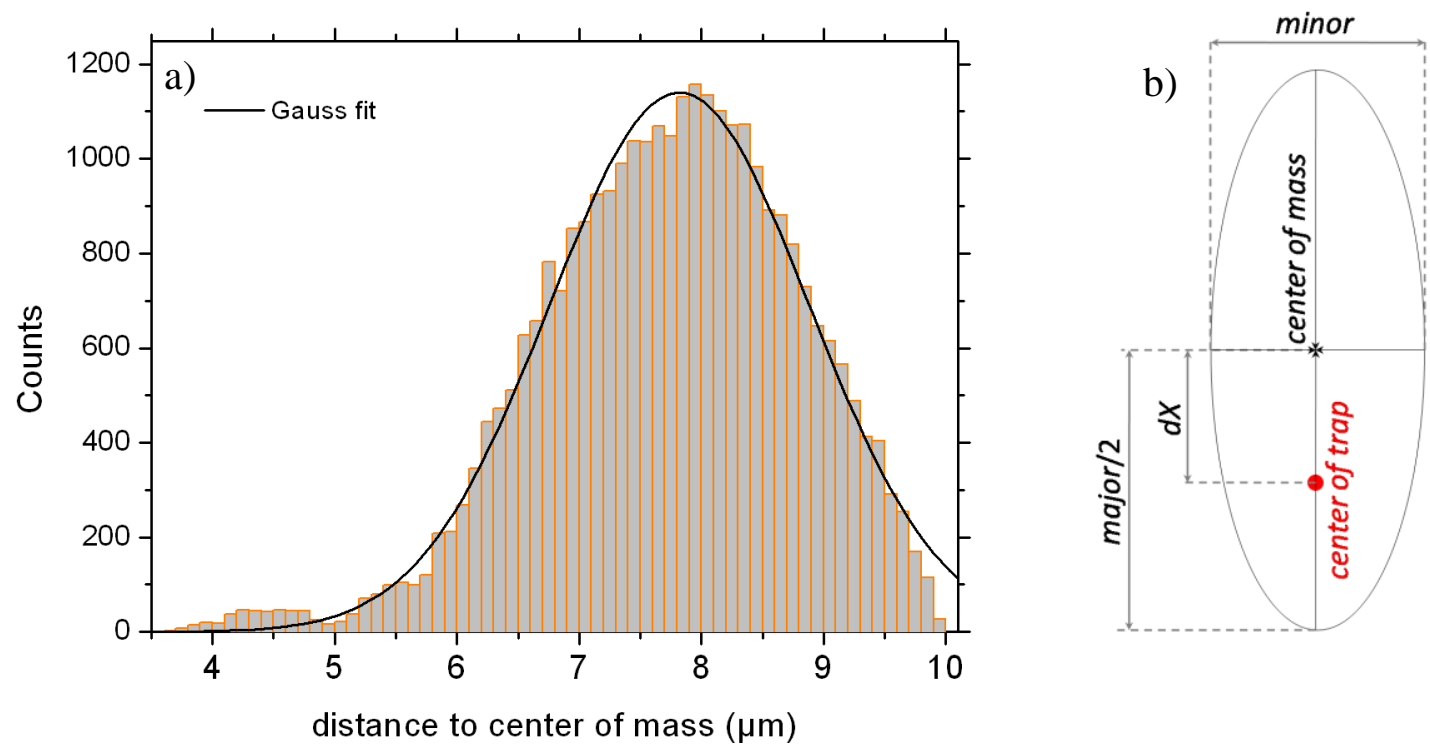

Fig. 3.15: Histogram of trapping loci distance to the cell center of mass. Gray bars: distance counts; black line: Gaussian fit $(\mathrm{c}=7.8 \mu \mathrm{m}, \sigma=2.4 \mu \mathrm{m})$; gray arrow: second peak; $\mathrm{b})$ : sketch of geometric features used for calculation of distance of the trapping locus relative to the cell center. 
With no net direction in its motility and a homogenous cellular structure, the mean trapping locus should be in the center (central limit theorem, $\underline{45}$ ). With a homogeneous structure of the cell, but a net direction of motility, the peak should be at the very tip of the cell (opposite to propagation direction).

Instead the mean trapping locus is located at a distance along the contour of about $7.8 \mu \mathrm{m}$ to the geometric center of mass and about $2.2 \mu \mathrm{m}$ away from the distal end ("head"). This indicates a structure within the cell that is favourable for optical trapping.

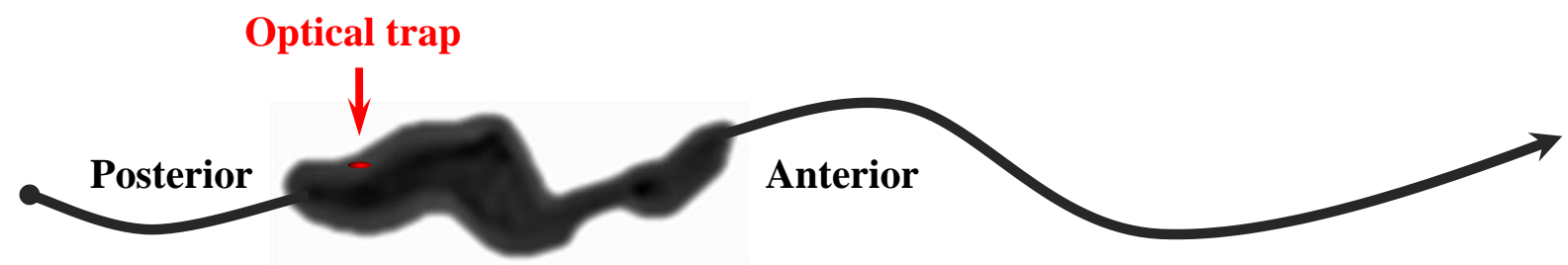

Fig. 3.16: Illustration of the mean trapping locus of a propagating trypanosome close to the flagellar pocket

The trapping locus at a distance of $7.8 \mu \mathrm{m}$ to the cell center coincides with the localization of the flagellar pocket, located in the posterior part of the cell (Fig. 3.16, 16). In order to quantitatively distinguish, whether the mean trapping position is actually located close to the flagellar pocket, or somewhere within the anterior part, two different trypanosomes were manually traced by a computer aided procedure. One cell executes tumbling motion, whilst the other executes running motion.

Indeed, the analysis of those traces reveals a very detailed description, not only of the absolute trapping locus distribution, but also of the cell motility itself (Fig. 3.17). A tumbling cell is sliding back and forth through the optical potential and there is no regular pattern to be found in the path of the cell poles. A "running cell" (which is rotating if trapped) instead, shows a clear tendency of a posterior end to be close to the center, surrounded by the anterior pole due to the rotational motility pattern, as indicated in figure $3.17 \mathrm{a}, \mathrm{b}$ and described in detail in chapter (4). 

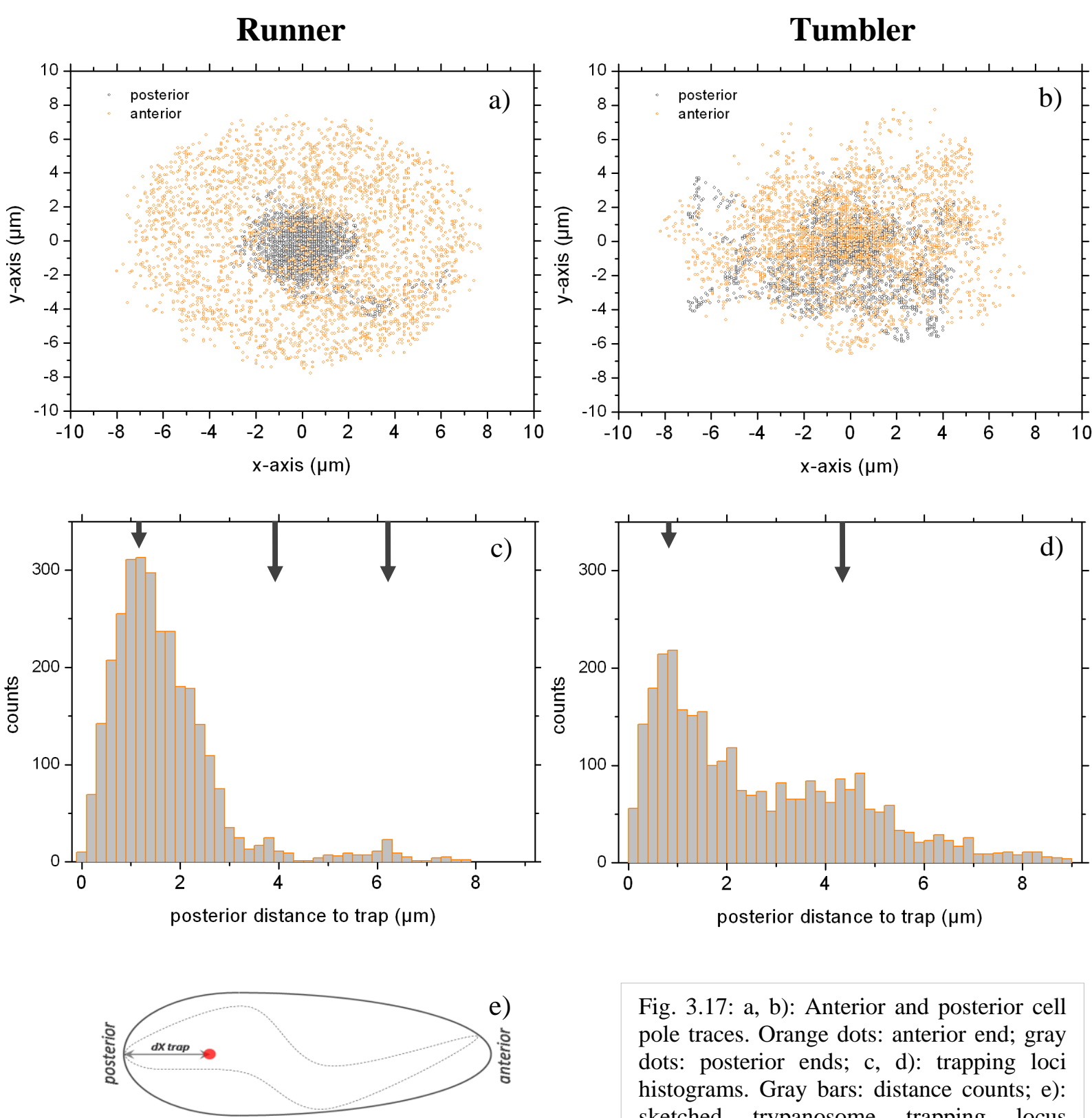

Fig. 3.17: a, b): Anterior and posterior cell pole traces. Orange dots: anterior end; gray dots: posterior ends; c, d): trapping loci histograms. Gray bars: distance counts; e): sketched trypanosome trapping locus determination.

It has been shown that trypanosome cell stiffness varies over the population and is related to cell motility and the posterior end always moves much slower than the anterior (S. Uppaluri, in review). Given the posterior part to be less flexible, the trapping locus is defined by the posterior distance to the trap. As the cell pole traces indicate, the distance histograms clearly show that the trapping locus of a running cell is much more localized than of a tumbling cell, but shares the same features (Fig. 3.17c, d arrows). Most importantly the mean trapping position is actually located in the posterior part of the cell and roughly $2 \mu \mathrm{m}$ away from the very tip. With respect to the quantitative data obtained by automated image processing (Fig. 3.17e), these results clearly show a favourable trapping locus in Trypanosoma brucei brucei, close to the position of the flagellar pocket. 
As organelles vary in function, they also vary in their interaction with light (푸) and represent irregularities in the trapping landscape of trypanosomes. The question is which organelle or structural feature of the cell interacts stronger with laser light, in comparison to the rest of the cell - which is the organelle with the highest index of refraction?
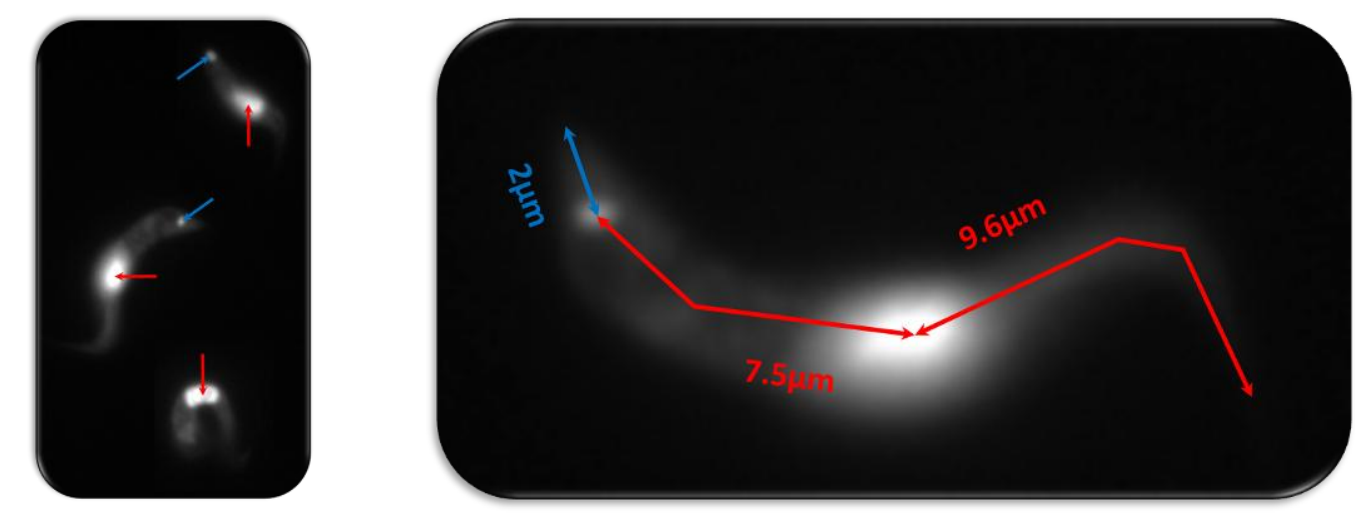

Fig. 3.18: EtBr DNA stain of Trypanosoma brucei brucei. The red arrows indicate the position of the nucleus while the blue arrows indicate the position of the kinetoplast (chapter X).

Both kinetoplast and nucleus are mainly comprised of DNA and can be visualized by ethidium bromide stains in fluorescence microscopy. Analysis of the images gives the position of the kinetoplast $2 \mu \mathrm{m}$ away from the posterior tip of the cell (Fig. 3.18) which corresponds to the mean trapping locus. DNA however, does not contribute a significant change to the refractive index (느), since the nucleus does not colocalize with a preferred trapping locus.

The major contribution to higher indices of refraction is provided by cellular proteins due to their high polarizability (47). A particular high index of refraction $(n=2.90)$ has been found for $\alpha$ and $\beta$ tubulin (47). Interestingly, the tubulin concentration in trypanosomes is very high, because, unlike other cells, the trypanosome cell membrane is structurally supported by a tubulin network, instead of an actin cortex. This gives support to the high refractive index we have measured, compared to eukaryotic cells with actin cortices (43). Tubulin is also the major component of flagellar proteins, with high concentration in the basal bodies which are tightly interconnected with the kinetoplast. The basal bodies represent the microtubule organization center in trypanosomes and anchor the flagellar axoneme to the cytoskeleton (chapter 1, $\underline{48}$ ).

With respect to the tubulin concentration, we assume that the flagellum represents a major contribution to the high refractive index of the cells, with a maximum located at the flagellar pocket as we see it in our measurements. 
Another feature in the trapping landscape of trypanosomes is the existence of additional trapping peaks, located about 4 and $6 \mu \mathrm{m}$ away from the posterior tip (Fig. 3.17c, d, gray arrows). We consider these additional morphological features as curvatures in cell shape. Moving through the optical potential, the cell has to follow its shape but any movement in $z-$ axis direction will delay the lateral displacements we observe in planar projection (Fig. 3.19). This is directly seen in the tapping locus histogram as an increase in abidance probability at the according positions.

\section{Projection of cell morphology and motility}

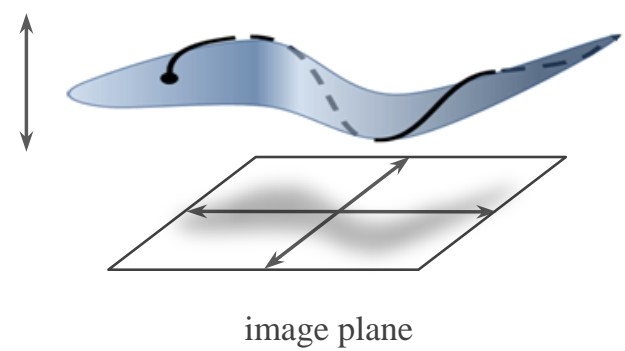

Fig. 3.19: Sketch of a trypanosome undergoing z-axis displacement due to the cell morphology

Although trypanosome propagation is mediated by dynamic reshaping of the cell, these observations indicate a higher order structure, either imprinted onto the general morphology, or periodically reoccurring at well defined positions. Further optical trapping experiments, including organelle labeling, will help to understand the dynamic morphology and hence the motility of trypanosomes.

In order to quantify the optical stall forces in relation to the trapping loci, the stall forces on immobilized trypanosomes were analyzed in dependence of the trapping locus. The trapping loci were classified as posterior, anterior and flagellar pocket, where the criterion for classification was the locus at which a cell finally got dragged out of the trap. The optical stall forces measured in this experiment establish a consistent relation to the trapping locus. 


\section{Optical stall forces on different trapping loci}

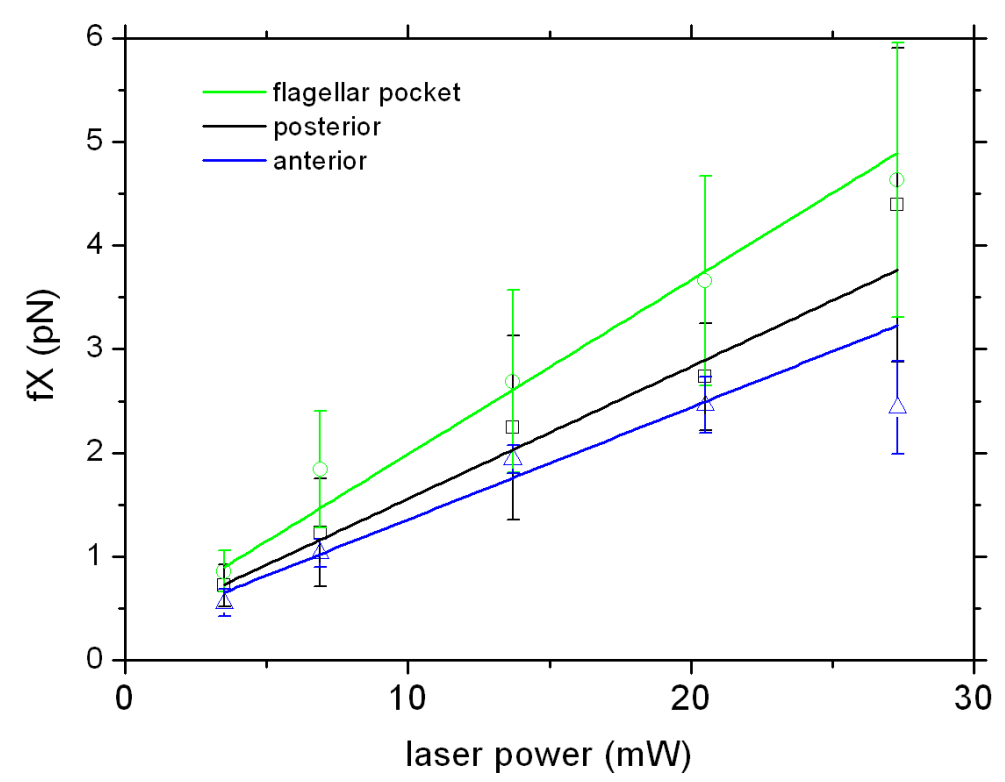

Fig. 3.20: Optical stall forces on posterior, anterior and flagellar pocket in TDB medium at $25^{\circ} \mathrm{C}$. Green circles: mean stall force on flagellar pocket; black squares: mean stall force on posterior; blue triangles: mean stall force on anterior green line: linear fit (slope: $168 \mathrm{fN} \cdot \mathrm{mW}^{-1}$, interception: $0.31 \mathrm{pN}$ ); black line: linear fit (slope: $127 \mathrm{fN} \cdot \mathrm{mW}^{-1}$, interception: $0.29 \mathrm{pN}$ ); blue line: linear fit (slope: $108 \mathrm{fN} \cdot \mathrm{mW}^{-1}$, interception: $0.27 \mathrm{pN}$ ); green black and blue bars: error bars $(\sigma)$

Optical stall forces at the flagellar pocket are significantly higher compared to the distal ends, with $F_{S f p}=168 \mathrm{fN} \cdot \mathrm{mW}^{-1}$ at the flagellar pocket, compared to $F_{S \text { post }}=127 \mathrm{fN} \cdot \mathrm{mW}^{-1}$ at the posterior tip and $F_{S \text { ant }}=108 \mathrm{fN} \cdot \mathrm{mW}^{-1}$ at the anterior tip of the cell.

These measurements confirm the most frequent trapping locus to be also the strongest trapping locus which we identify as the origin of the flagellum. The weaker trapping forces at the anterior tip may be explained by the small diameter of the flagellum, compensating for the high tubulin concentration. With an approximate volume of the sole flagellum of only $V_{\text {flag }}=5.0 * 10^{-20} \mathrm{~m}^{3}(r=200 \mathrm{~nm}(\underline{16}), l=$ beam waist diameter $=400 \mathrm{~nm})$ which is smaller than the theoretical trapping volume of $9.7 * 10^{-20} \mathrm{~m}^{3}(l=400 \mathrm{~nm}$, focal length $=600 \mathrm{~nm})$, the optical potential is not saturated hence trapping forces are weaker.

Optical trapping topography revealed new optical and structural features of Trypanosoma brucei brucei and provides background for further biophysical studies on cell motility and trypanosome motility, as described in the next chapters. 


\section{DEPENDENCY ON LASER POWER}

The stiffness of optical traps is measured by the force needed to displace an optically confined particle over a certain distance. It increases linearly with laser power and is also approximated to be constant within a range of one particle radius. In other words, the Brownian motion of a particle should decrease with increasing laser power.

For very big particles with $r<<\lambda$, the stiffness decreases, because neither volume nor the geometry of the particle within the optical trapping volume changes significantly with particle displacement. In the case of trypanosomes however, the situation is more difficult: As we could show the trapping forces vary with position (Fig. 3.20) and the cells are actively moving. Recording the center of mass displacement of trypanosomes over laser power, we actually see an increase in the deviation of displacement with increasing laser power.

\section{Trap stiffness}
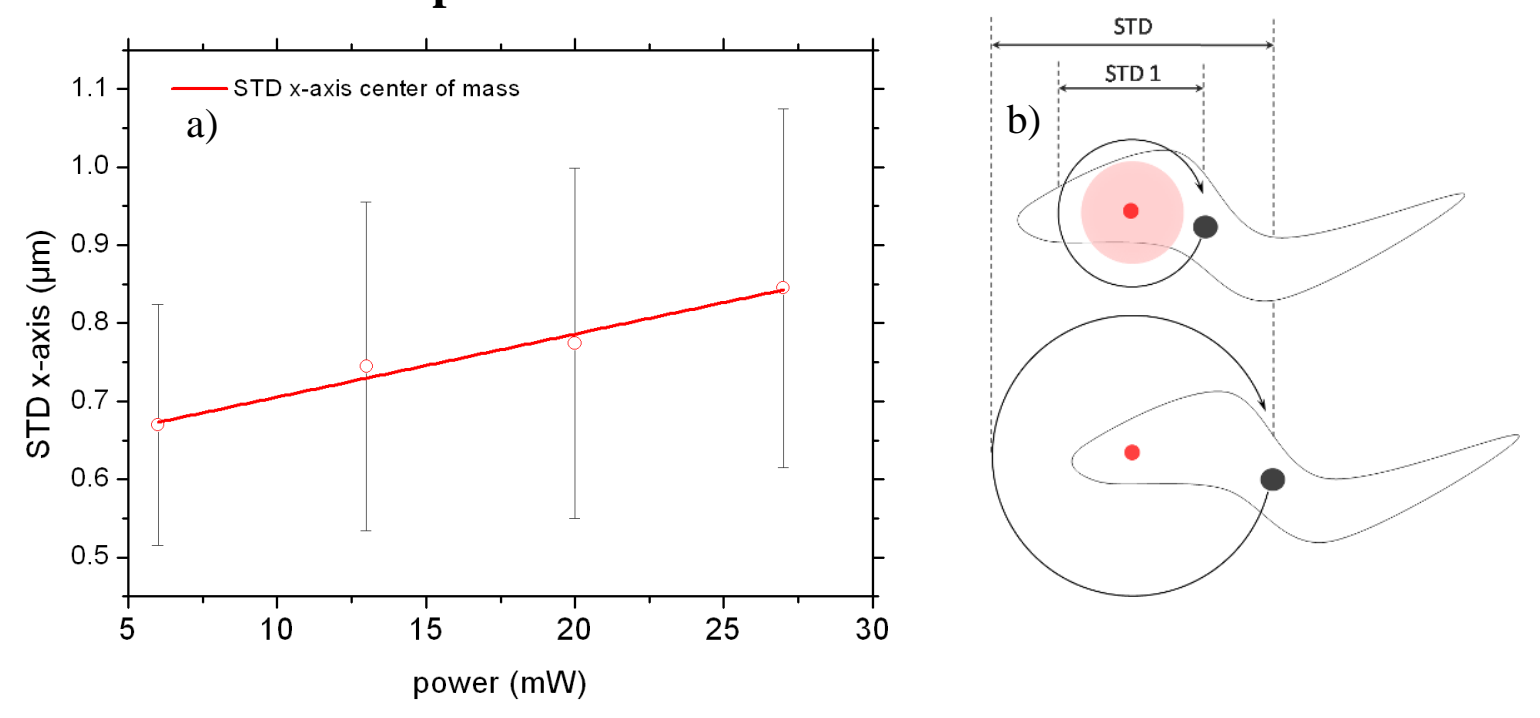

Fig. 3.21: a) Center of mass deviation in $x$-axis direction of trypanosomes over laser power in TDB medium at $25^{\circ} \mathrm{C}$. Red circles: mean com deviation over laser power; red line: linear fit (slope: $8.1 \mathrm{~nm} \cdot \mathrm{mW}^{-1}$, interception: $0.63 \mu \mathrm{m})$; gray bars: error bars $(\sigma)$. b) Com deviation changes with trapping locus stiffness: $S T D 1<S T D$

This finding would indicate a decrease in trapping stiffness with increasing laser power, but taking into account motility, shape and structure of the cell, the conclusion is different. In a population of trypanosomes there is always a certain amount of running cells, so that there is always a mean net progress, resulting in a mean net rotation in optical confinements. Taking into account that the most frequent trapping locus is located close to the flagellar pocket which is not the center of mass, that center of mass undergoes a rotational motion too. Reduced trap stiffness results in a broader trapping locus distribution towards the center of mass (Fig. 3.21). In return the mean center of mass position is more often close to the trapping center, hence the center of mass standard deviation decreases. 


\section{TRYPANOSOME FORCES}

\section{INTRODUCTION}

Probably, the biggest advantage of optical trapping is its weakness. With a well calibrated trap, it is possible to measure forces on the pico Newton scale and with nanometer precision, the forces and movements of single molecules and cells. We calibrated the optical stall forces on trypanosomes in order to measure the propagation forces of $B S F$ trypanosomes in medium. By introduction of a second optical trap, we were able to also measure flagellar beat forces.

\section{EXPERIMENTS}

The laser power at an event of trypanosome trap escape was recorded for $n=30$ cells and the propulsion forces were calculated by the experimental values of chapter 3 .

Flagellar beating forces were analyzed in a dual trap configuration, where the beat displacement patterns was recorded over time and analyzed with calibration data obtained for the setup situation.

\section{Preparation OF CELlS AND MiCROFluidic DeVices}

Trypanosoma brucei brucei were prepared as described before. Optical trapping experiments were conducted in a mircofluidic stop flow device.

\section{Propulsion force MEASUREMENT}

The cells were initially trapped $25 \mu \mathrm{m}$ below the coverslip surface at $20 \mathrm{~mW}$ laser power which was subsequently reduced in $1 \mathrm{~mW}$ steps every $5 \mathrm{~s}$. Laser power and cell cycle were recorded at the event of trap escape.

\section{BEAT FORCE MEASUREMENTS}

Trypanosomes were trapped $10 \mu \mathrm{m}$ below the coverslip surface for an increase of the trapping stiffness. A polystyrene bead of $r=1.553 \mu \mathrm{m}$ was trapped in a secondary trap, $15 \mu \mathrm{m}$ below the coverslip surface, at a lateral distance of $3 \mu \mathrm{m}$ to the primary trap. The event of beat contact was recorded at a framerate of 2000 fps. The secondary trap was calibrated for a position $15 \mu \mathrm{m}$ below the coverslip surface by the Stokes drag method. 


\section{RESULTS AND DISCUSSION}

\section{PROPUlSION FORCES OF TRYPANOSOMES}

Proliferating trypanosomes duplicate by fission. The process of duplication is accompanied by the growth of a second flagellum and the cells exist as duplets for a significant part of their cell cycle (16). Despite the importance of this stage, we did not find any literature analyzing the impact of the second flagellum on cell motility. The propulsion forces of single and double cells have been measured by comparing the optical stall forces the different cells were able to escape.

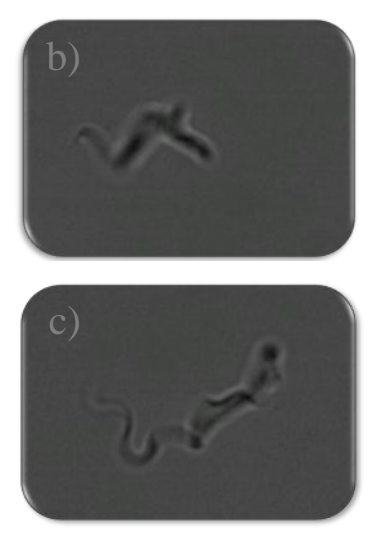

\section{Trypanosome propulsion forces}

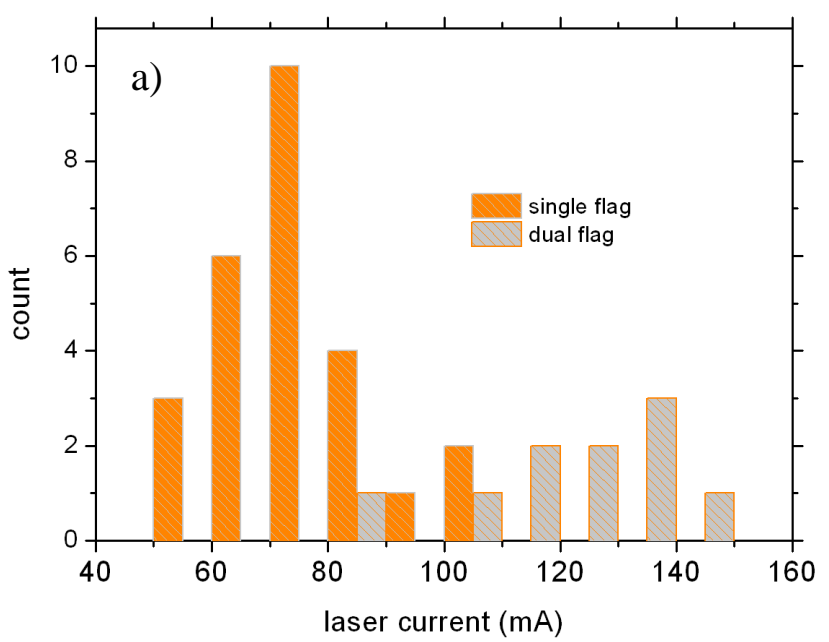

Fig. 3.22: a) Histogram of $n=26$ single cells and $n=10$ cell duplets escape currents. b) single cell; c) cell duplet; Both cells a), b) were trapped at an optical power of $20 \mathrm{~mW}$.

The propulsion forces were measured for 26 single cells (orange bars) and 10 cell duplets (gray bars). The resulting histogram of recorded laser currents at the event of cell escape clearly shows different distributions for the two kinds of cells. The escape force was calculated by the given laser current and a hydrodynamic friction coefficient of $\beta_{\text {active }}=$ $4.98 \cdot 10^{-8} \mathrm{~g} \cdot \mathrm{s}^{-1}$ for living cells. We found single cells to propel with a mean force of $F$ single $=$ $0.83 \mathrm{pN}$, while cell duplets propel with almost exactly twice the force of $F$ duplet $=1.78 \mathrm{pN}$. We understand that cells duplets which have divided, can eventually propel into the same direction at a time and thereby add their propulsion forces. This is a very interesting finding, showing that cells which have already grown a second flagellum, but have not cleaved yet, might swim twice as fast, or produce twice as much force as single cells. This cell cycle stage might indeed represent an adaption to specific needs in fast propagation as well as tissue invasion. The spread of propulsion force can be explained by the different progression in cell cycle i.e. growth of flagellum, for the cell populations examined, since morphological distinction of these sub-stages is not possible by visual inspection without using specific cell cycle tags. Vice versa measurement of propulsion forces gives information of the cell cycle. 


\section{FLAGELLAR BEAT STRENGTH}

Using two optical traps simultaneously, it is possible to measure the force cells can act on other objects like blood cells, endothelial cells or components of the extracellular matrix. With the posterior part of a trypanosome $\left(\beta_{\text {active }}=4.98 \cdot 10^{-8} \mathrm{~kg} \cdot \mathrm{s}^{-1}\right)$ quasi fixed in a strong optical trap $\left(F_{\text {stall tryp }}=9 \mathrm{pN}\right)$, the forces exposed by the anterior part, beating against a small polystyrene bead $\left(\beta_{\text {active }}=2.8 \cdot 10^{-8} \mathrm{~kg} \cdot \mathrm{s}^{-1}\right)$ and trapped in weak optical trap $\left(F_{\text {stall bead }}=3.7 \mathrm{pN}\right)$ were recorded by high speed imaging $(f=1000 \mathrm{~Hz})$ of bead displacement.

Trypanosome football

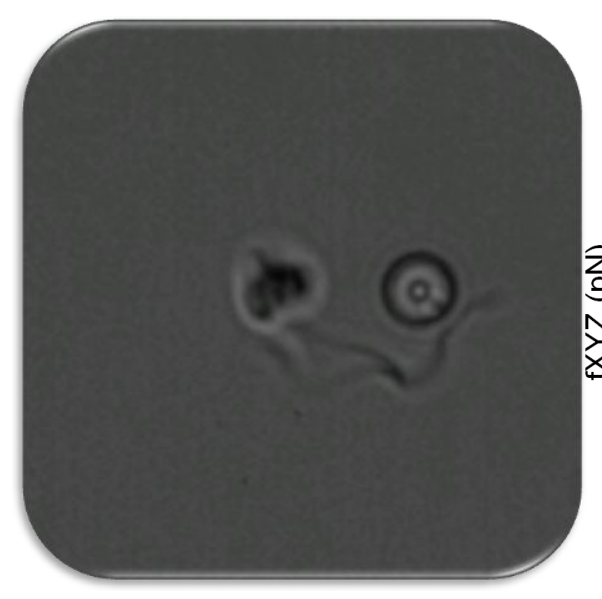

Force profile

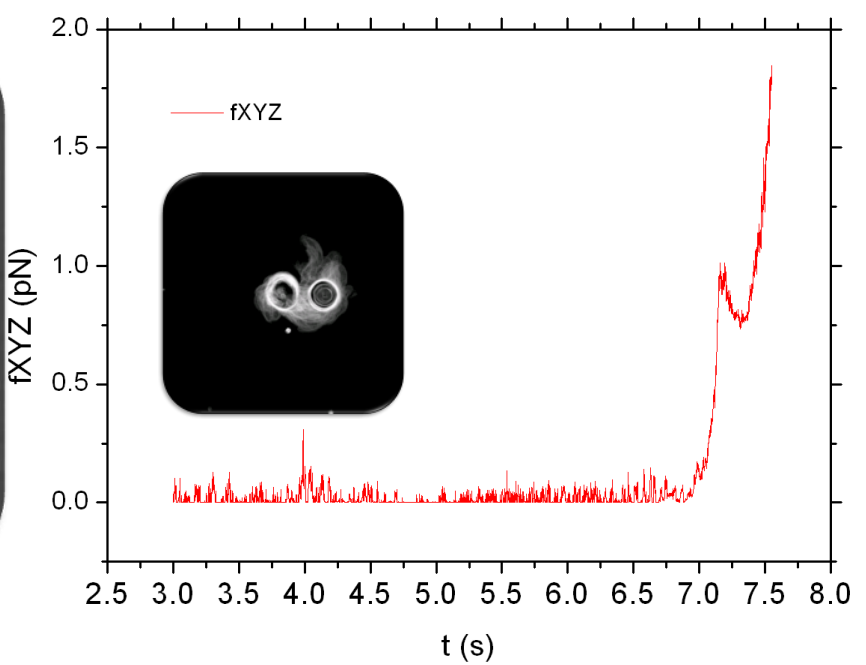

Fig. 3.23: a) Trypanosome trapped at $P=27 \mathrm{~mW} 10 \mu \mathrm{m}$ below the coverslip surface beating against a $D=3$ $\mu \mathrm{m}$ polystyrene bead trapped at $P=13 \mathrm{~mW}$ power $15 \mu \mathrm{m}$ below the coverslip surface. b) Sum of the forces acting on the bead in $\mathrm{XYZ}$ axis directions over time.

Both optical stall force and the stiffness of the trap were recorded immediately after the experiment. The maximum optical stall on the $D=3 \mu \mathrm{m}$ polystyrene bead was determined with $F_{\text {stall }}=3.75 \mathrm{pN}( \pm 0.25 \mathrm{pN}) 15 \mu \mathrm{m}$ below the coverslip. The stiffness of the trap was determined by recording the displacement power spectrum at a framerate of $f=20 \mathrm{kHz}$ for all three axes, as described in chapter 2. The stiffness values obtained for each axis were $F_{x}=$ $17.3 \mathrm{pN} \cdot \mu \mathrm{m}^{-1}, F_{Y}=14.0 \mathrm{pN} \cdot \mu \mathrm{m}^{-1}$ and $F_{z}=5.5 \mathrm{pN} \cdot \mu \mathrm{m}^{-1}$. The trypanosome shown in figure 3.23a was rotating counter clockwise, while the event of bead contact is given by the force plot in figure $3.23 \mathrm{~b}$. The anterior part of the cell passes by the bead at $t=4 \mathrm{~s}$, displacing it slightly, but at $t=7 \mathrm{~s}$ it hits the bead and rotation is halted for about $0.5 \mathrm{~s}$. After several flagellar cycles however, the bead is finally kicked out of the optical potential, overcoming the maximum stall force of $3.75 \mathrm{pN}$. As for this experiment, we estimate that trypanosomes can generate forces $\geq 5 \mathrm{pN}$ with their anterior end. The use of high viscous media or stronger lasers is necessary to really determine the maximum force which can generated at the anterior end. 


\section{PHOTOTOXICITY OF OPTICAL TRAPPING}

\section{INTRODUCTION}

Optical trapping provides the unique potential of contact free and hence sterile micromanipulation of living organisms. Still the interaction of light with matter always results in absorption of single or several photons, namely two photons, which can heat up the sample or induce toxic chemical reactions. The likeliness of photon absorption is dependent on the wavelength and molecules exposed, thus the wavelength should be chosen for the least absorption in the manipulated object of interest. The following experiments have been conducted in order to quantify phototoxic effects on trypanosome vitality.

\section{EXPERIMENT}

Phototoxic effects of the $808 \mathrm{~nm}$ trapping laser on Trypanosoma brucei brucei have been analyzed in long time series experiments. The experiments were conducted with freshly thawed cells that were trapped at different lasing intensities, while motility was recorded as a measure of their vitality.

\section{PREPARATION OF CELLS AND MiCROFLUIDIC DEVICES}

Trypanosoma brucei brucei were prepared as described before. Optical trapping experiments were conducted in a mircofluidic stop flow device. All cells were trapped $25 \mu \mathrm{m}$ below the cover slip surface and their motility was recorded at a framerate of $20 \mathrm{fps}$ for $60 \mathrm{~min}$ each. The sample was illuminated with a 4 W, 370 lumen CREE XPGWHT-L1-1T-R5 LED light source.

\section{IMAGE PROCESSING}

The center of mass displacement as the measure of cell motility, was derived as described in chapter 2. 


\section{RESULTS AND DISCUSSION}

\section{PHOTOTOXICITY OF OPTICAL TRAPPING}

An effective optical trapping setup for micromanipulation of living cells should have little phototoxic effects on the exposed organisms. To quantify phototoxic effect of $808 \mathrm{~nm}$ laser light on trypanosomes the measurement of cell motility has proven to be a good measure for cell vitality.

Long time measurements of trypanosomes, trapped at a lasing power of $27 \mathrm{~mW}$, show vital cells after trapping periods even longer than $60 \mathrm{~min}$. In the experiment, five cells were trapped at four different laser powers and the overall motility, as a sum of the absolute center of mass displacement, is plotted over time in figure 3.24.

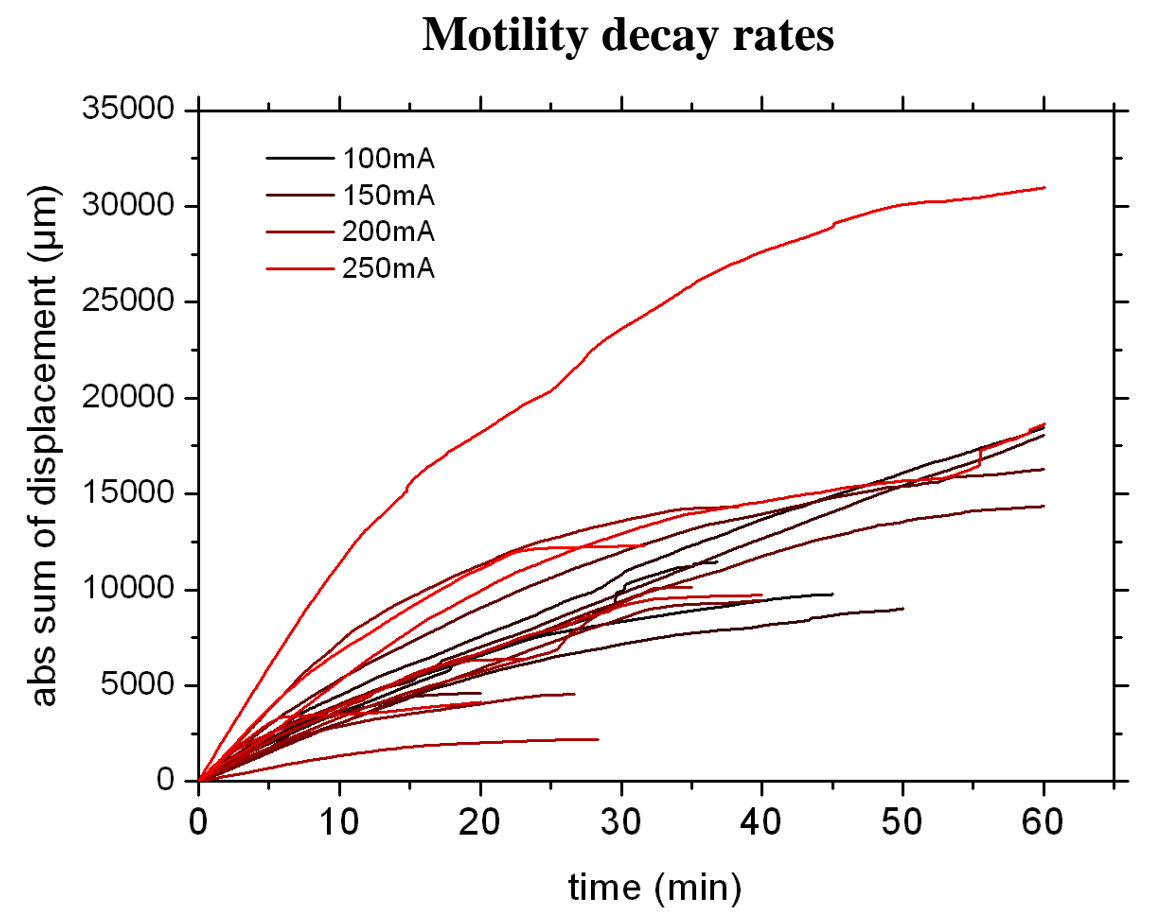

Fig. 3.24: Sum of $\mathrm{x}$-axis displacements over time. For each laser power five cells were traced over a period from 20 to $60 \mathrm{~min}$ according to the time until active motility had vanished.

The overall motility of the cells analysed varies very much, as to be expected from a population of trypanosomes. The cells die sooner or later, depending on the laser power they have been exposed to. Since two photon absorption is a nonlinear third order process, phototoxic effects are expected to scale exponentially with laser intensity (쑤). With the motility decay as a rate of vitality, the sum of absolute cell displacement was fitted by an exponential decay function to calculate the half-life time of all examined cells. With a maximum laser power of $27 \mathrm{~mW}$ at the sample, we find a mean half-life time larger than 15 min. The first conclusion we can draw is that for all optical trapping measurements we carried 
out on trypanosomes, photodamage can be neglected, since no trapping event lasted longer than 6 minutes including cell transportation.

Photodamage on biomatter is thought to be mainly caused by simultaneous two photon absorption, creating toxic radicals within the cell (44). Sample heating on the other hand is considered to be of minor importance ( $\underline{50})$. Heating of biomatter by a $1064 \mathrm{~nm}$ trapping laser, has been measured experimentally with approximately $1.45 \mathrm{~K}$ for every $100 \mathrm{~mW}$ of laser power at the sample. Heating from $830 \mathrm{~nm}$ laser is expected to be $5 \mathrm{x}$ lower $(\underline{51})$.

Based on these findings, a lasing wavelength of $808 \mathrm{~nm}$ has been chosen for the experimental setup, where an expected sample heating smaller than $0.1 \mathrm{~K}$ can be considered as negligible.
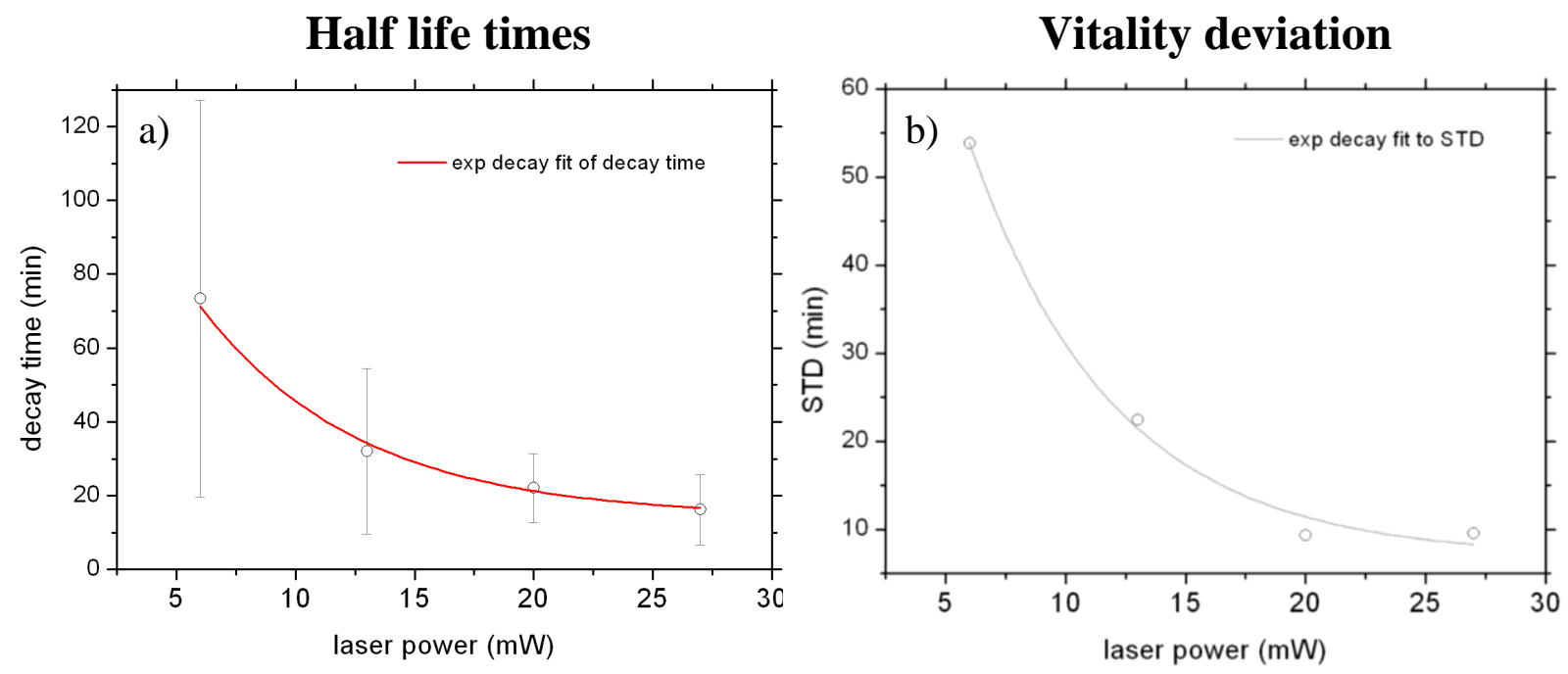

Fig. 3.25: The absolute sum of $\mathrm{x}$-axis displacements is plotted over time. For each laser power five cells were traced over a period from 20 to 60 min according until active motility vanished as seen by visual inspection.

The exponential motility decay rates observed for single cells over time (Fig. 3.25) do not only coincide with an exponential decay of the half life time over laser power (Fig. 3.25 a), but also with the exponential decay we observe for the standard deviation in half life times over laser power (Fig. 3.25 b). In combination, these results indicate that cell death is very likely to be caused by two photon absorption.

The phototoxic effects of optical trapping at a wavelength of $\lambda=808 \mathrm{~nm}$ increase exponentially with laser power. However we find that the mean half life time for a trypanosome trapped at $15 \mathrm{~mW}$ laser power which is even strong enough to trap trypanosome duplets, is still longer than $30 \mathrm{~min}$. This allows for long time motility experiments, or antibody labelling experiments respectively. 


\section{CHAPTER SUMMARY AND CONCLUSIONS}

The combination of optical trapping techniques with high speed microscopy and microfluidic methods inherits a broad range of possibilities on trypanosome's studies. We were able to show that optical trapping of living trypanosomes is possible at reasonable low laser powers which allows for both high speed and long term examination of the cells. By calibration of the optical stall forces on trypanosomes, we could measure the propagation forces of cells with one and with two flagellae. We found that cell duplets generate precisely twice as much propagation force as a single cell. Moreover, we measured flagellar beating forces at the anterior end of a single cell to be bigger than $5 \mathrm{pN}$.

We estimated the refractive index of Trypanosoma cells and determined different favorable trapping positions within a trypanosome. These positions coincide with morphological features of the cells, particularly with the tubulin concentration. Consistently, the optical stall forces were determined to be highest at the region of the flagellar pocket. Furthermore the trapping positions are significantly different for tumbling cells in comparison to running cells. In order to estimate the loss of cell vitality, phototoxic effects in dependency on laser power have been quantified and range between $t_{\text {halflife }}=16 \mathrm{~min}$ and $t_{\text {halflife }}=73 \mathrm{~min}$ at minimum and maximum laser power respectively. Since none of the optical trapping experiments lasted longer than 6 min, we consider phototoxic effects to be negligible. 


\section{CHAPTER SUMMARY}

This chapter summarizes the principles of cellular motility in general, followed by a description of what is known about trypanosome motility so far and a precise analysis of trypanosome motility, which we observe in our experiments.

\section{PHYSICAL PRINCIPLES OF CELL MOTILITY}

Motility at the microscale is fundamentally different from our intuitive understanding of motility, which we know from the macroscale. A swimmer for example uses different acceleration rates when he is performing a fast backstroke with both arms and legs and then retreats them slowly to the front again. The inertial mass of the swimmer dominates over the friction forces in water. The mass of a bacterium on the other hand is so small that inertia becomes irrelevant and friction dominates the system. Such a condition can be experienced by the attempt of throwing a down feather through the air. Its mass is small compared to its surface, hence friction will immediately stop the feather after it has left the hand. The relation of inertial forces $F_{i}$ over viscous forces $F_{v}$ is given by the Reynolds number

$$
\operatorname{Re}=\frac{F_{i}}{F_{v}}=\frac{v \cdot l \cdot \rho}{\eta}
$$

where $v$ is the velocity, $l$ is a typical length scale, $\rho$ is the density and $\eta$ is the dynamic viscosity of the fluid. If the Reynolds number is very small $\mathrm{Re} \ll<1$, friction dominates and if it is high, inertia is the dominating force in the system ( $\underline{52})$.
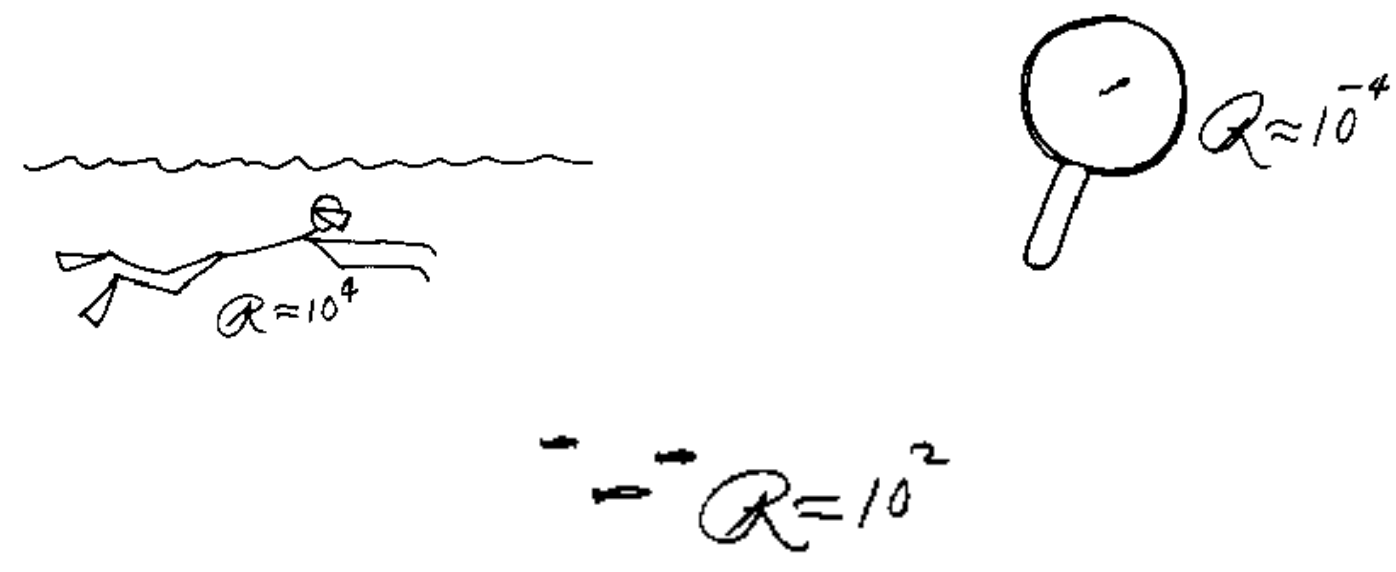

Fig. 4.1: Sketch of different Reynolds number $(R=\operatorname{Re})$ swimmers by E. M. Purcell

The Reynolds number of a trypanosome with $l=20 \mu \mathrm{m}$ which is swimming in water $\left(\rho=10^{3} \mathrm{~kg} \cdot \mathrm{m}^{-3}, \eta=0.001 \mathrm{~Pa} \cdot \mathrm{s}^{-1}\right)$ at a velocity of $v=20 \mu \mathrm{m} \cdot \mathrm{s}^{-1}$ is very small with $\mathrm{Re}=4 \cdot 10^{-5}$. 
To better understand the impact of low Reynolds numbers on the life of a trypanosome, it is helpful to calculate the distance it would drift if it suddenly stops swimming. To calculate the stopping distance, it is necessary to assume a friction of a trypanosome, given by the Stokes drag equation for a sphere.

$$
F_{\text {Stokes }}=6 \cdot \pi \cdot \eta \cdot r \cdot v=\beta \cdot v
$$

The dynamic viscosity of the medium is given for water with $\eta=1 \cdot 10^{-3} \mathrm{~Pa} \cdot \mathrm{s}$, where the radius for a trypanosome is similar to a sphere of $r=3 \cdot 10^{-6} \mathrm{~m}$ (chapter 3 ) which gives a friction coefficient of $\beta=5.6 \cdot 10^{-8} \mathrm{~kg} \cdot \mathrm{s}^{-1}$. To calculate the force needed to accelerate a trypanosome, friction is added to

$$
F=m \cdot a+\beta \cdot v=m \cdot \frac{d v}{d t}+\beta \cdot v
$$

where the mass of a trypanosome is given by $m=1.13 \cdot 10^{-13} \mathrm{~g}$ and the typical velocity is estimated by $v=20 \cdot 10^{-6} \mathrm{~m} \cdot \mathrm{s}^{-1}$. The motion given by the differential equation 4.3 can be described by an exponential decay of the velocity over time ( $\underline{52})$ with

$$
v(t)=\frac{F}{\beta}\left[1-\exp \left(-\frac{t}{\tau}\right)\right]
$$

hence the breaking time $\tau$ can already be calculated by

$$
\tau=\frac{m}{\beta}
$$

and is $\tau=2 \mu \mathrm{s}$, which is very short even on the microscale. The resulting breaking distance is given by

$$
x=\int_{0}^{\infty} v(t) d t=\int_{0}^{\infty} v(0) \exp \left(\frac{-t}{\tau}\right) d t=v(0) \tau
$$

and is as small as $x=4 \cdot 10^{-11} \mathrm{~m}$. This is a hundred times less than the thickness of a trypanosome cell membrane itself which is miniscule, even on the microscale.

The impact on life at such conditions is the evolution of completely different strategies of locomotion for microorganisms in comparison to multicellular organisms like fish, bird, or men respectively. 


\section{BIOLOGICAL PRINCIPLES OF CELL MOTILITY INTRODUCTION}

Active cellular motion is found in many variations throughout the kingdoms of life but those we are aware of are always based on only a few biophysical mechanisms (17) which can be simplified as:

\section{CRAWLING MOTION AND FLAGELLAR MOTION}

Crawling motion is the result of cellular reshaping, which is a capability of almost any living cell, but only to different extents. There are two different kinds of crawling motion, called amoeboid motion and pseudopodial motion which are both driven by dynamic changes in the structure of the cytoskeleton and cytosole.

\section{THE CYTOSKELETON}

The cytosol of cells is filled by a complex fibrous network, called cytoskeleton which determines the cell structure, but also facilitates its reshaping and therefore its motion. It is comprised of three main elements:

1. Stiff microtubules (diameter $D=24 \mathrm{~nm}$ ) capable of withstanding pushing forces and involved in chromosome segregation.

2. Actin microfilaments $(D=7-9 \mathrm{~nm})$ are the main building component of the cellular cortex. They represent the ropes that are needed to contract a cell or a muscle in effect.

3. Intermediate filaments represent the third group of fibrous proteins, where the name gives the size in range: the diameter is in between actin and microtubules. Prominent intermediate filaments are keratin, desmin and vimentin. They are as well involved in generation of forces and structural integrity of the cell.

Mechanical forces are generated by motor proteins associated with the cytoskeleton as well as the polymerisation of actin and microtubule fibres, driven by the hydrolysis of ATP and GTP (the "fuel of the cell"). While the polymerisation of filaments can drive the directed expansion of the cell, motor proteins can displace fibres alongside each other and bend e.g. a flagellum or carry cargo along a filament like in cytoplasmic flow.

Several types of motor proteins are known to interact specifically with certain filaments. Microtubules interact with kinesin or dynein in the case of the trypanosome flagellum and the myosin proteins work specifically on actin fibres. 


\section{AMOEBOID LOCOMOTION}

A well known example of amoeboid motion is given by the giant Amoeba proteus. It is a protozoan cell which can grow to a size of $l=1 \mathrm{~mm}$ and propagate with velocities up to $v=$ $3 \mathrm{~mm} \cdot \mathrm{min}^{-1}$. Amoeboid motion is driven by a process called hydraulic contraction, a cyclic process of cytosol polymerisation and depolymerisation: a constant stream of low viscous endoplasm towards the anterior cell pole is maintained by a combination of cytoplasmic transport and hydraulic contraction of the posterior end. Thereby the liquid endoplasm expands the anterior membrane and polymerizes into high viscous ectoplasm at the outer rim (Fig 4.2). The posterior ectoplasm depolymerises again and is pushed forward by myosin driven contraction of the posterior actin network. Carried forward, it again contributes in further membrane expansion and the propagation cycle is completed (53).

\section{Amoeboid locomotion}

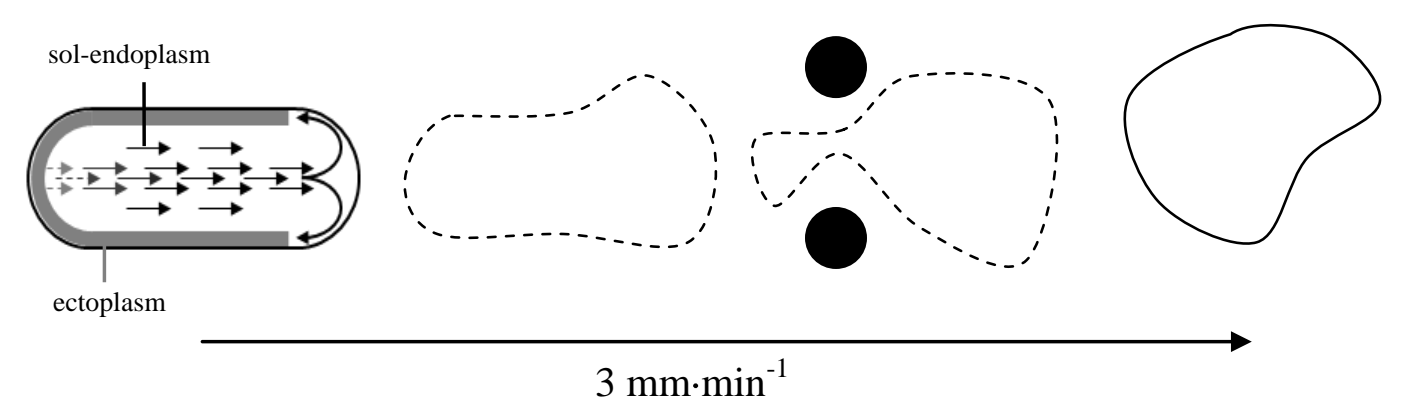

Fig. 4.2: Sketch of hydraulic contraction motion of Amoeba

Several examples exist for this kind of locomotion, but it is always partially accompanied by pseudopodial motion, like for example in neutrophiles or Dictyostelium, $(\underline{54}, \underline{55})$.

\section{PSEUDOPODIAL LOCOMOTION}

Fibroblasts are well known examples for motility, mainly driven by lamellipodial spread. The lamellipodium is a membraneous outgrowth of the cell, also called pseudopodium. It is driven by ATP dependent actin polymerisation and determines the new direction of motion. A focal contact to the substrate is established at the tip of the pseudopodium and so called actin stress fibres spread out to connect the new anterior end with the opposite side of the cell. 


\section{Pseudopodial locomotion}

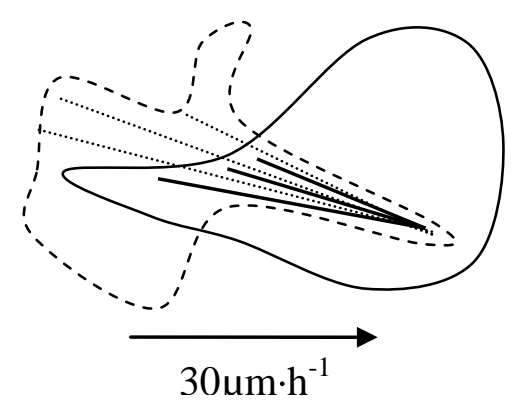

Fig. 4.3: Sketch of pseudopodial motion of a fibroblast.

The posterior part detaches from the old adhesion site and gets pulled towards the front by contraction of the stress fibres (Fig. 4.3, $\underline{56}$ ). Crawling motility is based on substrates like soil or tissue, but motility in fluid environments is mostly driven by flagellar motion.

\section{FLAGELLAR LOCOMOTION}

Flagellar locomotion marks the highest degree of specialisation on fast cellular motility. Eukaryotic flagella and cilia are highly conserved organelles, ubiquitously spread throughout the animal kingdom. Both are different words for the same basic structure, they only differ in their way of motion: a flagellum is beats, while a cilium waves (Fig. 4.4).

\section{Flagellar locomotion}
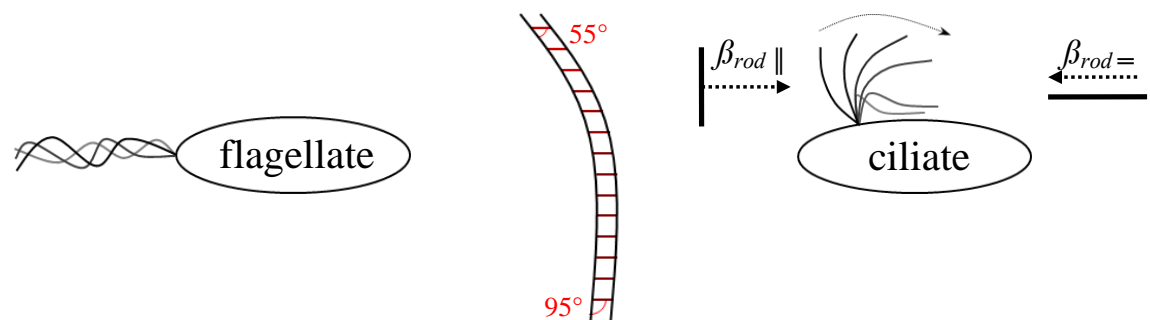

Fig. 4.4: Sketch of the flagellar beat, waving patterns and the microtubule bending mechanism

The basis of every flagellum or cilium is the microtubule axoneme, as described in chapter 1 . The bending motion which is propelling the cell, is induced by ATP driven dynein motor proteins. By conformational changes of these dynein motors, some of the outer microtubule doublets are displaced against each other, whilst they are still interconnected to the other doublets and hence have to give way in means of bending. Direction, velocity and amplitude are precisely actuated by the cells, however the underlying mechanisms are still not completely understood and subject to intensive research. 
At low Reynolds numbers, a symmetry breaking is necessary to produce a net force direction (52). This can be accomplished by a flagellum undergoing well controlled asymmetric strokes, or wave like patterns. For a ciliate this is given by an asymmetric forward stroke which inherits less friction $\left(\beta_{\text {rod }} \|>\beta_{\text {rod }}=\right.$, chapter 3 ) than the backward stroke. For trypanosomes however, the motility pattern as well as the underlying mechanisms of control are not yet completely understood.

\section{TRYPANOSOME MOTILITY IN LITERATURE}

Unlike other cells that bear freestanding flagella or cilia, the trypanosome flagellum is attached along the entire cell body (chapter 1, and Fig. 4.5 below). This unique structure of trypanosomes has major effects on the motility pattern of the cell. The flagellar beat force is transmitted to the cell body, forcing it to deform and move as a unit with the flagellum in consequence $(\underline{16}, \underline{57})$
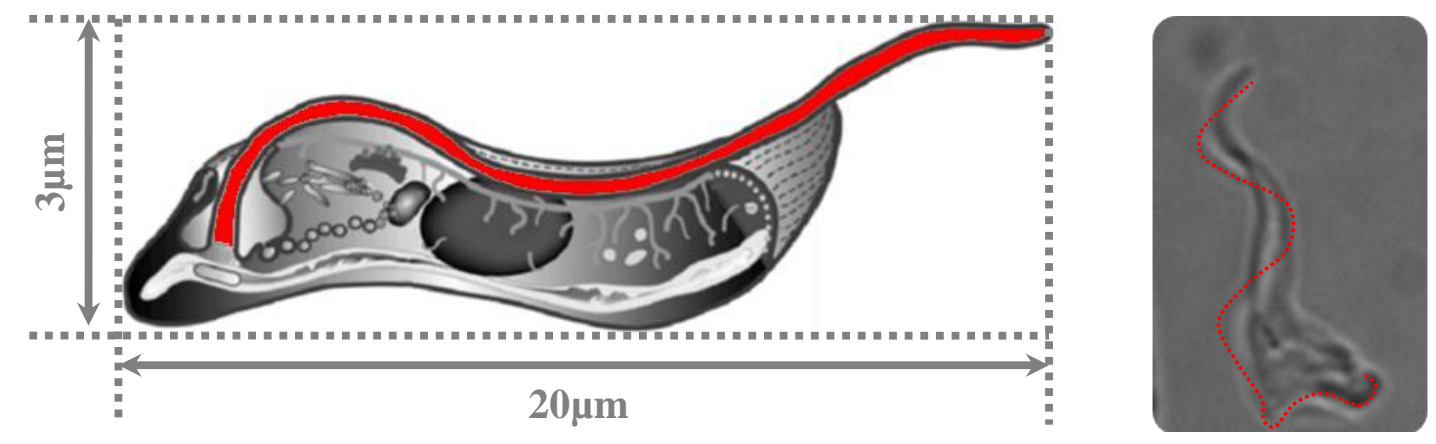

Fig.4.5: a) Sketch of Trypanosoma brucei brucei (Grünfelder et al. 2003). b) Brightfield image of $T$. $b$. brucei magnified 2000 times. The flagellum is indicated in red.

The unusual motility gave name to the genus Trypanosoma which can be translated as "drilling body". This descriptive term has then been referred to for a long time whenever trypanosome motility was mentioned $(\underline{58}, \underline{59})$. But the actual motility of trypanosomes is far more complex and less regular than just a simple helical motion with a certain direction.

When we first looked at trypanosomes we immediately saw that most of the cells do not make any progress, although moving rapidly in some sort of complex pattern. Those cells that did propagate however, revealed a motion that looks similar to a helical path, but is accompanied by rapid deformations in cell shape and changes in direction.

Recent findings by Sravanti Uppaluri et al. (in review PLoS, 2010) and Rodriguez et al. (드, 2009) represent first approaches to describe the complex motility of trypanosomes to its full 
extent. Uppaluri et al. showed that the different motility patterns observed for running and tumbling cells in a BSF Trypanosoma brucei brucei population, are due to differences in cell body stiffness. While stiff cells show a mean persistence length in directional motility of $L_{p}=207 \mu \mathrm{m}$, tumbling cells do not show any directional motility at all as a result of significantly reduced cellular rigidity.

Rodriguez et al. compare procyclic trypanosomes with blood stream forms by high resolution differential interference contrast microscopy. The results show that both kinds of cells propagate through bihelical waves of different chirality, contrary to the common corkscrew like description that was previously assumed in literature. The described bihelical waves induce a kink in cell morphology, where the posterior part of the cell undergoes a rocking motion at about $5 \mathrm{~Hz}$, while the anterior part swings counter clock and clockwise at a frequency of $19 \mathrm{~Hz}$. However, the limitations of field of view in common microscopy do not allow for high quantity and long term measurements on trypanosome motility.

Using optical trapping methods in combination with automated image processing procedures, we were able to overcome the limitations in quantity and observation time on trypanosome motility. The results are given in the next chapter and compared with findings by Uppaluri S..

\section{Rotating trapanosome}

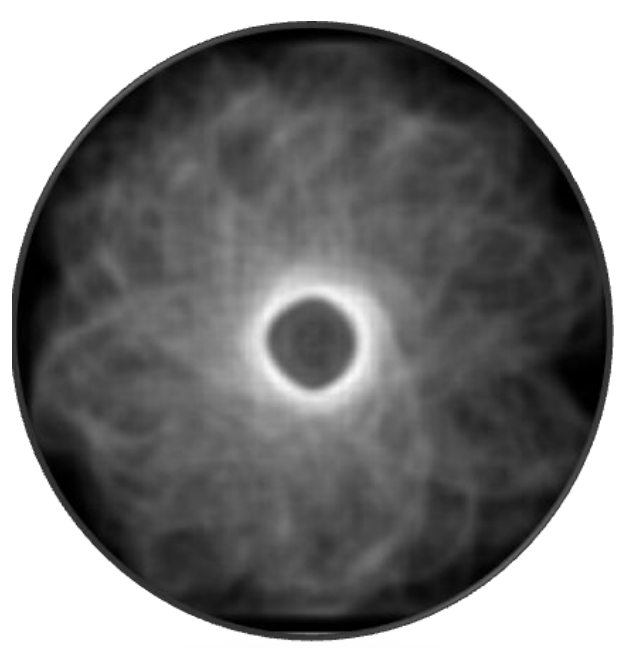

Fig. 4.6: Image overlay of a rotating BSF trypanosome within optical confinement 


\section{TRYPANOSOME MOTILITY IN CONFINEMENT}

\section{INTRODUCTION}

As parasites, trypanosomes have to cope with ever changing environmental conditions of both chemical as well as physical nature. Changing from endothermal host to ectothermal vector, they have to adapt to new temperature conditions and change their metabolism from glucose uptake to amino acid nutrition (60). During both their life and cell division cycle, they also have to migrate through various kinds of tissues into the lymphatic system, the blood stream and into tissue again. All these environments are very different in their spatial structure and trypanosome motility has to be well adapted to these conditions.

Using PDMS microfluidic devices, we constructed microchannels of varying sizes in order to examine $B S F$ trypanosome motility within confinements that mimic dimensions of both the blood capillary and the interstitial (spaces between cells and lymph) habitat.

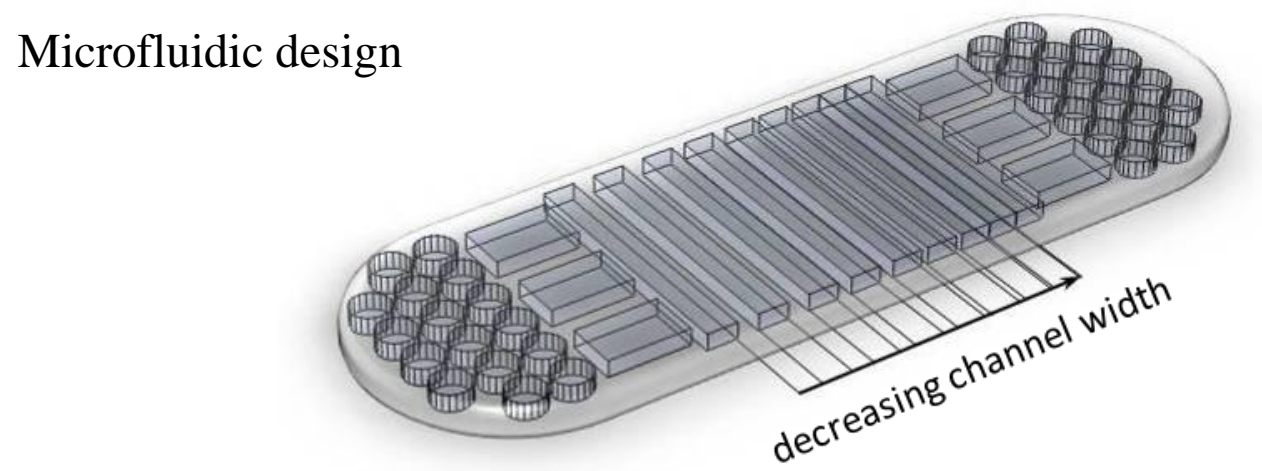

Fig. 4.7: Sketch of the PDMS microfluidic stop flow device used in the experiment. Channel sizes vary from 2 to $20 \mu \mathrm{m}$ in width over a constant height of $7 \mu \mathrm{m}$.

The first part of the experiment describes the cell orientation and ability to change direction within small confinements. The impact of confinement on cell velocities and propagation is discussed in the second part.

\section{EXPERIMENT}

The experiment was conducted in PDMS microfluidic stop flow devices with a constant height of $7 \mu \mathrm{m}( \pm 1 \mu \mathrm{m})$ and varying channel width, ranging from 1 to $20 \mu \mathrm{m}( \pm 0.5 \mu \mathrm{m})$. Observations were done in brightfield illumination using an Olympus BX 61 microscope. The 20, 10 and $5 \mu \mathrm{m}$ channels were observed using an Olympus LMPlanFl 50x/0.5 $\infty$ objective, whereas the $2 \mu \mathrm{m}$ channel was observed using an Olympus UPlanFl 60/1.25 $\infty$ oil immersion objective. 
The emission pathway of the microscope was modified using an extra field stop aperture of $D=10 \mathrm{~mm}$ to generate homogeneous illumination on the camera CCD sensor. Each cell was recorded for $t=100 \mathrm{~s}$ at a framerate of $100 \mathrm{fps}$ using a Phantom Miro 3 camera. The focal plane was slightly defocused to gain contrast of cell shape and increase signal to noise ratio in automated image processing.

\section{Preparation of Cells}

Trypanosoma brucei brucei and were prepared as described in chapter 2.4. The time of measurement was restricted to $30 \mathrm{~min}$ for all cells. After successful measurements the cells were killed using a $405 \mathrm{~nm}$ laser focussed on the center of the focal plane to prevent from repeated measurements of the same cell.

\section{RESULTS AND DISCUSSION}

\section{MICROFLUIDIC STOP FLOW}

Trypanosome propagation velocities of tumbling cells approach values $v_{n e t}<1 \mu \mathrm{m} \cdot \mathrm{s}^{-1}$ (S. Uppaluri et al. 2010, in review). In order to analyze trypanosome motility in confinement, it is necessary to create very stringent stop flow conditions, with disturbances smaller than $\Delta v_{\text {fluid }}<0.5 \mu \mathrm{m} \cdot \mathrm{s}^{-1}$. We developed a microfluidic stop flow device, with channels orientated perpendicular to the pressure gradient, existing between inlet and outlet (Fig. 4.7). No significant disturbances other than Brownian motion are detected even over long timescales $t_{\text {observe }}>100 \mathrm{~s}$ (Fig. 4.8).

\section{Microfluidic stop flow device}
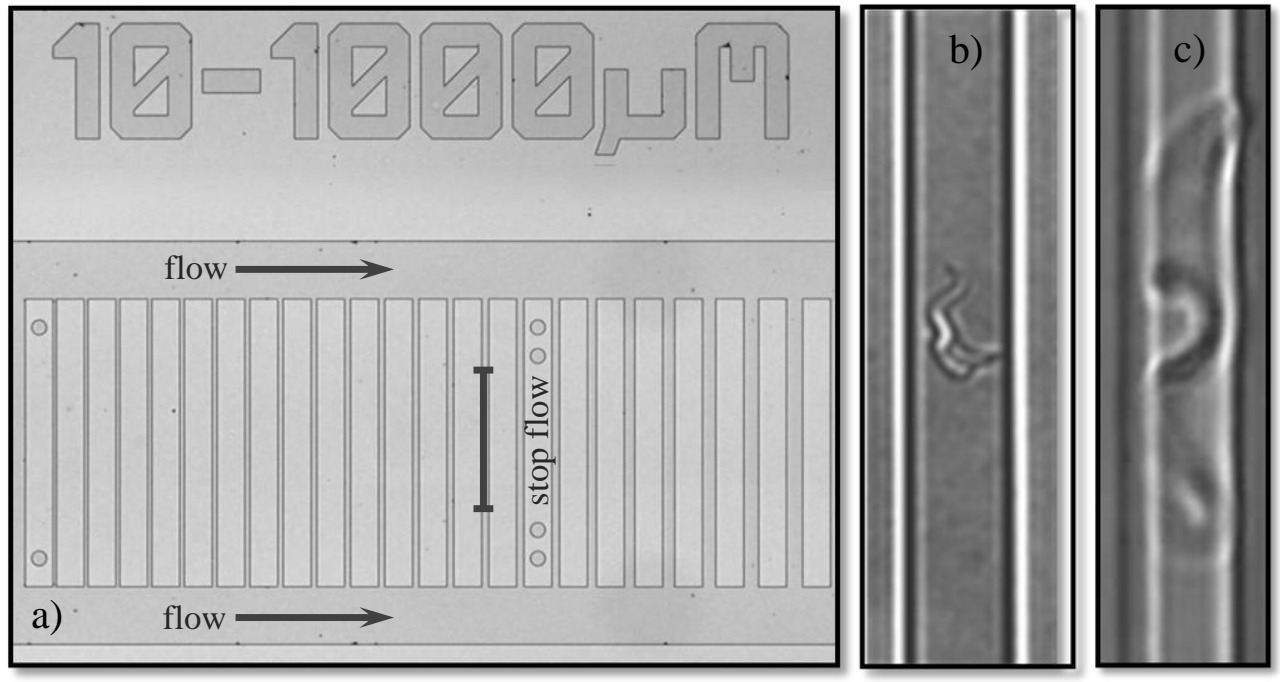

Fig. 4.8: a) Image of a microfluidic stop flow device used in experiment $(m=20 \mathrm{x})$. b) Trypanosome in a 10 $\mu \mathrm{m}$ microfluidic stop flow channel $(m=980 \mathrm{x})$. c) Trypanosome in a $2 \mu \mathrm{m}$ stop flow channel $(\mathrm{m}=2500 \mathrm{x})$. 
Flow velocity within an $A=10 \mu \mathrm{m} \times 7 \mu \mathrm{m}$ (cross section) stop flow channel has been determined by the particle trace of a $D=3 \mu \mathrm{m}$ polystyrene sphere, shown in figure 4.9.
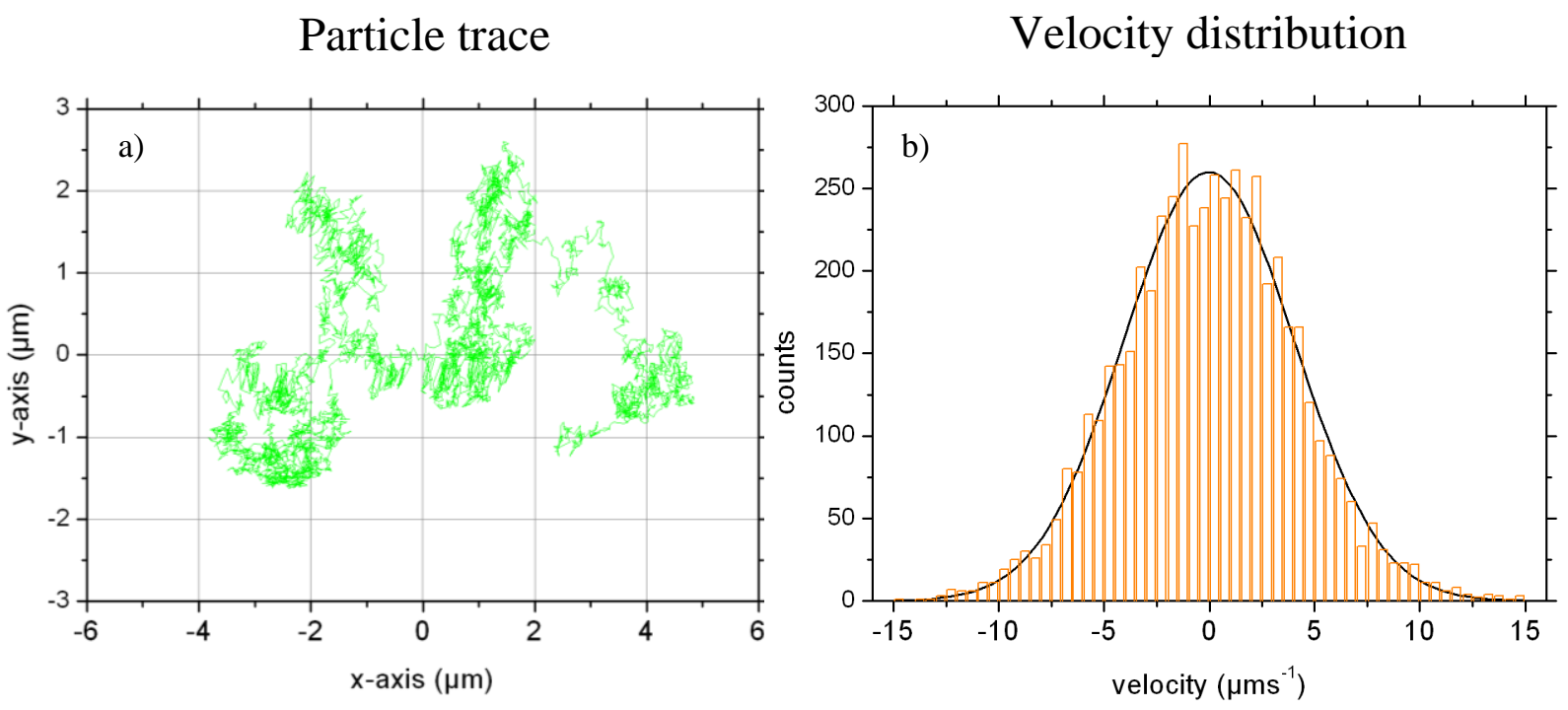

Fig. 4.9: a) Particle trace of a $D=3 \mu \mathrm{m}$ polystyrene sphere in an $A=10 \mu \mathrm{m} \times 7 \mu \mathrm{m}$ microfluidic channel b) Particle velocity distribution. Orange bars: X-axis velocity distribution counts; black line: Gaussian fit (center $=0.02 \mu \mathrm{m} / \mathrm{s} ; F W H M=9.54 \mu \mathrm{m} / \mathrm{s}$ ) in stop flow.

The particle trace was recorded at $53.15 \mathrm{fps}$ over $100 \mathrm{~s}$, as it is the duration time for the experiments on trypanosome motility in confinements. The mean velocity in $x$-axis direction is determined by a Gaussian fit to the velocity distribution. The stop flow condition $\Delta v_{\text {fluid }}<$ $0.5 \mu \mathrm{m} \cdot \mathrm{s}^{-1}$ is matched well by the experimental condition of $\Delta v_{\text {fluid net }}=0.02 \mu \mathrm{m} \cdot \mathrm{s}^{-1}$. Stop flow is maintained even with flow in the perpendicular supply channels (Fig. 4.8). Therefore, this device can also be used to generate a stable, or even dynamic, concentration gradient along the channel direction, without any flow to examine chemotaxis of trypanosomes in future experiments to come. 


\section{ORIENTATION IN CONFINEMENT}

Trypanosome motility was analyzed in channels of four different widths $w: 2,5,10$ and 20 $\mu \mathrm{m}$. Each cell out of $n=20$ per channel was recorded over a time span of $t_{\text {observe }}=100 \mathrm{~s}$. Time resolution was $10 \mathrm{fps}$ for $w=2 \mu \mathrm{m}$ channels, 25 fps for $w=5 \mu \mathrm{m}$ channels and $100 \mathrm{fps}$ for both $w=10 \mu \mathrm{m}$ and $w=20 \mu \mathrm{m}$ channels. Trypanosome imaging has been fully automated using image processing techniques as described in chapter 2 . The cell orientation in relation to the channel width has been analyzed by the angle of displacement $\alpha$ as well as by the orientation of the major cell axis $\beta$ as depicted in figure 4.10 .

\section{Direction of motion and cell orientation}
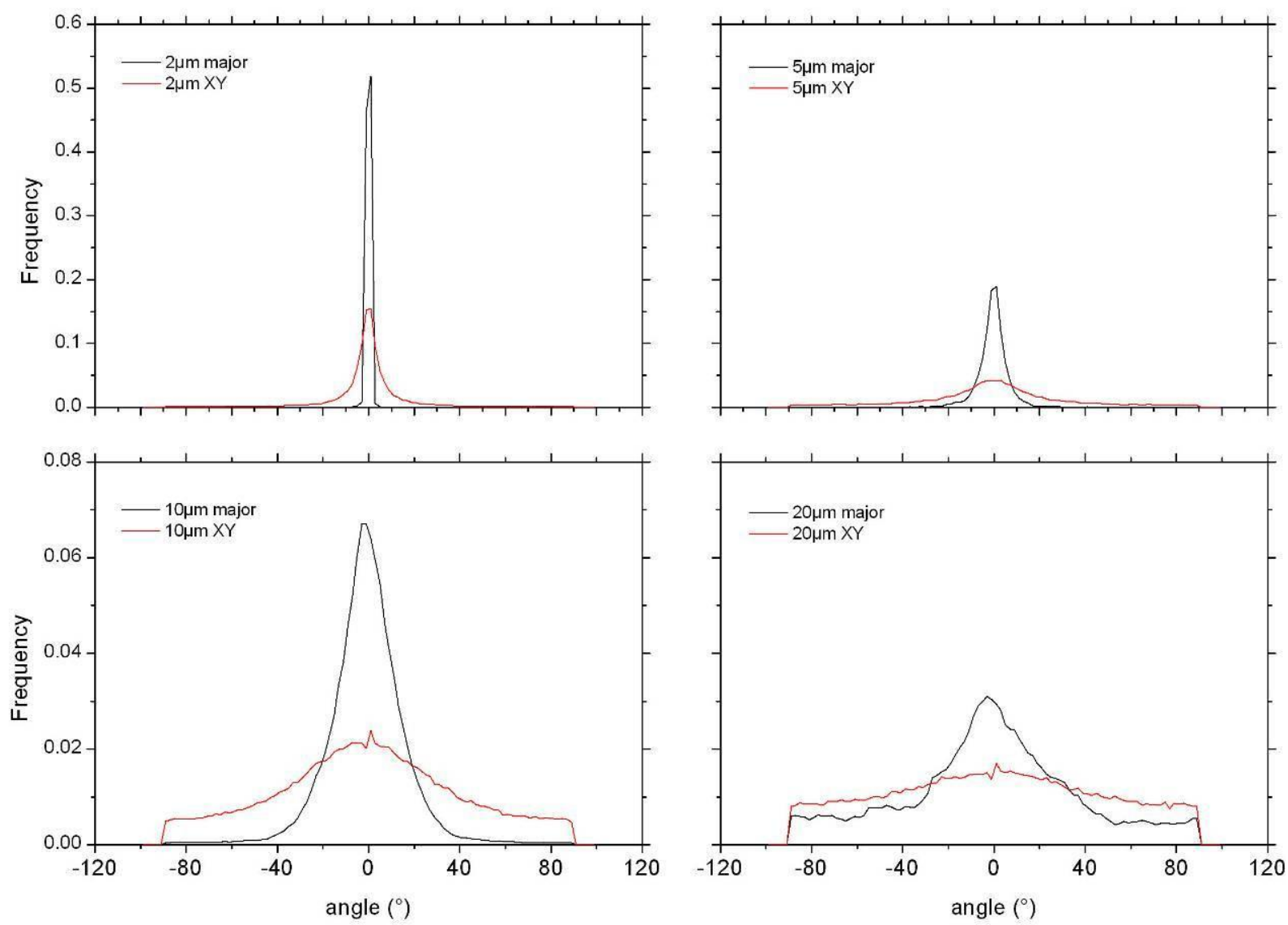

Fig. 4.10: Cell axis orientation distribution and angle of displacement distribution over channel width. Black lines: major cell axis orientation; red lines: angle of displacement.

The direction of motion is given by the angle of displacement $\alpha$ (Fig. 4.10, red lines). It is already limited in channels that are as wide as the cells are long with $w_{\text {channel }}=l_{\text {cell }}$. The angular spread of motion direction, fitted by a Gaussian function, is $70^{\circ}$ (FWHM, Fig. 4.10) instead of $180^{\circ}$ for a free cell undergoing non directional motion ('tumbler').

Nevertheless, almost all angles of motion are realized, hence cell motion is only weakly restricted in channels of $20 \mu \mathrm{m}$ width. 
Cell orientation $\beta$ (Fig. 4.10, black lines), on the other hand, already shows stronger limitations with an angular spread of only $44^{\circ}$ (FWHM, Fig. 4.10). The difference between angular displacement and cell orientation limitation in confinement already shows that trypanosome movement is not limited in forward direction, but can also undergo sideways motions. The development of cell orientation and direction of displacement is shown in figure 4.11 .

\section{Direction of motion and cell orientation}

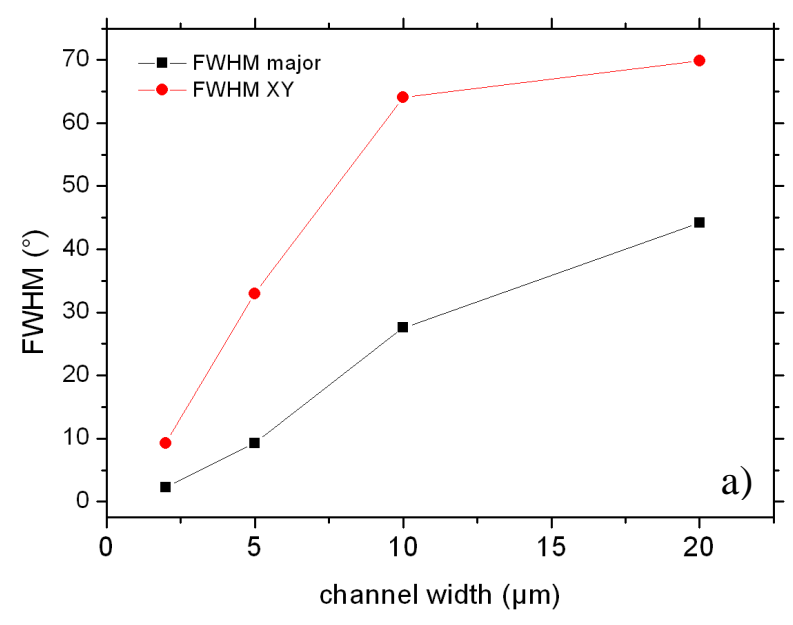

b)

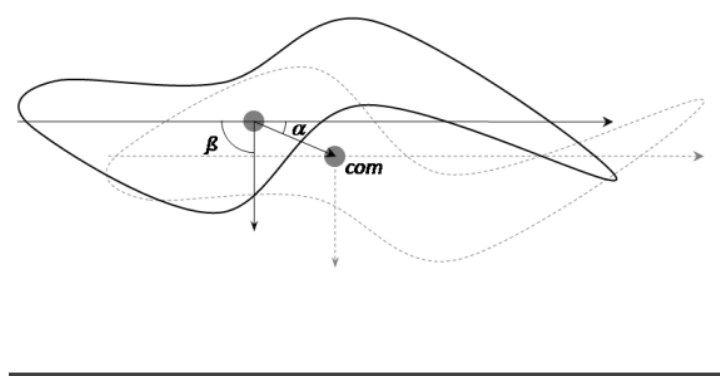

Fig. 4.11: a) Cell axis orientation distribution and angle of displacement distribution over channel width. Black lines: major cell axis orientation; red lines: angle of displacement. b) Illustration of the measurements. $\beta$ : major cell axis orientation; $\alpha$ : angle of displacement.

As expected, the major cell axis (cell orientation) aligns parallel to the channel walls with decreasing width, but while cell alignment almost increases linearly, the angle of displacement decreases strongly only at lateral confinements $w \leq 10 \mu \mathrm{m}$. An explanation of a stronger alignment of the major cell axis in comparison to the angle of displacement is sketched in figure $4.11 \mathrm{~b}$. Whilst geometric constraints have a strong impact on the orientation of the major axis, for channel widths $w>10 \mu \mathrm{m}$, the com angle displacement does not experience a strong influence of the confinement. At $w \leq 10 \mu \mathrm{m}$ the amount of wall contacts increases, as does the friction between the trypanosomes and the walls. Due to the increased friction, the com angle displacement, perpendicular to the channel, is dramatically reduced and the freedom of angle displacement is strongly restricted. 


\section{Propagation In CONFINEMENT}

Two different velocities were compared in the following experiment. The over all velocity $v_{\text {absolute }}$ is given by the center of mass displacement in both $x$ - and $y$-axis direction and the $x$ directional velocity $v_{x}$ is given by the $x$-axis displacement.

\section{Velocitiy distributions in confinements}
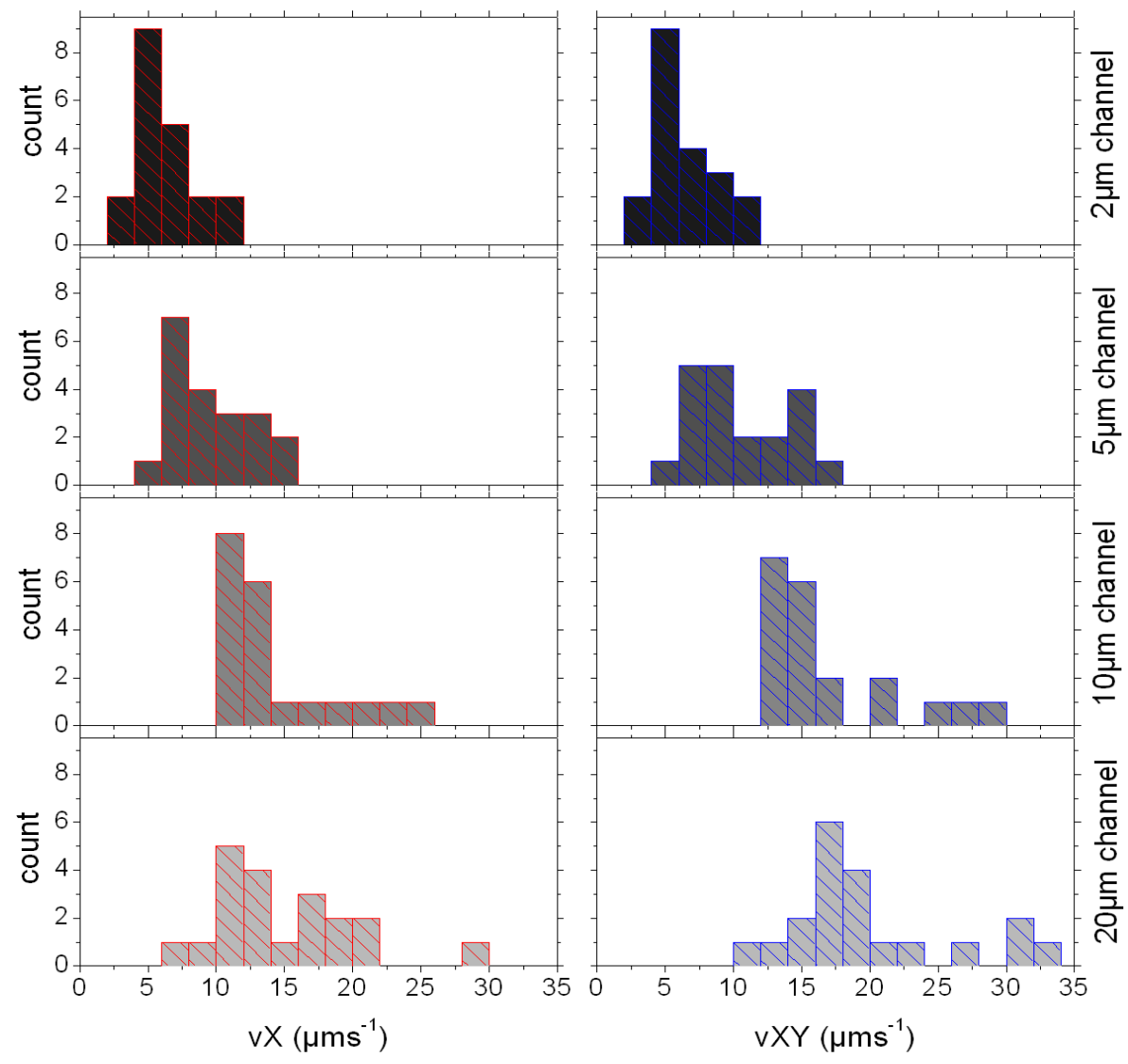

mean velocities

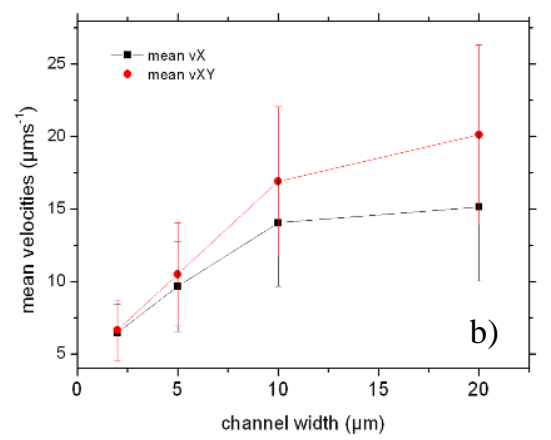

ratio

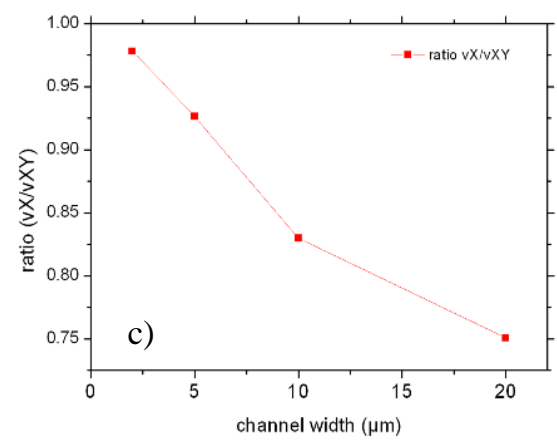

Fig. 4.12: a) Mean velocity distributions over channel width. Grey red bars: mean $v_{x}$ distribution; grey blue bars: mean $v_{\text {absolute }}$ distribution. b) Mean velocities. Red line: $v_{x}$; black line: $v_{\text {absolute }}$. c) Ratio $v_{x} / v_{\text {absolute }}$.

In $w=20 \mu \mathrm{m}$ channels $v_{\text {absolute }}=20 \mu \mathrm{m} \cdot \mathrm{s}^{-1}$ is higher than $v_{x}=15 \mu \mathrm{m} \cdot \mathrm{s}^{-1}$, indicating that there is almost no confinement present in cell motility (where $v_{x} / v_{\text {absolute }}=1 / \sqrt{ } 2=0.707$ for free motility $v_{x}=v_{y}$ ). 
With decreasing channel width, the geometric constraints increase and the ratio of $v_{x} / v_{\text {absolute }}$ approaches 1 (almost no $v_{y}$-component) at a mean velocity of $v_{\min }=6.5 \mu \mathrm{m} \cdot \mathrm{s}^{-1}$. Although the $x$-axis displacement velocity decreases with channel width (Fig. 4.12), the net travel velocity in $x$-direction, calculated by the max net travel distance and detected for a time span $\geq 10 \mathrm{~s}$, changes in different ways.

\section{Travel velocities}

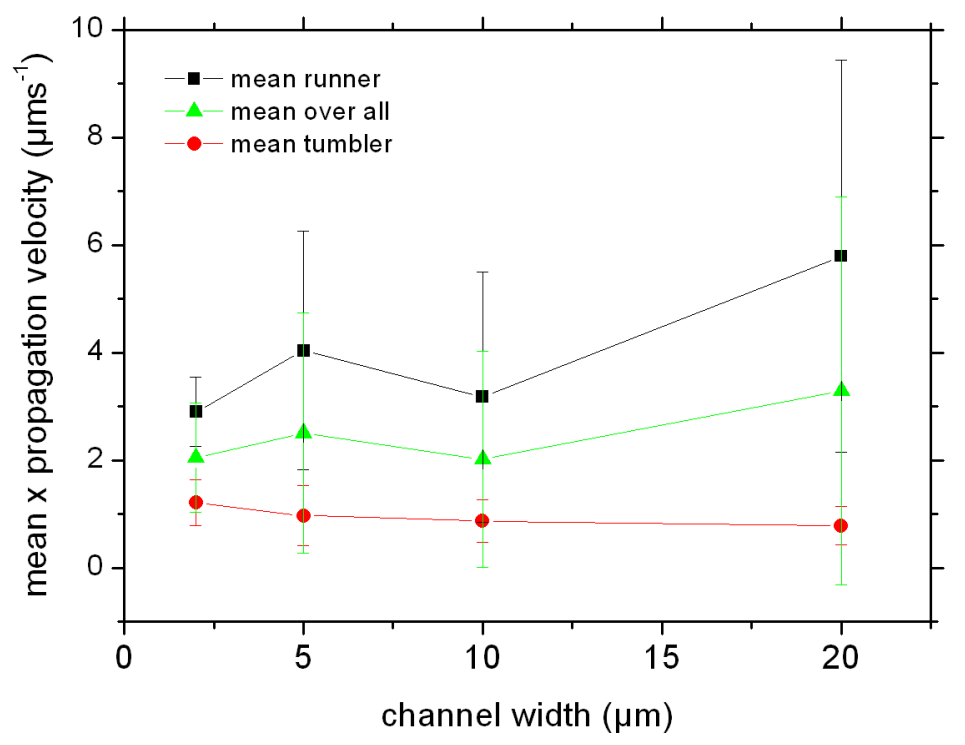

Fig. 4.13: a) Net travel velocities for running and tumbling cells. Green line: mean $v_{\text {travel }}$ over all; black line: mean $v_{\text {travel }}$ runners; red line: mean $v_{\text {travel }}$ : tumblers; black, green, red bars: error bars as STD of data.

Running cells with strong persistent motion travel fastest in the least confining $w=20 \mu \mathrm{m}$ channels, $v_{\text {travel,runner }}=5.2 \mu \mathrm{m} \cdot \mathrm{s}^{-1}$ and slow down by almost $50 \%$ in $w=2 \mu \mathrm{m}$ channel confinements. In comparison, tumbling cells travel with $v_{\text {travel,tumbler }}=0.8 \mu \mathrm{m} \cdot \mathrm{s}^{-1}$ in the widest channels and they are actually forced into an accelerated travel of about $35 \%$ to a velocity of $v_{\text {accelerated }}=1.2 \mu \mathrm{m} \cdot \mathrm{s}^{-1}$. Whereas directional persistent cells are decelerated by the increased friction with the walls in smaller channels, a different behaviour is observed for tumbling cells: confinement induces a weak, but yet directional motion (Fig 4.13). 


\section{TRYPANOSOME MOTILITY IN OPTICAL CONFINEMENT INTRODUCTION}

Trypanosome motility is very complex and changes, not only with morphology during a life cycle, but also within a population of trypomastigotes. Optical trapping of trypanosomes provides two basic methodological advantages for motility analysis:

1. High resolution long term imaging of rapid moving organisms. As a matter of optics, high resolution microscopy is accompanied with a small field of view limiting the observation time for rapidly propagating cells. Automated cell tracking using fast translation stages in combination with real time image processing is a possibility to follow even fast moving cells. Using either water or oil immersion objectives in combination with step wise stage translation however, induces non negligible flow disturbances in the fluid, even through the coverslip glass. We faced flow disturbances up to $v_{\text {induced }} \leq 50 \mu \mathrm{m} \cdot \mathrm{s}^{-1}$ induced by sample translation, although using low viscosity immersion oil. As a result, cell motility itself will be disturbed and image processing becomes more complicated due to the factor of variable stage velocity correction.

2. Optical trapping provides a view along the axis of revelation for cells which naturally undergo super helical motion. Such motion is not hindered within optical confinement, but translated into a high amplitude rotational motion. In a $2 \mathrm{~d}$ confinement, needed to limit the cell to the focal plane in conventional microscopy, such a motion is disturbed by wall contacts and the projected amplitudes of rotation are smaller and hence detected with less accuracy.

Description and analysis of trypanosome motility within optical confinement and evolution of trypanosome motility over time are the subjects of the following chapter.

\section{EXPERIMENT}

The experiment is described in chapter 3.2, the presented data are derived on basis of the running and the tumbling cells, described in chapter 3.4, to allow for direct comparison with earlier results. 


\section{RESULTS AND DISCUSSION}

\section{ROTATIONAL MOTILITY}

All cells which move in a regular rotational pattern rotate in the same direction which is clockwise if seen from the posterior to the anterior pole. As shown in figure 4.14, the cells can orient either towards the light source, or pointing away from it, detectable by the diffraction pattern of the transmitted light (Fig. 4.14)

\section{Trypanosome rotation}
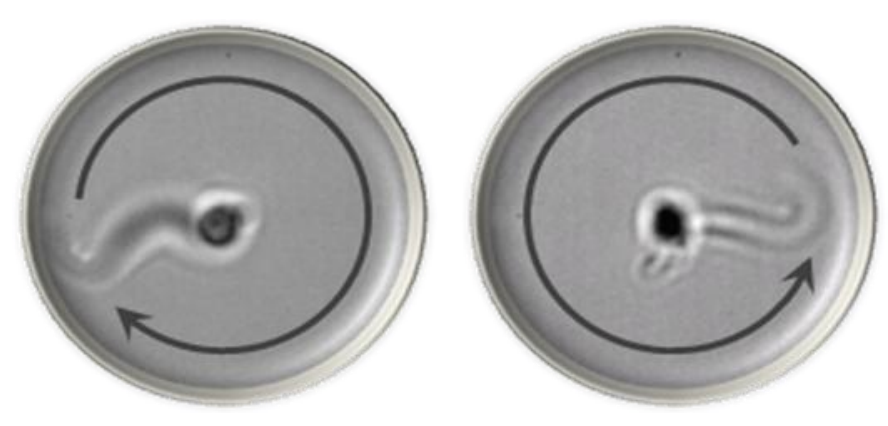

Fig. 4.14: Trypanosomes rotating in optical confinement. a) Anterior pointing towards light source: clockwise. b) Posterior pointing towards laser source: counter clockwise.

Cells which are capable of rotating within the optical trap may undergo a complex, possible superhelical motion in 3 dimensional free space. We understand persistent rotation as the equivalent to directional motion typical for running cells. Trypanosomes rotating over periods longer than $12 \mathrm{~s}$, will therefore be denoted as persistent walkers, or running cells, as defined by Sravanti Uppaluri et al.. Furthermore, we describe the changes in motility in high spatiotemporal resolution beyond time scales of only several seconds. 


\section{TRYPANOSOME FREQUENCIES}

In order to analyze the temporal structure of trypanosome motility, the autocorrelation of $\mathrm{x}$ axis center of mass displacement was analyzed. The correlation coefficient is given by

$$
y(m)=\sum_{n=0}^{M-1} f(n) g(n-m)
$$

with $f(n)$ and $g(n)$ as two signals of the same length, $M$. The magnitude of the correlation thereby shows the degree of similarity between the two signals, where negative values indicate anti-correlation and positive values a correlation (ㅁiginLabs).

Long term motility analysis of trypanosomes in optical confinement reveals many different overlaying frequencies within the autocorrelation spectrum of center of mass displacement. To determine the underlying structure, the time span of correlation has been successively reduced (Fig. 4.15). The lower observation limit $\left(10 \times f_{\min }\right)$ in time is given by the lowest rotation frequencies, observed to be approximately $0.45 \mathrm{~Hz}$, as recognized in figure 4.15 and 4.16 .

\section{Trypanosome time frame}

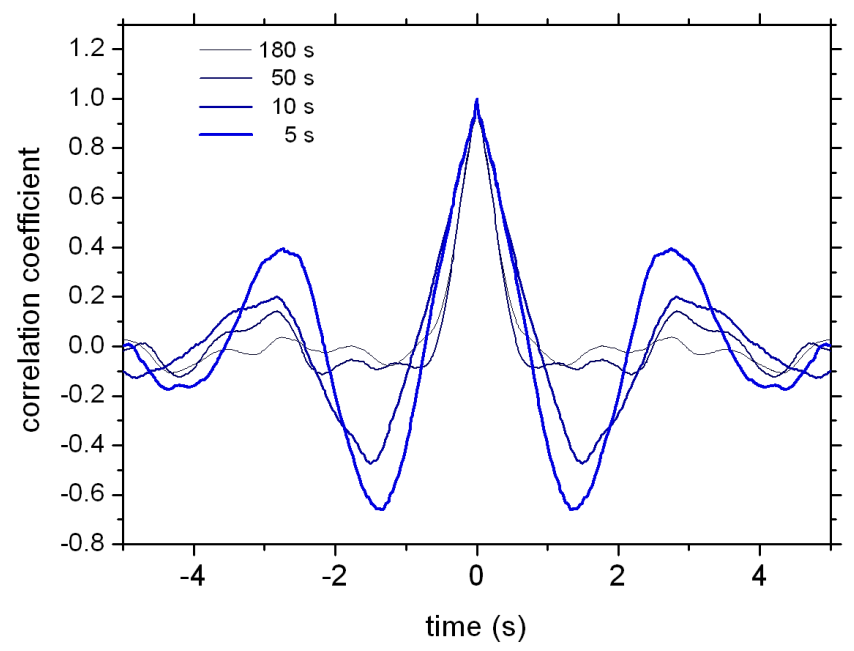

Fig. 4.15: Autocorrelation of $\mathrm{x}$-axis center of mass displacement of a single cell at a time interval of $0.01 \mathrm{~s}$. Blue lines: Autocorrelations over different time spans from 180 to $5 \mathrm{~s}$.

The strongest and most distinct correlation signal arises at the minimum timeframe of $5 \mathrm{~s}$. Therefore we assume that within a window of $5 \mathrm{~s}$, trypanosome motility does not change very much and that the predominating frequencies give rise to a strong signal in the autocorrelation function. The distance of the second peak to the center peak is $\Delta t=2.3 \mathrm{~s}$ equal to a frequency 
of $0.45 \mathrm{~Hz}$. We believe this frequency to represent the overall rotating frequency of a running trypanosome as shown in figure 4.16.

Zooming into the data, a second prominent frequency appears at $14.3 \mathrm{~Hz}$ atop the curve.

\section{Trypanosome motility patterns}

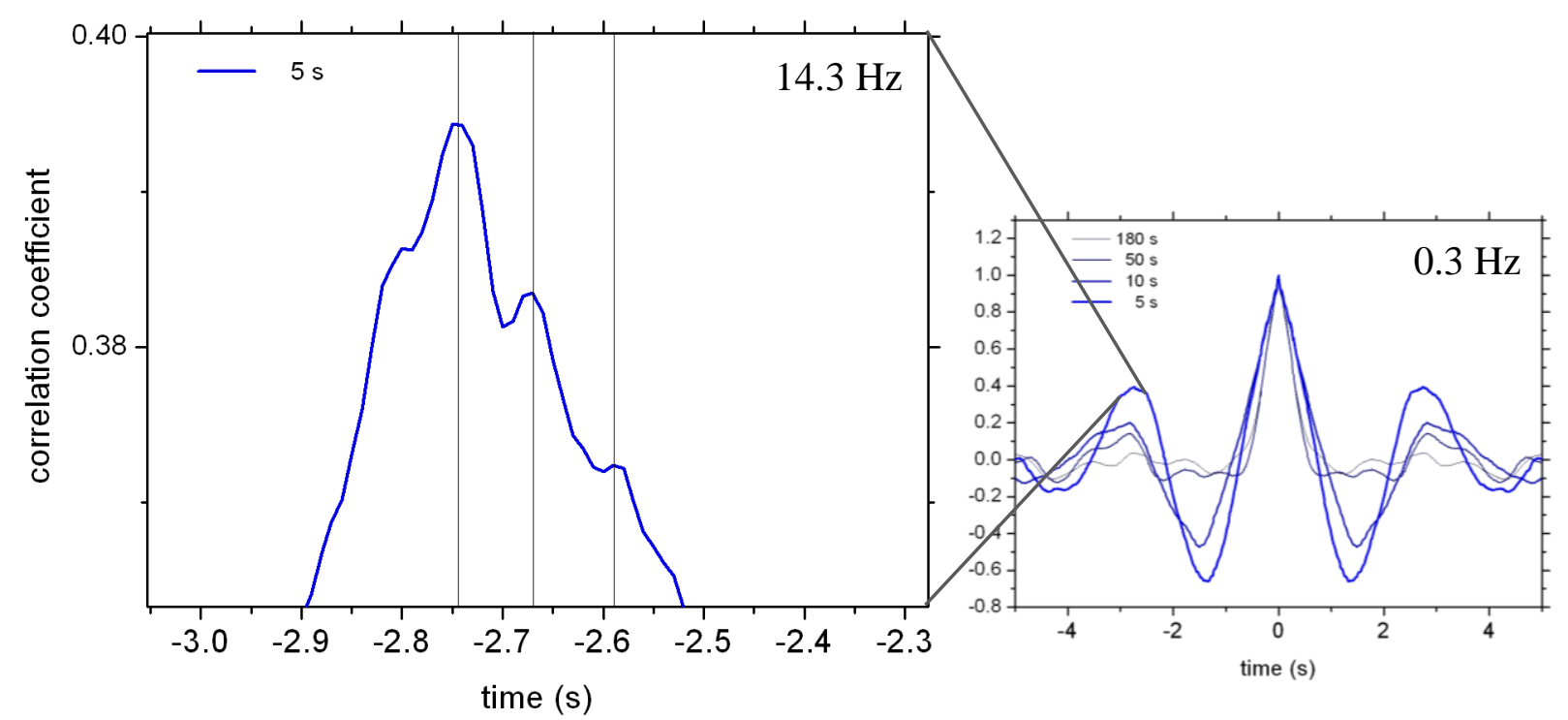

Fig. 4.16: Zoom into the second peak of Fig. 4.15. Blue line: autocorrelation function; gray lines: peak

To determine the origin of this second frequency, we analyzed the trace of the cell poles obtained from the same dataset with $t O_{\text {trace }}=t 0_{a c}$.

\section{Trypanosome motility features}
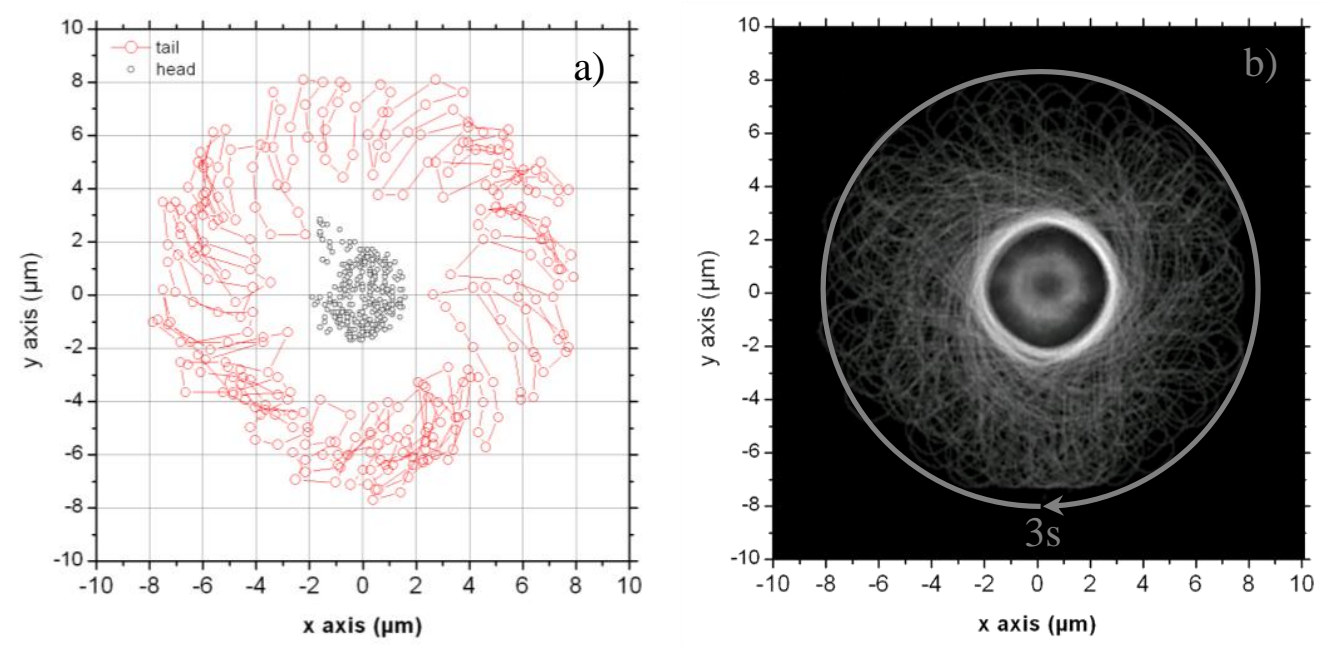

Fig. 4.17: a) Trace of anterior and posterior end of a trypanosome in optical confinement. The autocorrelation of figure 4.15 is based on the center of mass displacement of the same cell at the same time as given in this figure but for a period of $t_{a c}=5 \mathrm{~s}$ instead of $t_{\text {rotation }}=3 \mathrm{~s}$. Red line: anterior pole trace; gray line: posterior pole trace. b) 300 frames image overlay. 
The cell pole trace gives a rotation time of $2.7 \mathrm{~s}$, compared to $2.3 \mathrm{~s}$, obtained by the autocorrelation function. The remaining difference is due to deviations in cell motility which take effect on the bigger time frame of the autocorrelation $\left(t_{a c}=5 \mathrm{~s} ; t_{\text {trace }}=2.7 \mathrm{~s}\right)$.

Further more, the trace of the anterior tip reveals a most interesting pattern: whilst the cell is rotating around the center of the trap in 2.7 seconds, the anterior tip is undergoing a motion, best describes as reaching out and pulling in, at a frequency of $f_{\text {beat }}=14.8 \mathrm{~Hz}$. This motion is also seen by the center of mass displacement and given by its autocorrelation, as we see it in figure 4.16 with $f_{a c}=14.3 \mathrm{~Hz} \approx f_{\text {beat }}$.

This comparison clearly shows that the autocorrelation function of the center of mass displacement describes trypanosome motility frequencies, determined by high speed brightfield microscopy in full detail. Reducing motility to the center of mass displacement in turn, allows for high quantity analysis of cell motility by using automated image processing methods, as we have employed them for our experiments (chapter 2.3).

Analysis of the anterior tip motion revealed a mean amplitude of approximately $4 \mu \mathrm{m}( \pm 0.5$ $\mu \mathrm{m})$ and a frequency of 40 beats per rotation $f_{\text {beat }}=14.8 \mathrm{~Hz}$. The maximum tip velocity was determined with $v_{\max }=796 \mu \mathrm{m} \cdot \mathrm{s}^{-1}$, whilst the mean velocity was measured by the slope of the sum of tip displacement over time with $v_{\text {mean }}=277 \mu \mathrm{m} \cdot \mathrm{s}^{-1}$ (Fig. 4.18).

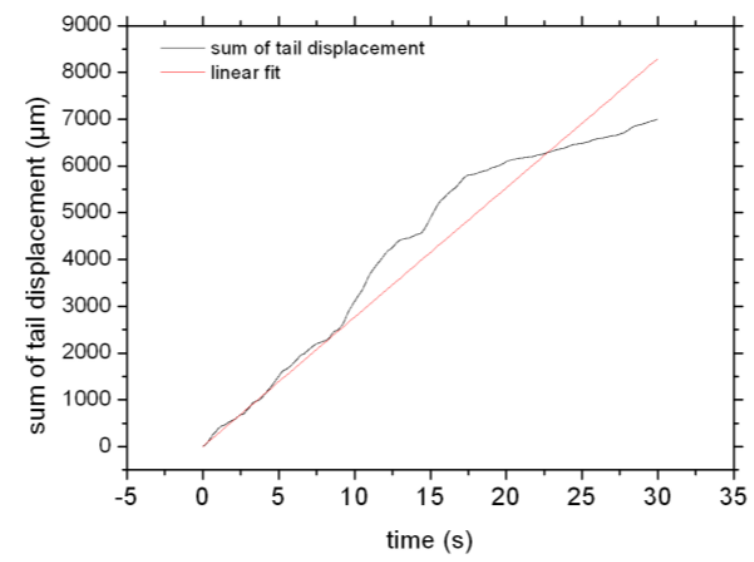

\section{Anterior tip velocity}

Fig. 4.18: Slope of sum of $x y$ displacement of the anterior tip over time. Black line: sum of xy displacement; red line: linear fit (slope: $277 \mu \mathrm{m} \cdot \mathrm{s}^{-1}$;

These findings are in very good agreement with the description of anterior swinging motions at a frequency of $19 \mathrm{~Hz}$, described by Rodriguez et al. The maximum tip velocity for $B S F$ trypanosomes is reported to be $v_{\max }=673 \mu \mathrm{m} \cdot \mathrm{s}^{-1}$, whereas the mean velocity is given as $v_{\text {mean }}=510 \mu \mathrm{m} \cdot \mathrm{s}^{-1}$. We consider the reduced mean velocity to be an effect of the optical confinement. Running cell motility is translated into a rotational motion when trapped at the flagellar pocket and the axial velocity component is completely compensated. Therefore, the flagellum will experience an increase in friction for every force contribution in axial direction, resulting in an overall decrease of the anterior tip velocity as we see in this measurement. The 
difference in frequency and maximum velocity, compared to the short time measurements of Rodriguez et al. however, is due to variations in trypanosome cell motility itself which will be described in detail in the following chapter.

\section{TEMPORAL DYNAMICS OF TRYPANOSOME MOTILITY}

The prior analysis revealed very detailed aspects of trypanosome motility on the short term. To further analyze the temporal dynamics of trypanosome motility, a moving frame autocorrelation comparison has been conducted. The over all observation time is $180 \mathrm{~s}$, with a step size of $10 \mathrm{~ms}$ and a frame size of $5 \mathrm{~s}$, as we determined to be a reasonable time frame to detect single motility modes of distinct frequencies earlier. The following three pages show the different frequencies, inherent in the motility of a tumbling and a running cell, distinguished by the autocorrelation of their center of mass displacement.

Trypanosome motility patterns

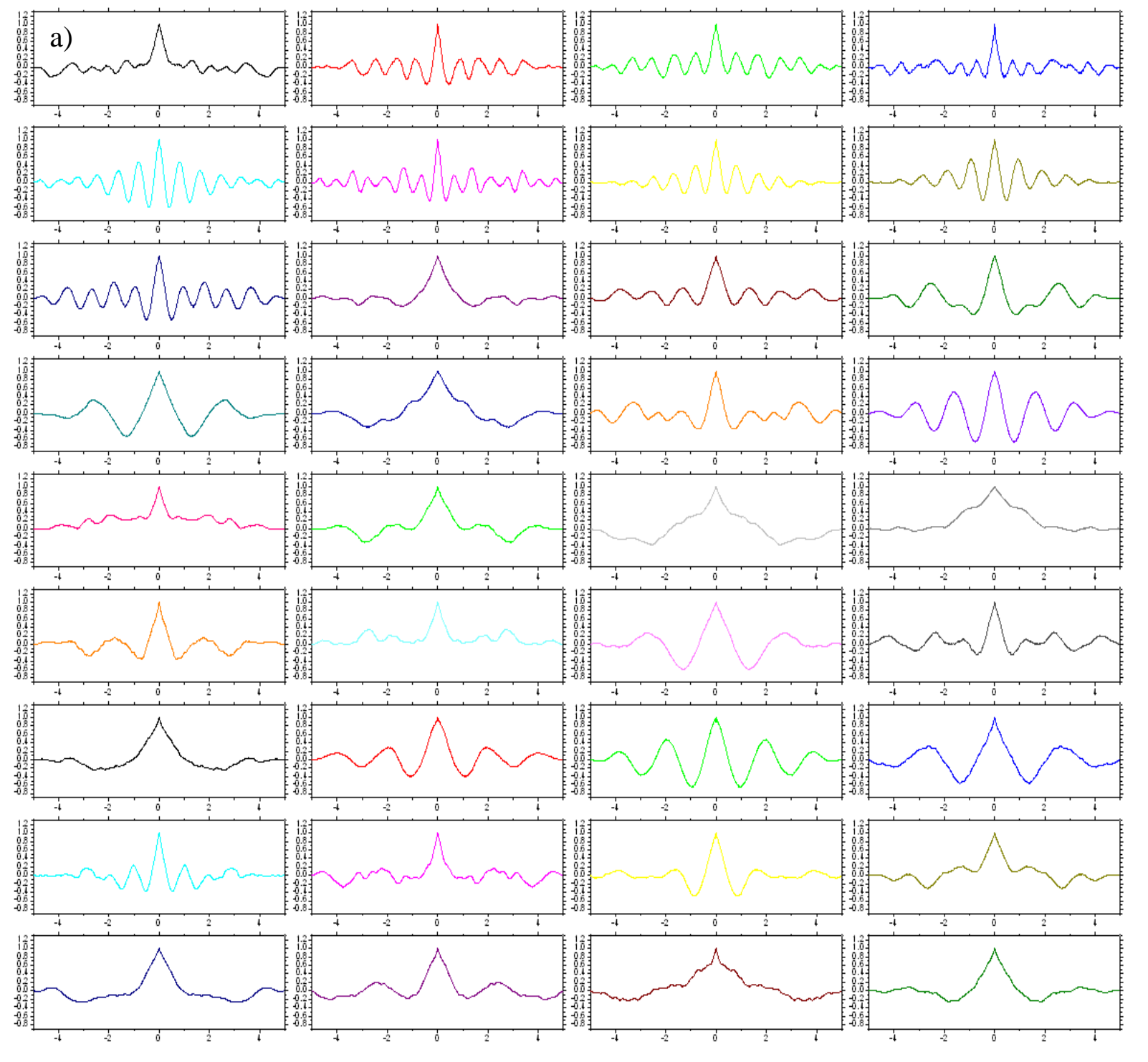




\section{MOTILITY OF TRYPANOSOMES}

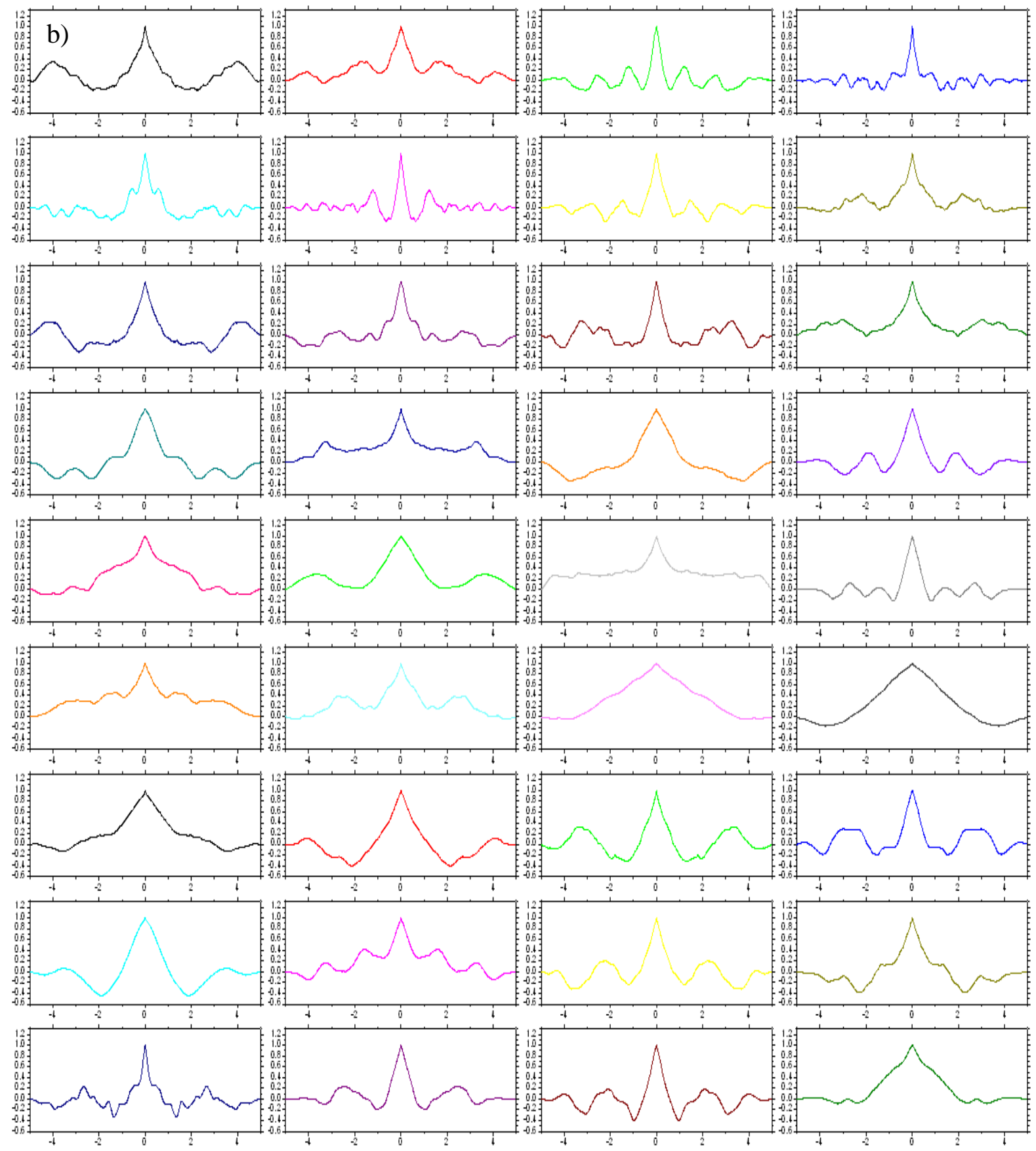

Fig. 4.19: Changes in cell motility over time. Every graph shows the autocorrelation function of x-axis center of mass displacement. The moving time frame is $5 \mathrm{~s}$ for each graph with a step size of $0.01 \mathrm{~s}$. Total time of observation is $180 \mathrm{~s}$. a) running cell; b) tumbling cell.

By looking at the plots, the changes in motility over time become obvious. The running trypanosome shows very regular motility, with a constant period of 1.67 rotations per second over $11 / 2$ minutes, typical for a persistent cell (S. Uppaluri). The motility then changes to 1.35 rotations per second. 
The comparison of the center of mass trace and it autocorrelation describes the effect of persistent frequencies on the displacement pattern. Reduced periodicity in motility leads to irregular center of mass displacement.

\section{running and tumbling motility patterns}
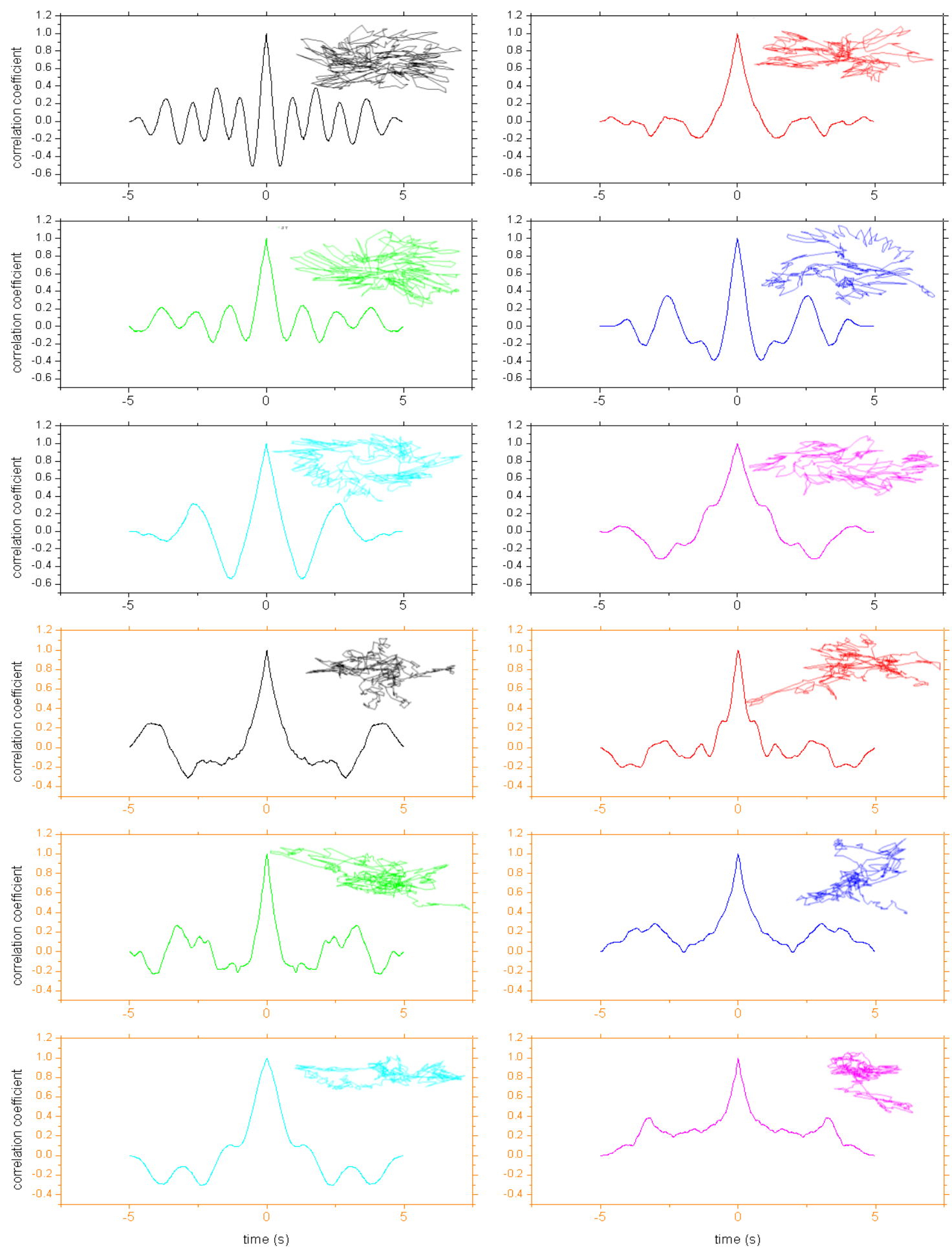

Fig. 4.20: Zoom into the first six plots of IFg. $\mathrm{X}$ a) and b) including the according xy-position traces for comparison. Black outline: running cell; orange outline: tumbling cell. 
The appearance of different prominent frequencies which we assign to overall rotational motility $f_{1}$ and flagellar beating $f_{3}$, differs for running and tumbling cells (Fig. 4.21)

Tumbling motility patterns

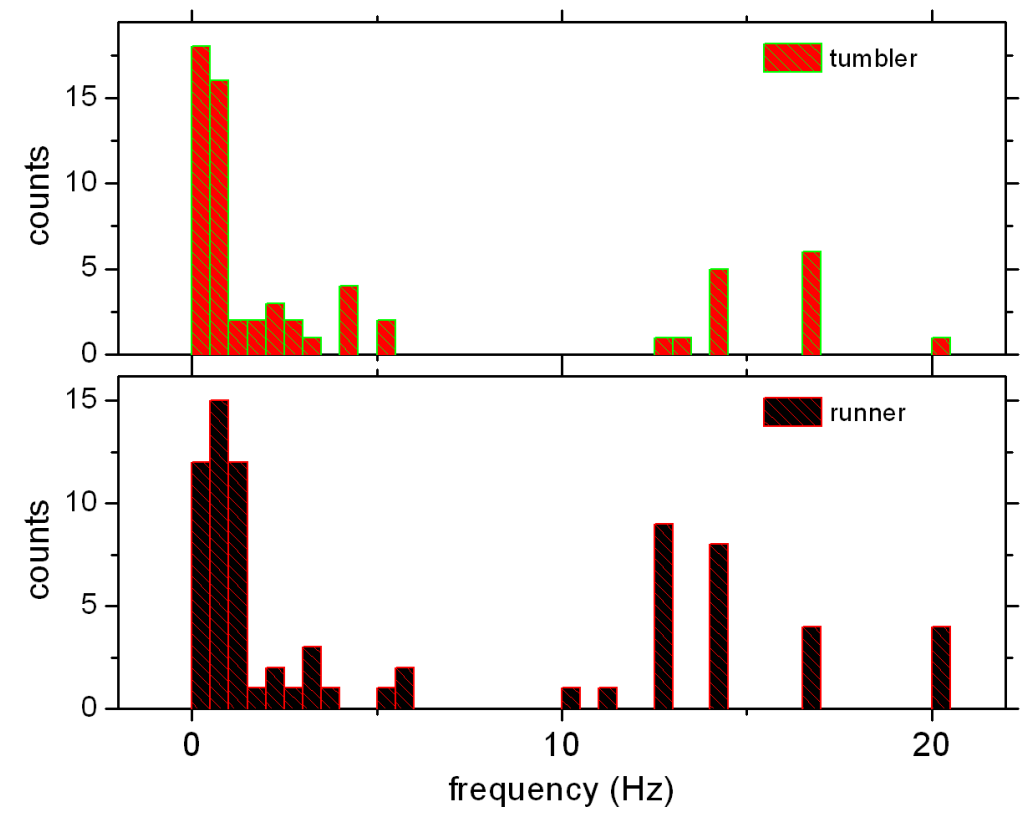

Fig. 4.21: Histogram of frequencies emerging from center of mass displacement autocorrelations of figure X. Black bars: running cell; orange bars tumbling cell.

Prominent peaks in the autocorrelation functions were found at mean frequencies of $f_{1}=1.3$ $\mathrm{Hz}$ and $f_{2}=14.6 \mathrm{~Hz}$ over $180 \mathrm{~s}$ for the running cell. The mean frequencies found for the tumbling cell were different with $f_{1}=1.5 \mathrm{~Hz}$ and $f_{2}=15.7 \mathrm{~Hz}$.

The number of rotations and flagellar beats for both kinds of cells is compared by the sum of frequencies obtained over 180s given by figures 4.22 .

sum of cell rotations $f_{1}$

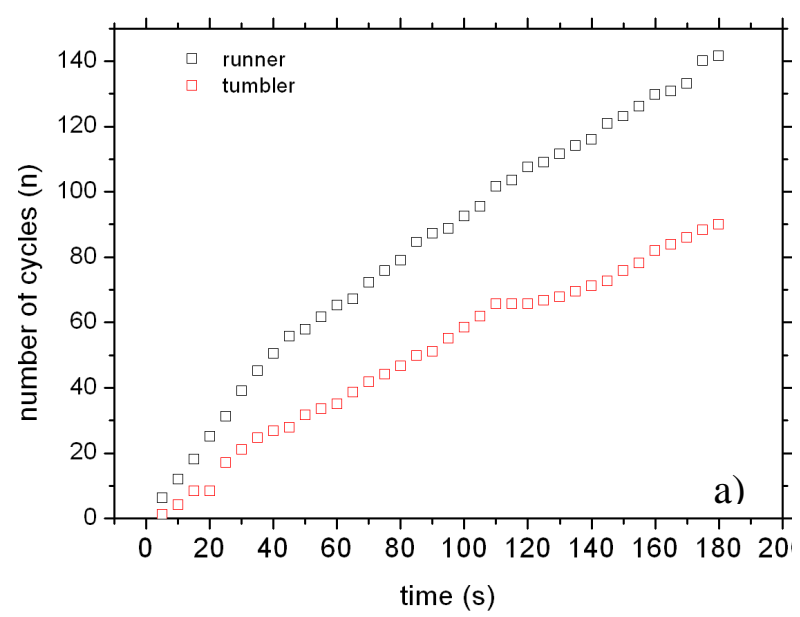

sum of flagellar beats $f_{2}$

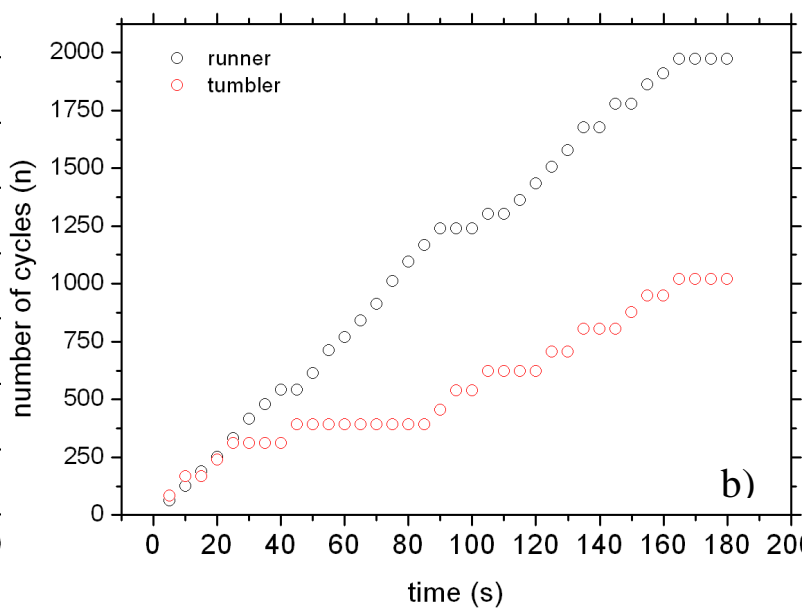

Fig. 4.22: Sum of trypanosome frequencies over 180 s. a) Sum of f1 rotations; b) Sum of f3 flagellar beats. 
Both the mean rotation and flagellar beating frequencies are slightly higher for tumbling cells, but they are less continuous and frequently interrupted by irregular movements (Fig. $4.19 \mathrm{~b}$ ) plot 6-7, 10-17; Fig. 4.20). These interruptions, most prominent in the flagellar beating frequencies $f_{2}$, result in tumbling motion, where force is still generated, but lacks persistence in periodicity.

Using the correlation analysis in this section, we were able to show the differences in rotational periodicity of different types of trypanosomes swimmers, while in an optical trap. We expect this rotational periodicity to be of direct consequence on the overall motility (directionality) of the trypanosome. 


\section{HYDRODYNAMIC SYNCHRONISATION OF TRYPANOSOMES}

\section{INTRODUCTION}

Hydrodynamic interactions have proven to be of crucial importance for life at low Reynolds numbers $(\underline{61}, \underline{62}, 63)$, as bigger life forms microorganisms are capable of fluid flow sensing and signalling, as well as swarming together in flocks, to improve propagation. Very recently, social motility has been reported for epimastigote trypanosomes in vitro. The epimastigote stage lives in the insect vector and has to migrate from the tsetse flies gut to the salivary gland in order to be transferred to a new host during the blood meal. These cells have been reported to build huge assemblies on semi-solid agarose surfaces and propagate directionally, comparable to pseudopodial motion observed for single cells and cell assemblies like fruiting bodies in Dictyostelium. We measured that cell doublets which have not yet divided can produce twice as much propagation force as a single cell (chapter 3.5). Moving in flocks to benefit propagation in the blood stream or into tissue may require concerted interaction of single cells in a fluid environment.

Hydrodynamic interactions of trypanosomes as a function of distance are analyzed in the following chapter using a dual optical trap setup with high speed imaging.

\section{Hydrodynamic interaction}

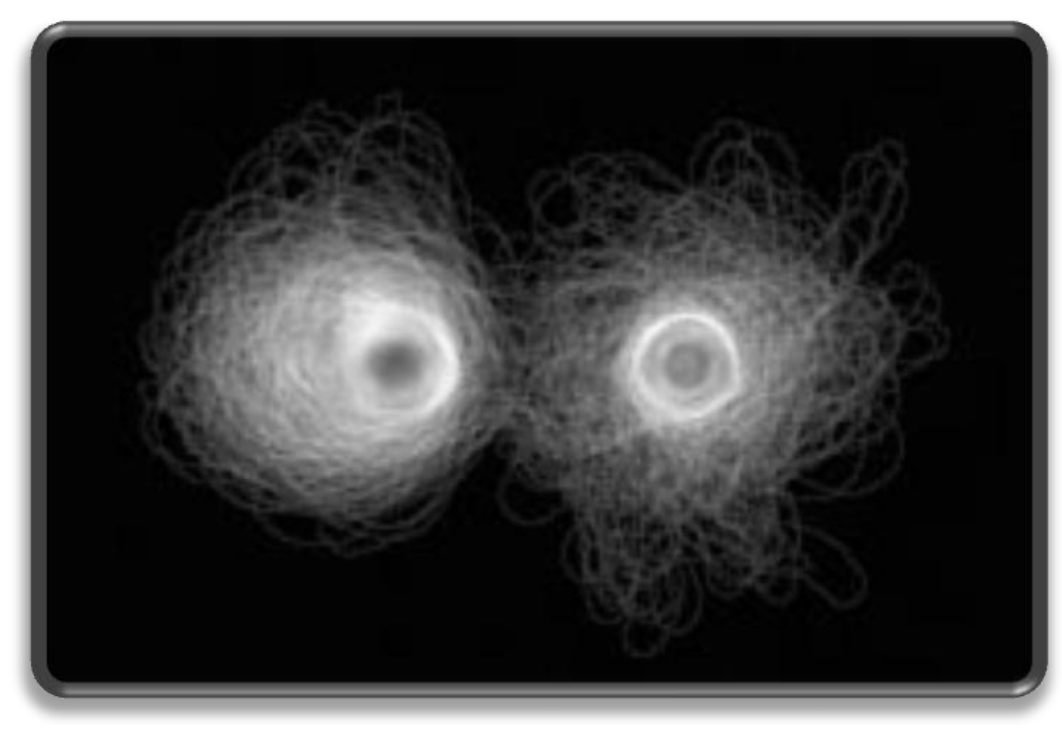

Fig. 4.23: Contour line overlay of two independently trapped trypanosomes at a distance of $10 \mu \mathrm{m}$. 


\section{EXPERIMENT}

The cells and microfluidic channels were prepared as described in chapter 2. Both optical traps were set to a power of $20 \mathrm{~mW}$ at the sample. The trapping distance was varied in $6 \mu \mathrm{m}$ steps from 24 to $6 \mu \mathrm{m}$. We analyzed five different cells for four distances to a total of $n=20$ cells. All cells were trapped for $30 \mathrm{~s}$ per distance, then the distance was changed in $10 \mathrm{~s}$ and recorded again, giving a total of $150 \mathrm{~s}$. Image processing was conducted as described in chapter 2.

\section{RESULTS AND DISCUSSION}

\section{HYDRODYNAMIC INTERACTION}

Changes and similarities in motion of two enclosing cells have been analyzed by center of mass displacements autocorrelation in $x$ - and $y$-axis direction (Fig. 4.24). However, the autocorrelation of the $x$-axis displacement show the same features as the autocorrelations of the cosine of XY displacement (Fig. 4.24b) which is used for further analysis.

$\mathrm{x}$-center of mass ac

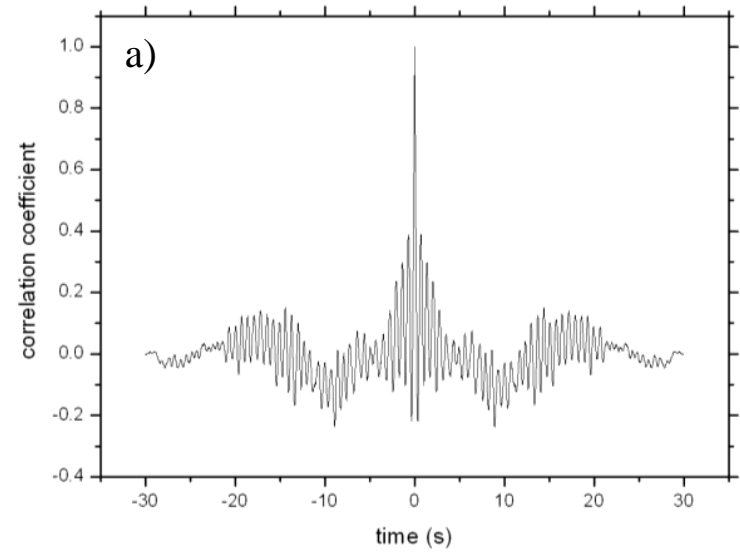

xy cosine ac

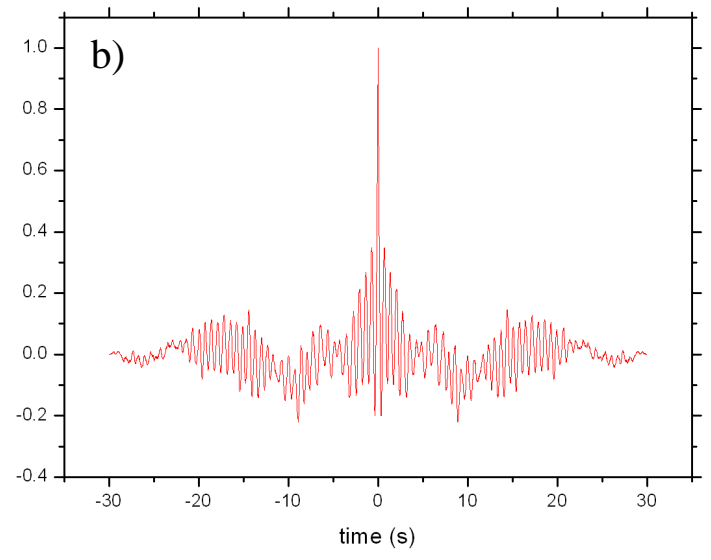

Fig. 4.24: a) X-axis displacement autocorrelation function of cell $2(6 \mu \mathrm{m})$. b) Cosine of xy center of mass displacement autocorrelation function of cell $2(6 \mu \mathrm{m})$. Black line: com; red line: cosine

The two cells in figure 4.25a show different motility patterns at a distance of $24 \mu \mathrm{m}$. Reducing the distance between the two trapped trypanosomes to $12 \mu \mathrm{m}$, swimmer 1 (black line) shows a different autocorrelation function than before: strong anti-correlations at a time span of $10 \mathrm{~s}$ can be found. Reducing the distance further to $6 \mu \mathrm{m}$, the autocorrelation function of swimmer 2 (red line) suddenly shows the same long time features as well, whilst the short time correlations are more pronounced in comparison to swimmer 1 . The evolution of the motility patterns in dependence of the distance indicates a crosstalk between the two cells. 


\section{Autocorrelations}
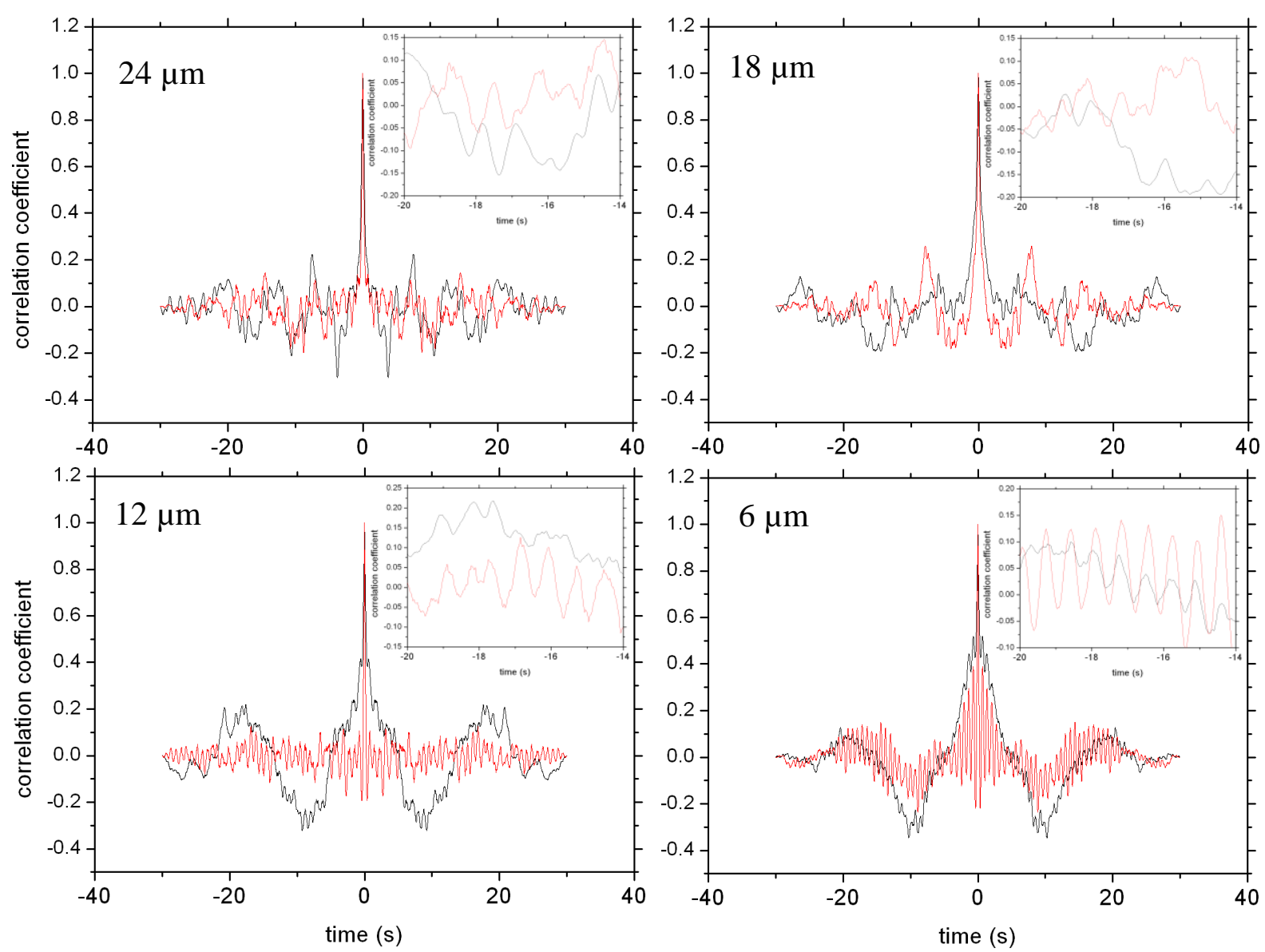

Fig. 4.25: $x$-axis displacement autocorrelation function of two independently trapped trypanosomes at different distances. Black line: swimmer 1; red line: swimmer 2. Inset plots: Zoom to the data.

In order to quantify this crosstalk between the two independently trapped trypanosomes, we calculated the crosscorrelation functions of the displacements between the swimmer 1 and swimmer 2.

\section{Crosscorrelations}
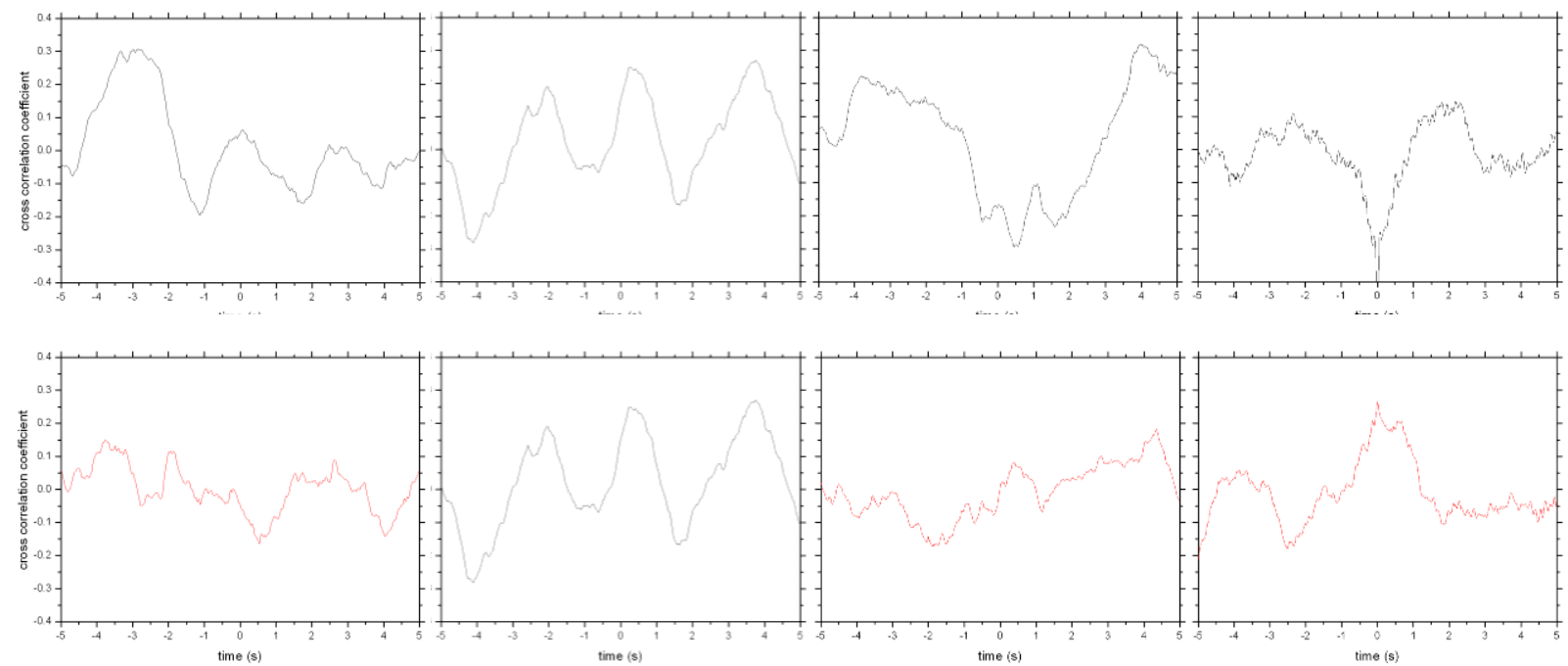

Fig. 4.26: Crosscorrelation functions of the displacements between swimmer 1 and swimmer 2 at different 
The $x$ - and $y$-axis displacement crosscorrelation functions of the trapped trypanosome pair over the trap distance are shown in figure 4.26. The average crosscorrelation coefficients of $x$ and $y$-axis displacements at $t=0 \mathrm{~s}$ versus the distance for 5 pairs of independently trapped trypanosomes are shown in figure 4.27. At distances $d \geq 12 \mu \mathrm{m}$, the crosscorrelation coefficients for the two displacements are close to 0 and do not show a specific tendency. However, at a distance of $6 \mu \mathrm{m}$, strong crosscorrelation can be observed. Whereas the $x$-axis displacements show a pronounced anti-correlation, the $x$-axis displacements indicate a positive correlation.

\section{Crosscorrelations}

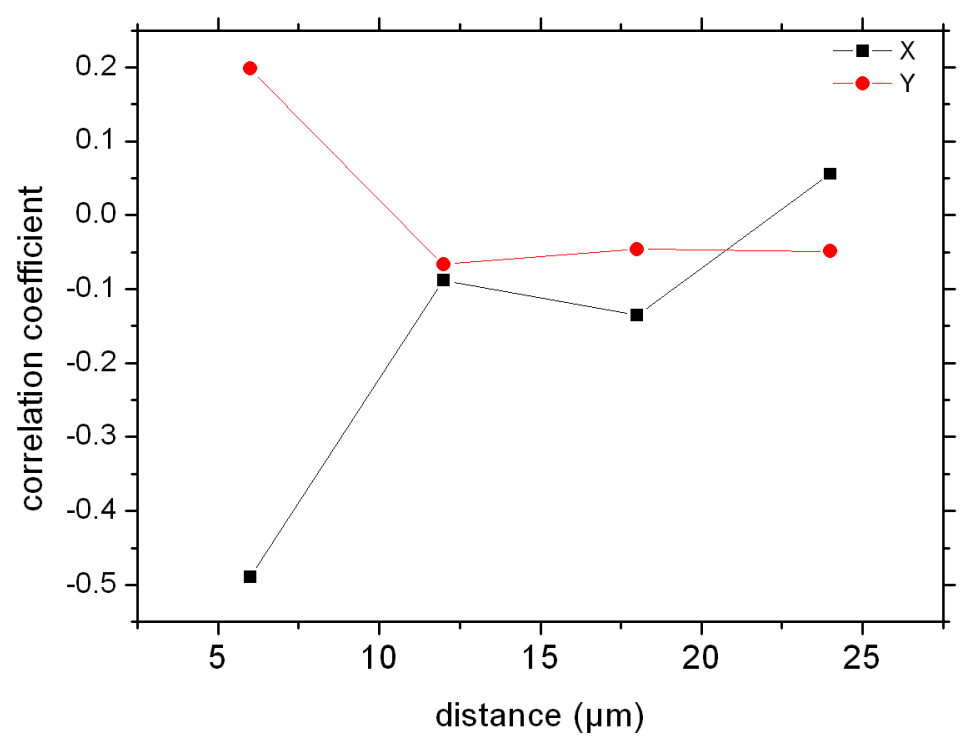

Fig. 4.27: Crosscorrelation coefficients of $x$ - and $y$-axis displacements at $t=0 \mathrm{~s}$ (average of for 5 pairs of independently trapped trypanosomes)

These results show that independently trapped trypanosomes interact over short distances of $D<1 / 2$ body length, due to hydrodynamic forces. Moreover, at close distances, the trypanosomes may correlate in a specific manner: one trypanosome is rotating clockwise the second one is rotating counter clockwise. 


\section{CHAPTER SUMMARY AND CONCLUSIONS}

The motivation of this thesis was to describe and understand the complexity of trypanosome motility. Combining optical trapping and microfluidic techniques with high speed microscopy, we developed a toolbox for a broad range of biophysical studies on trypanosomes.

We were able to manipulate trypanosomes within microfluidic environments using optical traps and measure the propagation forces of single cells. In doing so, we found that dividing cells which had already grown a second flagellum, can generate twice as much force as single cells with only one flagellum. Moreover, we found that those cells which tumble in open space are forced into directed propagation if confined in channels of only $2 \mu \mathrm{m}$ in width. These findings indicate that different motility modes might represent adaptations to the environmental needs of certain cell cycle stages, a dividing cell might be able to propagate faster than single cells and explore new habitats more rapidly, while a tumbling motion of single cells might be an adaption for migration trough dense tissues.

We were able to show for the first time that optical manipulation of trypanosomes is possible using focused laser diodes and that the preferred trapping locus is the basal body of the trypanosome body. Further, quantitative analysis of trypanosome trapping characteristics, made possible by automated image processing routines, allowed us to distinguish running cells from tumbling ones in terms of frequency, direction and velocity.

Precise control of a second optical trap in the system allowed for the measurement of the forces generated by the flagellar tip. Moreover, when both the optical traps were used to position two trypanosomes in close proximity, correlated motion, eventually leading to cell synchronisation, was seen. Hydrodynamic interactions of low Reynolds numbers swimmers such as sperm, and bacterial cells have been reported. The optical setup built herein, provides a platform for detailed studies of hydrodynamic interactions between single cell organisms.

As qualitative knowledge of biological processes emerges, it is becoming more and more evident that concurrent quantitative insight is required both to further our fundamental understanding of life at low Reynolds numbers and enable urgent medical research. The tools presented in this work provide a basis for future quantitative studies, not only for trypanosomes, but also for other unicellular organisms. 


\section{OUTLOOK}

Trypanosome swimming induces hydrodynamic drag on the surface of the cell to sweep away host antibodies, thus allowing them to evade immunological attacks by the host.

The usage and modification of different labelling techniques for trypanosomes allows for further studies, not only on trypanosomes in optical or spatial confinements, but also on the mechanism of antibody removal. In previous work by Engstler at al, antibody labelling was used to label the cell surface and observe the sweeping of the antibodies to the cell mouth. However, this traditional labelling method is limiting especially in the microfluidic environment. To this end, we have been able to develop a cell labelling method using Quantum dots. Quantum dots are much easier to handle, most importantly, because they do not bleach during fluorescence measurements. As seen in the figure below, quantum dots on the surface of the trypanosome are swept to the cell mouth, demonstrating that the quantum dots are a good model system to further study hydrodynamic drag of cell surface proteins.

\section{Qdot labeling}
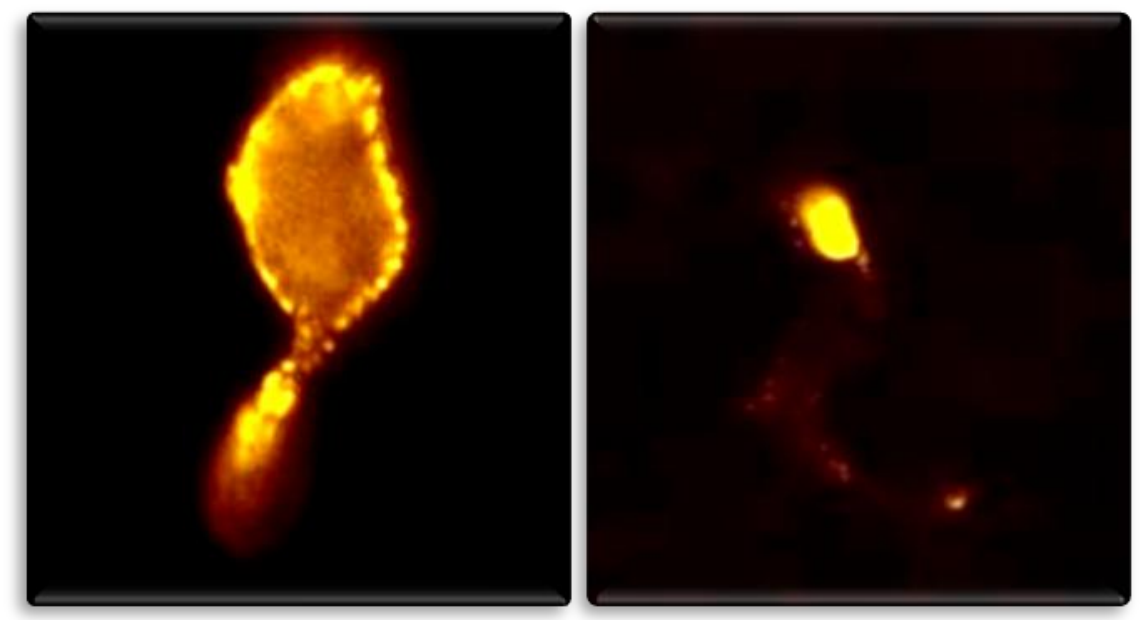

\section{Biotin Streptavidin Qdot labelled trypanosomes}

Furthermore, using our optical trapping toolbox in combination with advanced multi purpose microfluidic devices, we were ultimately able to covalently label VSG proteins with tracer particles to uncover the dynamics of antibody removal in realtime. The following figure gives the proof of principle for further experiments. 


\section{single particle stamping}

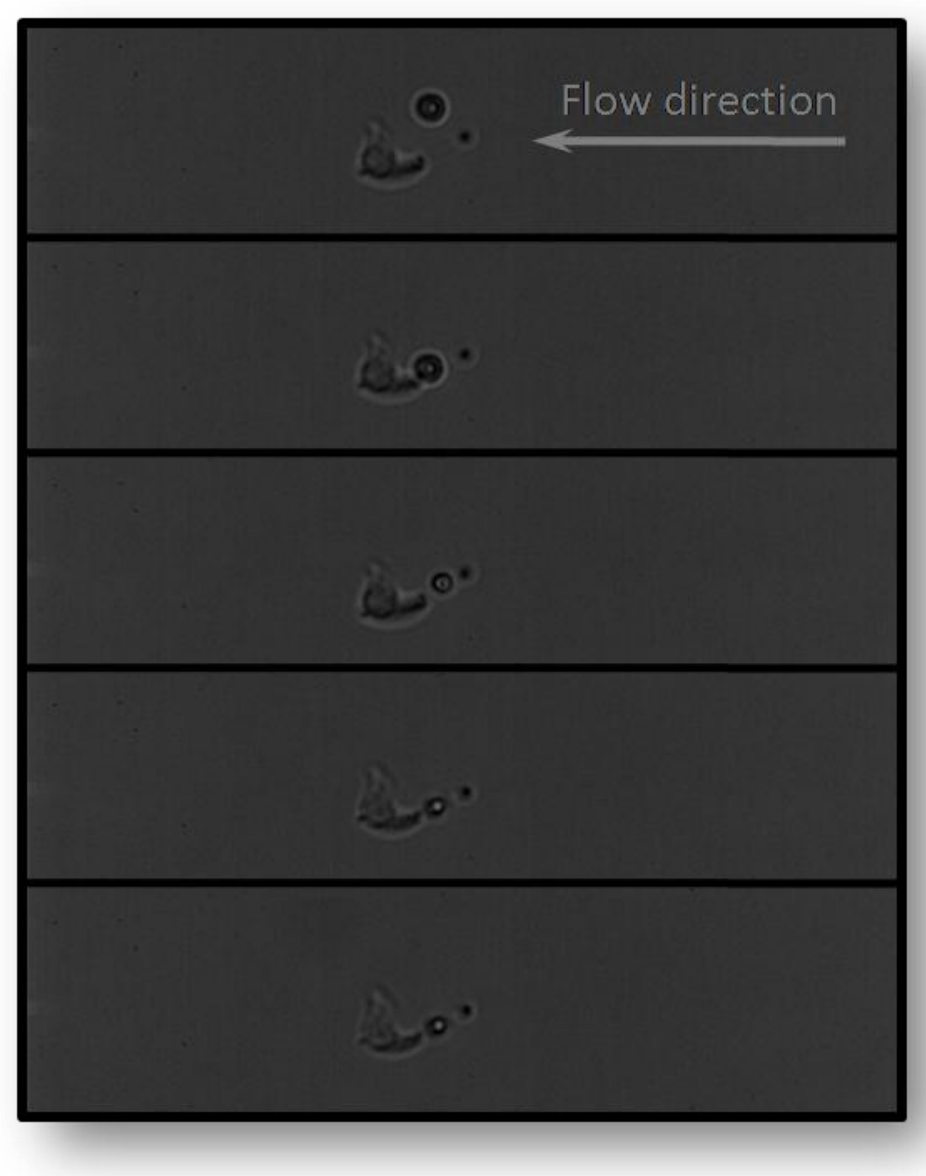

$\mathrm{COOH}$ polystyrene bead covalently coupeld to VSG surface proteins of a trypanosoma cell

This figure shows, how a tracer bead, being stamped on the surface of the cell via the second optical trap, can be observed. This demonstrates that our setup can be utilized towards real time studies of antibody removal from the cell surface. 


\begin{tabular}{|c|c|c|}
\hline INSTRUMENT & BRAND & MODEL \\
\hline MiCROSCOPE & OLYMPUS & BX61 \\
\hline MiCROSCOPE & OLYMPUS & IX81 \\
\hline MiCROSCOPE & ZEISS & AXIOVERT 135 \\
\hline MICROSCOPE & LEICA & SP2 \\
\hline MICROSCOPE & SELF CONSTRUCTION & LINOS MICROBANK \\
\hline INTERFEROMETER & VEECO & NT 1100 \\
\hline MICROMANIPULATOR & SUTTER & MP 285 \\
\hline CAMERA & $\mathrm{PCO}$ & SENSICAM QE \\
\hline CAMERA & $\mathrm{PCO}$ & PCO 1600 \\
\hline CAMERA & $\mathrm{PCO}$ & PIXELFLY QE \\
\hline CAMERA & VISION RESEARCH & PHANTOM MIRO 3 \\
\hline LASER & BLUESKY RESEARCH & MiNILASE MODULE \\
\hline LASER & РнотоР-SUWTECH & DPGL-2050 \\
\hline LASER DIODE & BLUESKY RESEARCH & VPSL-0808-200-X-5-B \\
\hline LASER DIODE & THORLABS & L980P300J \\
\hline LASER DIODE & SANYO & DL-7146-101S \\
\hline LASER DRIVER & WAVELENGTH ELECTRONIC & FL-593LD USB \\
\hline LASER DRIVER & ILX LIGHTWAVE & LDX 3500 \\
\hline POWER SUPPLY & VOLTCRAFT & DPS-4005PFC \\
\hline Light EmitTing Device & CREE & XPGWHT-L1-1T-R5 \\
\hline Light EmitTing Device & CREE & XPEGRN-L1 G4 Q4 \\
\hline Light EMItTing DEVICE & CREE & XP-G R5-7090-Q5- P4 \\
\hline
\end{tabular}




\section{MATERIALS}

\begin{tabular}{|c|c|c|}
\hline INSTRUMENT & BRAND & MODEL \\
\hline ACCUSTO OPTIC MODULATOR & GOUCH\&HOUSEGO & M080-1X-GHX \\
\hline AOM DRIVER & LANDWEHR & A341 \\
\hline PULSE GENERATOR & QUANTUM COMPOSERS & 9600 PLUS \\
\hline MASK ALIGNER & KARL SUSS MICROTEC & MJB3 UV 300/400 \\
\hline HOT PLATE & EUROTHERM CONTROLS & 2123 \\
\hline Hot Plate & EURÓTHERM CONTROLS & 2416 \\
\hline SPINCOATER & COOKSON & SCS G3P-8 \\
\hline PLASMA CLEANER & HARRICK SCIENTIFIC LTD & HARRICK PDC-32G \\
\hline VACUUM PUMP & ILMVAC GMBH & MPC $201 \mathrm{~T}$ \\
\hline VACUUM CHAMBER & NALGENE & NG 50 \\
\hline CHROMIUM MASK & MLC & QUARTZ GLASS \\
\hline PLASTIC MASK & JD PHOTO TOOLS LTD & POLYMER INK COATED \\
\hline STERILE FLOW BENCH & HERAEUS & HERA SAFE KS 18 \\
\hline INCUBATOR & HERAEUS & HERA CELl 150 \\
\hline NITROGEN CHAMBER & AIR LIQUIDE & ARPEGE 40 \\
\hline REFRIGERATOR & GFL & 6483 \\
\hline WATER SUPPLY & MILLIPORE & MiLli-Q A10 \\
\hline CENTRIFUGE & HEAREUS & LABFUGE 400 \\
\hline CENTRIFUGE & EPPENDORF & $5415 \mathrm{D}$ \\
\hline TEMPERATURE CONTROLLER & MCSHANE INC & MC 378 \\
\hline TEMPERATUR SENSOR & MCSHANE INC & TS $136-170$ \\
\hline PELTIER ELEMENT & TELEMETER & TB-99-1.4-1.05 \\
\hline
\end{tabular}




\section{MATERIALS}

\begin{tabular}{|c|c|c|}
\hline INSTRUMENT & BRAND & MODEL \\
\hline MICRO PUMP & MPI DS & U. KRAFT, T. PFOHL \\
\hline MICRO PUMP & KD SCIENTIFIC & 100 \\
\hline MICRO PUMP & CETONI & NEMESYS \\
\hline MICRO PUMP & HARVARD APPARATUS & \\
\hline MICRO PUMP & LAMBDA & VITFIT \\
\hline OBJECTIVE & ZEISS & ECPlan NEOFLUAR 100X/1.3 \\
\hline LENS & LIGHTPATH & GPX-10-30-DB5 GRADIUM \\
\hline LENS & LINOS & ACHROMAT F 200 \\
\hline LENS & LiNOS & ACHROMAT F 160 \\
\hline BEAMSPLITTER & SEMROCK & HC 670/SP \\
\hline EMITTER & SEMROCK & HC 680/SP \\
\hline AG MIRROR & LINOS & F21-005 \\
\hline POLARIZER & THORLABS & CM1-PBS252 \\
\hline BEAMSPLITTER & CHROMA & Z 532 RDC \\
\hline EMITTER & SEMROCK & HC 528/38 \\
\hline CRYOBOX & STRATAGENE & STRATA COOLER \\
\hline POWERMETER & THORLABS & PD 200 \\
\hline POWERMETER & MELLES GRIOT & 13 PEM 001/J \\
\hline
\end{tabular}




\section{MATERIALS}

\begin{tabular}{|c|c|c|}
\hline SOFTWARE & BRAND & MODEL \\
\hline OPERATING SYSTEM & MiCROSOFT & WINDOWS XP \\
\hline OFFICE SOFTWARE & MiCROSOFT & OFFICE 2007 \\
\hline MATH SOFTWARE & ORIGINLAB COOPERATION & ORIGIN 8.1 \\
\hline MATH SOFTWARE & WOLFRAM RESEARCH & MATHEMATICA 6 \\
\hline MATH SOFTWARE & MATHWORKS INC. & MATLAB R2009A \\
\hline IMAGE SOFTWARE & IMAGEJ & IMAGEJ 1.44 \\
\hline IMAGE SOFTWARE & MEDIA CYBERNETICS & IMAGE-PRO-PLUS \\
\hline SKETCH SOFTWARE & AUTODESK & AUTOCAD 2005 \\
\hline PAINT SOFTWARE & COREL COOPERATION & COREL DRAW 12 \\
\hline PHOTO SOFTWARE & ADOBE SYSTEM CORP. & ADOBE PHOTOSHOP CS \\
\hline CAMERA SOFTWARE & $\mathrm{PCO}$ & CAMWARE 2.0 \\
\hline CAMERA SOFTWARE & VISION RESEARCH & PHANTOM CAMERA CONTROL \\
\hline MICROSCOPE SOFTWARE & OLYMPUS SOFT IMAGING & $\mathrm{CELL}^{\wedge} \mathrm{R} 3.3$ \\
\hline MicROSCOPE SOFTWARE & LEICA & LCS \\
\hline INTERFACE SOFTWARE & NATIONAL INSTRUMENTS & LABVIEW \\
\hline
\end{tabular}




\begin{tabular}{|c|c|c|}
\hline Chemicals & BRAND & CHEMICAL \\
\hline POLYMER & DOW CORNING & SYLGARD 184 \\
\hline PHOTORESIST & MicroCheM & SU8 50-100 \\
\hline PHOTORESIST & MicroCheM & SU8 2000 \\
\hline PHOTORESIST & MicroCheM & SU8 3000 \\
\hline MEdium & INVITROGEN & IMDM (42200-030) \\
\hline MEDIUM & INVITROGEN & FCS (10270-106) \\
\hline ANTIBIOTIC & INVITROGEN & PENICILLIN STREPTOMYCIN \\
\hline AMINO ACID & SIGMA ALDRICH & CYSTEIN (C7352) \\
\hline AMINO ACID & SigMA ALDRICH & THYMiDIN (T-1895) \\
\hline AMINO ACID & SIGMA ALDRICH & HYPOXANTHIN (H9636) \\
\hline REAGENT & SERVA & BATHOCUPROIN (14470.03) \\
\hline REAGENT & Rотн & $\begin{array}{l}\text { B-MERCAPTOETHANOL } \\
(4227.3)\end{array}$ \\
\hline REAGENT & PIERCE & $\begin{array}{l}\text { SULFO-NHS-BIOTIN } \\
(21217)\end{array}$ \\
\hline REAGENT & PIERCE & $\begin{array}{l}\text { SULFO-NHS-LC-LC-BIOTIN } \\
\text { (21338) }\end{array}$ \\
\hline REAGENT & PIERCE & EDC (22980) \\
\hline REAGENT & PIERCE & DTSSP (21578) \\
\hline DYE PARTICLES & POLYSCIENCES INC. & POLYBEAD MicROSPHERES \\
\hline DYE PARTICLES & Molecular Probes & $\begin{array}{l}\text { STREPTAVIDIN } 585 \text { QDOTS } \\
\text { (Q10101MP) }\end{array}$ \\
\hline DYE PARTICLES & Molecular Probes & $\begin{array}{l}\text { QTRACKER } 585 \text { QDOTS } \\
\text { (Q25011MP) }\end{array}$ \\
\hline DYE PARTICLES & MOLECULAR PROBES & $\begin{array}{l}\text { Carboxyl } 605 \text { ITK Qdot } \\
\text { (Q21301MP) }\end{array}$ \\
\hline
\end{tabular}




\section{MATERIALS}

\begin{tabular}{lll} 
CHEMICAL TYPE & BRAND & CHEMICAL \\
\hline DYE PARTICLES & POLYSCIENCES INC. & FlUOSPHERES \\
\hline DYE & PIERCE & NHS-RHODAMINE (46102) \\
DYE & PIERCE & AMCA-SULFO-NHS (33010) \\
\hline DYE & MOLECULAR PROBES & HOECHST (33342) \\
DYE & MOLECULAR PROBES & DAPI (D-1306)
\end{tabular}




\section{REFERENCES}

1. D. Steverding. The history of African trypanosomiasis.

Parasites \& Vectors, 2008; 1-3.

2. Stich, D. Steverding. Die Rückkehr einer Seuche.

Biologie in unserer Zeit, 32.

3. Stich, P. M. Abel, S. Krishna. Human African trypanosomiasis.

British Medical Journal, 2002; 27; 203:206.

4. D. Steverding. The development of drugs for treatment of sleeping sickness: a historical review.

ParasitVectors, 2010; 10, 3:15.

5. W. Gibson. Resolution of the species problem in African trypanosomes. Int J Parasitol., 2007; 37(8-9):829-38.

6. E.M.Fèvre, K. Picozzi, J. Jannin, S.C. Welburn, I. Maudlin. Human African trypanosomiasis: Epidemiology and control.

Adv Parasitol, 2006; 61:167-221.

7. L. De-Hua, H. Hashimi, L. Zhao-Rong Lun, A.J. Francisco, J. Lukeš. Adaptations of Trypanosoma brucei to gradual loss of kinetoplast DNA: Trypanosoma equiperdum and Trypanosoma evansi are petite mutants of $T$. brucei. PNAS, 2003; 105, no. 6.

8. W. De Souza. Basic cell biology of Trypanosoma cruzi. Curr Pharm, 2002; 8(4):269-85.

9. J. E. Donelson, M. J. Gardner, N. M. El-Sayed. Commentary: More surprises from Kinetoplastida.

Proc. Natl. Acad. Sci. USA, 1999, Vol. 96, 2579-2581.

10. Tait. Evidence for diploidy and mating in trypanosomes.

Nature, 1980; 287, 536 - 538. 
11. M. Hope, A. MacLeod, V. Leech, S. Melville, J. Sasse, A. Tait, C.M. Turner. Analysis of ploidy (in megabase chromosomes) in Trypanosoma brucei after genetic exchange.

MolBiochem Parasitol. 1999; 25;104(1):1-9.

12. El-Sayed NM, Hegde P, Quackenbush J, Melville SE, Donelson JE. The African trypanosome genome.

Int J Parasitol, 2000, 10; 30(4):329-45.

13. Piet Borst. Antigenic Variation and Allelic Exclusion.

Cell, 2002, 109, 5-8.

14. M. Engstler, L. Thilo, F. Weise, C. G. Grünfelder, H. Schwarz, M. Boshart and P. Overath. Kinetics of endocytosis and recycling of the GPI-anchored variant surface glycoprotein in Trypanosoma brucei.

Journal of Cell Science, 2004; 117, 1105-1115.

15. K. Stuart,T.E. Allen, M.L. Kable, S. Lawson. Kinetoplastid RNA editing: complexes and catalysts.

Curr Opin Chem Biol, 1997;1(3):340-6.

16. Katherine S. Ralston, Zakayi P. Kabututu, J. H. Melehani, M. Oberholzer and K. L. Hill. The Trypanosoma brucei Flagellum: Moving Parasites in New Directions. Annu. Rev. Microbiol., 2009; 63:335-62.

17. Lodish, Berk, Matsudaira, Kaiser, Krieger, Scott, Zipursky, and Darnell. Molecular Cell Biology. Molecular Cell Biology.

W. H. Freeman, 2005; $5^{\text {th }}$ edition.

18. J. R. Kardon amd D. R. Vale. Regulators of the cytoplasmic dynein motor. Nature Reviews Molecular Cell Biology, 2009; 10, 854-865.

19. Y. Xia and G. M. Whitesides. Soft Lithography. Annu. Rev. Mater. Sci., 1998; 28:153-84.

20. D. C. Duffy, J. C. McDonald, O. J. A. Schueller, and G. M. Whitesides. Rapid Prototyping of Microfluidic Systems in Poly(dimethylsiloxane).

Anal. Chem., 1998; 70, 49744984. 
21. H. Makamba, J. H. Kim, K. Lim, N. Park, J. H. Hahn. Surface modification of poly(dimethylsiloxane) microchannels.

Electrophoresis, 2003, 24, 3607-3619.

22.S. Lee and J.Vörös. An aqueous-based surface modification of poly(dimethylsiloxane) with poly(ethylene glycol) to prevent biofouling.

Langmuir, 2005; 21, 1195711962.

23. J. Kepler. De cometis libelli tres, I. Astronomicvs, theoremata continens de motu cometarum.

Avgvst Vindelicorvm, 1619. 11.

24. A. Ashkin and J. M. Dziedzic. Optical Levitation by Radiation Pressure. Applied Physics Letter, 1971; Vol.19; 8.

25. Ashkin, J. M. Dziedzic, J. E. Bjorkholm, and Steven Chu. Observation of a singlebeam gradient force optical trap for dielectric particles.

Optics Letters, 1986; Vol. 11; No. 5.

26. D. G. Grier. A Revolution in Optical Manipulation.

Nature, 2003; 424, 810-816.

27. R. Paschotta. Encyclopedia of Laser Physics and Technology. Wiley-VCH, 2008, 1st edition.

28. KC Neuman, EH Chadd, GF Liou, K Bergman, and SM Block. Characterization of photodamage to escherichia coli in optical traps.

Biophys J., 1999; 77(5): 2856-2863.

29. H Liang, K T Vu, P Krishnan, T C Trang, D Shin, S Kimel, and M W Berns. Wavelength dependence of cell cloning efficiency after optical trapping. Biophys J., 1996; 70(3): 1529-1533.

30. W. Singer, S. Bernet, N. Hecker and M. Ritsch-Marte. Three-dimensional force calibration of optical tweezers.

Journal of modern optics, 2000, Vol. 47, No. 14/15, 29212931.

31. Wilhelm Burger and Mark J. Burge. Digital Image Processing: An Algorithmic Introduction using Java.

Springer, $1^{\text {st }}$ edition, 2008. 
32. A Ashkin and JM Dziedzic. Optical trapping and manipulation of viruses and bacteria.

Science, 1987; Vol. 235 No. 4795 pp. 1517-1520.

33. W. de Souza. An introduction to the structural organization of parasitic protozoa. Curr Pharm, 2008; 14(9):822-38.

34. J. Guck, S. Schinkinger, B. Lincoln, F. Wottawah, S. Ebert, M. Romeyke, D. Lenz, H. M. Erickson, R. Ananthakrishnan, D. Mitchell, J Käs, S. Ulvick and C. Bilby. Optical Deformability as an Inherent Cell Marker for Testing Malignant Transformation and Metastatic Competence.

Biophysical Journal, 2005; Vol.88, 3689-3698.

35. J. M. Nascimento, E. L. Botvinick, L.Z. Shi. Analysis of sperm motility using optical tweezers.

J. Biomed. Opt., 2006; Vol. 11.

36. W. de Souza. An introduction to the structural organization of parasitic protozoa. Curr Pharm, 2008;14(9):822-38.

37. J. G. Torre and M. C. L. Martinez. Dimensions of Short, Rodlike Macromolecules from Translational and Rotational Diffusion Coefficients. Study of the Gramicidin Dimer.

Biopolymers, 1984; Vol. 23,611-615.

38. Cantor CR and Schimmel. Chemistry Part II. Techniques for the study of biological structure and function.

W. H. Freeman, p. 561-562.

39. M. Engstler, T. Pfohl, S. Herminghaus, Michael Boshart, G. Wiegertjes; N. Heddergott, P. Overath. Hydrodynamic Flow-Mediated Protein Sorting on the Cell Surface of Trypanosomes.

Cell, 2007; Vol131, 505-515.

40. M. Parsons, B. Nielsen. Active transport of 2-deoxy-D-glucose in Trypanosoma brucei procyclic forms.

Mol Biochem Parasitol., 1990;42(2):197-203.

41. S. Uppaluri, J. Nagler,E. Stellamanns, N. Heddergott,M. Ensgtler,T. Pfohl. Impact of Microscopic Motility Schemes on the Overall Swimming Behavior of Parasites. Biophysical Journal, 2010; Vol. 98, pp. 416a-416a. 
42. X. Ma, JunQLu, R S. Brock, K. M Jacobs, P. Yang and Xin-Hua Hu. Determination of complex refractive index of polystyrene microspheres from 370 to $1610 \mathrm{~nm}$. Phys. Med. Biol., 2003; 48; 4165-4172.

43. J. Guck, R. Ananthakrishnan1, T. J. Moon, C. C. Cunningham, and J. Käs. Optical Deformability of Soft Biological Dielectrics.

Phys. Rev. Lett., 2000 84, 5451-5454.

44. I. Kariv and P.J. Marchand. Optical Forces for Noninvasive Cellular Analysis. Applied Optics, 2003; Vol. 42, pp. 5765-5773.

45. M. Hazewinkeluop, Encyclopaedia of Mathematics. Springer Verlag, 2002.

46. D. Watson, N. Hagen, J. Diver, P. Marchand, and M. Chachisvilis. Elastic Light Scattering from Single Cells: Orientational Dynamics in Optical Trap. Biophys J. 2004 August; 87(2): 1298-1306.

47. Michael T. Madigan, John M. Martinko. Biology of Microorganisms. Pearson Prentice Hall, $11^{\text {th }}$ Edition, 2006.

48. Overath P, Engstler M. Endocytosis, membrane recycling and sorting of GPIanchored proteins: Trypanosoma brucei as a model system.

Mol Microbiol. 2004 Aug; 53(3):735-44.

49. Maria E. Bulina, Konstantin A. Lukyanov, Olga V. Britanova, Daria Onichtchouk, Sergey Lukyanov and Dmitriy M. Chudakov. Chromophore-assisted light inactivation (CALI) using the phototoxic fluorescent protein KillerRed Nature Protocols 2006 Aug; 947 - 953

50. E. J. G. Peterman, F. Gittes and C. F. Schmidt. Laser-Induced Heating in Optical Traps.

Biophys J., 2003, 84(2): 1308-1316.

51. S.P. Gross. Application of optical traps in vivo. Methods Enzymol. 2003;361:162-74.

52. E.M. Purcell. Life at Low Reynolds Number. American Journal of Physics, 1976, Vol 45, 3-11. 
53. L.W. Janson, D.L. Taylor. In vitro models of tail contraction and cytoplasmic streaming in amoeboid cells.

J Cell Biol. 1993 Oct;123(2):345-56.

54. R. J. Eddy, L.M. Pierini, F. Matsumura, F.R. Maxfield. Ca2+-dependent myosin II activation is required for uropod retraction during neutrophil migration.

J Cell Sci., 2000;113:1287-98.

55. Y. Fukui, T. Kitanishi-Yumura and S.Yumura. Myosin II-independent F-actin flow contributes to cell locomotion in Dictyostelium.

Journal of Cell Science, Vol 112, 877-886.

56. T.M. Svitkina, A.B. Verkhovsky, K.M. McQuade, G.G. Boris. Analysis of the actinmyosin II system in fish epidermal keratocytes: mechanism of cell body translocation.

J Cell Biol., 1997;139(2):397-415.

57. Rodríguez J, Lopez M, Thayer M, Zhao Y, Oberholzer M, Chang D, Kisalu N, Penichet M, Helguera G, Bruinsma R, Hill K, Miao J. Propulsion of African trypanosomes is driven by bihelical waves with alternating chirality separated by kinks.

PNAS, 2009; vol. 106, no. 46.

58. P. J. Walker. Organization of Function in Trypanosome Flagella.

Nature , 1961; 189, 1017 - 1018.

59. K. L. Hill. Biology and Mechanism of Trypanosome Cell Motility.

Eukaryotic Cell, 2003, Vol. 2, p. 200-208.

60. M. Parsons.Glycosomes: parasites and the divergence of peroxisomal purpose. Mol Microbiol. 2004 Aug;53(3):717-24.

61. M. Oberholzer, M. A. Lopez, B. T. McLelland, K. L. Hill, Social Motility in African Trypanosomes.

PLoS Pathog, 2010; 6,(1).

62. C. Wollin, H. Stark. Metachronal waves in a chain of rowers with hydrodynamic interactions. arXiv:1012.0164.

63. C. J. Ingham and B. J. Eshel. Swarming and complex pattern formation in Paenibacillus vortex studied by imaging and tracking cells BMC Microbiol., 2008; 8: 36. 


\section{DANKE}

Mein herzlicher Dank gilt Prof. Dr.Thomas Pfohl für die sehr gute fachliche Betreung und die Unterstützung dieser Arbeit in jeder Hinsicht. Die letzten 4 Jahre mit ihm und seiner Arbeitsgruppe waren die glücklichste und anregendste Zeit meines bisherigen wissenschaftlichen Lebens.

Weiterhin danke ich Prof. Dr. Herminghaus für die Ermöglichung dieser Arbeit an diesem schönen Institut, Prof. Dr. Jörg Enderlein, Prof. Dr. Marc Timme sowie Prof. Dr. Eberhard Bodenschatz für ihre Bereitschaft zur Begutachtung der vorliegenden Arbeit.

Des Weiteren möchte ich mich für die gute Zusammenarbeit mit Prof. Dr. Markus Engstler und Niko Heddergott bedanken, ohne die wir sicher nie zu den Trypanosomen gekommen wären.

Insbesondere möchte ich mich auch bei meinen Kollegen und Freunden Sravanti Uppaluri, Carsten Grabow, Katharina Junghans, Meike, Kris Hantke, Udo Krafft, Paul Steffen, Christoph Gögelein, Shashi Thutupalli, Semra Ötztürk, Dominik Michler, Dagmar Steinhauser, Sarah Köster, Rolf Dootz, Heather Evans, Markus Schwamberger, Wolf Keiderling, Anupam Sengupta, Klaus Röller, Jan Ohle Claussen, Quentin Brosseau, Karthik Peddireddy, Sonia Utermann, Ciro Semprebon, Martha Sanchez de la Lama, Thomas Eggers, letztendlich dem ganzen Institut für ihre Unterstützung und die schönen Zeiten bedanken.

Nicht zu vergessen auch die organisatorische Unterstützung des GGNB Büros, insbesondere durch Antje Erdamnn und Prof. Dr. Grubmüller. Ohne das „Physics of Biological and Complex Systems“" Programm wäre eine diese interdisziplinäre Arbeit an der Uni Göttingen nur schwer möglich gewesen.

Sehr wichtig für das experimentelle Gelingen dieser Arbeit war die hervorragende technische Unterstützung unserer Institutswerkstätten, vor allem auch Herr Hesse durch seine ausbildende Tätigkeit! Von gleicher Wichtigkeit ist hierbei die Arbeit der Verwaltung, deren stille Tätigkeit im Hintergrund letztendlich das Institut als Einheit trägt.

Einen herzlichen Dank auch an meine Freunde aus der Heimat, sowie den hoods, deren Zurede und Optimismus dem Gelingen dieser Arbeit nicht unwesentlich beigetragen hat.

Insbesondere möchte ich meinen Eltern und Geschwistern für ihr Vertrauen und die Tatkräftige Unterstützung danken. Ihnen ist diese Arbeit gewidmet. 


\begin{tabular}{|c|c|}
\hline $\begin{array}{l}\text { ERIC STELLAMANNS } \\
\text { GEB. AM 19.08.1975 } \\
\text { IN LÜNEBURG } \\
\text { LEDIG }\end{array}$ & CURRICULUM VITAE \\
\hline $\begin{array}{l}\text { DOKTORARBEIT } \\
07.2006 \text { - HEUTE }\end{array}$ & $\begin{array}{l}\text { DOKTORARBEIT AM MAX PLANCK INSTITUT FÜR DYNAMIK UND } \\
\text { SELBSTORGANISATION IM RAHMEN DER „INTERNATIONAL MAX } \\
\text { PLANCK RESEARCH SCHOOL FOR PHYSICS OF BIOLOGICAL AND } \\
\text { COMPLEX SYSTEMS“ } \\
\text { THEMA DER DOKTORARBEIT: } \\
\text { „CELL MOTILITY AND PROTEIN DYNAMICS IN THE MICROFLOW” } \\
\text { EINGESCHRIEBEN AN DEN } \\
\text { MATHEMATISCH-NATURWISSENSCHAFTLICHEN FAKULTÄTEN } \\
\text { DER GEORG-AUGUST-UNIVERSITÄT ZU GÖTTINGEN }\end{array}$ \\
\hline $\begin{array}{l}\text { STUDIUM } \\
10.1998-09.2005\end{array}$ & 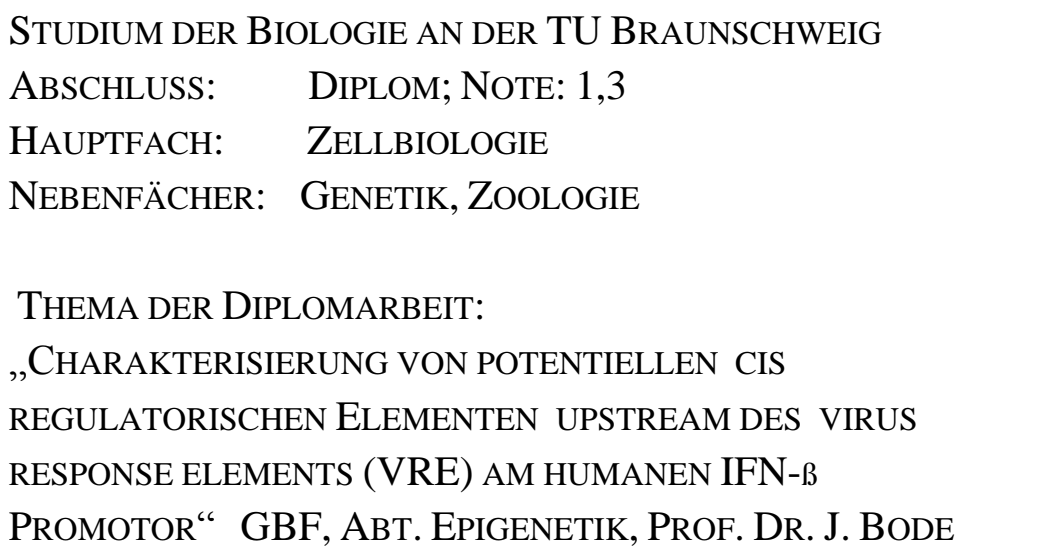 \\
\hline $10.1997-03.1998$ & STUDIUM DER PHYSIK AN DER UNIVERSITÄT HAMBURG \\
\hline $\begin{array}{l}\text { WEHRDIENST } \\
07.1996-04.1997\end{array}$ & $\begin{array}{l}\text { GRUNDWEHRDIENST IM 3. STABS- UND FERNMELDE- } \\
\text { BATTALION } 1 \text { IN ROTENBURG A. D. WÜMME }\end{array}$ \\
\hline $\begin{array}{l}\text { SCHULABSCHLUSS } \\
24.05 .1996\end{array}$ & $\begin{array}{l}\text { ABITUR AM BERNHARD RIEMANN GYMNASIUM } \\
\text { SCHARNEBECK }\end{array}$ \\
\hline
\end{tabular}

